\title{
Fluorescent Dyes with Large Stokes Shifts of 80-200 nm for Optical Microscopy and Nanoscopy
}

\author{
Dissertation \\ for the award of the degree \\ “Doctor rerum naturalium” (Dr. rer. nat.) \\ of the Georg-August-Universität Göttingen \\ within the doctoral program in Chemistry \\ of the Georg-August University School of Science (GAUSS) \\ submitted by

\section{Maksim Sednev} \\ from Nizhny Tagil
}

Göttingen 


\section{Thesis committee}

Prof. Dr. Armin de Meijere, Institute of Organic and Biomolecular Chemistry, GeorgAugust-Universität Göttingen

Prof. Dr. Stefan W. Hell, Department of NanoBiophotonics, Max Planck Instutute for Biophysical Chemistry

\section{Members of the examination board}

Reviewer: Prof. Dr. Armin de Meijere, Institute of Organic and Biomolecular

Chemistry, Georg-August-Universität Göttingen

Second reviewer: Prof. Dr. Stefan W. Hell, Department of NanoBiophotonics, Max

Planck Instutute for Biophysical Chemistry

\section{Further members of the Examination board}

Prof. Dr. Ulf Diederichsen, Institute of Organic and Biomolecular Chemistry, GeorgAugust-Universität Göttingen

Prof. Dr. Claudia Höbartner, Research Group Nucleic Acid Chemistry, Max Planck Instutute for Biophysical Chemistry and Institute of Organic and Biomolecular Chemistry, Georg-August-Universität Göttingen

Prof. Dr. Konrad Koszinowski, Institute of Organic and Biomolecular Chemistry, Georg-August-Universität Göttingen

Prof. Dr. Heinz Neumann, Department of Applied Synthetic Biology, GZMB, GeorgAugust-Universität Göttingen

Date of oral examination: 08.06.2015 


\section{Abstract}

Bright and photostable fluorescent dyes with large Stokes shift are rare, though they are indispensable in optical microscopy, biology and chemistry. The rapid progress in superresolution microscopy based on stimulated emission depletion (STED) phenomenon encouraged us to design and prepare new coumarins and a hybrid carborhodol dye. Variation of electron-withdrawing groups at C-3 and/or C-4 enabled us to create promising coumarin dyes possessing a 3-(2-pyridyl) group $\left(\lambda_{\mathrm{abs}} / \lambda_{\mathrm{em}}=432 / 512 \mathrm{~nm}\right.$ in aqueous phosphate buffer), a 3-(pyrido[1,2-a]pyrrolo[2,1-c]pyrazinium) group (489/587 nm in $\mathrm{MeOH})$ and a fused quinoline ring (453/617 nm in aqueous phosphate buffer). The new dyes were decorated with a polar phosphate group which provided sufficient solubility in aqueous solutions and a carboxylic group which was required for bioconjugation.

The hybrid carborhodol dye was obtained by a combination of (carbo)fluorescein and carbopyronine fluorophores. Due to the broad absorption and emission spectra of the carborhodol in the conjugated form (586/613 nm in aqueous phosphate buffer), the effective Stokes shift is larger in comparison with small Stokes shifts of the parent dyes. This allowed the use of carborhodol in two-color imaging schemes as well as in STED microscopy with a $775 \mathrm{~nm}$ depletion laser. 


\section{Table of contents}

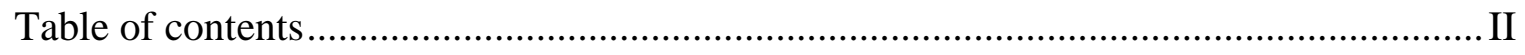

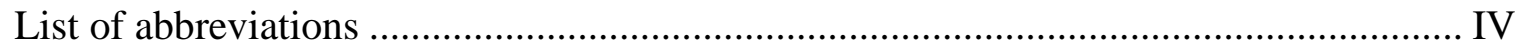



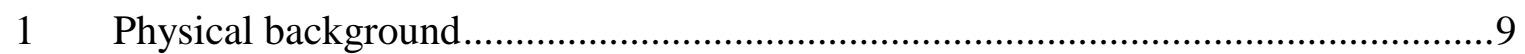

1.1 Main principles of superresolution fluorescence microscopy .....................9

$1.2 \quad$ Large Stokes shift fluorophores .........................................................16

1.2.1 Fluorophores with a Stokes shift provided by photophysical processes .....................................................................................16

1.2.2 Fluorophores with a Stokes shift provided by photochemical

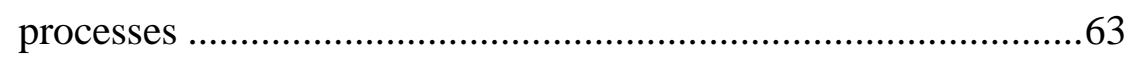

1.2.3 Multifluorophore constructs with pseudo-large Stokes shifts......67

1.3 Applications of large Stokes shift dyes in fluorescence nanoscopy...........71

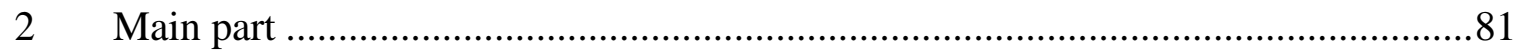

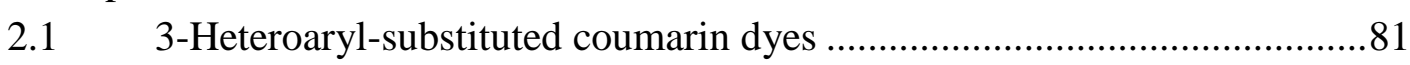

2.1.1 Motivation and key structural elements ....................................81

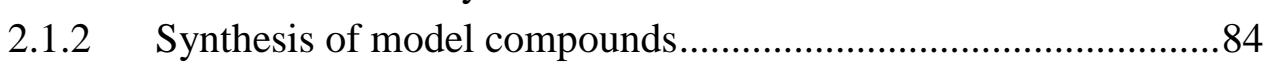

2.1.3 Spectral properties of model coumarin dyes ...............................87

2.1.4 Synthesis of water-soluble coumarins with a phosphate group ...89

2.1.5 Spectral properties and imaging performance of coumarin 308 ..90

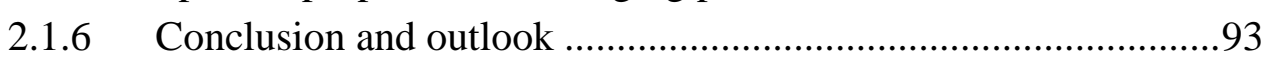

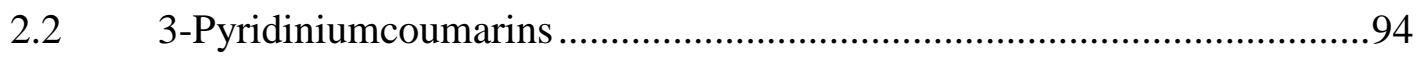

2.2.1 Motivation and key structural elements ....................................94

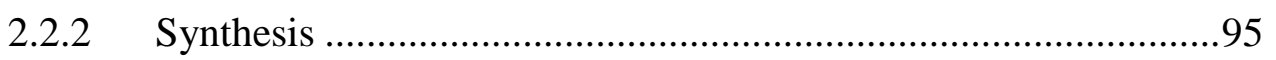

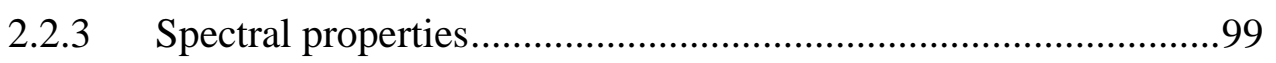

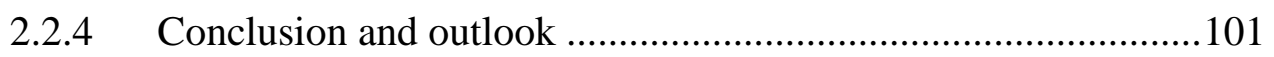

2.3 Synthesis of pyrido- and isoquinolino-fused coumarin dyes ....................102

2.3.1 Motivation and key structural elements ...................................102

2.3.2 Synthesis of model hydrophilic compounds .............................103

2.3.3 Spectral properties of pyrido- and (iso)quinolinocoumarins .....110

2.3.4 Synthesis of water-soluble quinolinocoumarin $\mathbf{3 7 8}$ and its spectral

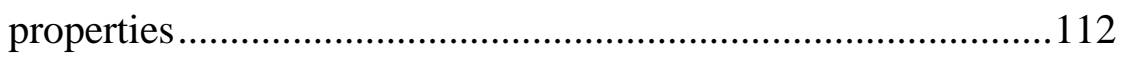

2.3.5 Conclusion and outlook .........................................................113

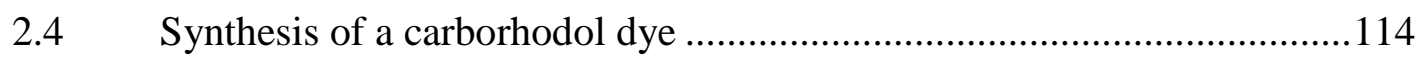

2.4.1 Motivation and key structural elements ...................................114

2.4.2 Synthesis and chemical properties of carborhodol dyes ............116

2.4.3 Properties and imaging performance of carborhodol dyes ........123 
2.4.4 Conclusion and outlook .......................................................130

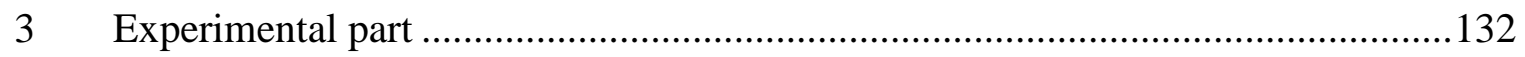

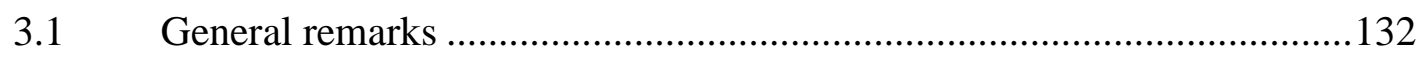

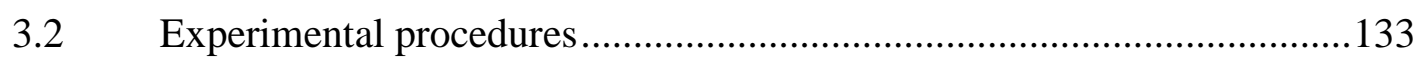

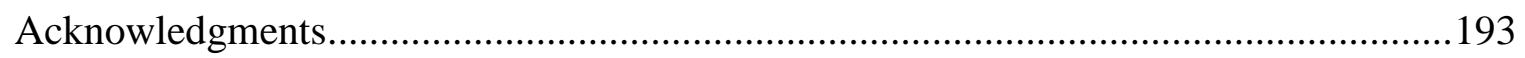

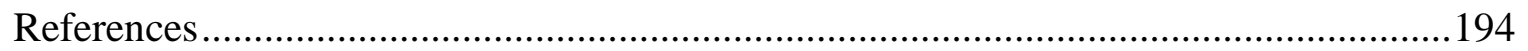

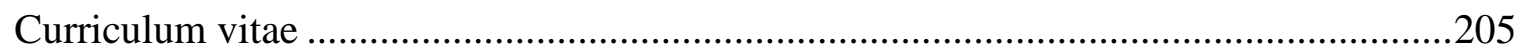




\section{List of abbreviations}

2C STED two-color STED

4-MU 4-methylumbelliferone

AM1 Austin Model 1

APTS 8-aminopyrene-1,3,6-trisulfonic acid

BC benzylcytosine

BG benzylguanidine

BODIPY boron-dipyrromethene

BOPIM boron 2-(2-pyridyl)imidazole

Cbz carboxybenzyl

CT charge transfer

DCC $\quad N, N$-dicyclohexylcarbodiimide

DFT density functional theory

DIEA diisopropylethylamine

DMABN 4- $N, N$-dimethylaminobenzonitrile

DMAP 4- $N, N$-dimethylaminopyridine

DMF $\quad N, N$-dimethylformamide

DMSO dimethyl sulfoxide

DOL degree of labeling

dppf 1,1'-bis(diphenylphosphino)ferrocene

DRET dark resonance energy transfer

dSTORM direct STORM

EDC 1-ethyl-3-(3-dimethylaminopropyl)carbodiimide

ESIPT excited-state intramolecular proton transfer

(F)PALM (fluorescence) photoactivation localization microscopy

Fmoc fluorenylmethoxycarbonyl 
FRET fluorescence resonance energy transfer

GSD ground state depletion

GSDIM GSD followed by individual molecule return

HATU O-(7-azabenzotriazol-1-yl)- $N, N, N$ ', $N$ '-tetramethyluronium hexafluorophosphate

HOMO highest occupied molecular orbital

HPLC high performance liquid chromatography

IC internal conversion

ICT intramolecular CT

IR infrared

ISC intersystem crossing

LDA lithium diisopropylamide

MAO monoamine oxidase

mCPBA m-chloroperbenzoic acid

NHS $\quad N$-hydroxysuccinimidyl

NIR near-IR

ODF oligodeoxyfluoroside

PA-FP photoactivatable fluorescent protein

PBS phosphate buffered saline

pcFRET photochromic FRET

PET photoinduced electron transfer

PPA polyphosphoric acid

PTCA perylene-3,4,9,10-tetracarboxylic acid

PVA polyvinyl acetate

QY quantum yield

RESOLFT reversible saturable optical fluorescent transitions

RFI relative fluorescence intensity

SMLM single-molecule localization methods 


\begin{tabular}{ll} 
SNAFL & seminaphthofluorescein \\
SNAFR & seminaphthorhodafluor \\
SNARF & seminaphthofluorone \\
SNP & single nucleotide polymorphism \\
SRS & stimulated Raman-scattering source \\
STED & stimulated emission depletion \\
STORM & stochastic optical reconstruction microscopy \\
TBAF & tert-butylammonium fluoride \\
TBDMS & tert-butyldimethylsilyl \\
TBET & through-bond energy transfer \\
TFA & trifluoroacetic acid \\
THF & tetrahydrofuran \\
THP & 2-tetrahydropyranyl \\
TICT & twisted ICT \\
UV & ultraviolet \\
\hline
\end{tabular}




\section{Introduction}

Fluorescence microscopy has become an essential tool in modern life sciences. A vast majority ( $80 \%$ ) of all microscopy investigations is still performed with conventional lenses and visible light, despite the significant achievements made by electron and X-ray microscopies. ${ }^{[1]}$ Biological tissues are transparent to visible light to a great extent. This inherent property gives a unique advantage to fluorescent microscopy over other methods and provides the possibility of non-invasive imaging of the interior parts of cells in three dimensions. Furthermore, various cellular constituents, such as proteins, nucleic acids or lipids, can be detected specifically when fluorescence tagging is employed.

However, a fundamental physical barrier known as the Abbe diffraction limit restricts the resolution of conventional fluorescence microscopy (in the visible range of 400-800 nm) to about $200 \mathrm{~nm}$ in the focal plane. Hence, submicron scale cell structures (for example, cristae of a mitochondrion) cannot be resolved. Luckily, as a result of recent innovations, several new super-resolution techniques that fundamentally overcome the diffraction barrier have been developed. For their pioneering work in improving the resolution of fluorescent microscopes, Stefan W. Hell, William E. Moerner and Eric Betzig were awarded with Nobel Prize in Chemistry 2014.

To obtain better results, these new microscopic methods often require more photostable fluorophores with higher binding specifity, greater brightness and an ability to switch between bright and dark states. Moreover, for multicolor imaging experiments, the spectral separation of different labels into two or more excitation or detection channels is required. In this regard, fluorophores with large Stokes shifts are particularly valuable, because they allow reducing the number of detection channels, avoiding cross-talk and simplifying the imaging scheme of a multicolor experiment. Unfortunately, photostable and bright fluorescent dyes with large Stokes shift are still rare and only a few of them are commercially available. Many suppliers offer fluorescent dyes and their conjugates with large Stokes shifts, but do not disclose their chemical structures and important photophysical properties. The lack of these data complicates the proper choice of the fluorescent dye for a particular imaging application. Furthermore, this also hampers the progress in basic research aimed at the creation of new fluorophores. 
This work deals with the design and synthesis of new fluorophores with large Stokes shifts. The dissertation consists of two parts. The first part reviews the basic principles of super-resolution microscopy, the published data on large Stokes shift dyes and their use in super-resolution microscopy. The second part describes our own results and is further divided into four sections. In the first three sections, the synthesis of new coumarin-based dyes, their photophysical properties and application in super-resolution microscopy are described. The last section introduces new dyes - carborhodols, which represent asymmetric hybrids of fluorescein (or carbofluorescein) and carbopyronines. Owing to broader absorption and emission bands exhibited by carborhodols in comparison with parent carbopyronines or (carbo)fluoresceins, it was successfully used in two-color imaging as a large Stokes shift dye. 


\section{Physical background}

\subsection{Main principles of superresolution fluorescence microscopy}

The processes associated with light absorption by a molecule can be conveniently illustrated by a Perrin-Jablonski diagram (see Scheme 1). It displays the relative positions of molecular energy levels and possible photophysical processes which follow the act of photon absorption. Thus, upon excitation, a molecule in the singlet ground state $\mathrm{S}_{0}$ gains energy and enters one of the vibrationally excited levels of the singlet excited state $S_{1}$. Since the energy gap between various vibrational levels is relatively small (in comparison to the gap between electronic states), the molecule undergoes a very fast $\left(10^{-12}-10^{-10} \mathrm{~s}\right)$ process of vibrational relaxation to the lowest vibrational level of $\mathrm{S}_{1}$. Afterwards, a few deactivation processes can take place. One of them is fluorescence, a radiative transition from the $S_{1}$ to one of the vibrationally excited levels of the ground state $S_{0}$. After the very fast vibrational relaxation, the molecule occupies the ground state $\mathrm{S}_{0}$. The energy of the absorbed photon is higher than that of the emitted photon, and this explains the origin of the Stokes shift which is determined as a separation between the maxima of absorption and emission bands (usually measured in $\mathrm{nm}$ or $\mathrm{cm}^{-1}$ ).

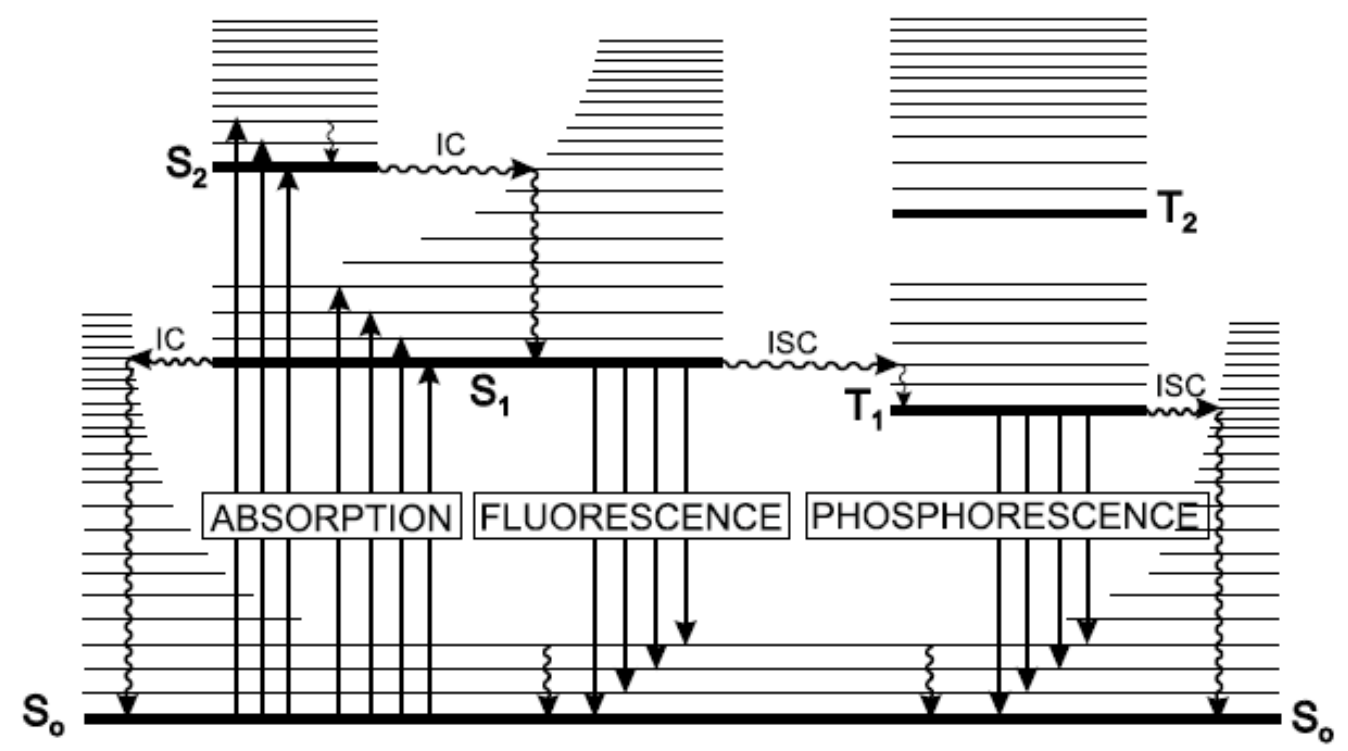

Scheme 1 Jablonski diagram: transitions between the ground and excited states and illustrating the origin and positions of absorption, emission, and phosphorescence bands. ${ }^{[2]}$ 
Other deactivation processes are represented by internal conversion (IC) and intersystem crossing (ISC). Internal conversion is a nonradiative transition between two electronic states of the same spin multiplicity. This process can compete with other deactivation processes in solution where the excess energy can be transferred to the solvent through collisions of the excited molecule with the surrounding solvent molecules. Intersystem crossing is a nonradiative transition between two isoenergetic vibrational levels belonging to electronic states of different multiplicities. Formally, this process is forbidden and occurs slowly, unless there is a significant spin-orbit coupling. The presence of heavy atoms, such as $\mathrm{Br}$, Se, I, Pb etc. increases spin-orbit coupling and therefore favors intersystem crossing. All deactivation processes reduce the probability of radiative $\mathrm{S}_{1} \rightarrow \mathrm{S}_{0}$ transition and thus diminish the fluorescence QY. To obtain a large signal-to-noise ratio in fluorescence microscopy, fluorophores with large brightness (a product of extinction coefficient $\varepsilon$ and fluorescence quantum yield $\Phi_{\mathrm{fl}}$ ) must be employed.

To obtain an image, the conventional confocal microscope - a standard tool in modern life sciences - rapidly scans a sample with a focused beam of light and collects emitted photons from all fluorescent species excited by this beam. According to the Abbe principle, light with wavelength $\lambda$, travelling in a medium with refractive index and converging with angle $\theta$ will make a spot of size $d$, described by Eq. 1 .

$$
d=\frac{\lambda}{2 n \sin \theta}
$$

Objective lenses of modern microscopes have a semiaperture angle $\theta$ close to $67^{\circ}$, whereas the refractive index of common working media ranges from $n=1.00$ (for air) to 1.52 (for immersion oil). If we illuminate the sample with green light having a wavelength of $500 \mathrm{~nm}$, it will be impossible to focus light onto a spot smaller than $164 \mathrm{~nm}$ and resolve structures smaller than this spot. However, in many cases, a better optical resolution is desirable. For example, an adequate imaging and resolving of all parts of a nuclear pore complex of protein clusters is an extremely challenging task for fluorescence microscopy because of its small size (145 nm in diameter and $80 \mathrm{~nm}$ length $\left.{ }^{[3]}\right)$ which is below the diffraction limit. Although electron microscopy provides better resolution, it is incompatible with live specimens and requires tedious preparation and fixation techniques, which can be destructive towards the features of interest. 
Optical resolution of the light microscope considered to be limited by the diffraction limit for more than a century. However, after several super-resolution techniques have been established, the diffraction barrier was broken. The first of these techniques is stimulated emission depletion microscopy (STED). ${ }^{[4]}$ In the method of STED, a focused laser pulse excites fluorescence in a spot of a diffraction limited size (see Figure 1), and immediately after the excitation a red-shifted (to prevent re-excitation) doughnut-shaped STED beam is applied. It features zero intensity only at the very center and depletes the fluorescence of excited fluorophores on the periphery of the spot by stimulated emission $\left(S_{1} \rightarrow S_{0}\right)$.

a

(A)

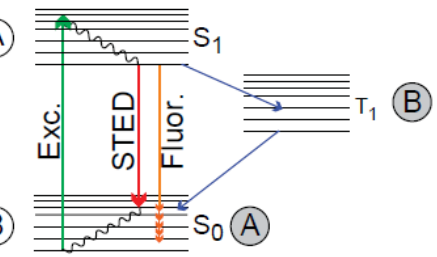

b

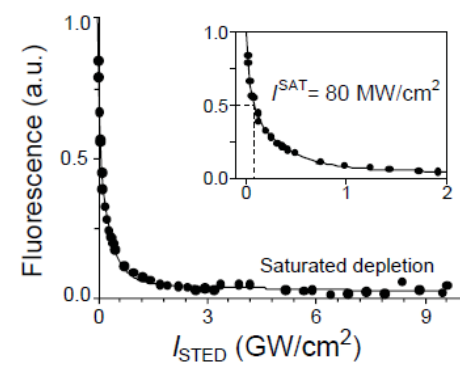

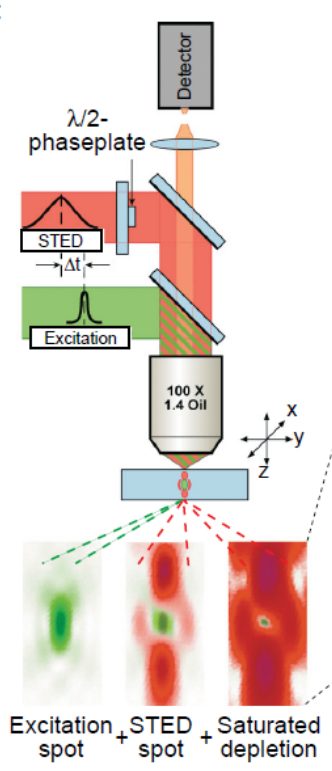

d

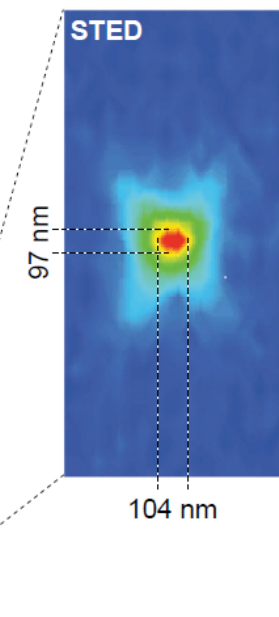

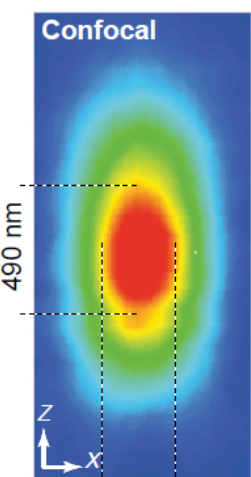

$244 \mathrm{~nm}$

Figure 1 Physical conditions, setup and typical focal spot for STED: ${ }^{[5]}$ a) Energy diagram of an organic fluorophore. b) Saturated depletion of the excited state $S_{1}$ with increasing STED pulse intensity $I_{\text {STED }}$. c) Sketch of a point-scanning STED microscope. d) Fluorescent spot in the STED and in the confocal microscope.

In order to "squeeze" the central spot, the depletion rate should exceed the rate of spontaneous transition to the ground state $S_{0}$. Typical values of fluorescence lifetimes for organic fluorophores $\left(\tau_{\mathrm{fl}} \approx 10^{-9} \mathrm{~s}\right)$ and optical cross-sections for their $\mathrm{S}_{0} \rightarrow \mathrm{S}_{1}$ transitions $\left(\sigma \approx 10^{-16} \mathrm{~cm}^{2}\right)$ imply that intensity of STED pulse $I_{\mathrm{STED}}>I_{\mathrm{S}}=\left(\sigma \tau_{\mathrm{fl}}\right)^{-1} \approx 10 \mathrm{MW} / \mathrm{cm}^{2}$, where $I_{\mathrm{S}}$ is the effective saturation intensity which can be defined as the intensity at which probability of fluorescence is reduced by half. The optical resolution in STED is defined by Eq. 2. 


$$
d \approx \frac{\lambda}{2 n \sin \theta \sqrt{1+I_{S T E D} / I_{S}}}=\frac{d_{C}}{\sqrt{1+I_{S T E D} / I_{S}}}
$$

The value $d_{C}$ represents the resolution of a diffraction-limited system. According to Eq. 2, to obtain a significant improvement in resolution, very high pulse intensities $I_{\text {STED }}$ should be applied. Such enormous light intensities $\left(>100 \mathrm{MW} / \mathrm{cm}^{2}\right)$ inevitably cause photobleaching of fluorophores, and therefore only highly photostable dyes are suitable for STED microscopy. In addition, to increase sensitivity and accordingly reduce imaging time, fluorophores with high fluorescence quantum yields are required.

Let us briefly consider the principles of another superresolution method - ground state depletion microscopy (GSD). ${ }^{[6]}$ In this method the triplet state $T_{1}$ of a fluorophore is used as a dark "off”-state B (see Figure 1a), instead of the ground state $\mathrm{S}_{0}$ used in STED. Since lifetime of the triplet state $T_{1}$ is much longer than that of the singlet state $S_{1}$, light intensities required for a saturable conversion of fluorophores to the triplet state $\mathrm{T}_{1}$ are considerably smaller $\left(<10 \mathrm{KW} / \mathrm{cm}^{2}\right)$. However, not all molecules can be necessarily transferred to "dark" (triplet) states, and the fluorophores in the triplet (biradical) state, due to their long lifetimes and reactive nature of biradicals, can easily undergo various photochemical reactions which eventually lead to their bleaching. This problem is especially important in the presence of oxygen. Since the GSD parameters depend strongly on the nature of the fluorescent dyes and the environment, carefully chosen dyes and mounting media (oxygen scavengers) are required. ${ }^{[7]}$ Furthermore, wavelengths leading to excitation from $\mathrm{T}_{1}$ to higher triplet states should be avoided.

The principles of STED and GSD microscopies were unified into the concept of reversible saturable optical fluorescent transitions (RESOLFT). This paradigm is applicable to all methods based on switching between two distinguishable and reversibly switchable, and thermally stable "bright” and “dark” states. Presumably, at least one transition between these states can be optically induced. The RESOLFT concept can be applied not only to transitions between electronic states, but also to various reversible photochemical transformations (isomerizations, cyclizations etc.). For example, in RESOLFT microscopy with photoswitchable fluorescent proteins, ${ }^{[8]}$ illumination with light of appropriate wavelength induces the E-Z isomerization of fluorescent proteins, which change their conformation, thus gaining or losing their ability to emit light. Low light intensities (a few $\mathrm{W} / \mathrm{cm}^{2}$ ) can be already sufficient to provide a full conversion and 
overcome the diffraction barrier. The main disadvantage of reversibly switchable fluorescent proteins is their low fluorescence quantum yield. Therefore, for an acceptable image contrast, it is often necessary to use high protein concentrations. Moreover, formation of dimers and oligomers as well as moderate photostability can sometimes compromise biological imaging. ${ }^{[9]}$

Along with photoswitchable proteins, photochromic organic compounds can also reversibly change their structure upon illumination. However, not many of them are fluorescent. This problem was circumvented in the method of photochromic energy transfer (pcFRET). ${ }^{[10]}$ In this method a combination of a photochromic and a fluorescent compound is used. One of the isomers of a photochromic compound absorbs visible light and is used as an energy acceptor for an excited fluorophore acting as a donor. As a result, the RESOLFT microscopy based on reversible switching of small fluorescent photochromic labels was implemented. ${ }^{[11]}$ Similarly to fluorescent proteins, only small light intensities are needed for efficient switching of photochromic compounds.

In comparison to RESOLFT techniques, where the position of the subdiffraction-sized emitting spot is known and well controlled, single-molecule localization microscopy (SMLM) methods rely on stochastical switching of single fluorophores. Stochastically distributed positions are sparse, contain only a small fraction of fluorophores in the bright state and do not overlap with each other, thus giving an opportunity to precisely localize these fluorophores using appropriate algorithms. It is important that at certain moment the distances between the "activated" (bright) markers are greater than the diffraction-limited distances ( 200 nm), so that these markers can be localized and detected independently (separately). The localization precision ( $\Delta l o c)$ depends on the number of collected protons $N$ as defined by Eq. 3 (where $\Delta$ is the full width at half maximum of the point spread function).

$$
\Delta l o c \approx \frac{\Delta}{\sqrt{N}}
$$

Photoactivatable fluorescent protein (PA-FP) molecules that are initially found in a dark (non-fluorescent) state can be either reversibly or irreversibly activated by irradiation at one wavelength, and then can be visualized by excitation at a second wavelength. In a technique called (fluorescence) photoactivation localization microscopy ((F)PALM), ${ }^{[12]}$ a 
sample with target proteins fused with PA-FPs is continuously excited by pulses of light from a laser at a wavelength close to the excitation maximum of the PA-FPs. The excitation is maintained until a large population of inactivated PA-FPs is obtained by reversible "bleaching”, thus creating sparse fields of individually resolvable single molecules. After recording several image frames (individual images), the "bleaching” process leads to a mean molecular separation larger than that needed for isolation of individual molecules which are still in the active state. At this point, a pulse from another laser at a shorter wavelength capable of reactivating the PA-FPs molecules from the inactive state is applied. The pulse duration and intensity is chosen so that the population of active PAFPs is increased to higher, but still resolvable level. The process of "bleaching”, activation and recording is repeated many times $\left(>10^{4}\right)$, until the whole set of inactivated molecules is used up. The signals from every molecule are summed across all recorded frames and then fitted using a mathematical algorithm giving position coordinates of the molecule and a standard deviation of this position. A final super-resolved image is rendered usually by representing each molecule as a two-dimensional Gaussian with the amplitude proportional to the number of collected photons and the standard deviation which depends on the localization precision. Genetically encoded labeling used in (F)PALM easily circumvents problems caused by unspecific binding. It is also compatible with live-cell imaging. However, fluorescent proteins often exhibit lower photon counts than organic dyes, and therefore, it can be difficult sometimes to obtain the full super-resolved image.

Another SMLM technique, stochastic optical reconstruction microscopy (STORM), ${ }^{[13]}$ is based on a similar fundamental principle as (F)PALM. However, instead of endogenously expressed PA-FPs, STORM relies on immunolabeling of the sample with antibodies tagged with optically switchable organic fluorophores. Originally, a pair of cyanine dyes, Cy3 and Cy5, known as "cyanine switch" was used. Cy3 served here as an "activator" that facilitates the transition of Cy5 to the “on"-state. The imaging procedure is pretty similar to that of (F)PALM: first, a red laser switches nearly all fluorophores to a stable dark state, and then a pulse of a green laser switches a small and random number of fluorophores to the "on”-state, and a frame is taken. This procedure is repeated many times, and at the end of the whole sequence, individual frames are processed and merged into a final image.

The necessity of double labeling of antibodies with activator-reporter pairs poses certain problems. To circumvent these problems, the so-called direct STORM (dSTORM) 
method was proposed. ${ }^{[14]}$ In this technique, a green laser with a power 200 times higher than that in STORM was used. It efficiently turns a small subset of inactivated fluorophores (single dyes) to the bright state. A laser with a higher power made possible to use conventional and commercially available dyes (Cy5 and similar ones) making the experiment and sample preparation much easier than in the original STORM method.

In comparison to (F)PALM, organic fluorophores in (d)STORM allow obtaining a brighter stain and thus, a better image quality. However, as all techniques which rely on labeling with tagged antibodies, (d)STORM is also vulnerable to background noise caused by non-specific binding.

In addition to (F)PALM and STORM another super-resolution method was proposed. In ground state depletion microscopy followed by individual molecule return (GSDIM), ${ }^{[15]}$ stochastic single-molecule “on”-switching is performed without any photochemical transformation and relies only on basic transitions of standard markers. This technique operates using the same mechanism employed in GSD microscopy, i.e. transferring a synthetic dye to its triplet state $\mathrm{T}_{1}$ (or another metastable dark state). But unlike GSD, GSDIM implies that a fluorophore recovers to its ground state $S_{0}$ only once. Images of the emitters are recorded, only when they spontaneously return to the ground state.

For the highest precision in localization, the number of emitted photons per switching cycle should be maximized (see Eq. 3). The relative brightness is proportional to the product of the molar extinction coefficient $\varepsilon$ and the fluorescence quantum yield $\Phi_{f l}$ of the fluorophore. For (F)PALM and STORM, fluorescent probes should also exhibit high switching reliability, high efficiency of transition to the "dark" state and a low fatigue rate. Furthermore, the reversible photobleaching and photoactivation rates should be balanced in a way that only a small fraction of fluorophores is activated at any particular time.

The image acquisition time in SMLM methods is defined by the number of the determined molecular positions needed for the reconstruction of the final image. Due to the intrinsic stochastic nature of all these methods it will be always not clear whether all positions of molecules have been recorded or not. This issue (when the image acquisition has to be stopped) is particularly important in live-cell imaging where the recording speed is crucial. In contrast to SMLM, in RESOLFT-type microscopy (including STED) the speed of the scanning process defines the acquisition time. The use of fast beam scanners 
allowed recording of a $4.5 \mu \mathrm{m}^{2}$ field of view with a focal spot size of $62 \mathrm{~nm}$ at video rates (STED with 28 frames per second). ${ }^{[16]}$ Moreover, there is a great potential in parallelization of the scanning procedure. In a recent report, ${ }^{[17]}$ it has been shown that RESOLFT microscopy can be effectively parallelized using two incoherently superimposed orthogonal standing light waves. The intensity minima of the resulting pattern act as more than 100000 “doughnuts”. As a result, superresolution images of living cells in $12000 \mu \mathrm{m}^{2}$ fields of view can be recorded in less than 1s using fluorescent proteins with a relatively slow switching kinetics.

One of the main advantages of fluorescent microscopy is the ability to use several fluorescent labels to target different specimens and produce multicolor images that help to identify many structural features of biological objects and interactions between them. Twocolor STED (2C STED) using two separate sets of excitation and STED wavelengths for spectrally separated dyes has been reported. ${ }^{[18]}$ However, this approach is technically demanding. Another approach ${ }^{[19]}$ uses a standard fluorophore with a small Stokes shift $(10-30 \mathrm{~nm})$ in combination with a large Stokes shift (usually more than $80 \mathrm{~nm}$ ) dye which have (partially) overlapping emission spectra. Two excitation wavelengths for two dyes are used to distinguish them. At the same time, due to similar emission spectra, only one STED beam can be used.

The common feature of many fluorescent dyes with large Stokes shifts is their relatively low brightness and poor photostability. These drawbacks limit their wide use in modern methods of optical microscopy, especially in STED nanoscopy. The present work deals with the design and synthesis of new bright and photostable fluorophores with large Stokes shift. In the following sections, common photophysical mechanisms providing large Stokes shifts as well as common classes of large Stokes shift dyes and their use in the imaging applications will be discussed.

\subsection{Large Stokes shift fluorophores}

\subsubsection{Fluorophores with a Stokes shift provided by photophysical processes}


Upon excitation of a molecule, movements of electrons occur so fast (in ca. $10^{-15} \mathrm{~s}$ ), that the atom nuclei in the molecule remain nearly stationary. In other words, the molecular geometry does not change in the course of absorption of a photon (Franck-Condon principle). However, the newly formed vibronic state is unstable, and the molecule quickly relaxes (in ca. $10^{-13}$ ) to its equilibrium vibronic state. During this process, a part of the absorbed energy turns into heat. Similar transitions occur during emission. After a "fluorescence" photon is emitted, the molecule is found in the electronic state $\mathrm{S}_{0}$ which retains the geometry of the excited state $S_{1}$. After vibrational relaxation, a part of the energy of the absorbed photon is again converted into heat. Thus, the initially absorbed energy of a photon is partially converted to heat in the course of absorption and emission processes. In most cases, the dipole moment of a fluorophore in the excited state differs from that in the ground state. Therefore, after excitation, the solvent molecules that surround a molecule of the fluorophore undergo relaxation, leading to a relaxed excited state of lower energy. With increasing solvent polarity, the energy of the relaxed state becomes lower. As a result, the emission spectrum exhibits a red-shift. Geometrical relaxation and relaxation of the solvent media are two photophysical processes responsible for the generation of the Stokes shift. Therefore, in order to increase a Stokes shift, one should design molecules with large differences between equilibrated geometries and dipole moments in the ground and excited states.

Unfortunately, these differences are difficult to predict, and the design of new fluorophores still remains mostly a matter of trial and error. Despite the significant advances in quantum-mechanical methods, it is still impossible to predict accurately all important properties of fluorescent dyes, such as band shapes and maxima of absorption and emission spectra in different solvents, Stokes shifts, molar extinction coefficients and fluorescence quantum yields. Therefore, when designing a new fluorophore, synthetic chemists often rely on the known data for similar dyes and general empirical guidelines. For example, in the case of fluorophores exhibiting intramolecular charge transfer (ICT) upon excitation, stronger or weaker acceptors and/or donors, or new $\pi$-systems can be introduced to the core fluorophore in order to "tune" the "push-pull" effect or expand the conjugation system of the fluorophore, thus providing bathochromic and bathofluoric shifts and increasing the molar extinction coefficient. However, regularities concerning the positions of these substituents at the dye scaffold are still unclear. A useful report 
generalizing the structure-property relationships and providing detailed guidelines for the design of certain classes of fluorophores has been published recently. ${ }^{[20]}$

There is no general theory that could explain structure-property relationships, and it is difficult to design and prepare a new fluorophore with required properties. Only a few fluorophore classes, such as cyanine dyes, coumarins, rhodamines (xanthene dyes), carbopyronines, BODIPYs, Si-rhodamines and their hybrids have been widely used in life sciences. Among fluorescent dyes with large Stokes shift, coumarins (and hybrid dyes based on coumarins) are very important. Almost all commercially available fluorophores with large Stokes shifts with $\lambda_{\text {exc }} \geq 400 \mathrm{~nm}$ contain a coumarin fragment (see Table 1). A few notable exceptions are represented by Lucifer Yellow and Atto dyes, 430LS and 490LS. Other dyes with large Stokes shifts include benzooxazole or triphenylpyrazoline derivatives. Although these dyes have good fluorescence quantum yields, they require UV light for excitation, which is often incompatible with imaging of biological samples.

Table 1 Selected commercially available dyes with large Stokes shift and with $\lambda_{\mathrm{abs,max}}>390 \mathrm{~nm}$.

\begin{tabular}{|c|c|c|c|c|c|c|c|}
\hline Name & Structure & $\begin{array}{c}\lambda_{\text {abs,max }}, \\
n m\end{array}$ & $\begin{array}{c}\lambda_{\mathrm{em}, \max } \\
\mathrm{nm}\end{array}$ & $\begin{array}{c}\varepsilon, \\
\mathrm{M}^{-1} \mathrm{~cm}^{-1}\end{array}$ & $\Phi_{\mathrm{fl}}$ & $\begin{array}{l}\tau_{\mathrm{fl}} \\
\mathrm{ns}\end{array}$ & solvent \\
\hline $\begin{array}{l}\text { Abberior } \\
\text { STAR } \\
\text { 440SXP }\end{array}$ & & 436 & 515 & 22700 & 0.68 & 3.3 & PBS \\
\hline $\begin{array}{l}\text { Abberior } \\
\text { STAR } \\
\text { 470SXP }\end{array}$ & & 472 & 624 & 29000 & 0.12 & 0.8 & PBS \\
\hline $\begin{array}{l}\text { Abberior } \\
\text { STAR } \\
520 \mathrm{SXP}\end{array}$ & & 522 & 632 & 42500 & 0.15 & - & PBS \\
\hline $\begin{array}{c}\text { Alexa } \\
\text { Fluor } 430\end{array}$ & & 431 & 541 & 16000 & 0.55 & - & $\mathrm{H}_{2} \mathrm{O}$ \\
\hline
\end{tabular}




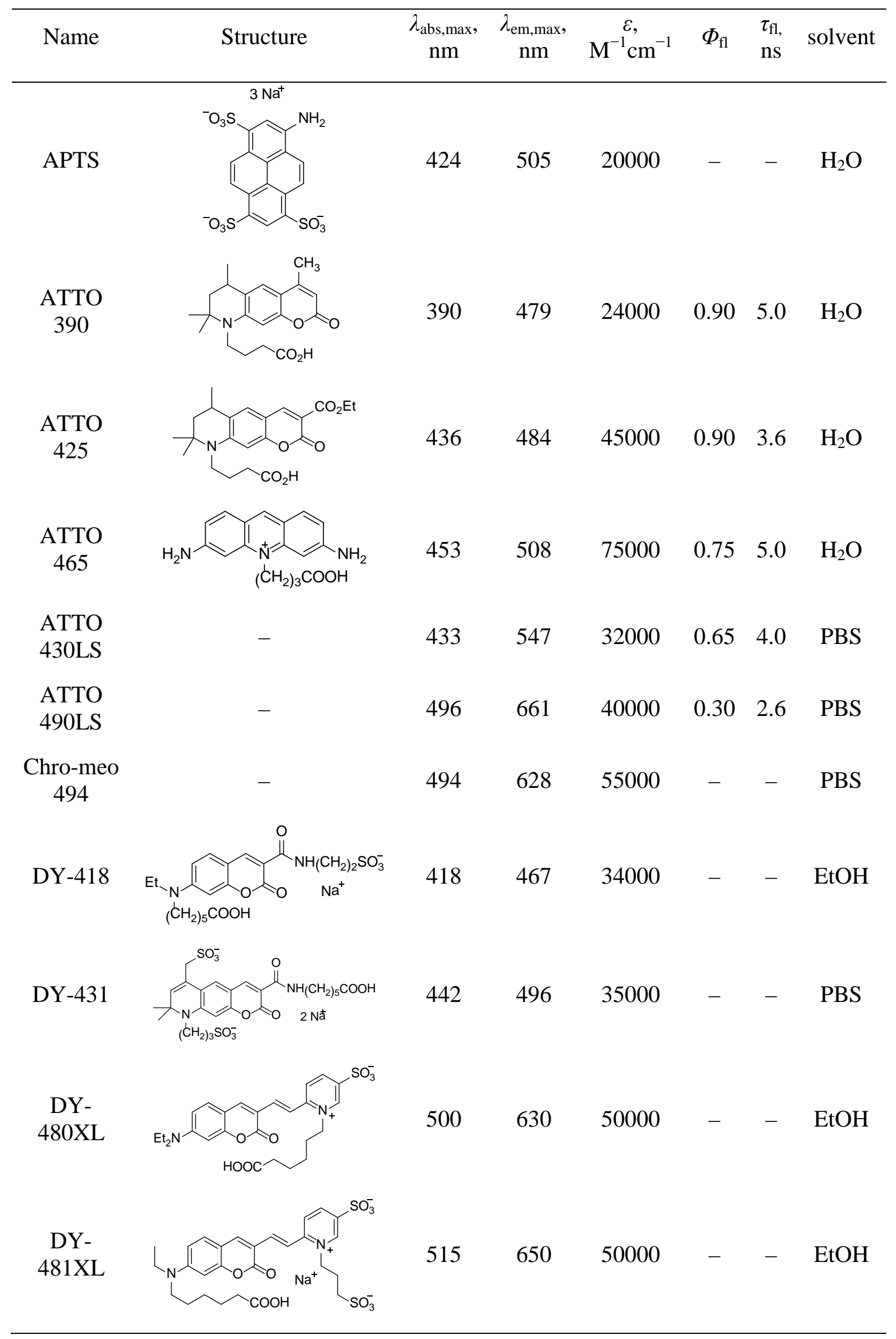




\begin{tabular}{|c|c|c|c|c|c|c|c|}
\hline Name & Structure & $\begin{array}{c}\lambda_{\text {abs,max }}, \\
\text { nm }\end{array}$ & $\begin{array}{c}\lambda_{\text {em,max }}, \\
n m\end{array}$ & $\mathrm{M}^{-1} \mathrm{~cm}^{-1}$ & $\Phi_{\mathrm{fl}}$ & $\begin{array}{l}\tau_{\mathrm{fl}}, \\
\mathrm{ns}\end{array}$ & solvent \\
\hline $\begin{array}{c}\text { DY- } \\
485 X L\end{array}$ & & 482 & 560 & 48000 & - & - & $\mathrm{H}_{2} \mathrm{O}$ \\
\hline $\begin{array}{c}\text { DY- } \\
\text { 510XL }\end{array}$ & & 493 & 585 & 40000 & - & - & $\mathrm{H}_{2} \mathrm{O}$ \\
\hline $\begin{array}{c}\text { DY- } \\
\text { 511XL }\end{array}$ & & 510 & 595 & 47000 & - & - & $\mathrm{EtOH}$ \\
\hline $\begin{array}{c}\text { DY- } \\
520 X L\end{array}$ & & 520 & 664 & 50000 & - & 2.1 & $\mathrm{EtOH}$ \\
\hline $\begin{array}{c}\text { DY- } \\
\text { 521XL }\end{array}$ & & 523 & 668 & 50000 & - & - & $\mathrm{EtOH}$ \\
\hline $\begin{array}{c}\text { DY- } \\
601 X L\end{array}$ & & 606 & 663 & 85000 & - & - & $\mathrm{EtOH}$ \\
\hline $\begin{array}{c}\text { DyLight } \\
\text { 485-LS }\end{array}$ & Coumarin & 485 & 559 & 50000 & - & - & $\mathrm{EtOH}$ \\
\hline $\begin{array}{c}\text { DyLight } \\
\text { 510-LS }\end{array}$ & Co & 509 & 590 & 50000 & - & - & $\mathrm{EtOH}$ \\
\hline $\begin{array}{l}\text { DyLight } \\
\text { 515-LS }\end{array}$ & Coumarin & 519 & 648 & 50000 & - & - & $\mathrm{EtOH}$ \\
\hline $\begin{array}{l}\text { DyLight } \\
\text { 521-LS }\end{array}$ & Coumarin & 526 & 666 & 50000 & - & - & $\mathrm{EtOH}$ \\
\hline $\begin{array}{l}\text { Krome } \\
\text { Orange }\end{array}$ & - & 398 & 528 & 17665 & - & - & $\mathrm{H}_{2} \mathrm{O}$ \\
\hline $\begin{array}{l}\text { Lucifer } \\
\text { Yellow }\end{array}$ & & 428 & 540 & 11500 & 0.21 & - & $\mathrm{H}_{2} \mathrm{O}$ \\
\hline
\end{tabular}




\begin{tabular}{|c|c|c|c|c|c|c|c|}
\hline Name & Structure & $\begin{array}{c}\lambda_{\text {abs,max }}, \\
\text { nm }\end{array}$ & $\begin{array}{c}\lambda_{\text {em,max }}, \\
n m\end{array}$ & $\mathrm{M}^{-1} \mathrm{~cm}^{-1}$ & $\Phi_{\mathrm{fl}}$ & $\begin{array}{l}\tau_{\mathrm{fl}}, \\
\mathrm{ns}\end{array}$ & solvent \\
\hline Nile Red & & 552 & 636 & 43000 & - & - & $\mathrm{MeOH}$ \\
\hline $\begin{array}{l}\text { Pacific } \\
\text { Blue }\end{array}$ & & 400 & 447 & 29500 & 0.75 & - & PBS \\
\hline $\begin{array}{l}\text { Pacific } \\
\text { Green }\end{array}$ & - & 410 & 500 & - & - & - & $\mathrm{H}_{2} \mathrm{O}$ \\
\hline $\begin{array}{l}\text { Pacific } \\
\text { Orange }\end{array}$ & - & 400 & 551 & - & - & - & $\mathrm{H}_{2} \mathrm{O}$ \\
\hline V500 & - & 415 & 500 & - & - & - & - \\
\hline
\end{tabular}

Coumarin itself (Figure 2) shows no fluorescence at room temperature and has only a weak absorption in the near UV region, but if C-6 or/and C-7 is substituted with an electron-donor group (such as hydroxyl or amino group), an intense blue-green emission appears. It originates due to a "push-pull” effect between the electron-donor group(s) at C-6(7) and the electron-withdrawing lactone moiety. 7-Hydroxy- and 7-aminocoumarins have a long history as laser dyes. ${ }^{[21]}$<smiles></smiles>

Figure 2 Coumarin (2H-chromen-2-one) and its atom numbering.

4-Methyl-7-hydroxycoumarin or 4-methylumbelliferone (4-MU) has the most red-shifted absorption band with a maximum at $360 \mathrm{~nm}\left(\varepsilon=17000 \mathrm{M}^{-1} \mathrm{~cm}^{-1}\right.$ in aqueous phosphate buffer at $\mathrm{pH} 10)$ and emits blue light with a maximum centered at $450 \mathrm{~nm}\left(\Phi_{\mathrm{fl}}=0.63\right.$, Stokes shift of $90 \mathrm{~nm}) .{ }^{[22]}$ Due to presence of the ionizable hydroxyl group with $\left(\mathrm{pK}_{\mathrm{a}}=\right.$ 7.8), the spectra of 4-MU are sensitive to $\mathrm{pH}$ changes. Fluorination of the 4-methylumbelliferone scaffold has minor effects on the absorption and fluorescence spectra, but fluorinated derivatives have higher fluorescence QYs and better photostability. In contrast, 7-hydroxy-4-trifluoromethylcoumarins have significantly lower QYs 
than the parent compound. The hydroxyl group is slightly acidic, and it is ionized in basic solution. Therefore such compounds are soluble in water. However, if 7-hydroxycoumarins contain electron acceptor groups at C-3 or C-4, the fluorescence of their solutions rapidly fades out (due to decomposition). ${ }^{[21 b]} 7$-Hydroxycoumarins are emissive only in their anionic forms, and this makes them unattractive for applications at physiological $\mathrm{pH}$ values: these compounds will be neutral and, therefore, nonfluorescent. A great improvement is 6,8-difluoro-7-hydroxycoumarin (Pacific Blue ${ }^{[22]}$ ) with a reduced $\mathrm{pK}_{\mathrm{a}}$ of 3.7. The greater acidity makes this dye predominantly anionic at physiological $\mathrm{pH}$. However, the anionic nature of this fluorophore is undesirable in some cases. ${ }^{[23]}$

Introduction of acceptor aroyl groups at C-3 of 7-hydroxy- or 7-aminocoumarins provides bathochromic and bathofluoric shifts, but, at the same time, significantly increases the rate of ISC. As a result, their fluorescent QYs decrease. These compounds can be used as efficient triplet sensitizers. ${ }^{[24]}$

A “red" spectral shift and a higher acidity of 7-hydroxycoumarins were achieved by introducing electron-withdrawing groups at C-3 of the coumarin ring. ${ }^{[25]}$ Table 2 contains the spectral data for 7-hydroxycoumarins 1-9 and 4-cyano-7-hydroxycoumarins 10-14 with various substituents at C-3 (Figure 3). In aqueous borate buffer ( $\mathrm{pH} 9$ ), the spectra of compounds 1-7, which have a heterocyclic residue at C-3 and an unsubstituted position 4, have an intense absorption band in the 405-439 $\mathrm{nm}$ range and an emission maximum between 470 and $500 \mathrm{~nm}$. Compounds 8 and 9 with carboxylic and phenyl substituents at C-3 absorb and emit at shorter wavelengths $\left(\lambda_{\text {abs,max }} / \lambda_{\text {em,max }}=386 / 448 \mathrm{~nm}\right.$ and $383 / 462$ $\mathrm{nm}$, respectively). Particularly remarkable is 7-hydroxy-2-thienylcoumarin $\mathbf{7}$ which has the most red-shifted fluorescence maximum (500 nm) and the largest Stokes shift (93 nm) in this group. The introduction of a 4-cyano group at C-4 results in considerable bathochromic and bathofluoric shifts (compared with the corresponding 4-unsubstituted analogs). All 3-substituted 4-cyano-7-hydroxycoumarins (compounds 10-14) have absorption maxima in the range between 487 and $505 \mathrm{~nm}$ and exhibit bright yellow-orange fluorescence in basic aqueous solutions (except compound 14 with a 2-benzimidazolyl substituent which showed weak fluorescence). Interestingly, the benzazole-substituted 4cyano-7-hydroxycoumarins retain large Stokes shifts typical for coumarin dyes. Closely related compounds were used as polarity-sensitive indicators of biochemical processes. ${ }^{[26]}$ 


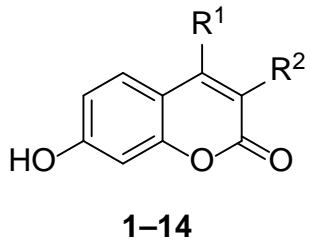

Figure 3 Substituted 7-hydroxycoumarins 1-14.

Table 2 Spectral data of 7-hydroxycoumarins 1-9 and 4-cyano-7-hydroxycoumarins 10-14 with various substituents at $\mathrm{C}-3$ in aqueous borate buffer at $\mathrm{pH} 9$.

\begin{tabular}{|c|c|c|c|c|c|c|}
\hline Compound & $\mathrm{R}^{1}$ & $\mathrm{R}^{2}$ & $\begin{array}{c}\lambda_{\mathrm{abs}, \max }, \\
\mathrm{nm}\end{array}$ & $\begin{array}{c}\varepsilon, \\
\mathrm{M}^{-1} \mathrm{~cm}^{-1}\end{array}$ & $\begin{array}{c}\lambda_{\mathrm{em}, \max }, \\
\mathrm{nm}\end{array}$ & $\begin{array}{c}\Delta \lambda^{\mathrm{a}}, \\
\mathrm{nm}\end{array}$ \\
\hline 1 & $\mathrm{H}$ & 2-benzoxazolyl & 427 & 44300 & 471 & 44 \\
\hline 2 & $\mathrm{H}$ & 2-benzothiazolyl & 439 & 47000 & 490 & 51 \\
\hline 3 & $\mathrm{H}$ & $\begin{array}{c}\text { 5-methyl-7-sulfonato-2- } \\
\text { benzoxazolyl }\end{array}$ & 431 & 44000 & 470 & 39 \\
\hline 4 & $\mathrm{H}$ & 5-chloro-2-benzoxazolyl & 425 & $30600^{\mathrm{b}}$ & 472 & 47 \\
\hline 5 & $\mathrm{H}$ & 2-benzimidazolyl & 427 & $33000^{\mathrm{b}}$ & 479 & 52 \\
\hline 6 & $\mathrm{H}$ & 2-furyl & 405 & $26300^{\mathrm{b}}$ & 489 & 84 \\
\hline 7 & $\mathrm{H}$ & 2-thienyl & 407 & $25000^{\mathrm{b}}$ & 500 & 93 \\
\hline 8 & $\mathrm{H}$ & $\mathrm{COOH}$ & 386 & $15300^{\mathrm{b}}$ & 448 & 62 \\
\hline 9 & $\mathrm{H}$ & Phenyl & 383 & 26100 & 462 & 79 \\
\hline 10 & $\mathrm{CN}$ & 2-benzoxazolyl & 494 & 33200 & 577 & 83 \\
\hline 11 & $\mathrm{CN}$ & 2-benzothiazolyl & 505 & 33100 & 595 & 90 \\
\hline 12 & $\mathrm{CN}$ & $\begin{array}{c}\text { 5-methyl-7-sulfonato-2- } \\
\text { benzoxazolyl }\end{array}$ & 494 & $23000^{\mathrm{b}}$ & 577 & 83 \\
\hline 13 & $\mathrm{CN}$ & 5-chloro-2-benzoxazolyl & 497 & 32400 & 577 & 80 \\
\hline
\end{tabular}




\begin{tabular}{ccccccc}
\hline \multirow{2}{*}{ Compound } & \multirow{2}{*}{$\mathrm{R}^{1}$} & \multirow{2}{*}{$\mathrm{R}^{2}$} & $\begin{array}{c}\lambda_{\mathrm{abs}, \max }, \\
\mathrm{nm}\end{array}$ & $\begin{array}{c}\varepsilon, \\
\mathrm{M}^{-1} \mathrm{~cm}^{-1}\end{array}$ & $\begin{array}{c}\lambda_{\mathrm{em}, \max }, \\
\mathrm{nm}\end{array}$ & $\begin{array}{c}\Delta \lambda^{\mathrm{a}}, \\
\mathrm{nm}\end{array}$ \\
& & & & & \\
\hline $\mathbf{1 4}$ & $\mathrm{CN}$ & 2-benzimidazolyl & 487 & $25400^{\mathrm{b}}$ & $593^{\mathrm{c}}$ & 106 \\
\hline
\end{tabular}

${ }^{\mathrm{a}}$ Stokes Shift, ${ }^{\mathrm{b}}$ in $\mathrm{MeOH},{ }^{\mathrm{c}}$ weak fluorescence

Deligeorgiev et al. ${ }^{[27]}$ prepared the 3-(2-benzothiazolyl)-7-hydroxycoumarin 15 with a sulfonic acid residue $\left(\sigma_{\mathrm{p}}=0.09^{[28]}\right.$ in the Hammett equation) at C-6 (Figure 4). In aqueous solution, this coumarin $\left(\lambda_{\mathrm{abs}, \max }=398 \mathrm{~nm}\right)$ shows an absorption spectrum with a maximum shifted hypsochromically by $41 \mathrm{~nm}$ as compared with the analogous non-sulfonated compound 2 in aqueous borate buffer at $\mathrm{pH}$ 9. In contrast, the position of the fluorescence maximum $\left(\lambda_{\mathrm{em}, \max }=487 \mathrm{~nm}\right)$ stays virtually unchanged, and, as a result, this compound possesses a much larger Stokes shift of $89 \mathrm{~nm}$. In coumarins 16 and 17, extension of the coumarin skeleton by a benzene ring fused to C-5 and C-6 resulted in bathochromic ( 20 $\mathrm{nm}$ ) and bathofluoric (13 and $27 \mathrm{~nm}$, respectively) shifts. The fluorescence QYs for sulphocoumarins 15-17, in water, range from 0.28 for compound $\mathbf{1 5}$ to rather low values of $\sim 0.12$ for benzo[f]coumarins $\mathbf{1 6}$ and $\mathbf{1 7}$.

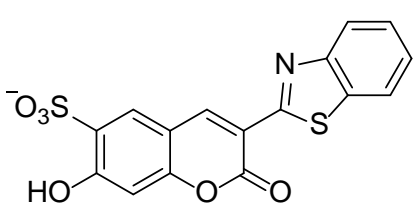

15

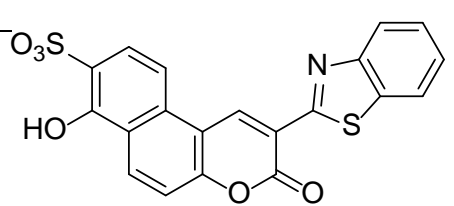

16

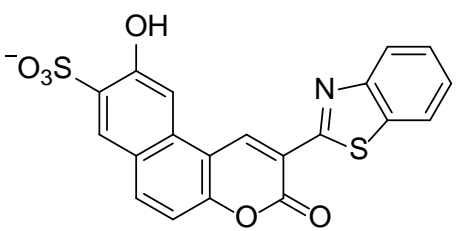

17

Figure 4 3-Benzothiazolylhydroxycoumarins 15-17 with sulphonic acid residues

The absorption specta of 7-hydroxy-3-pyridylcoumarins 18-20 (Figure 5a) have an intense band with maxima located between 388 and $398 \mathrm{~nm}$ in aqueous $\mathrm{NaHCO}_{3}$ (see Table 3). Upon excitation, these compounds emit intense blue light with a maximum at 469-471 nm. Remarkably, the spectra are weakly influenced by the attaching point in the pyridyl substituent and resemble those exhibited by 7-hydroxy-3-phenylcoumarin $\mathbf{9}$. This result indicates the lack of strong direct conjugation between the coumarin and pyridine rings. ${ }^{[29]}$ Quaternization of the pyridine nitrogen causes pronounced bathochromic and bathofluoric shifts in the spectra of the 4-pyridyl isomer 23 (51 and $50 \mathrm{~nm}$, respectively). In the case of 2- and 3-pyridyl isomers quaternized derivatives $\mathbf{2 1}$ and $\mathbf{2 2}$ displayed only small bathochromic shifts $(\sim 20 \mathrm{~nm})$. According to the report, ${ }^{[29]}$ in the case of the 2-iso- 
mer 21 steric constraints may prevent the formation of a planar $\pi$-electron system, whereas for the 3-isomer 22 no mesomeric stabilization is possible. Fluorescence efficiency of quaternized derivatives in aqueous solutions is very low. In contrast to them, quaternized 7-methoxy-3-pyridylcoumarins exhibit much stronger fluorescence. The authors assumed, ${ }^{[29]}$ that the ICT excited states of quaternized 7-hydroxy-3-pyridylcoumarins are better described by a corresponding quinoid structure (Figure $5 b$ ) which is expected to show intense phosphorescence at the expense of fluorescence.

a<smiles>[R]c1cc2ccc(O)cc2oc1=O</smiles><smiles>COc1ccc2cc(-c3cc[n+](C)c(C)c3)c(=O)oc2c1</smiles>

Figure 5 a) 3-Pyridyl-7-hydroxycoumarins 18-23; b) Zwitterion- and quinoid-type resonance structures of compound 23.

Table 3 Absorption and fluorescence maxima of 7-hydroxy-3-pyridylcoumarins and their quaternized derivatives in aq. solution of $\mathrm{NaHCO}_{3}{ }^{[29]}$

\begin{tabular}{cccccc}
\hline Compound & $\mathrm{R}$ & $\lambda_{\mathrm{abs} \text { max }}, \mathrm{nm}$ & $\varepsilon, \mathrm{M}^{-1} \mathrm{~cm}^{-1}$ & $\lambda_{\mathrm{em}, \mathrm{max}}, \mathrm{nm}$ & $\Delta \lambda^{\mathrm{a}}, \mathrm{nm}$ \\
\hline $\mathbf{1 8}$ & 2-pyridyl & 394 & 29000 & 469 & 75 \\
$\mathbf{1 9}$ & 3-pyridyl & 388 & 26800 & 470 & 82 \\
$\mathbf{2 0}$ & 4-pyridyl & 398 & 31700 & 471 & 73 \\
$\mathbf{2 1}$ & 1-methyl-2-pyridinio & 414 & 32700 & 477 & 63 \\
$\mathbf{2 2}$ & 1-methyl-3-pyridinio & 409 & 29400 & 472 & 63 \\
$\mathbf{2 3}$ & 1-methyl-4-pyridinio & 449 & 38000 & 521 & 72 \\
\hline
\end{tabular}

Similar tendencies were observed in the case of 7-aminocoumarins. ${ }^{[30]}$ Quaternized 4pyridylcoumarins 25 and 26 (Figure 6) exhibited the most red-shifted absorption and emission spectra at 482-493 nm and 560-585 nm, respectively, and large Stokes shifts of 78 and $92 \mathrm{~nm}$, whereas coumarin 24 with the 2-pyridinium fragment $\left(\lambda_{\mathrm{abs}, \max } / \lambda_{\mathrm{em}, \max }=\right.$ 
440/494 nm) showed unchanged spectral properties in comparison to those of the non-quaternized analog.

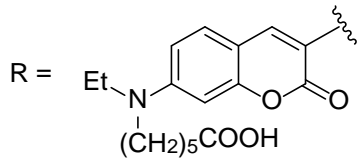

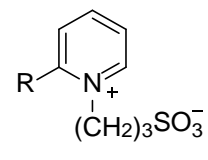

24

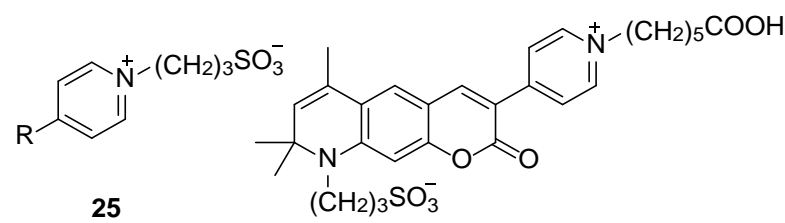

26

Figure 6 7-N,N-Dialkylamino-3-pyridiniumcoumarins 24-26.

In an attempt to develop NIR fluorophores insensitive to thiols and suitable for the imaging of living systems, Richard et al. ${ }^{[31]}$ designed a series of water-soluble 7hydroxycoumarin-hemicyanine hybrids 27-31 (see Figure 7). The extension of the conjugation in the aromatic system of the parent 7-hydroxycoumarin resulted in dramatic bathochromic and bathofluoric shifts. Thus, in aqueous phosphate buffer ( $\mathrm{pH} \mathrm{7.4),} \mathrm{com-}$ pounds 27, 28 and 31 with one double bond $(n=1)$ between 7-hydroxycoumarin and indolium moieties have the absorption and emission maxima at 555-578 nm and 620$643 \mathrm{~nm}$, respectively (Stokes shifts of 63-65 nm). Extension of the conjugation chain by an additional double bond $(n=2)$ had a little effect on the absorption spectra. Absorption maxima of compounds 29 and 30 are found at 564 and $592 \mathrm{~nm}$, respectively. On the other hand, fluorescence spectra turned out to be more sensitive to the number of the double bonds between the coumarin and indolium fragments: emission maxima of $\mathbf{2 9}$ and $\mathbf{3 0}$ are shifted to 720 and $722 \mathrm{~nm}$, respectively, and located already in the IR region of the visible spectrum (this corresponds to large Stokes shifts of 156 and $130 \mathrm{~nm}$ ). However, despite their attractive spectral properties, fluorescence QYs in aqueous media are very low $(<7.8 \%) .{ }^{[31]}$ The introduction of additional negatively charged sulfonate groups preventing dye-dye interactions did not solve the problem of low fluorescence QYs. 

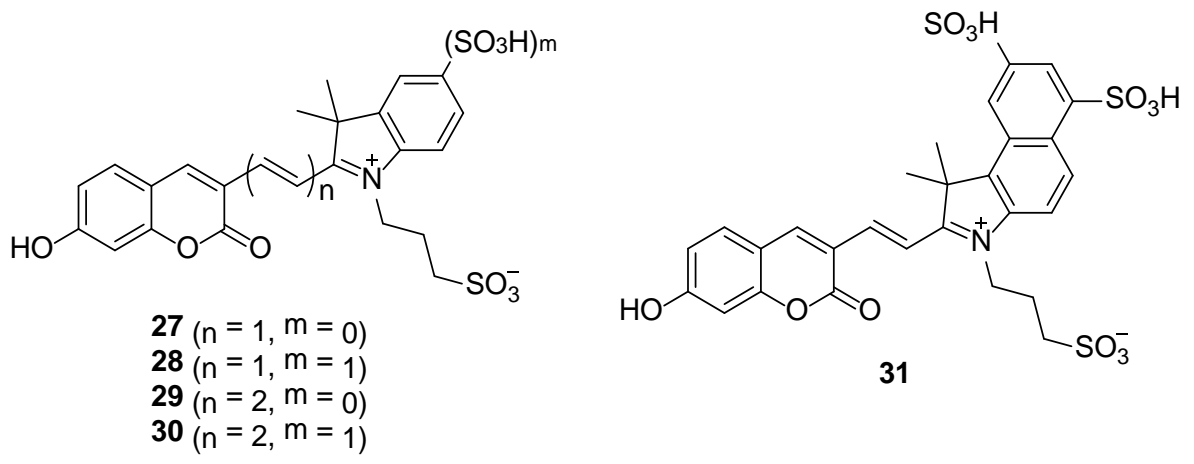

Figure 7 7-Hydroxycoumarin-hemicyanine hybrids 27-31.

Dyomics GmbH used the same approach and designed similar 7-aminocoumarins as “Megastokes"-series of fluorescent dyes. ${ }^{[30]}$ 7-Diethylaminocoumarin-hemicyanine hybrid DY-601XL (Table 1) have the most red-shifted absorption maximum (606 nm in $\mathrm{EtOH})$ in this series and emitted in the near-IR region with a Stokes shift of $57 \mathrm{~nm}$ $\left(\lambda_{\max , \mathrm{em}}=663 \mathrm{~nm}\right)$. The introduction of a trans-double bond between C-3 of the coumarin skeleton and the pyridinium moiety shifted the absorption to $480 \mathrm{~nm}$ (in EtOH) in the case of the 2-pyridinium substituent (compound 32, Figure 8) and to $500 \mathrm{~nm}$ (in EtOH) in the case of the 4-pyridinium substituent (compound 33) in comparison to corresponding analogs without the inserted double bond (coumarins 24 and 25). In commercial dyes DY-480XL and DY-520XL, the introduction of a sulfonic acid residue on the pyridine rings resulted in an additional $\sim 20 \mathrm{~nm}$ bathochromic shift. The presence of the sulfonate groups have an even more pronounced bathofluoric effect on the fluorescence spectra: thus, compounds 32 and 33, which have no sulfonate groups, emit at 600 and $630 \mathrm{~nm}$ (Stokes shifts of 120 and $130 \mathrm{~nm}$ ), respectively, whereas DY-480XL and DY-520XL - at 630 and $664 \mathrm{~nm}$ (Stokes shifts of 130 and $144 \mathrm{~nm}$ ) in EtOH. The replacement of the pyridinium fragment with a quinolinium moiety in compounds 34-36 further shifted the absorption and fluorescence spectra towards the IR region and increased Stokes shifts to huge values of up to $160 \mathrm{~nm}$ (see Table 4 for further details). Closely related "clickable" fluorophores $\mathbf{3 7}$ and $\mathbf{3 8}$ were proposed for applications in (bio)orthogonal labeling schemes. $^{[32]}$ 


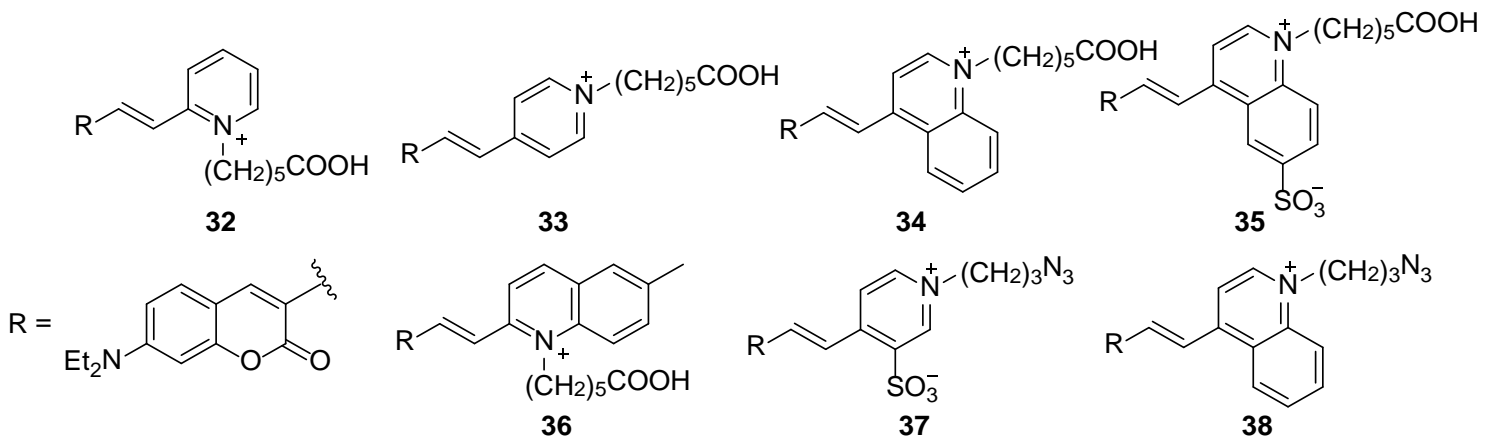

Figure 8 7-N,N-Diethylamino-3-vinylcoumarins 32-38.

Table 4 Spectral properties of 7-dimethylamino-3-vinylcoumarins 32-38.

\begin{tabular}{cccccc}
\hline Compound & $\lambda_{\text {abs,max }}, \mathrm{nm}$ & $\varepsilon, \mathrm{M}^{-1} \mathrm{~cm}^{-1}$ & $\lambda_{\text {em,max }}, \mathrm{nm}$ & $\Delta \lambda^{\mathrm{a}}, \mathrm{nm}$ & Solvent \\
\hline $\mathbf{3 2}$ & 480 & - & 600 & 120 & $\mathrm{EtOH}$ \\
$\mathbf{3 4}$ & 500 & - & 630 & 130 & $\mathrm{EtOH}$ \\
$\mathbf{3 5}$ & 540 & - & 695 & 155 & $\mathrm{MeOH}$ \\
$\mathbf{3 6}$ & 555 & - & 715 & 160 & $\mathrm{MeOH}$ \\
$\mathbf{3 7}$ & 520 & - & 655 & 135 & $\mathrm{MeOH}$ \\
$\mathbf{3 8}^{[32 \mathrm{c}]}$ & 544 & 53000 & 675 & 131 & $\mathrm{PBS}$ \\
& 549 & 27000 & 712 & 158 & $\mathrm{MeOH}$ \\
\hline
\end{tabular}

In contrast to 7-hydroxycoumarins, 7-aminocoumarins do not exhibit significant $\mathrm{pH}$ sensitivity and are highly fluorescent over a wide range of $\mathrm{pH}$ values in their neutral forms. Methods of their synthesis and their photophysical properties were reviewed. ${ }^{[33]}$ The parent compound 7-aminocoumarin has the main band at $380 \mathrm{~nm}(\varepsilon=$ $\left.18000 \mathrm{M}^{-1} \mathrm{~cm}^{-1}\right)$ in water and the emission maximum at $444 \mathrm{~nm}\left(\Phi_{\mathrm{fl}}=0.55^{[34]}\right.$, Stokes shift of $64 \mathrm{~nm}$ ). Amino groups, in particular if they are not or only partially alkylated are capable of forming hydrogen bonds with molecules of water. Therefore, simple 7-aminocoumarins are slightly soluble in water. ${ }^{[21 \mathrm{~b}]}$ Absorption and emission maxima of 7-aminocoumarins slightly shift to longer wavelengths upon increasing the degree of alkylation of 
the amino group. ${ }^{[21 \mathrm{a}]}$ A larger shift toward the red can be obtained through substitution with heteroaryl and trifluoromethyl groups in positions 3 and 4, respectively. Thus, the 4trifluoromethyl group in laser dyes 39 (Coumarin 151) and 40 (Coumarin 307) provides bathochromic (28 and $29 \mathrm{~nm}$, respectively, in EtOH) and bathofluoric (50 and $55 \mathrm{~nm}$ ) shifts relative to analogous compounds 41 (Coumarin 120) and 42 (Coumarin 2) in the same solvent (see Figure 9). In addition, the introduction of a trifluoromethyl group at C4 was found to reduce photobleaching by $20 \%$ in coumarin dyes. ${ }^{[21 c]}$ The benzothiazole group in dye 47 (Coumarin 6) causes even larger shifts of $85 \mathrm{~nm}$ (in the absorption maximum) and $60 \mathrm{~nm}$ (in the emission maximum) and increases the extinction coefficient as compared with compound $\mathbf{4 4}$ (Coumarin 1).<smiles>Nc1ccc2c(C(F)(F)F)cc(=O)oc2c1</smiles>

39 (Coumarin 151)<smiles>CCNc1cc2oc(=O)cc(C(F)(F)F)c2cc1C</smiles>

40 (Coumarin 307)<smiles>Cc1cc(=O)oc2cc(N)ccc12</smiles>

41 (Coumarin 120)<smiles>CCNc1cc2oc(=O)cc(C)c2cc1C</smiles>

42 (Coumarin 2)<smiles>CCOC(=O)c1cc2ccc(N(C)C)cc2oc1=O</smiles>

43 (Coumarin 14)<smiles>CCNc1ccc2c(C)cc(=O)oc2c1</smiles><smiles>CN(C)c1ccc2c(C(F)(F)F)cc(=O)oc2c1</smiles><smiles>CCNc1ccc2c(C(F)(F)F)cc(=O)oc2c1</smiles>

45 (Coumarin 152)<smiles>CCN(CC)c1ccc2cc(-c3nc4ccccc4s3)c(=O)oc2c1</smiles>

47 (Coumarin 6)<smiles>O=C(O)c1cc2cc3c4c(c2oc1=O)CCCN4CCC3</smiles>

48 (Coumarin 343)<smiles>O=c1ccc2cc3c4c(c2o1)CCCN4CCC3</smiles>

49 (Coumarin 6H)

Figure 9 Coumarin laser dyes 39-49.

As a rule, aminocoumarins have lower fluorescence efficiencies in highly polar solvents. This reduction is particularly sharp if the amino group at the position 7 is dialkylated (such amino groups cannot form hydrogen bonds with solvent molecules). It turns out that upon exciting the dyes with less rigid geometries, the rotation of the amino function facilitates an internal conversion of the initial ICT state to a non-emissive twisted chargetransfer (TICT) state with full charge separation (see Figure 10). ${ }^{[21 \mathrm{~d}, 21 \mathrm{e}, 21 \mathrm{~g}, 35]}$ This state is stabilized by electron-withdrawing groups at C-3 or C-4 and by electrostatic interaction with molecules of a polar solvent. As a result, coumarins 43, 45 and 46 have poor fluorescence QYs in aqueous and alcoholic media. As expected, rigidization of the amino group 
by incorporation into one or two six-membered rings as in compounds 48 and 49 led to a considerable improvement of the fluorescence efficiency, since in this case excitation yields a normal planar ICT state. It was also found that there is no need for rigidization if the amino group carries only one alkyl group. The rigidized amino groups are more strongly electron-donating than dimethyl- or diethylamino groups, therefore they shift absorption and emission bands further to longer wavelengths. The absorption and emission maxima of laser dye 48 (Coumarin 343) with a carboxylic group at C-3 shift $40 \mathrm{~nm}$ towards the blue upon addition of a base, which is consistent with a less withdrawing ability of carboxylate compared with non-ionized carboxylic group. ${ }^{\text {[21a] }}$
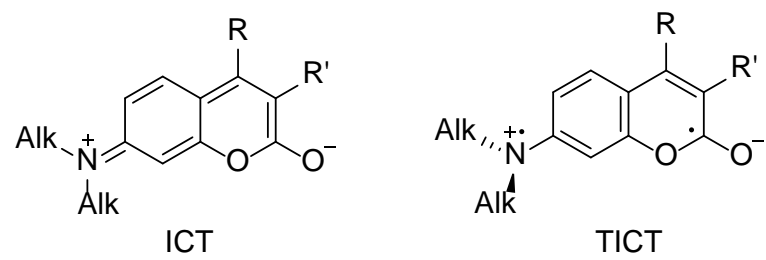

Figure 10 "Normal" intramolecular charge transfer (ICT) and twisted intramolecular charge transfer (TICT) excited states of $7-N, N$-dialkylaminocoumarins.

To investigate the influence of the substitution at C-3 in laser dye 49 (Coumarin 6H) on lasing characterics, coumarins 50-58 were prepared. ${ }^{[21 f]}$ Variation of the functional group at $\mathrm{C}-3$ from $\mathrm{H}$ to phenyl and other residues caused a red shift in wavelengths of the absorption and fluorescence maxima. Benzazole substituents (compounds 50-52 in Table 5) provided the largest bathochromic shift, but at the same time reduced the Stokes shift almost by a factor of two relative to the unsubstituted compound 49. Phenyl and pyridyl substituents (compounds 54, 56-58) moderately shifted absorption and emission bands toward the red spectral region retaining relatively large values of Stokes shifts (60-78 nm). Sulfonyl groups in coumarins $\mathbf{5 3}$ and $\mathbf{5 5}$ had an effect on spectral properties which is between the effects of the benzoxazolyl and pyridyl substituents.<smiles>[R]c1cc2cc3c4c(c2oc1=O)CCCN4CCC3</smiles>

50-58

Figure 11 3-Substituted analogs of laser dye Coumarin 6H 50-58. 
Table 5 Spectral properties of coumarins $\mathbf{4 9 - 5 8}$ in aq. EtOH (1:1).

\begin{tabular}{cccccc}
\hline Compound & R & $\lambda_{\text {abs,max }}, \mathrm{nm}$ & $\lambda_{\mathrm{em}, \mathrm{max}}, \mathrm{nm}$ & $\Delta \lambda, \mathrm{nm}$ & $\Phi_{\mathrm{fl}}$ \\
\hline $\mathbf{4 9}$ & $\mathrm{H}$ & 404 & 488 & 84 & 0.96 \\
$\mathbf{5 0}$ & 2-benzimidazolyl & 473 & 512 & 39 & 1.00 \\
$\mathbf{5 1}$ & 2-benzothiazolyl & 490 & 526 & 36 & 0.96 \\
$\mathbf{5 2}$ & 2-benzoxazolyl & 474 & 512 & 38 & 0.89 \\
$\mathbf{5 3}$ & methylsulfonyl & 442 & 488 & 46 & 0.87 \\
$\mathbf{5 4}$ & phenyl & 424 & 502 & 78 & 0.95 \\
$\mathbf{5 5}$ & phenylsulfonyl & 450 & 492 & 42 & 1.00 \\
$\mathbf{5 6}$ & 2-pyridyl & 442 & 502 & 60 & 0.88 \\
$\mathbf{5 7}$ & 3-pyridyl & 433 & 506 & 73 & 0.98 \\
$\mathbf{5 8}$ & 4-pyridyl & 445 & 508 & 63 & 0.88 \\
& & & & & \\
\hline
\end{tabular}

7-Aminothieno[3,2-c]coumarins (see Figure 12) contain a thiophene ring fused along positions 2 and 3 with the coumarin scaffold. The fused compounds 59 and $\mathbf{6 0}$ absorb violet light with maxima at 404 and $395 \mathrm{~nm}$ and emit blue light at 480 and $486 \mathrm{~nm}$ with large quantum efficiencies (0.82 and 1.00 , respectively) in MeCN. ${ }^{[36]}$ The Stokes shifts (76 nm and $91 \mathrm{~nm}$ ) were found to be somewhat larger than the Stokes shift for 7-diethylamino-4-methylcoumarin $\left(63 \mathrm{~nm}^{[21 \mathrm{~g}]}\right)$ in the same solvent.

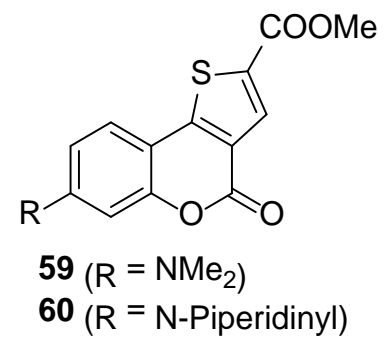

Figure 12 7-Aminothieno[3,2-c]coumarins. 
Replacement of the methyl group at C-4 in compounds 44 and 151 with an amino group led to coumarins 61-69, 134 and 135 (see Figure 13 and Table 6), and this was accompanied by a hypsochromic shift in the absorption bands by $10-15 \mathrm{~nm}$ and by a hypsofluoric shift of the emission bands by approximately $20-30 \mathrm{~nm}^{[37]}$ The authors explained this phenomenon with an increased charge transfer along the conjugation chain $4-\mathrm{R} \rightarrow$ $\mathrm{C}(4)=\mathrm{C}(3)$ which opposes the main conjugative interaction in the system $7-\mathrm{NR}_{2} \rightarrow$ $\mathrm{C}(2)=\mathrm{O}$. Interestingly, substitution of the 4-monoalkylamino group by a more electrondonating 4-dialkylamino group in a transition series from compounds 62-65, 70 and 71 to coumarins 66-69 and 72-74 induces a bathochromic shift of the absorption band by 7-19 $\mathrm{nm}$, although the transition from 4-aminocoumarin 61 to $4-N$-monoalkylaminocoumarins 62-65 does not produce any significant influence. According to the report, the reason for this discrepancy is the steric interaction between the 4-dialkylamino group and the $5-\mathrm{H}$ atom which weakens $\mathrm{p}-\pi$ conjugation in the $\mathrm{N}-\mathrm{C}(4)=\mathrm{C}(3)-\mathrm{C}(2)=\mathrm{O}$ system. This effect is even more pronounced when the alkyl groups form a ring as in compounds 68 and 69. Their absorption maxima are further bathochromically shifted by approximately $10 \mathrm{~nm}$ in comparison to coumarins $\mathbf{6 6}$ and $\mathbf{6 7}$. Introduction of a 3-alkyl substituent further increases the steric hindrance and has a similar effect on the absorption maximum. Thus, compounds 70-74 have the main absorption bands shifted bathochromically by 6-13 nm compared to 63, 64 and 69. The positions of emission bands are influenced by similar effects as are the absorption spectra. One important exception is compound 62 which has a fluorescence maximum shifted by $30 \mathrm{~nm}$ towards longer wavelengths compared to compounds 63-65. This behavior was attributed to steric hindrance between the t-butyl group and the $3-\mathrm{H}$ atom in the excited state $\mathrm{S}_{1}$ of coumarin 62 . Therefore, the degree of conjugation between the 4-substituent and the rest of the molecule is reduced. Dialkylamino groups possess greater vibrational degrees of freedom in comparison with morpholine, piperidine and monoalkylamino groups. This increases the probability of energy dissipation from the $\mathrm{S}_{1}$ along nonradiative pathways. As a consequence, 4- $N, N$-dialkylaminocoumarins 66, 67 and 76 exhibit lower emission efficiencies in EtOH than their analogs 61-65, 68-75 and 77. 
<smiles>[R]c1ccc2c([R1])c([R])c(=O)oc2c1</smiles>

44, 61-96, 97-98, 101-133<smiles>CCNc1cc2oc(=O)cc(C)c2cc1F</smiles>

99

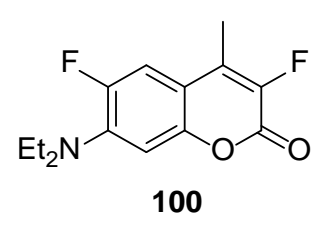

100

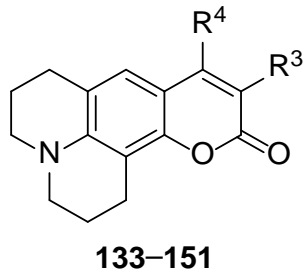

Figure 13 Substituted 7-aminocoumarins 44, 61-151.

Table 6 Spectral properties of coumarins 44, 61-151 in EtOH.

\begin{tabular}{|c|c|c|c|c|c|c|c|}
\hline & $\mathrm{R}^{3}$ & $\mathrm{R}^{4}$ & $\mathrm{R}^{7}$ & $\begin{array}{c}\lambda_{\text {abs,max }}, \\
n m\end{array}$ & $\begin{array}{c}\lambda_{\text {em,max }}, \\
n m\end{array}$ & $\begin{array}{l}\Delta \lambda, \\
\mathrm{nm}\end{array}$ & $\Phi_{\mathrm{fl}}$ \\
\hline 44 & $\mathrm{H}$ & $\mathrm{Me}$ & $\mathrm{NEt}_{2}$ & 377 & 455 & 78 & 0.68 \\
\hline 61 & $\mathrm{H}$ & $\mathrm{NH}_{2}$ & $\mathrm{NEt}_{2}$ & 350 & 410 & 60 & 0.58 \\
\hline 62 & $\mathrm{H}$ & $\mathrm{NH} t \mathrm{Bu}$ & $\mathrm{NEt}_{2}$ & 349 & 445 & 96 & 0.62 \\
\hline 63 & $\mathrm{H}$ & NHCy & $\mathrm{NEt}_{2}$ & 349 & 414 & 65 & 0.67 \\
\hline 64 & $\mathrm{H}$ & NHBn & $\mathrm{NEt}_{2}$ & 350 & 410 & 60 & 0.68 \\
\hline 65 & $\mathrm{H}$ & $\mathrm{NHCH}_{2} \mathrm{CH}_{2} \mathrm{OH}$ & $\mathrm{NEt}_{2}$ & 349 & 412 & 63 & 0.63 \\
\hline 66 & $\mathrm{H}$ & $\mathrm{NEt}_{2}$ & $\mathrm{NEt}_{2}$ & 356 & 440 & 84 & $<0.10$ \\
\hline 67 & $\mathrm{H}$ & $\mathrm{NBu}_{2}$ & $\mathrm{NEt}_{2}$ & 358 & 440 & 82 & 0.15 \\
\hline 68 & $\mathrm{H}$ & $N$-piperidyl & $\mathrm{NEt}_{2}$ & 365 & 447 & 82 & 0.36 \\
\hline 69 & $\mathrm{H}$ & $N$-morpholyl & $\mathrm{NEt}_{2}$ & 365 & 445 & 80 & 0.70 \\
\hline 70 & Сy & NHCy & $\mathrm{NEt}_{2}$ & 363 & 440 & 77 & 0.33 \\
\hline 71 & $\mathrm{Bn}$ & NHBn & $\mathrm{NEt}_{2}$ & 356 & 435 & 79 & 0.31 \\
\hline 72 & Et & $N$-morpholyl & $\mathrm{NEt}_{2}$ & 375 & 465 & 90 & 0.39 \\
\hline 73 & Сy & $N$-morpholyl & $\mathrm{NEt}_{2}$ & 376 & 464 & 88 & 0.76 \\
\hline 74 & $\mathrm{Bn}$ & $N$-morpholyl & $\mathrm{NEt}_{2}$ & 378 & 466 & 88 & 0.65 \\
\hline
\end{tabular}




\begin{tabular}{|c|c|c|c|c|c|c|c|}
\hline & $\mathrm{R}^{3}$ & $\mathrm{R}^{4}$ & $\mathrm{R}^{7}$ & $\begin{array}{c}\lambda_{\text {abs,max }}, \\
\mathrm{nm}\end{array}$ & $\begin{array}{c}\lambda_{\mathrm{em}, \max } \\
\mathrm{nm}\end{array}$ & $\begin{array}{l}\Delta \lambda, \\
\mathrm{nm}\end{array}$ & $\Phi_{\mathrm{fl}}$ \\
\hline 75 & $\mathrm{H}$ & $N$-morpholyl & $\begin{array}{l}N \text {-pi- } \\
\text { peridyl }\end{array}$ & 360 & 455 & 95 & 0.49 \\
\hline 76 & $\mathrm{H}$ & $\mathrm{NEt}_{2}$ & $\begin{array}{l}N \text {-mor- } \\
\text { pholyl }\end{array}$ & 344 & 440 & 96 & $<0.10$ \\
\hline 77 & $\mathrm{H}$ & $N$-morpholyl & $\begin{array}{l}N \text {-mor- } \\
\text { pholyl }\end{array}$ & 350 & 450 & 100 & 0.31 \\
\hline 78 & $\mathrm{Cl}$ & $\mathrm{Me}$ & $\mathrm{NEt}_{2}$ & 388 & 476 & 88 & 0.81 \\
\hline 79 & $\mathrm{Br}$ & $\mathrm{Me}$ & $\mathrm{NEt}_{2}$ & 390 & 478 & 88 & 0.51 \\
\hline 80 & $\mathrm{Cl}$ & $\mathrm{H}$ & $\mathrm{NEt}_{2}$ & 398 & 485 & 87 & 0.69 \\
\hline 81 & $\mathrm{Br}$ & $\mathrm{H}$ & $\mathrm{NEt}_{2}$ & 400 & 482 & 82 & 0.50 \\
\hline 82 & $\mathrm{I}$ & $\mathrm{H}$ & $\mathrm{NEt}_{2}$ & 400 & - & - & - \\
\hline 83 & $\mathrm{Cl}$ & NHBn & $\mathrm{NEt}_{2}$ & 365 & 450 & 85 & 0.32 \\
\hline 84 & $\mathrm{Br}$ & NHBn & $\mathrm{NEt}_{2}$ & 365 & 450 & 85 & 0.23 \\
\hline 85 & $\mathrm{Cl}$ & $N$-morpholyl & $\mathrm{NEt}_{2}$ & 384 & 476 & 92 & 0.22 \\
\hline 86 & $\mathrm{Br}$ & $N$-morpholyl & $\mathrm{NEt}_{2}$ & 386 & 480 & 94 & 0.10 \\
\hline 87 & I & $N$-morpholyl & $\mathrm{NEt}_{2}$ & 391 & - & - & - \\
\hline 88 & $\mathrm{Cl}$ & $\mathrm{Cl}$ & $\mathrm{NEt}_{2}$ & 402 & 490 & 88 & 0.10 \\
\hline 89 & $\mathrm{Br}$ & $\mathrm{Cl}$ & $\mathrm{NEt}_{2}$ & 405 & 490 & 85 & $<0.10$ \\
\hline 90 & $\mathrm{I}$ & $\mathrm{Cl}$ & $\mathrm{NEt}_{2}$ & 406 & - & - & - \\
\hline 91 & $\mathrm{Cl}$ & $\mathrm{CH}\left(\mathrm{COCH}_{3}\right)_{2}$ & $\mathrm{NEt}_{2}$ & 405 & 490 & 85 & 0.41 \\
\hline 92 & $\mathrm{Br}$ & $\mathrm{CH}\left(\mathrm{COCH}_{3}\right)_{2}$ & $\mathrm{NEt}_{2}$ & 408 & 490 & 82 & 0.24 \\
\hline 93 & $\mathrm{I}$ & $\mathrm{CH}\left(\mathrm{COCH}_{3}\right)_{2}$ & $\mathrm{NEt}_{2}$ & 408 & - & - & - \\
\hline
\end{tabular}




\begin{tabular}{|c|c|c|c|c|c|c|c|}
\hline & $\mathrm{R}^{3}$ & $\mathrm{R}^{4}$ & $\mathrm{R}^{7}$ & $\begin{array}{c}\lambda_{\text {abs,max }}, \\
n m\end{array}$ & $\begin{array}{c}\lambda_{\mathrm{em}, \max }, \\
\mathrm{nm}\end{array}$ & $\begin{array}{l}\Delta \lambda, \\
\mathrm{nm}\end{array}$ & $\Phi_{\mathrm{fl}}$ \\
\hline 94 & $\mathrm{Cl}$ & $\mathrm{Me}$ & $\mathrm{NH}_{2}$ & 368 & 457 & 89 & 0.61 \\
\hline 95 & $\mathrm{Br}$ & $\mathrm{Me}$ & $\mathrm{NH}_{2}$ & 370 & 460 & 90 & 0.56 \\
\hline 96 & I & $\mathrm{Me}$ & $\mathrm{NH}_{2}$ & 380 & - & - & - \\
\hline 97 & $\mathrm{~F}$ & $\mathrm{H}$ & $\mathrm{NEt}_{2}$ & 382 & 485 & 103 & 0.59 \\
\hline 98 & $\mathrm{~F}$ & $\mathrm{Me}$ & $\mathrm{NEt}_{2}$ & 376 & 476 & 100 & 0.75 \\
\hline 99 & $\mathrm{H}$ & $\mathrm{Me}$ & $\mathrm{NEt}_{2}$ & 368 & 450 & 82 & $<0.10$ \\
\hline 100 & $\mathrm{~F}$ & $\mathrm{Me}$ & $\mathrm{NEt}_{2}$ & 362 & 480 & 118 & $<0.10$ \\
\hline 101 & $\mathrm{CHO}$ & $\mathrm{H}$ & $\mathrm{NEt}_{2}$ & 446 & 494 & 48 & $<0.10$ \\
\hline 102 & $\mathrm{CHO}$ & $\mathrm{Me}$ & $\mathrm{NEt}_{2}$ & 436 & 490 & 54 & $<0.10$ \\
\hline 103 & $\mathrm{CHO}$ & $\mathrm{Cl}$ & $\mathrm{NEt}_{2}$ & 449 & 502 & 53 & $<0.10$ \\
\hline 104 & $\mathrm{CHO}$ & $N$-morpholyl & $\mathrm{NEt}_{2}$ & 409 & 475 & 66 & $<0.10$ \\
\hline 105 & $\mathrm{CHO}$ & NHBn & $\mathrm{NEt}_{2}$ & 379 & 460 & 81 & $<0.10$ \\
\hline 106 & $\mathrm{CHO}$ & $\mathrm{Cl}$ & $\begin{array}{l}N \text {-mor- } \\
\text { pholyl }\end{array}$ & 435 & 500 & 65 & $<0.10$ \\
\hline 107 & $\mathrm{CHO}$ & $N$-morpholyl & $\begin{array}{l}N \text {-mor- } \\
\text { pholyl }\end{array}$ & 392 & 480 & 88 & $<0.10$ \\
\hline 108 & $\mathrm{H}$ & $N$-imidazolyl & $\mathrm{NEt}_{2}$ & 394 & 480 & 86 & $<0.10$ \\
\hline 109 & $\mathrm{H}$ & $N$-benzimidazolyl & $\mathrm{NEt}_{2}$ & 399 & 480 & 81 & $<0.10$ \\
\hline 110 & $\mathrm{H}$ & $\begin{array}{c}\text { diethylamino- } \\
\text { methyl }\end{array}$ & $\mathrm{NEt}_{2}$ & 379 & 468 & 89 & $<0.10$ \\
\hline 111 & $\mathrm{H}$ & $\begin{array}{c}\text { piperidin-1- } \\
\text { ylmethyl }\end{array}$ & $\mathrm{NEt}_{2}$ & 382 & 465 & 83 & $<0.10$ \\
\hline
\end{tabular}




\begin{tabular}{|c|c|c|c|c|c|c|c|}
\hline & $\mathrm{R}^{3}$ & $\mathrm{R}^{4}$ & $\mathrm{R}^{7}$ & $\begin{array}{c}\lambda_{\text {abs,max }}, \\
n m\end{array}$ & $\begin{array}{c}\lambda_{\text {em,max }}, \\
n m\end{array}$ & $\begin{array}{l}\Delta \lambda, \\
\mathrm{nm}\end{array}$ & $\Phi_{\mathrm{fl}}$ \\
\hline 112 & $\mathrm{H}$ & $\begin{array}{c}\text { morpholin-4-yl- } \\
\text { methyl }\end{array}$ & $\mathrm{NEt}_{2}$ & 384 & 470 & 86 & 0.35 \\
\hline 113 & $\mathrm{H}$ & $\begin{array}{l}\text { imidazol-1-yl- } \\
\text { methyl }\end{array}$ & $\mathrm{NEt}_{2}$ & 378 & 480 & 102 & 0.28 \\
\hline 114 & $\mathrm{H}$ & $\begin{array}{c}\text { benzimidazol-1- } \\
\text { yl-methyl }\end{array}$ & $\mathrm{NEt}_{2}$ & 385 & 480 & 95 & 0.22 \\
\hline 115 & $N$-piperidyl & $\mathrm{Me}$ & $\mathrm{NEt}_{2}$ & 378 & 490 & 112 & $<0.10$ \\
\hline 116 & $N$-morpholyl & $\mathrm{Me}$ & $\mathrm{NEt}_{2}$ & 377 & 480 & 103 & $<0.10$ \\
\hline 117 & $\mathrm{NH}_{2}$ & $\mathrm{Me}$ & $\mathrm{NEt}_{2}$ & 398 & 490 & 92 & 0.95 \\
\hline \multirow{2}{*}{118} & $\mathrm{Ph}$ & $\mathrm{H}$ & $\mathrm{NEt}_{2}$ & 401 & 484 & 83 & 0.92 \\
\hline & $\mathrm{Ph}$ & $\mathrm{H}$ & $\mathrm{NEt}_{2}$ & 397 & 480 & 83 & $0.73^{\mathrm{a}}$ \\
\hline 119 & $\mathrm{Ph}$ & $\mathrm{Me}$ & $\mathrm{NEt}_{2}$ & 383 & 475 & 92 & 0.86 \\
\hline 120 & p-tolyl & $\mathrm{Me}$ & $\mathrm{NEt}_{2}$ & 384 & 468 & 84 & 0.75 \\
\hline 121 & $o$-tolyl & $\mathrm{Me}$ & $\mathrm{NEt}_{2}$ & 383 & 454 & 71 & 1.00 \\
\hline 122 & $\begin{array}{c}\text { p-phenoxy- } \\
\text { phenyl }\end{array}$ & $\mathrm{Me}$ & $\mathrm{NEt}_{2}$ & 385 & 464 & 79 & 0.95 \\
\hline 123 & $p$-chlorophenyl & $\mathrm{Me}$ & $\mathrm{NEt}_{2}$ & 388 & 472 & 84 & 0.53 \\
\hline 124 & o-chlorophenyl & $\mathrm{Me}$ & $\mathrm{NEt}_{2}$ & 385 & 462 & 77 & 0.92 \\
\hline 125 & $p$-fluorophenyl & $\mathrm{Me}$ & $\mathrm{NEt}_{2}$ & 386 & 465 & 79 & 0.97 \\
\hline 126 & $o$-fluorophenyl & $\mathrm{Me}$ & $\mathrm{NEt}_{2}$ & 384 & 475 & 91 & 0.78 \\
\hline 127 & p-cyanophenyl & $\mathrm{Me}$ & $\mathrm{NEt}_{2}$ & 394 & 485 & 91 & 0.70 \\
\hline 128 & o-cyanophenyl & $\mathrm{Me}$ & $\mathrm{NEt}_{2}$ & 393 & 475 & 82 & 0.66 \\
\hline
\end{tabular}




\begin{tabular}{|c|c|c|c|c|c|c|c|}
\hline & $\mathrm{R}^{3}$ & $\mathrm{R}^{4}$ & $\mathrm{R}^{7}$ & $\begin{array}{c}\lambda_{\mathrm{abs}, \max }, \\
\mathrm{nm}\end{array}$ & $\begin{array}{c}\lambda_{\mathrm{em}, \max }, \\
\mathrm{nm}\end{array}$ & $\begin{array}{l}\Delta \lambda, \\
\mathrm{nm}\end{array}$ & $\Phi_{\mathrm{fl}}$ \\
\hline 129 & $\mathrm{Ph}$ & $N$-morpholyl & $\mathrm{NEt}_{2}$ & 382 & 470 & 88 & $<0.10$ \\
\hline 130 & $\mathrm{Ph}$ & $\mathrm{Cl}$ & $\mathrm{NEt}_{2}$ & 397 & 490 & 93 & $<0.10$ \\
\hline 131 & $\mathrm{Ph}$ & $\mathrm{CF}_{3}$ & $\mathrm{NEt}_{2}$ & 415 & 545 & 130 & $<0.10$ \\
\hline 132 & $\beta$-styryl & $\mathrm{H}$ & $\mathrm{NEt}_{2}$ & 421 & 477 & 56 & $0.85^{\mathrm{a}}$ \\
\hline 133 & $\mathrm{H}$ & $\mathrm{H}$ & $\mathrm{NEt}_{2}$ & 380 & 462 & 82 & 0.29 \\
\hline 134 & $\mathrm{H}$ & $\mathrm{NEt}_{2}$ & - & 372 & 467 & 95 & 0.89 \\
\hline 135 & $\mathrm{H}$ & $N$-morpholyl & - & 382 & 466 & 84 & 1.00 \\
\hline 136 & $\mathrm{Cl}$ & $\mathrm{Me}$ & - & 404 & 495 & 91 & 0.95 \\
\hline 137 & $\mathrm{Br}$ & $\mathrm{Me}$ & - & 404 & 485 & 81 & 0.65 \\
\hline 138 & I & $\mathrm{Me}$ & - & 406 & - & - & - \\
\hline 139 & $\mathrm{Cl}$ & $\mathrm{Cl}$ & - & 424 & 500 & 76 & $<0.10$ \\
\hline 140 & $\mathrm{Br}$ & $\mathrm{Cl}$ & - & 420 & 500 & 80 & $<0.10$ \\
\hline 141 & I & $\mathrm{Cl}$ & - & 423 & - & - & - \\
\hline 142 & $\mathrm{~F}$ & $\mathrm{H}$ & - & 392 & 505 & 113 & 0.72 \\
\hline 143 & $\mathrm{~F}$ & $\mathrm{Me}$ & - & 403 & 495 & 92 & 0.83 \\
\hline 144 & $\mathrm{CHO}$ & $\mathrm{H}$ & - & 469 & 510 & 41 & 0.48 \\
\hline 145 & $\mathrm{CHO}$ & $\mathrm{Me}$ & - & 454 & 508 & 54 & 0.81 \\
\hline 146 & $\mathrm{CHO}$ & $\mathrm{Cl}$ & - & 468 & 520 & 52 & $<0.10$ \\
\hline 147 & $\mathrm{H}$ & $N$-imidazolyl & - & 415 & 495 & 80 & 0.63 \\
\hline 148 & $\mathrm{H}$ & $\begin{array}{c}\text { morpholin-4-yl- } \\
\text { methyl }\end{array}$ & - & 400 & 490 & 90 & 1.00 \\
\hline
\end{tabular}




\begin{tabular}{|c|c|c|c|c|c|c|c|}
\hline & $\mathrm{R}^{3}$ & $\mathrm{R}^{4}$ & $\mathrm{R}^{7}$ & $\begin{array}{c}\lambda_{\text {abs,max }}, \\
n m\end{array}$ & $\begin{array}{c}\lambda_{\text {em,max }}, \\
n m\end{array}$ & $\begin{array}{l}\Delta \lambda, \\
\mathrm{nm}\end{array}$ & $\Phi_{\mathrm{fl}}$ \\
\hline 149 & $\mathrm{H}$ & $\begin{array}{l}\text { imidazol-1-yl- } \\
\text { methyl }\end{array}$ & - & 400 & 500 & 100 & 0.83 \\
\hline 150 & $\mathrm{H}$ & $N$-morpholyl & - & 398 & 490 & 92 & 0.95 \\
\hline 151 & $\mathrm{H}$ & $\mathrm{Me}$ & - & 394 & 474 & 80 & 0.86 \\
\hline
\end{tabular}

The introduction of a halogen atom into the position 3 of the 7-aminocoumarin scaffold causes small bathochromic shifts of 10-25 nm relative to the corresponding unsubstituted coumarins. ${ }^{[38]}$ The absorption spectra of coumarins 78-93 and 136-141 (Table 6) are not very sensitive to the nature of the halogen atom at C-3. In the row from chlorine to iodine, the absorption maximum varies within a small interval $(\Delta \lambda=0-5 \mathrm{~nm})$. However, emission bands of 3-chloro- and 3-bromocoumarins 78-81, 83-86, 88, 89, 91, 92, 94, 95, 136, 137, 139 and 140 are shifted bathofluorically by 10-45 $\mathrm{nm}$ compared with their analogs without the substituent at C-3. As expected, due to the quenching effect of the heavy atom, fluorescence efficiency drops in the series $\mathrm{Cl} \rightarrow \mathrm{Br} \rightarrow \mathrm{I}$. Interestingly, the chlorine

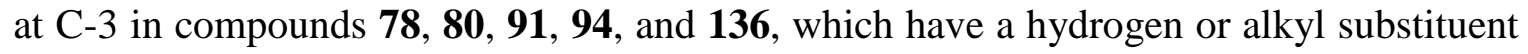
at C-4, enhances the fluorescence intensity compared to the analogous 3-unsubstituted coumarins. In contrast, in the case of coumarins 83, 85, 88 and 139, substituents exhibiting positive mesomeric effects (amino groups or chlorine) at C-4 quench the emission. A decrease in the electron-donating ability of the substituent at C-4 in the series $\mathrm{NHBn} \rightarrow \mathrm{N}\left(\mathrm{CH}_{2} \mathrm{CH}_{2}\right)_{2} \mathrm{O} \rightarrow \mathrm{Me} \rightarrow \mathrm{H} \rightarrow \mathrm{Cl} \rightarrow \mathrm{CH}\left(\mathrm{COCH}_{3}\right)_{2}$ is accompanied by bathochromic and bathofluoric shifts.

In comparison to other 3-halo-7-aminocoumarins, 3-fluoro derivatives $(\mathbf{9 7 ,}, \mathbf{9 8 ,} \mathbf{1 4 2 ,} 143)$ have the long-wavelength absorption maximum hypsochromically shifted by approximately $15 \mathrm{~nm}$, thus resembling the spectral characteristics of 3-unsubstituted analogs. ${ }^{[39]}$ In the series of halogens, the fluorine atom participates most effectively in $\mathrm{p}-\pi$ conjugation, hindering the charge transfer from the 7-amino group to the pyrone ring in the ground state. The emission maxima of 3-fluorocoumarins nearly coincide with the fluorescence maxima of the corresponding 3-chlorocoumarins; and this means that 3-fluorocoumarins have larger Stokes shifts $(92-113 \mathrm{~nm})$. Fluorescence QYs of 7-amino-3- 
fluorocoumarins in EtOH are high. However, the introduction of a fluorine atom into the position 6 in compounds $\mathbf{9 9}$ and $\mathbf{1 0 0}$ results in a significant decrease of emission efficiency.

The main absorption maximum of 7-amino-3-formylcoumarins (101-107 and 144-146) is shifted by 30-70 $\mathrm{nm}$ towards the red region of the visible spectrum compared with the analogous 3-unsubstituted 7-aminocoumarins. ${ }^{[40]}$ All coumarins in this series, except 144 and 145 with the julolidine fragment, exhibit weak fluorescence in $\mathrm{EtOH}$ and $\mathrm{MeCN}\left(\Phi_{\mathrm{fl}}\right.$ $<0.10$ ). Decrease in the emission efficiency in case of coumarins with a rotating 7-amino group (101-107) can be attributed to the formation of TICT state which is stabilized by the strongly electron-withdrawing formyl group. The observed Stokes shifts were relatively small (41-54 nm) for compounds 101-103, 106 and 144-146 with "small" substituents at $\mathrm{C}-4(\mathrm{H}, \mathrm{Me}$ and $\mathrm{Cl})$. On the other hand, bulky substituents, such as NHBn and $N$-morpholyl in coumarins 104, 105 and 107 provided larger Stokes shifts (66-88 nm) which are more common for coumarin dyes.

Introduction of $\mathrm{N}$-imidazolyl and $\mathrm{N}$-benzimidazolyl fragments into the position 4 of the coumarin skeleton (compounds 108, 109 and 147) is accompanied by bathochromic $(14-21 \mathrm{~nm})$ and bathofluoric shifts $(14-20 \mathrm{~nm})$ in absorption and emission spectra in EtOH, respectively (as compared with 4-unsubstituted analogs). ${ }^{[41]}$ Additionally, the presence of $N$-heteroaryl residues leads to a significant decrease in the fluorescence QYs, which is most pronounced in the case of 7- $N, N$-diethylaminocoumarins 108 and 109. The separation of the heteroaromatic fragments by a methylene group in compounds 110-114, 148 and 149 is accompanied by a hypsochromic shift of 10-15 nm compared with compounds without the methylene group $(66,68,69,108,109,147$ and 150) and has virtually no effect on the location of the fluorescence maximum. The transition from coumarins 108, 109 and 147 to coumarins 113, 114 and 149 is also accompanied by an increase in emission intensities.

In the absorption spectra of compounds 118-128, the position of the long-wavelength absorption band depends relatively weakly on the nature of the substituent on the phenyl ring. ${ }^{[42]}$ There is only a small $(<10 \mathrm{~nm})$ bathochromic shift of this spectral band in the transition from the electron-donating $(\mathrm{Me}, \mathrm{OPh})$ to electron-withdrawing groups $(\mathrm{F}, \mathrm{Cl}$, $\mathrm{CN}$ ) in the ortho- or para-position. This indicates the presence of only a weak $\pi-\pi$ conjugation between the aromatic substituent and the coumarin core. Another evidence for the 
weak electronic interactions between 3-aryl groups and the rest of the fluorophore is illustrated by the similarity of the spectral properties of 3-phenyl derivatives $\mathbf{1 1 8}$ and $\mathbf{1 1 9}$ with those of the related 7-N,N-diethylaminocoumarin 133 and 7- $N, N$-dimethylamino-4methylaminocoumarin $\mathbf{4 4}$ which have no aryl group at C-3. The position of the absorption maximum is affected more substantially by the nature of the 4-substituent. The increase in electron-withdrawing properties of the substituent series $\mathrm{N}$-morpholyl $-\mathrm{Me}-\mathrm{Cl}-\mathrm{H}-$ $\mathrm{CF}_{3}$ at $\mathrm{C}-4$ is accompanied by a red-shift of the main absorption maximum of more than $30 \mathrm{~nm}$. The fluorescence spectra are significantly more sensitive to the effects of the substituents. Thus, in EtOH, compounds 118-131 emit in the region of 460-545 nm. Compounds 118-128 exhibit particularly strong fluorescence. For coumarins 123-128, the bathofluoric shift of the fluorescence maximum in the transition from the ortho- to the para-substituted derivatives amounts to $10-15 \mathrm{~nm}$. In 7-N,N-diethylamino-3-( $\beta$ styryl)coumarin 132, the presence of the trans-configured double bond leads to a bathochromic shift of $24 \mathrm{~nm}$ and has virtually no influence on the position of the fluorescence maxima and the value of the fluorescence $\mathrm{QY}^{[43]}$ as compared to 7-diethylamino-3phenylcoumarin 118.

Takechi et al. ${ }^{[44]}$ prepared 3-azolyl-7-N,N-diethylaminocoumarins 152-179 (Table 7) and studied the influence of the heterocycle nature and its substitution pattern on spectral properties. Thus, in $\mathrm{EtOH}$, all the compounds exhibited red-shifts of the absorption (10-60 nm) and emission (3-35 nm) maxima combined with an increase in molar absorptivities (up to 1.7 times) compared to reference compound 7- $N, N$-diethylamino-3-phenylcoumarin $118\left(\lambda_{\mathrm{abs}, \max }=398 \mathrm{~nm}, \lambda_{\text {flu,max }}=477 \mathrm{~nm}\right)$. The relative fluorescence intensity (RFI) turned out to be similar or even higher (up to 1.93 times) than that of the reference coumarin 118 (RFI = 1.00), except for 3-(1,3-thiazol-4-yl)- and 3-(1,3,4-oxadiazol-2-yl)substituted compounds 172-175. According to the magnitude of bathochromic and hyperchromic ${ }^{*}$ shifts, heteroaromatic substituents can be arranged in the following order: 1,3,4thiadiazol-2-yl $(48 \mathrm{~nm})>$ 1,3-thiazol-2-yl $(39 \mathrm{~nm})>$ 1,3,4-oxadiazol-2-yl $(31 \mathrm{~nm})>$ 1,3oxazol-2-yl (26 nm). Values of the fluorescence maximum and the RFI exhibit a tendency to decrease in the order 1,3-thiazol-2-yl (495 nm, 1.42) 21,3,4-thiadiazol-2-yl (494 nm, $1.01)>1,3$-oxazol-2-yl (481 nm, 1.02) $\geq 1,3,4$-oxadiazol-2-yl (480 nm, 0.35). The values of $\lambda_{\text {flu,max }}$ of coumarins with diazole substituents are similar to those of coumarins with

\footnotetext{
${ }^{*}$ Hyperchromic shift is an increase in the absorbance.
} 
azole residues, but the presence of an additional nitrogen substantially decreases the emission intensity. The influence of 1,3-thiazolyl and 1,3,4-thiadiazolyl groups on the spectral properties is larger than the influence of the corresponding 1,3-oxazolyl and 1,3,4-oxadiazolyl groups. Authors ${ }^{[44]}$ suggested the sulfur lone pair in thiazoles is more delocalized than that of the oxygen in oxazole giving rise to a more effective $\pi-\pi$ conjugation between the thiazole substituent and the coumarin ring. Furthermore, the low-lying $d$ orbitals of the sulfur may also play an additional role in the conjugation. As a result, thiazole-containing compounds absorb and emit at longer wavelengths than the corresponding oxazole analogs.

For regioisomeric 3-(phenyl-1,3-oxazolyl)coumarins 157, 158, 161 and 162 (see Table 7) the values of the $\lambda_{\text {abs,max }}, \lambda_{\text {flu,max }}$ and $\varepsilon$ decrease in order: 5-phenyl-1,3-oxazol-2-yl (437 nm, $498 \mathrm{~nm}, 47300 \mathrm{M}^{-1} \mathrm{~cm}^{-1}$, respectively) > 2-phenyl-1,3-oxazol-5-yl (433 nm, $492 \mathrm{~nm}$, $\left.46900 \mathrm{M}^{-1} \mathrm{~cm}^{-1}\right)>4$-phenyl-1,3-oxazol-2-yl (428 nm, $488 \mathrm{~nm}, 47300 \mathrm{M}^{-1} \mathrm{~cm}^{-1}$ ) > 2-phenyl-1,3-oxazol-4-yl (411 nm, $476 \mathrm{~nm}, 38800 \mathrm{M}^{-1} \mathrm{~cm}^{-1}$ ). The RFI decreased in the order: 2-phenyl-1,3-oxazol-5-yl (1.43) > 5-phenyl-1,3-oxazol-2-yl (1.32) > 4-phenyl-1,3-oxazol2-yl (1.21) > 2-phenyl-1,3-oxazol-4-yl (1.15). The similar trends were observed for the corresponding sulfur analogs.

Variation of substituents in position 4 and 5 of the thiazole ring in 3-(1,3-thiazol-2yl)coumarins 163-167, 169 and 170 revealed that the values of $\lambda_{\text {abs,max }}, \lambda_{\text {flu,max }}$ and $\varepsilon$ decreased in the order COOEt $>\mathrm{Ph}>\mathrm{Me}>\mathrm{H}$ in both 4- and 5-thiazole-substituted derivatives. Thus, an ethoxycarbonyl group at C-4 and C-5 of the thiazole has the largest influence on the absorption and fluorescence properties, whereas a methyl group has almost no or only a little effect. Moreover, the influence of an ethoxycarbonyl or a phenyl substituent at C-5 was greater than the effect of the same substituent at C-4 (due to the better push-pull effect between the 7-amino group of the coumarin and the electron-withdrawing group of the azole). 
Table 7 Spectral properties of 7- $N, N$-diethylaminocoumarins with azole substituents at C-3 in EtOH.

\begin{tabular}{|c|c|c|c|c|c|c|}
\hline \multicolumn{2}{|r|}{ Compound } & $\lambda_{\text {abs,max }}, \mathrm{nm}$ & $\varepsilon, \mathrm{M}^{-1} \mathrm{~cm}^{-1}$ & $\lambda_{\mathrm{em}, \max }, \mathrm{nm}$ & $\Delta \lambda$, & RFI \\
\hline 152 & & 424 & 41100 & 481 & 57 & 1.02 \\
\hline 153 & & 425 & 42800 & 484 & 59 & 1.15 \\
\hline 154 & & 430 & 45200 & 483 & 53 & 0.96 \\
\hline 155 & & 443 & 48700 & 494 & 51 & 1.23 \\
\hline 156 & & 442 & 49700 & 493 & 51 & 1.49 \\
\hline 157 & & 428 & 43100 & 488 & 60 & 1.21 \\
\hline 158 & & 437 & 47300 & 498 & 61 & 1.32 \\
\hline 159 & & 422 & 40500 & 480 & 58 & 1.25 \\
\hline 160 & & 447 & 47900 & 504 & 57 & 1.45 \\
\hline 161 & & 433 & 46900 & 492 & 59 & 1.43 \\
\hline
\end{tabular}




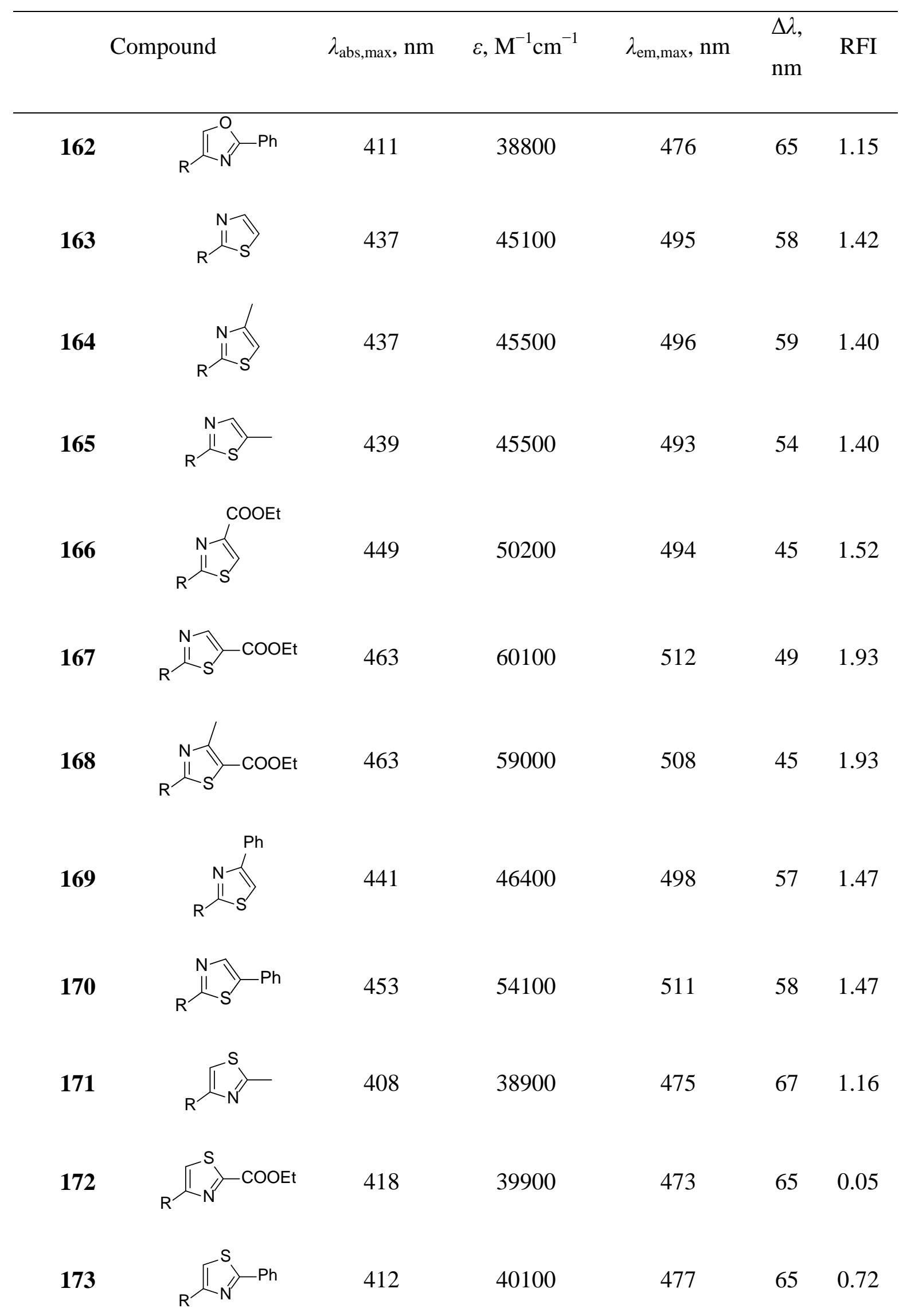




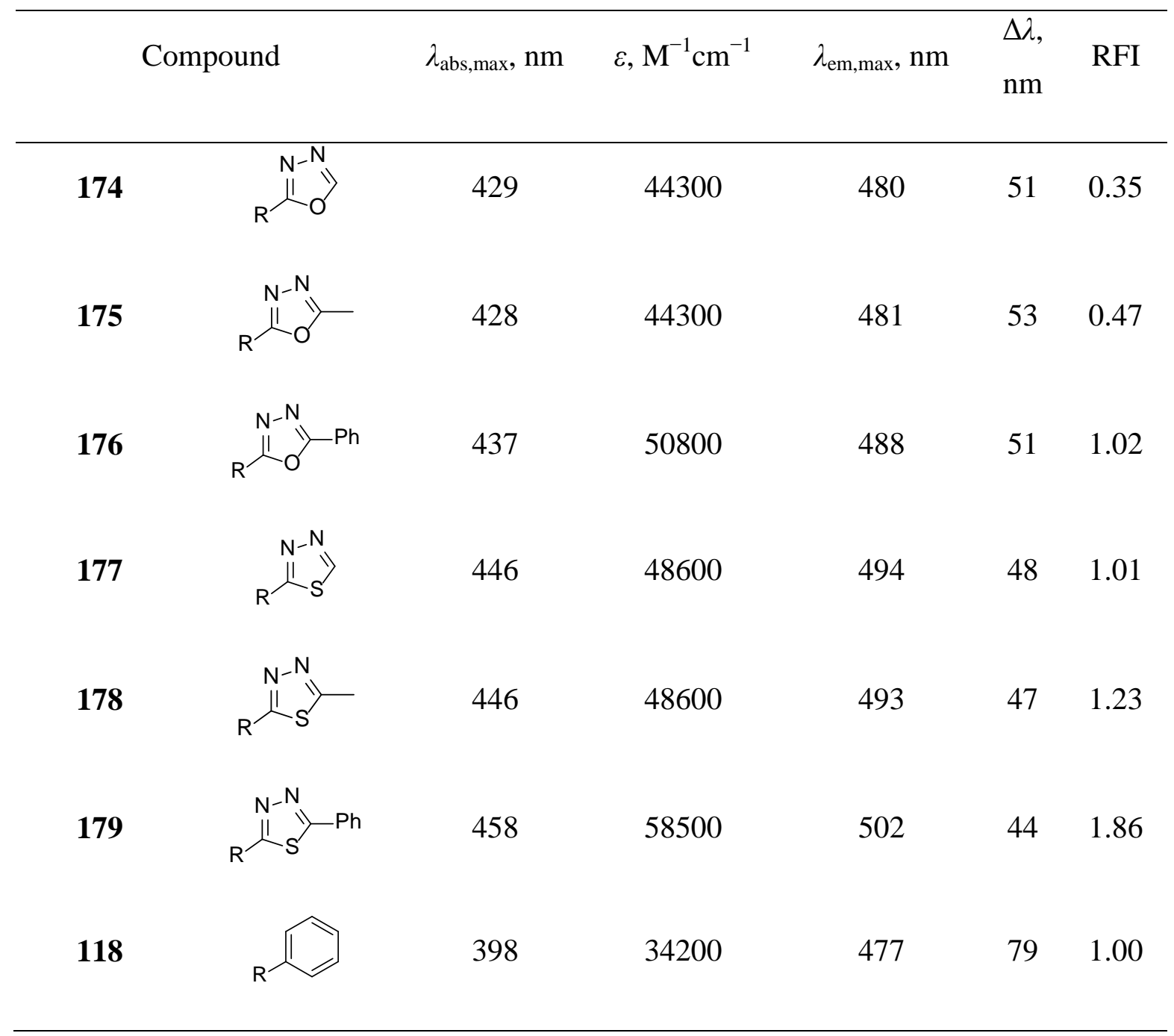

Regarding the synthetic routes leading to various 3-substituted coumarins, we conclude that many of them rely on the modern Pd-catalyzed C-C cross-couplings such as Suzuki, Heck and Sonogashira reactions applied to 3-halocoumarins. ${ }^{[45]}$ These transformations allow the introduction of arene, ethynylene and ethenylene moieties to the coumarin skeleton in one synthetic step. Taking advantage of these reactions and utilizing a combinatorial synthetic approach, Schiedel et al. ${ }^{[46]}$ prepared a compound library consisting of 34 coumarins with various substitution patterns. The "hits" (best dyes) were selected on the basis of fluorescence QYs and the possibility of further modifications (for applications as fluorescent labels). Thus, compound 180 (Figure 14) combines a very large Stokes shift of $162 \mathrm{~nm}\left(\lambda_{\mathrm{abs}, \max } / \lambda_{\mathrm{flu} \text {,max }}=373 / 535 \mathrm{~nm}\right.$, in EtOH$)$ with a moderate quantum yield $\left(\Phi_{\mathrm{fl}}=0.18\right)$, compounds $181(395 / 478 \mathrm{~nm}), 182(393 / 480 \mathrm{~nm})$ and $183(397 / 455$ $\mathrm{nm}$ ) have the highest emission efficiencies of 0.90, 0.62 and 0.98 in EtOH, respectively. Remarkably, compound 183 exhibited a higher fluorescence QY than the similar and 
commercially available Coumarin 120 (41) (354/435 nm, $\Phi_{\mathrm{fl}}=0.88$, in EtOH). The 7$\mathrm{NH}_{2}$-substituted compounds $\mathbf{1 8 0}, \mathbf{1 8 2}$ and $\mathbf{1 8 3}$ can be linked to the biomolecule of interest through their modified amino groups.<smiles>Cc1c(-c2cccc3ccccc23)c(=O)oc2ccc(N)cc12</smiles>
180<smiles>Cc1cc(=O)oc2cc(N)ccc12</smiles>

41 (Coumarin 120)<smiles>Cc1ccc(/C=C/c2c(C)c3ccc(N)cc3oc2=O)cc1</smiles>

181
182

Figure 14 Coumarins prepared by a combinatorial method from 3-halocoumarins.

Another dye library was obtained by a "click” reaction of 3-azidocoumarins with various substituted alkynes. ${ }^{[47]} 7$-Aminosubstituted coumarins exhibited strong fluorescence in EtOH (QYs 0.6-0.7) and a rather small dependence on the nature of the substituent attached to C-4 of the triazolyl ring. Thus, all 24 investigated 7-diethylaminocoumarins 184-R (see Figure 15) have absorption and emission maxima in the narrow intervals 413-418 $\mathrm{nm}$ and 484-489 $\mathrm{nm}$ in aq. DMSO, respectively. Analogously, the absorption and emission bands of 7-aminocoumarins 185-R with the julolidine fragment are grouped around 431-436 $\mathrm{nm}$ and 503-509 $\mathrm{nm}$ in aq. EtOH, respectively. 


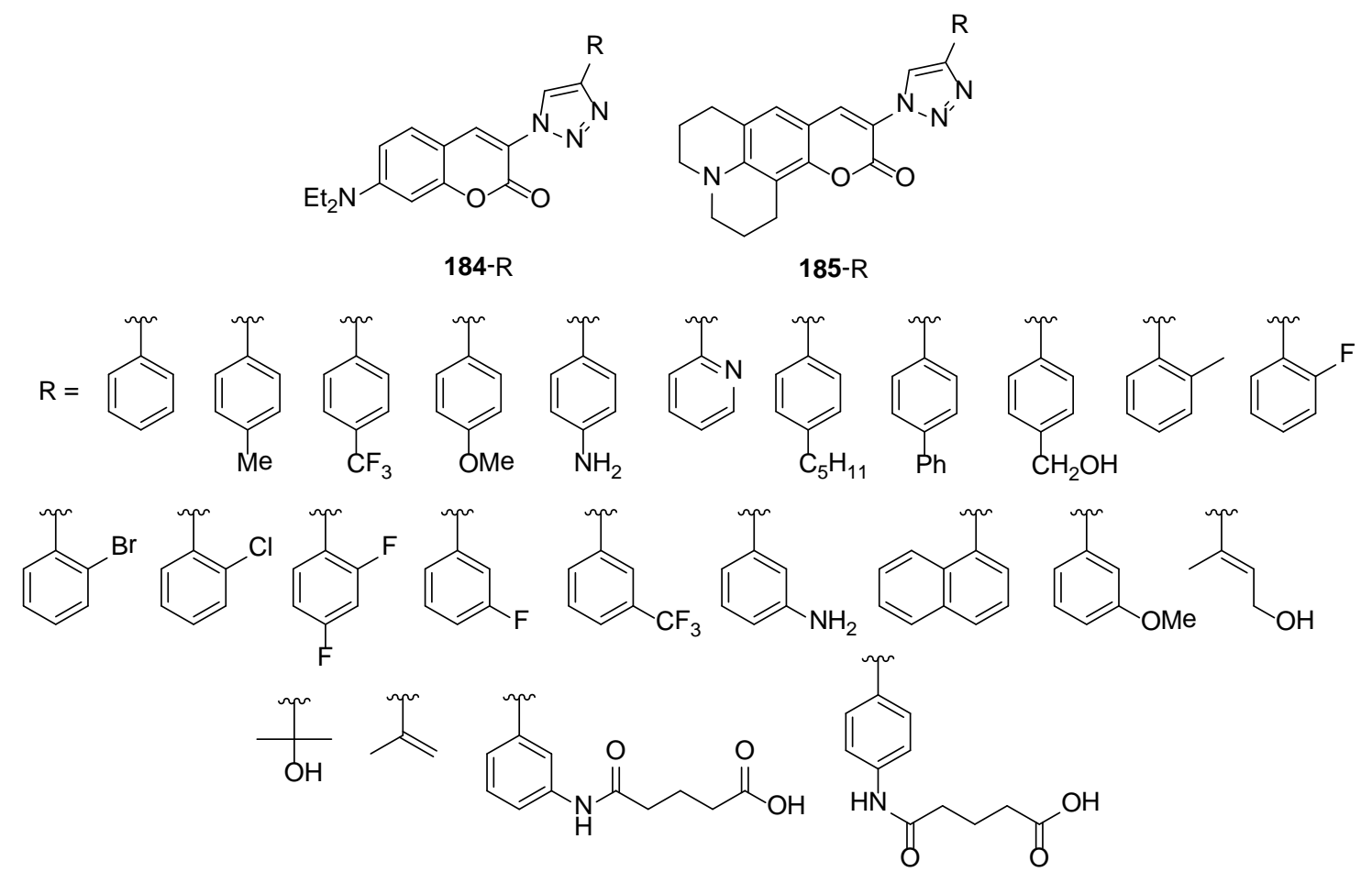

Figure 15 7-Amino-3-(1,2,3-triazol-1-yl)coumarins prepared by a combinatorial method.

Another series of coumarin dyes (Figure 16) was studied in order to evaluate the influence of various nitrogen-containing groups in position 7 on the optical spectra. ${ }^{[48]}$ The nitrogen atom incorporated into a piperidine fragment in compound $\mathbf{1 8 9}-\mathrm{H}$ has a somewhat greater electron-releasing ability in comparison to the pyrrolidine-fused coumarin 188-H. In ethanolic solutions, this results in a small bathochromic $(8 \mathrm{~nm})$ and a bathofluoric $(4 \mathrm{~nm})$ shift in the transition from compound $188-\mathrm{H}\left(\lambda_{\mathrm{abs} \text {,max }} / \lambda_{\mathrm{flu} \text {,max }}=\right.$ $370 / 440 \mathrm{~nm})$ to $\mathbf{1 8 9}-\mathrm{H}(378 / 444 \mathrm{~nm})$. The highest occupied molecular orbital (HOMO) of pyrrole has a node at the nitrogen atom. Therefore, the connection of an $N$-pyrrolyl moiety at C-7 of the coumarin diminishes $\pi-\pi$ conjugation between the pyrrole and coumarin systems and decreases the extent of the ICT in the excited state of compounds 186$\mathrm{R}$ and $\mathbf{1 8 7}-\mathrm{H}$ in comparison to 7-aminocoumarins. As a result, for compounds $186-\mathrm{H}$ $\left(\lambda_{\mathrm{abs}, \max }=329 \mathrm{~nm}\right), \mathbf{1 8 6}-\mathrm{F}\left(\lambda_{\mathrm{abs}, \max }=342 \mathrm{~nm}\right)$ and $187-\mathrm{H}\left(\lambda_{\mathrm{abs}, \max }=316 \mathrm{~nm}\right)$ relatively large hypsochromic shifts $(24-40 \mathrm{~nm})$ were observed, relative to the corresponding 7amino-3-methyl- or 7-amino-3-trifluoromethylcoumarin (compounds 41 and 39, respectively). Carbazole-coumarin hybrids 190-R and 191-R absorb in the near-UV region with a maximum at 353-376 $\mathrm{nm}$ and emit in the blue and green regions of the visible spectra. Notably, compounds 191-F and 192-F with the 4-trifluoromethyl group provide the 
largest Stokes shifts of 163 and $124 \mathrm{~nm}$. However, their emission intensities in EtOH are very low (less than 0.02 ).<smiles>[R16]c1cc(=O)oc2cc(-n3cccc3)ccc12</smiles>

186-R<smiles>[R3]c1cc(=O)oc2cc3c(cc12)CCCN3</smiles>

189-R<smiles></smiles>

187-R

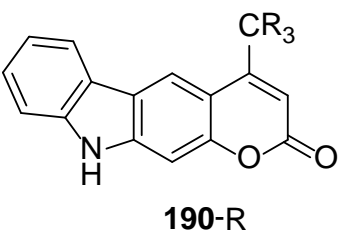

$\mathrm{R}=\mathrm{H}, \mathrm{F}$<smiles>[R3]c1cc(=O)oc2cc3c(cc12)CCN3</smiles>

188-R<smiles>[R3]c1cc(=O)oc2c1ccc1[nH]c3ccccc3c12</smiles>

191-R

Figure 16 Coumarins 186-R-191-R with various substitution patterns at nitrogen atom.

In the search for a novel fluorogenic indicator for monoamine oxidases (MAOs), Chen et al. ${ }^{[49]}$ prepared a number of fused pyrrolocoumarins (Figure 17) with various fusion patterns. In EtOH, compounds 192 and 193 with the nitrogen atom attached to C-7 turned out to be almost non-fluorescent. Compounds $194\left(\lambda_{\mathrm{abs}, \max } / \lambda_{\text {flu,max }}=335 / 524 \mathrm{~nm}\right)$ and 196 $(330 / 517 \mathrm{~nm})$ had very large Stokes shifts of 189 and $187 \mathrm{~nm}$, respectively, in the series. However, their QYs in polar solvents were very low (0.08 and 0.06 in EtOH). Coumarin $195(349 / 492 \mathrm{~nm})$ provided the best combination of a large Stokes shift $(143 \mathrm{~nm})$ and a moderate fluorescence QY (0.36 in EtOH) in this series of pyrrolocoumarins.<smiles>Cc1cc(=O)oc2cc3[nH]ccc3cc12</smiles>
192<smiles>Cc1cc(=O)oc2c1ccc1[nH]ccc12</smiles>
193<smiles>O=c1ccc2cc3[nH]ccc3cc2o1</smiles>

194<smiles>Cc1cc(=O)oc2ccc3[nH]ccc3c12</smiles>
195<smiles>O=c1ccc2c(ccc3cc[nH]c32)o1</smiles>

196

Figure 17 Pyranoindolones 192-196 as fused fluorophores with the coumarin core.

Fusion of 2-phenyl- and 2-trans-styrylimidazole systems with C-6 and C-7 positions of the coumarin scaffold (Figure 18) results in hypsochromic shifts of 30 and $7 \mathrm{~nm}$ as well as hypsofluoric shifts of 24 and $23 \mathrm{~nm}$, respectively, and in a two-fold decrease in fluorescence QY in $\mathrm{EtOH}^{[50]}$ when compared with 7- $N, N$-diethylamino-4-methylcoumarin $44\left(\lambda_{\mathrm{abs}, \max } / \lambda_{\mathrm{flu}, \max }=373 / 445 \mathrm{~nm}, \Phi_{\mathrm{fl}}=50 \%^{[21 \mathrm{a}]}\right)$. 


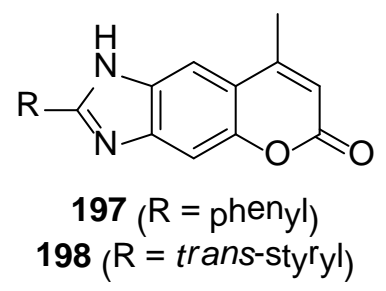

Figure 18 8-Methylchromeno[7,6- $d$ ]imidazol-6(1H)-ones $\mathbf{1 9 7 - 1 9 8}$ as fused fluorophores based on the coumarin core.

Coumarins 199-R-201-R have various substituents at C-3 and different secondary amino groups at C-7. Comparison of compounds with the same 3-substituent revealed that a transition from compounds 199-R to 201-R provides red-shifts of 17 and $9 \mathrm{~nm}$ in the absorption bands and small red-shifts of 5 and $13 \mathrm{~nm}$ in the emission bands, respectively. ${ }^{[45 b]}$ The substituents at C-3 have a stronger influence on the positions of the absorption and emission bands. Thus, compared to 3-unsubstituted coumarin 201- $\mathrm{H}$ $\left(\lambda_{\text {abs,max }} / \lambda_{\text {flu,max }}=424 / 527 \mathrm{~nm}\right.$, in EtOH) the bathochromic shifts increased in the following order of substituents at C-3: 2-pyridyl $(14 \mathrm{~nm}) \leq 4$-pyridyl $(16 \mathrm{~nm})<2$-thienyl $(21 \mathrm{~nm})<$ trans-2-phenylethenyl (43 nm) $<N$-(3-sulfopropyl)-4-pyridinio $(50 \mathrm{~nm}) \leq$ trans-2-pyridylethenyl (51 nm) $<N$-(3-sulfopropyl)-4-pyridinio (55 nm) $\leq$ trans-4-pyridylethenyl (56 $\mathrm{nm})$. Similar trends were observed in compounds 199-R and 200-R. For compounds 201$\mathrm{R}$ two new substituents were included, namely, $N$-(5-carboxypentyl)-2-pyridinioethenyl and $N$-(5-carboxypentyl)-4-pyridinioethenyl. These groups produce further red-shifts of $31 \mathrm{~nm}$ and $51 \mathrm{~nm}$, respectively, in comparison to their non-quaternized analogs. For the emission bands, the bathofluoric shifts increase in a similar order. Exceptions are the 2thienyl and 4-[N-(3-sulfopropyl)pyridinium] groups, which shift the emission bands very strongly to the red spectral region and show very large Stokes shifts of around 200 and $230 \mathrm{~nm}$, respectively. In the former case, this effect, according to the authors, ${ }^{[45 \mathrm{~b}]}$ is probably due to the strong electron-donating property of the 2-thienyl group. The fact that 4-[N-(3-sulfopropyl)pyridinium] group provides a much larger Stokes shift $(230 \mathrm{~nm})$ than the structurally similar 2-[N-(3-sulfopropyl)pyridinium] group $(145-150 \mathrm{~nm})$ was explained by a larger dipole moment. Similarly, 4-pyridyl substituents shift the emission bands more to the red compared with 2-pyridyl groups. Unfortunately, dyes with huge Stokes shifts exhibited low fluorescence QYs in EtOH. 

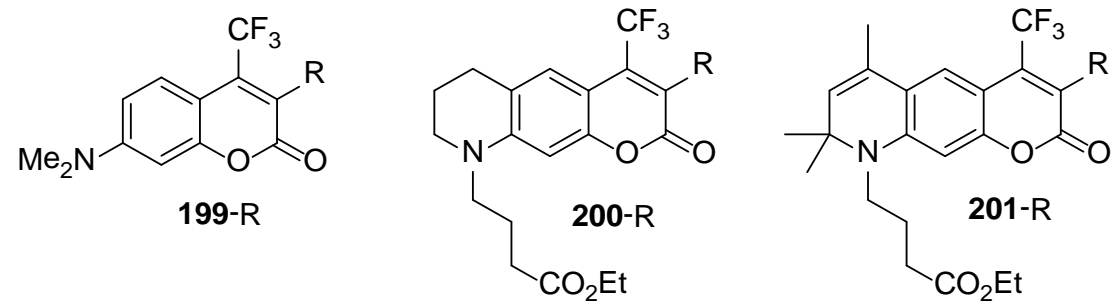

Table 8 Spectral properties of 4-trifluoromethylcoumarins 199-R-201-R in ethanolic solutions.

\begin{tabular}{|c|c|c|c|c|c|c|}
\hline Compound & $\mathrm{R}$ & $\lambda_{\text {abs,max }}, \mathrm{nm}$ & $\varepsilon, \mathrm{M}^{-1} \mathrm{~cm}^{-1}$ & $\lambda_{\text {em,max }}, \mathrm{nm}$ & $\Delta \lambda, \mathrm{nm}$ & $\Phi_{\mathrm{fl}}$ \\
\hline \multirow{8}{*}{ 199-R } & 2-thienyl & 416 & 24400 & 611 & 195 & 0.13 \\
\hline & $\mathrm{CH}=\mathrm{CH}-2-\mathrm{py}$ & 444 & 36600 & 578 & 134 & 0.64 \\
\hline & $\mathrm{CH}=\mathrm{CH}-2-\mathrm{py}-\mathrm{A}^{\mathrm{a}}$ & 475 & 9900 & 646 & 171 & 0.37 \\
\hline & $\mathrm{CH}=\mathrm{CH}-4-\mathrm{py}$ & 449 & 28500 & 580 & 131 & 0.71 \\
\hline & $\mathrm{CH}=\mathrm{CH}-4-\mathrm{py}-\mathrm{A}$ & 500 & 13300 & 668 & 168 & 0.47 \\
\hline & 4-py & 413 & 18800 & 588 & 175 & 0.47 \\
\hline & 4-ру-А & 439 & 11800 & 644 & 205 & 0.27 \\
\hline & $\mathrm{CH}=\mathrm{CH}-\mathrm{ph}$ & 438 & 27600 & 576 & 138 & 0.49 \\
\hline \multirow{4}{*}{ 200-R } & 4-py & 430 & 13700 & 593 & 163 & 0.19 \\
\hline & 4-py-B ${ }^{\mathrm{b}}$ & 459 & 14800 & 655 & 196 & 0.34 \\
\hline & 2-py & 429 & 16400 & 578 & 149 & 0.20 \\
\hline & 2-ру-В & 462 & 19200 & 607 & 142 & 0.11 \\
\hline \multirow{5}{*}{ 201-R } & 4-py & 440 & 18100 & 611 & 171 & - \\
\hline & 2-py & 438 & 17900 & 591 & 153 & 0.19 \\
\hline & 2-ру-В & 479 & 22400 & 632 & 153 & 0.09 \\
\hline & 4-ру-В & 474 & 12200 & 687 & 213 & 0.12 \\
\hline & 2-thienyl & 445 & 17400 & 636 & 191 & 0.16 \\
\hline
\end{tabular}




\begin{tabular}{ccccccc}
\hline Compound & $\mathrm{R}$ & $\lambda_{\text {abs,max }}, \mathrm{nm}$ & $\varepsilon, \mathrm{M}^{-1} \mathrm{~cm}^{-1}$ & $\lambda_{\text {em,max }}, \mathrm{nm}$ & $\Delta \lambda, \mathrm{nm}$ & $\Phi_{\mathrm{fl}}$ \\
\hline $\mathrm{CH}=\mathrm{CH}-\mathrm{ph}$ & 467 & 20000 & 598 & 131 & 0.58 \\
$\mathrm{CH}=\mathrm{CH}-2-\mathrm{py}$ & 475 & 30400 & 602 & 127 & 0.80 \\
$\mathrm{CH}=\mathrm{CH}-4-\mathrm{py}$ & 480 & 32100 & 609 & 129 & 0.68 \\
$\mathrm{H}$ & 424 & 19600 & 527 & 103 & 0.45
\end{tabular}

\footnotetext{
${ }^{a} \mathrm{~A}$ : The pyridine nitrogen is alkylated with an $\omega$-carboxypentyl residue. ${ }^{b} \mathrm{~B}$ : The pyridine ring is alkylated with an $\omega$-sulfopropyl group.
}

In benzocoumarins (2H-benzochromen-2-ones) the annelation mode affects both the absorption and fluorescence properties. The values of absorption maxima for benzocoumarin derivatives in MeCN decrease in the order 203b $\left(\lambda_{\mathrm{abs}, \max } / \lambda_{\mathrm{flu} \text {,max }}=390 / 491 \mathrm{~nm}\right.$, $\left.8600 \mathrm{M}^{-1} \mathrm{~cm}^{-1}\right)>202 \mathbf{b}\left(382 / 465 \mathrm{~nm}, 15100 \mathrm{M}^{-1} \mathrm{~cm}^{-1}\right)>204 \mathbf{b}$ (337/549 nm, $22500 \mathrm{M}^{-1} \mathrm{~cm}^{-1}$ ) with increasing absorbance (for structures, see Figure 19). ${ }^{[51]}$ The same tendency was observed for 3-ethoxycarbonylcompounds (203a > 202a > 204a). This was explained by the increase in extent of the CT between the benzocoumarin skeleton and the substituent at C-3. Among these benzocoumarins, compounds 202a (ester type) and 203b (acetyl type) showed the highest fluorescence efficiency. In contrast to the absorption maxima, the emission maxima shifted to longer wavelengths in the order $204>203>$ 202. The "linear" compounds 204a (331/535 nm) and 6b (337/549 nm) showed remarkably large Stokes shifts of 204 and 212 nm, respectively. These values are twofold greater than those for "angular" compounds. However, the emission intensity of "linear" fused compounds is very poor and significantly inferior to that of the "angular" compounds.

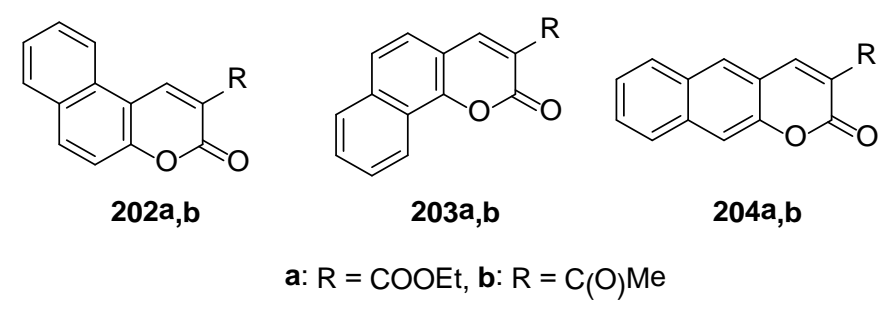

Figure 19 Benzocoumarins (2H-benzochromen-2-ones) 202-204 with various annelation modes.

In "iminocoumarins" the carbonyl oxygen in the position 2 is replaced with an imino group (see Figure 20). Sometimes these compounds exhibit photophysical properties 
which are superior to those of the "parent" coumarins. Furthermore, in contrast to simple coumarins, the imino group can be easily modified allowing the synthesis of various derivatives. For example, iminocoumarin 205 shows a red-shifted absorption maximum $\left(\lambda_{\text {abs,max }}=503 \mathrm{~nm}\right)$ in comparison with the "normal” coumarin $51\left(\lambda_{\mathrm{abs}, \max }=467 \mathrm{~nm}\right)$, a well-known laser dye Coumarin 545. ${ }^{[52]}$ Compound 205 also has higher emission efficiency in pH-buffered aq. solutions (63\% vs 10\% for compound 51). However, the observed Stokes shift (34 nm) is usually small for coumarin compounds. The modification potential of compound 205 was efficiently used in the design of various indicators and sensors for $\mathrm{Zn}^{2+}$, ${ }^{[52]} \mathrm{Cu}^{2+[53]}$ and $\mathrm{F}^{-[54]}$ and for the detection of alkaline phosphatase activity in living cells. ${ }^{[55]}$ Another iminocoumarin 206 contains a tetrahydroquinoxaline moiety and, when dissolved in neutral aq. buffer, with $143 \mathrm{~nm}$ exhibits a significantly larger Stokes shift $\left(\lambda_{\mathrm{abs}, \max } / \lambda_{\mathrm{flu} \text {,max }}=473 \mathrm{~nm} / 616 \mathrm{~nm}\right)$ than the dye 205. Such a large Stokes shift was beneficial for signal detection in sensing fluoride ions. ${ }^{[56]}$
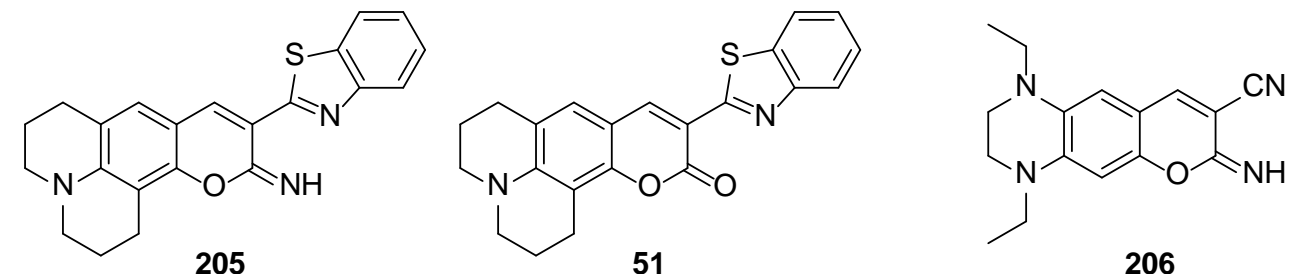

Figure 20 Iminocoumarins 205-206 and Coumarin 545 (51).

1,4-2H-Benzoxazin-2-ones, also known as “azacoumarins”, are coumarin analogs in which the carbon atom C-4 is replaced with a nitrogen (Figure 21). Similarly to the corresponding 7-aminocoumarins, 7-aminoazacoumarins 207-R display strong fluorescence, but it is shifted towards the red spectral region due to a bathochromic effect of the heterocyclic nitrogen. This effect resembles the case of oxazine dyes (nitrogen and oxygen atoms at C-9 and C-10, respectively) which absorb and emit at longer wavelengths than the corresponding rhodamines (carbon and oxygen at C-9 and C-10, respectively). The spectral properties of compounds 207- $\mathrm{R}$ in various solvents were thoroughly investigated. ${ }^{[57]}$ It was shown that the observed Stokes shift can reach an unusually high value of $183 \mathrm{~nm}$, while the parent coumarin dyes showed Stokes shifts of $100 \mathrm{~nm}$ or less (in the same range of solvent polarity). A high fluorescence quantum yield of $93 \%$ in $\mathrm{CHCl}_{3}$ was reported for compound 207-Me. Large Stokes shifts of azacoumarins allowed using 7$N, N$-dimethylamino-3-(p-formylstyryl)-1,4-benzoxazin-2-one 208 and benzoxazinone 
vinylcinnamic acid $\mathbf{2 0 9}$ as fluorescent dyes in two-color cytometry together with fluorescein derivatives (which possess small Stokes shifts) using only one excitation wavelength. ${ }^{[58]}$ Although the emission efficiencies in polar protic media were reported only for a limited number of compounds, azacoumarins are interesting as scaffolds for the design of better large Stokes shift dyes which can be used in fluorescence microscopy.<smiles></smiles>
$\mathrm{R}=\mathrm{Me}, \mathrm{COOEt}, \mathrm{CONH}_{2}, \mathrm{CONHPh}, \mathrm{CN}$,<smiles>CC=C(C(=O)OCC)C(=O)OCC</smiles><smiles>CCOC(=O)C(CC(C)(C)OC)(OCC)OOC</smiles><smiles>CCc1ccc(OC)cc1</smiles><smiles>CC(=O)c1cccc2ccccc12</smiles><smiles>CCOC(=O)/C(C)=C\CC/C=C(\OCC)c1ccccc1</smiles><smiles>Cc1ccc(N(C)C)cc1</smiles><smiles>CCC=Cc1ccc(N(C)C)cc1</smiles><smiles>CCC=Cc1ccc([N+](=O)[O-])cc1</smiles><smiles>CN(C)c1ccc2nc(/C=C/c3ccc(C=O)cc3)c(=O)oc2c1</smiles>

208<smiles>COc1ccc2nc(/C=C/c3ccc(/C=C/C(=O)O)cc3)c(=O)oc2c1</smiles>

209

Figure 21 7-Aminoazacoumarins 207-R, 208 and 209.

Another way to provide bathochromic and bathofluoric shift is the extension of the $\pi$ conjugated system. In this respect, 8- $N, N$-dimethylamino-2-oxo-2H-benzo[h]chro-menes $\mathbf{2 1 0}$ and 211 represent coumarins in which the $\pi$-conjgated system is extended by an additional benzene ring fused with positions 7 and 8 leading to a phenanthrene-like structure (Figure 22).<smiles>[R7]OC(=O)c1cc2ccc3cc(N(C)C)ccc3c2oc1=O</smiles><smiles>CCOC(=O)c1cc2ccc(N(C)C)cc2oc1=O</smiles>

43

Figure 22 8- $N, N$-Dimethylamino-2H-benzo[h]chromen-2-ones 210 and 211, and the parent coumarin 43. 
When dissolved in EtOH, compound 211 displays absorption and emission spectra maxima at 455 and $552 \mathrm{~nm}^{[59]}$ which corresponds to red-shifts of 43 and $92 \mathrm{~nm}$, respectively, compared with the parent dye 43, and a larger Stokes shift of $97 \mathrm{~nm} .{ }^{[21 \mathrm{a}]}$ A relatively high fluorescence quantum yield of $29 \%$ in aq. media (in the presence of sodium dodecyl sulfate), high photostability and a bathochromic shift upon binding with magnesium ions rendered compound $\mathbf{2 1 0}$ useful as a probe for selective detection of intracellular magnesium ions in living cells without interference by calcium ions, and even as a candidate for the two-photon excitation mode.

$2 \mathrm{H}$-Benzo[g]chromenes 212 and 214 represent analogues of 7- $N, N$-dimethylamino-coumarin 213 with an additional benzene ring fused to positions 6 and 7 of the coumarin core (Figure 23). ${ }^{[60]}$ Due to their extended $\pi$-system, benzocoumarin 212 and benzoiminocoumarin 214 absorb and emit at much longer wavelengths relative to the corresponding parent coumarin $213\left(\Delta \lambda_{\text {abs }}: 41\right.$ and $55 \mathrm{~nm} ; \Delta \lambda_{\mathrm{em}}: 110$ and $137 \mathrm{~nm}$ ) with larger quantum yields (61\% and 67\% in aqueous media, respectively). Emission bands are more shifted to the red than absorption, and this results in larger Stokes shifts of 152 and $139 \mathrm{~nm}$ compared with only $70 \mathrm{~nm}$ for compound 213. Recently, ${ }^{[61]}$ benzocoumarin 215 was proposed as a red-emissive and photostable two-photon fluorescence probe for mitochondria. This compound showed high selectivity and robust staining ability together with low cytotoxicity and insensitivity to $\mathrm{pH}$ changes in the biologically relevant $\mathrm{pH}$ range.

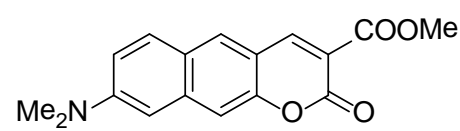

212

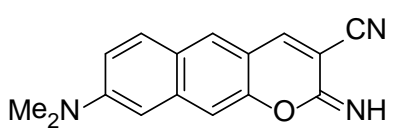

214<smiles>CC(=O)c1cc2ccc(N(C)C)cc2oc1=O</smiles>

213

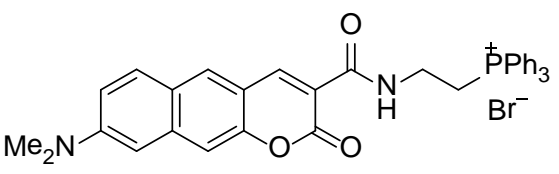

215

Figure $232 H$-Benzo[g]chromenes 212 and 214, two-photon fluorescent probe 215 and the parent coumarin fluorophore 213.

Here we abandon coumarin dyes and discuss the extension of the conjugated $\pi$-system in xanthene dyes. This approach could be applied to fluorescein, rhodol and rhodamine fluorophores. Fluorescent dyes with a red-shifted absorption band (compared with fluo- 
rescein) - naphthofluoresceins 216-R and 217-R (Figure 24) - were used in flow cytometry. ${ }^{[62]}$ Although the annelation mode used in compounds 216-R shifted absorption maximum by $\sim 30-40 \mathrm{~nm}$ relative to fluorescein, it did not result in a significant increase of Stokes shift which remained small (max $32 \mathrm{~nm}$ ). On the other hand, benzo[c]xanthene dyes 217-R displayed a red-shifted absorption with maxima at 595-609 nm (in aqueous media) and much larger Stokes shifts of 56-77 nm. However, their fluorescence QYs did not exceed $15 \%$.<smiles>[R]c1ccccc1-c1c2c3ccccc3c(=O)cc-2oc2cc(O)c3ccccc3c12</smiles>

216-R<smiles>[R]c1ccccc1-c1c2ccc3cc(O)ccc3c2oc2c1ccc1cc(=O)ccc12</smiles>

217-R

$\mathrm{R}=\mathrm{COOH}, \mathrm{SO}_{3} \mathrm{H}$

Figure 24 Naphthofluoresceins 216-R and 217-R.

Other benzo[c]xanthene dyes - seminaphthofluoresceins (SNAFLs) 218-222 and seminaphthorhodafluors (SNARFs) 223-231 ${ }^{[63]}$ - have only one fused benzene ring (Figure 25). They can be excited in the 550-650 nm range. Their $\mathrm{pK}_{\mathrm{a}}$ values lie within the physiologically relevant $\mathrm{pH}$ range (between 7 and 8). They also have larger Stokes shifts (up to $85 \mathrm{~nm}$ ) and good fluorescence QYs in aqueous media (up to 50\%). Unlike the fluoresceins, they show not only two distinct absorption bands for the protonated and deprotonated forms, but also two emission bands (because the molecules do not dissociate in the excited state). Furthermore, the spectra have clearly defined isosbestic and isoemissive wavelengths (where absorption or emission is $\mathrm{pH}$ independent). As a result, these compounds found numerous applications in ratiometric and fluorometric $\mathrm{pH}$ measurements ${ }^{[64]}$ and became commercially available. Apart from $\mathrm{pH}$ measurement, a number of SNAFL- and SNARF-based derivatives were used for detecting various metal ions, ${ }^{[65]}$ nitric oxide, ${ }^{[66]}$ thiols, ${ }^{[67]}$ peroxide ${ }^{[68]}$ and as a FRET partner for cyanine dye Cy5. ${ }^{[69]}$ 
<smiles></smiles>

$218\left(R^{1}=R^{2}=R^{3}=H\right)$ $219\left(R^{1}=\mathrm{CO}_{2} \mathrm{H}, \mathrm{R}^{2}=\mathrm{R}^{3}=\mathrm{H}\right)$ $220\left(R^{1}=\mathrm{CO}_{2} \mathrm{H}, \mathrm{R}^{2}=\mathrm{Cl}, \mathrm{R}^{3}=\mathrm{H}\right)$

$221\left(\mathrm{R}^{1}=\mathrm{CO}_{2} \mathrm{H}, \mathrm{R}^{2}=\mathrm{Cl}, \mathrm{R}^{3}=\mathrm{Cl}\right.$ $222\left(\mathrm{R}^{1}=\mathrm{CO}_{2} \mathrm{H}, \mathrm{R}^{2}=\left(\mathrm{CH}_{2}\right)_{2} \mathrm{CO}_{2} \mathrm{H}, \mathrm{R}^{3}=\mathrm{H}\right)$<smiles>[R]c1cc2c(-c3c[R]([H])ccc3C(=O)O)c3ccc4cc(=O)ccc4c3oc2c([R])c1[R]</smiles>

$226\left(R=M e, R^{1}=H\right)$ $227\left(R=E t, R^{1}=H\right)$ $228\left(R=M e, R^{1}=\mathrm{COOH}\right)$ $229\left(R=E t, R^{1}=\mathrm{COOH}\right)$<smiles>[R]c1cc2c(-c3cc[R7](O)cc3C(=O)O)c3ccc4cc(O)ccc4c3oc-2c([R])c1=N</smiles>

$223\left(\mathrm{R}=\mathrm{H}, \mathrm{R}^{1}=\mathrm{CO}_{2} \mathrm{H}, \mathrm{R}^{2}=\mathrm{H}\right)$

$224\left(R=E t, R^{1}=H, R^{2}=M e\right)$ $225\left(R=E t, R^{1}=\mathrm{CO}_{2} \mathrm{H}, R^{2}=M e\right)$<smiles></smiles>

$230(\mathrm{R}=\mathrm{H})$
$231(\mathrm{R}=\mathrm{Cl})$

Figure 25 Seminaphthofluoresceins (SNAFLs) 218-222 and seminaphthorhodafluors (SNARFs) 223-231.

Semiempirical calculations showed that $[a]$ - and $[b]$-annelated benzoxanthenes should absorb and emit at significantly longer wavelengths than their [c]-annelated analogs. ${ }^{[70]}$ Based on these findings, Yang et al. ${ }^{[71]}$ developed and prepared seminaphthofluorones (SNAFRs) 232-237 (Figure 26). The annelation patterns and the position of the hydroxyl group were varied, and the spectral properties of the derivatives were evaluated. It was found that both these variables have a relatively small effect on the spectral properties of the neutral forms of SNAFRs in DMSO: all compounds have absorption maxima near $475 \mathrm{~nm}$, and emission maxima near $600 \mathrm{~nm}$. In contrast to neutral forms, the spectral properties of deprotonated SNAFRs depend on the annelation type and the position of hydroxyl group. Thus, compound 236 in its anionic form shows a NIR emission with a maximum at $789 \mathrm{~nm}$, followed by 232 at $768 \mathrm{~nm}, 237$ at $725 \mathrm{~nm}$ and 235 at $694 \mathrm{~nm}$ (in DMSO). Benzo[c]xanthene dye 233, which is similar to SNAFL compounds, shows emission at the shortest wavelength $(673 \mathrm{~nm})$. The linearly annelated compound 237 is particularly interesting: in contrast to other SNAFRs, its anionic form exhibits an excep- 
tionally large Stokes shift of $197 \mathrm{~nm}\left(\lambda_{\mathrm{abs}}=536 \mathrm{~nm}, \lambda_{\mathrm{em}}=733 \mathrm{~nm}\right)$ in aqueous phosphate buffer.



232<smiles>O=c1ccc2c(-c3ccccc3)c3cc(O)ccc3oc-2c1</smiles>

235

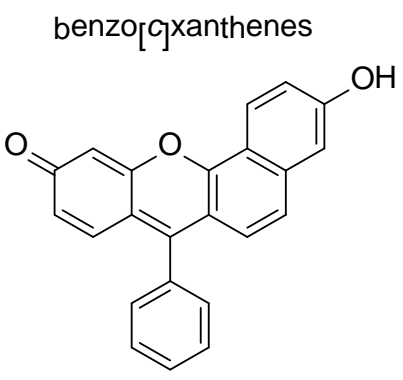

233<smiles>O=c1ccc2c(-c3ccccc3)c3ccc4cccc(O)c4c3oc-2c1</smiles>

236 benzo $[b]$ xanthenes<smiles>O=c1ccc2c(-c3ccccc3)c3cc4c(O)cccc4cc3oc-2c1</smiles>

234<smiles>O=c1ccc2c(-c3ccccc3)c3cc4ccc(O)cc4cc3oc-2c1</smiles>

237

Figure 26 Seminaphthofluorones (SNAFRs) 232-237.

A common feature of SNAFLs, SNARFs and SNAFRs is the sensitivity of their spectral properties to $\mathrm{pH}$ changes, but very often compounds with spectra insensitive to $\mathrm{pH}$ are needed. Rhodamine analogs with a xanthene core extended by one or two benzene rings provide this feature. Moreover, they have absorption and emission spectra shifted to the red in comparison with the "parent" rhodamines. ${ }^{[72]}$<smiles>[R2]c1ccc(C(=O)[O-])c(-c2c3ccc4cc(N)ccc4c3oc3c2ccc2cc(=N)ccc23)c1</smiles>

238 239-R<smiles>N=c1ccc2c(-c3ccccc3C(=O)[O-])c3ccc(N)cc3oc-2c1</smiles>

Rhodamine 110

Figure 27 Seminaphthorhodamine 238, naphthorhodamines 239-R and parent dye Rhodamine 110.

Seminaphthorhodamine 238 (Figure 27) displays an absorption with a maximum at $535 \mathrm{~nm}$ (in a mixture of DMSO with aq. phosphate buffer, 1:9) which corresponds to a 
bathochromic shift of $40 \mathrm{~nm}$ relative to parent Rhodamine 110. Its emission with a maximum at $628 \mathrm{~nm}$ (100 nm to the red from Rhodamine 110, Stokes shift $93 \mathrm{~nm}$ ) exhibits a moderate QY of $19 \%$. In the case of naphthorhodamine $239-\mathrm{H}$, the introduction of an additional benzene ring results in further shifts of absorption and emission maxima to 578 and $668 \mathrm{~nm}$, respectively (Stokes shift $98 \mathrm{~nm}$, QY 10\%), which are comparable to commercially available naphthofluorescein 217-COOH (Figure 24) which absorbs and emits at 595 and $660 \mathrm{~nm}$, respectively.

In order to take advantage of large Stokes shifts displayed by coumarins and strong fluorescence emission in the orange or red regions exhibited by rhodamines, hybrid compounds combining structural features of both fluorophores were designed. ${ }^{[73]}$

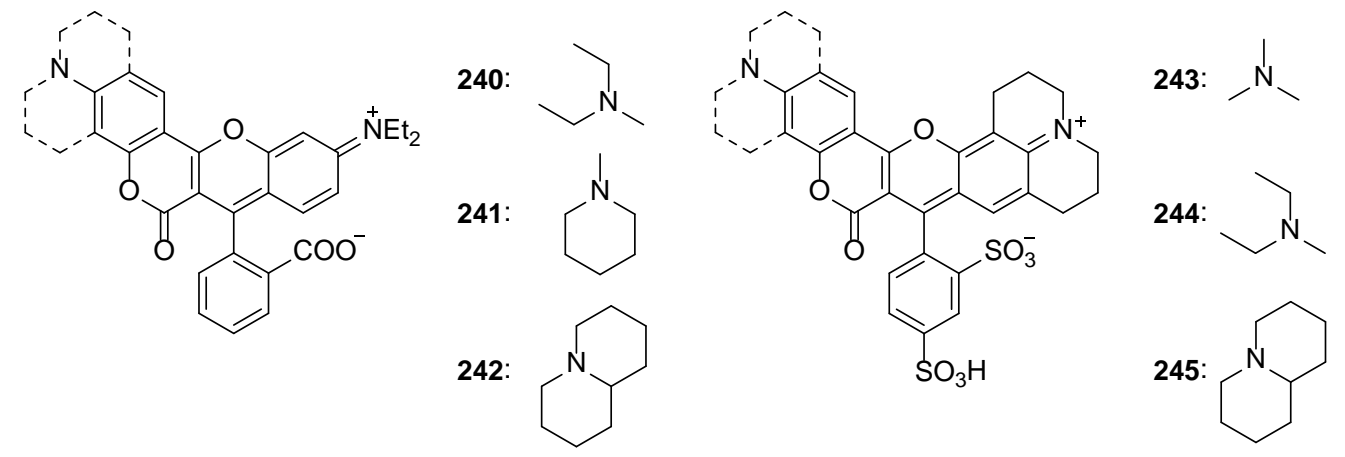

Figure 28 Rhodamine-coumarin hybrids 240-245.

The emission and absorption spectra of compounds 240-242 (Figure 28) are sensitive to the acidity of the environment (due to the formation of the colorless and non-fluorescent spirocyclic forms under neutral and basic conditions). Moreover, at $\mathrm{pH}>8$ the coumarin ring opens. In solutions acidified with TFA (to prevent the formation of spirolactones) these compounds showed larger Stokes shifts (52-72 nm in water) than those for "normal” rhodamines. However, emission efficiencies in aqueous media were very low $(<1 \%)$, possibly, due to the formation of aggregates. Spectral properties of hybrid dyes 243-245 are comparable to those of compounds 240-242. However, due to the presence of two highly polar sulfonic acid groups in the meso-phenyl ring, which prevent the formation of aggregates, fluorescence QYs are considerably higher (9-10\%). 


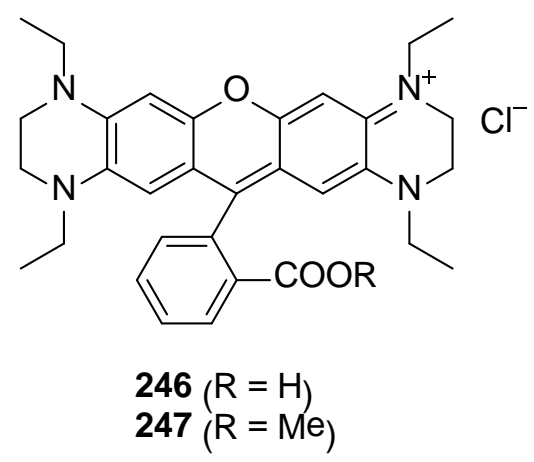

Figure 29 Rhodamine dyes 246 and 247 with 1,2,3,4-tetrahydropyrazine moieties.

Surprisingly, an introduction of two 1,2,3,4-tetrahydropyrazine moieties to the xanthene scaffold shifts absorption and emission bands towards longer wavelengths and also greatly increases the Stokes shift (Figure 29). ${ }^{[74]}$ The authors attributed this effect to an excited-state ICT which is greatly enhanced by the strong electron donor. Thus, compounds $246\left(\lambda_{\text {abs,max }} / \lambda_{\text {flu,max }}=588 / 656 \mathrm{~nm}\right)$ and $247\left(\lambda_{\text {abs,max }} / \lambda_{\text {flu,max }}=597 / 662 \mathrm{~nm}\right)$ have the largest Stokes shifts ever reported for rhodamine dyes (68 and $65 \mathrm{~nm}$ in 10\% aqueous EtOH, respectively). For comparison, Rhodamine 6G exhibits a two-times smaller Stokes shift of $30 \mathrm{~nm}\left(\lambda_{\mathrm{abs}, \max } / \lambda_{\mathrm{flu} \text {,max }}=525 / 555 \mathrm{~nm}\right)$ under the same conditions. Similarly to dyes 240-242, compound 246 exists in an equilibrium with its spirolactone form which is colorless. The position of this equilibrium is very sensitive to solvent polarity. In contrast to 246, compound 247 in which the carboxylic acid function is blocked, cannot form the corresponding spirolactone. Unfortunately, fluorescence QYs in polar solvents were not reported for these compounds.

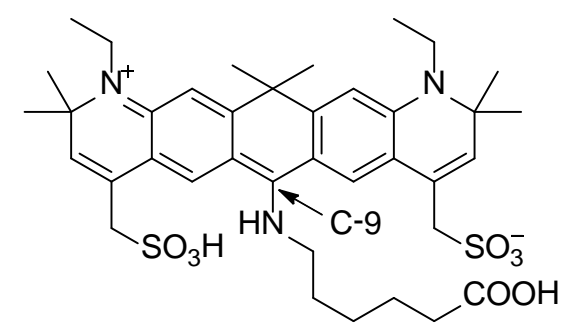

248<smiles>O=C(O)CCCCCNC1=c2cc3c4c(c2Oc2c1cc1c5c2CCCN5CCC1)CCC[N+]=4CCC3</smiles>

249

Figure 30 Meso-aminosubstituted carbopyronine 248 and pyronine 249.

Substitution of the meso-position in carbopyronine or pyronine fluorophores with a primary amine results in a fundamental change of their photophysical properties. ${ }^{[75]}$ For 
example, compounds 248 and 249 (Figure 30) in aqueous phosphate buffer absorb at 490 and $454 \mathrm{~nm}$, respectively. ${ }^{[76]}$ This corresponds to an approximately $100 \mathrm{~nm}$ hypsochromic shift compared to the parent carbopyronine and pyronine unsubstituted at C-9. Furthermore, the introduction of amine substituents provides very large Stokes shifts $(180 \mathrm{~nm}$ and $114 \mathrm{~nm}$ for compounds 248 and 249) retaining high fluorescence QYs in aq. media (26\% for $\mathbf{2 4 8}$ and $60 \%$ for $\mathbf{2 4 9}$ ). Such combination of large Stokes shifts with high emission efficiencies in polar protic media is especially rare which makes these dyes truly unique. Interestingly, the introduction of secondary amino or primary arylamino substituents leads to substantially lower QYs of fluorescence.

Boron-dipyrromethene (BODIPY) fluorophores have a strong absorption of visible light, high fluorescence QYs and good photostability. Despite these attractive properties, very small Stokes shifts commonly displayed by many BODIPY dyes (ca. $10 \mathrm{~nm}$ ) are disadvantageous to many applications including fluorescence microsopy. The reason is a so-called inner-filter effect, that is to say, the reduction of the emission intensity by selfabsorption. On the other hand, BODIPYs have a great potential for synthetic modification. ${ }^{[77]}$ Therefore, many properties, such as the positions of absorption and emission maxima, the Stokes shift or hydrophilicity, can be readily tuned to a certain extent. However, increasing the Stokes shift for BODIPY dyes is not trivial. A typical synthetic modification of the BODIPY core, such as extending the $\pi$-conjugation chain, leads to bathocromic and bathofluoric shifts, but does not increase the Stokes shift. Chen et al. ${ }^{[78]}$ overcame this issue using a new approach. They hypothesized that by introducing thienyl substituents into positions 2 and 6 of the BODIPY core (see Figure 31 for the numbering scheme of the BODIPY fluorophore), a greater extent of the geometry relaxation upon photoexcitation could be achieved. A substantial amount of the excitation energy would dissipate through rotating thienyl groups during this process, and this would effectively lower the energy of the excited state. As a result, the emission energy would become much smaller than the excitation energy, thus increasing the Stokes shift.

Compound 250 with one thienyl moiety at C-2 (Figure 31) displays the absorption and emission maxima at 507 and $603 \mathrm{~nm}$ (in 75\% aq. $\mathrm{MeOH}$ ), respectively. This corresponds to an enormous (for BODIPY dyes) Stokes shift of $96 \mathrm{~nm}$. The absorption and emission maxima of the symmetrical BODIPY derivative 251 were observed at 523 and $612 \mathrm{~nm}$, respectively (the Stokes shift is $89 \mathrm{~nm}$ ). Asymmetrical compound $\mathbf{2 5 0}$ exhibited a much stronger absorption ( $\varepsilon=65000 \mathrm{M}^{-1} \mathrm{~cm}^{-1}$ vs only $17000 \mathrm{M}^{-1} \mathrm{~cm}^{-1}$ for 251 ). However, the 
emission efficiencies displayed by both compounds in protic solvents were rather low (< $10 \%)$. Interestingly, the introduction of phenyl substituents instead of thienyls into the same positions does not increase the Stokes shift. Similarly, the substitution with thienyl moieties at C-3, C-3,5 or C-1 also conserves small Stokes shifts observed for BODIPY dyes.

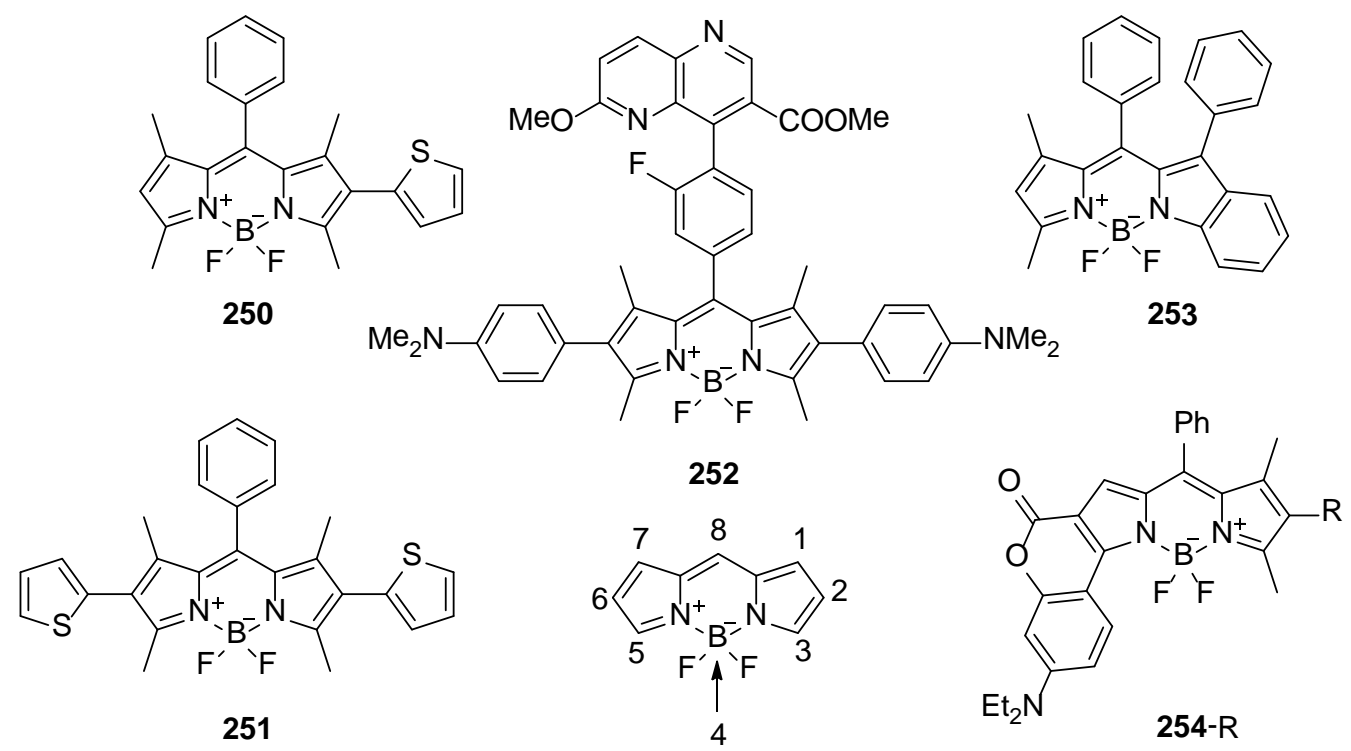

Figure 31 Large Stokes shift BODIPY dyes 250-254 and the atom numbering of the BODIPY fluorophore.

A Stokes shift of $186 \mathrm{~nm}\left(\lambda_{\mathrm{abs}}=554 \mathrm{~nm}, \lambda_{\mathrm{em}}=740 \mathrm{~nm}\right.$ in $\left.\mathrm{CH}_{2} \mathrm{Cl}_{2}\right)$ was observed for compound 252, and it represents the largest Stokes shift ever reported for BODIPY dyes. ${ }^{[79]}$ DFT calculations combined with resonance Raman spectroscopy showed that an ICT from the $p-N, N$-dimethylaminophenyl ring to the naphthyridyl unit is responsible for the lowest energy optical transition. Unfortunately, the fluorescence intensity in $\mathrm{CH}_{2} \mathrm{Cl}_{2}$ is very low $\left(\Phi_{\mathrm{fl}}=6 \%\right)$ and almost negligible in polar solvents $\left(\mathrm{MeOH}, \mathrm{MeCN}, \mathrm{H}_{2} \mathrm{O}\right)$. Asymmetric benzo[b]fused BODIPY 253 was reported to show a broad absorption band centered at $512 \mathrm{~nm}$ and an emission band with a maximum at $655 \mathrm{~nm}$ (in $\mathrm{CH}_{2} \mathrm{Cl}_{2}$ ) resulting in a large Stokes shift of $143 \mathrm{~nm} .^{\text {[80] }}$

Annelation of the coumarin unit with the BODIPY core was expected to shift absorption and emission maxima to the red spectral region and enlarge the Stokes shift compared with typical non-annelated BODIPY dyes. ${ }^{[81]}$ Indeed, $N, N$-diethylamino-substituted dyes 254-H and 254-Et, in $\mathrm{MeOH}$, exhibit the absorption maxima at around $590 \mathrm{~nm}$ and emit in the NIR region (720 and $724 \mathrm{~nm}$, respectively, Stokes shift of $130 \mathrm{~nm}$ ). In polar sol- 
vents, such as DMF or $\mathrm{MeOH}$, fluorescence is significantly quenched ( $\Phi_{\mathrm{fl}}=3-6 \%$ ). The authors attributed this behavior to the formation of a twisted ICT excited state which they considered to be non-emissive in the case of 7- $N, N$-dialkylaminocoumarins.

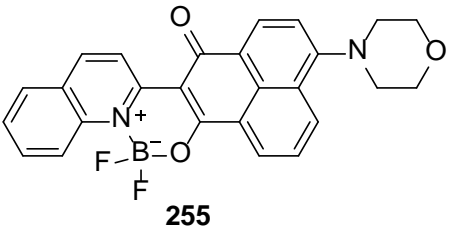

255

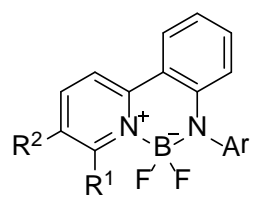

$258\left(R^{1}=R^{2}=H ; A r=P h\right)$

$259\left(R^{1}=R^{2}=H ; A r=\operatorname{dipp}\right)$

$260\left(R^{1}=M e, R^{2}=H, A r=d i p p\right)$

$261\left(\mathrm{R}^{1}=\mathrm{R}^{2}=-\left(\mathrm{CH}_{2}\right) 4^{-} ; \mathrm{Ar}=\operatorname{dipp}\right)$
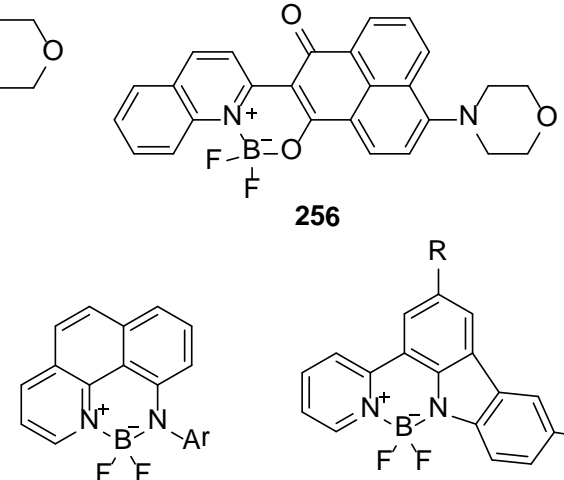

262-Ar $(A r=P h$ or dipp)

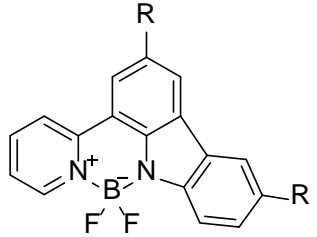

263-R $(\mathrm{R}=\mathrm{H}$ or $t \mathrm{Bu})$

dipp = 2,6-diisopropylphenyl

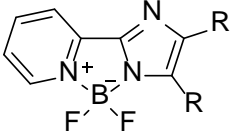

257-R $(\mathrm{R}=\mathrm{H}, \mathrm{Br}$ or $\mathrm{Ph})$

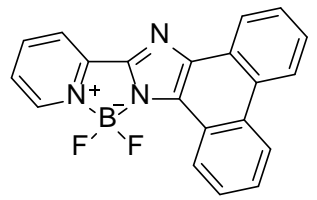

264

Figure 32 Fluorescent boron-fluorine complexes 255-264 with large Stokes shifts.

Isomeric compounds 255 and 256 (Figure 32) represent donor-acceptor complexes in which quinoline and phenalene-1,3-dione coordinate a $\mathrm{BF}_{2}$ fragment and form a strong electron-acceptor moiety, while the morpholine part acts as a donor. ${ }^{[82]}$ Similar to all fluorophores displaying ICT upon excitation, spectral properties of these compounds significantly vary with solvent polarity. Interestingly, such a minor structural change as the change in the position of the donor morpholine fragment leads to quite different photophysical properties. Thus, in $\mathrm{MeOH}$, absorption and emission maxima for compound 255 were observed at 419 and $564 \mathrm{~nm}$, respectively, and the separation between them corresponds to a large Stokes shift of $145 \mathrm{~nm}$. For the complex 256, in the same solvent, the absorption maximum was observed at $461 \mathrm{~nm}$, while the emission band had a maximum at $558 \mathrm{~nm}$, i.e. the Stokes shift was significantly smaller (97 nm) compared with isomer 255. The red-shifted emission band, the larger Stokes shift and broader emission band are consistent with a more efficient ICT process in the case of isomer 255. Both compounds 255 and 256 exhibited high fluorescence QYs in polar protic $\mathrm{MeOH}$, which is quite unusual for substances with such large Stokes shifts.

Asymmetrical anilido-pyridine boron difluoride dyes 258-261, 262-Ar and 263-R (Figure 32) were specially designed to improve very small Stokes shifts of BODIPY dyes. ${ }^{[83]}$ 
Compounds 258-261 absorb in the violet region of the visible specrum $\left(\lambda_{\mathrm{abs}, \max }=\right.$ 416-419 $\mathrm{nm}$ in $\mathrm{CH}_{2} \mathrm{Cl}_{2}$ ). They have large Stokes shifts in the range of 95-114 nm and moderate QYs (27-33\%). More rigid structures of 262-Ar and 263-R provided an efficient emission (QYs 60-83\%) retaining large Stokes shifts. These dyes also showed exceptional photostability. Thus, under conditions, when Rhodamine 101 bleached in less than $2 \mathrm{~h}$, the absorption profile of 258 remained essentially unchanged after $10 \mathrm{~h}$ of irradiation, dyes 262-Ph and 262-dipp were photostable for $16 \mathrm{~h}$, and dyes with the carbazole fragment $(263-\mathrm{H}$ and $263-\mathrm{Bu})$ retained ca. $80 \%$ of their absorbance intensity after this time period.

Boron 2-(2-pyridyl)imidazole (BOPIM) complexes also exhibited larger Stokes shifts in contrast to BODIPYs. Compound 257-H absorbs UV-light with a maximum absorbance at $293 \mathrm{~nm}$ and possesses a large Stokes shift of $66 \mathrm{~nm}\left(\lambda_{\mathrm{em}, \max }=362 \mathrm{~nm}\right)$ and a small fluorescence QY of 3\% in $\mathrm{MeOH}^{[84]}$ Interestingly, the introduction of two bromine atoms (compound 257-Br) leads to a bathocromic shift of $64 \mathrm{~nm}\left(\lambda_{\mathrm{abs}, \max }=357 \mathrm{~nm}\right.$ ), a larger Stokes shift of $130 \mathrm{~nm}\left(\lambda_{\mathrm{em}, \max }=487 \mathrm{~nm}\right)$ and a 10-fold increase in the fluorescence QY $\left(\Phi_{\mathrm{fl}}=31 \%\right)$. Absorption maxima of compounds 257-Ph and 264 are further red-shifted (391 and $404 \mathrm{~nm}$, respectively). ${ }^{[85]}$ Their emission maxima are at 525 and $531 \mathrm{~nm}$, which correspond to Stokes shifts of 134 and $127 \mathrm{~nm}$, respectively. Both compounds show moderate emission efficiencies in $\mathrm{MeOH}$ (12\% and 14\%). All BOPIM complexes displayed negative solvatochromism. More polar solvents shift the lowest-energy absorbtion bands towards the blue region of the UV-vis spectrum. This indicates that the $S_{0} \rightarrow S_{1}$ absorption band has an ICT character, and the energy of the ground state decreases more than that of the excited state with increasing solvent polarity, and this effect makes the energy gap larger.

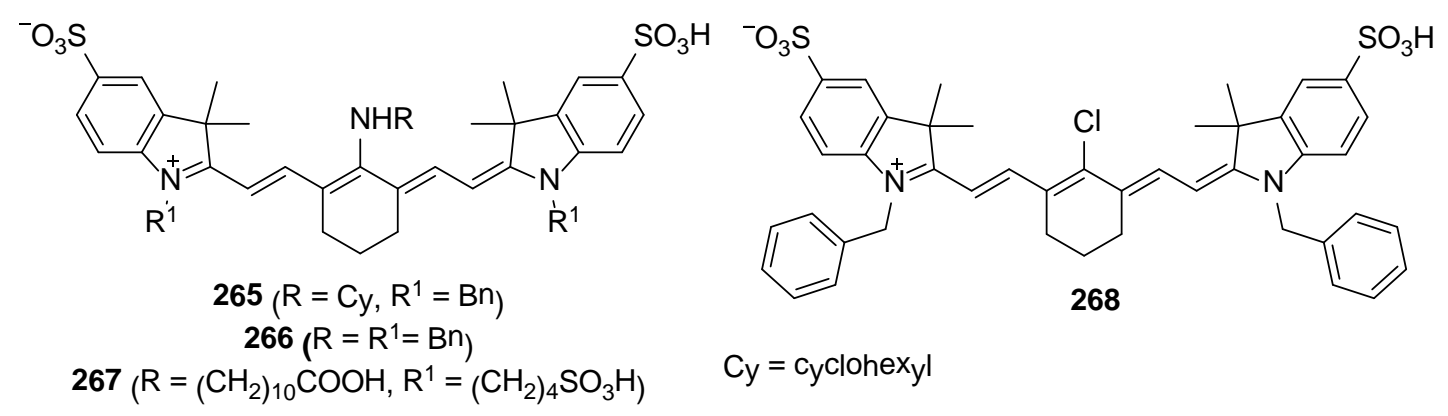

Figure 33 Heptamethine dyes 265-267 with large Stokes shift and their precursor 268. 
Heptamethine dyes 265-267 have an amino substituent attached to the methine chain (Figure 33). In this respect they are similar to compounds 248 and 249 with a C-N bond in the meso-position of carbopyronine and pyronine fluorophores. Heptamethine dyes 265-267 show very interesting spectral properties. Compared with precursor cyanine dyes, these compounds have larger Stokes shifts and high fluorescence QYs in water. For example, compounds 265-267 have broader spectra and much stronger fluorescence than dye 268. Their solutions in water are blue-colored $\left(\lambda_{\mathrm{abs}, \max }=600-617 \mathrm{~nm}\right)$, whereas solutions of compound 268 are green, which corresponds to a hypsochromic shift of 180-190 nm. ${ }^{\text {[86] }}$ Fluorescent dyes $\mathbf{2 6 5}-\mathbf{2 6 7}$ emit in the NIR region with large Stokes shifts of 140-155 nm (in water). However, emission efficiencies of 265 and 266 are rather low ( $7 \%)$. Luckily, the introduction of two additional sulfonate residues results in a good fluorescence QY (37\% for compound 267) in aqueous solutions. In addition, compound 267 (also known as 4-Sulfonir) has a large molar absorptivity of $1.8 \times 10^{5} \mathrm{M}^{-1} \mathrm{~cm}^{-1}$, which together with the large QY provides high brightness in microscopy applications.

\subsubsection{Fluorophores with a Stokes shift provided by photochemical processes}

All compounds described in the previous section exhibited large Stokes shifts as a result of photophysical processes such as intramolecular charge transfer (ICT), solvent relaxation around a large excited dipole and steric relaxation of compounds with bulky groups. In contrast to these processes, there are other mechanisms providing large Stokes shifts. They involve adiabatic photochemical reactions leading to an emissive product with a chemical structure different from that of the starting molecule. A short overview of various photochemical mechanisms, such as excimer/exciplex formation, excited state intramolecular proton transfer (ESIPT) and twisted ICT (TICT), ${ }^{[87]}$ providing large Stokes shifts is given below.

A molecule in the excited state can form a complex with an identical molecule (in the case of excimer formation) or a different molecule (in the case of exciplex formation) in the ground state. The formation of these complexes may quench the emission, ${ }^{[88]}$ but sometimes an excimer/exciplex can be emissive. For example, many aromatic hydrocarbons such as naphthalene or pyrene form excimers. The fluorescence band which corresponds to an excimer is red-shifted relative to the monomer and does not show vibronic 
structure. The so called intramolecular excimers have two identical fluorophores which are linked by a short flexible chain. The formation of excimers or exciplexes involves diffusion processes or large intramolecular rearrangements. Therefore, it is diffusioncontrolled and can be used to probe the local fluidity of the medium. ${ }^{\left[{ }^{[9]}\right.}$ For example, a concentration-dependent ability of the BODIPY label to form excimers with red-shifted emission was used to study the lipid transport and metabolism. ${ }^{[90]}$ The dual probes consisting of pyrene fluorophores attached to oligonucleotides were used for the detection of a single-base point mutation. ${ }^{[91]}$ In the course of adjacent binding of the modified nucleotides to a complementary target sequence, a pronounced spectral change from the blue monomer emission to the green excimer emission with a larger Stokes shift occurred ( 140 nm) allowing a simple detection with a spectrofluorimeter. A similar approach used an exciplex formation between 1-pyrenemethylamine and $N$-methyl- $N$-naphthalene1-ylethanediamine fragments attached to the 5'- or 3'-terminal phosphate groups of oligonucleotides upon binding to target sequence of a nucleic acid. ${ }^{[92]}$ The huge Stokes shift (100-150 nm) exhibited by this probe allowed detection of a single nucleotide polymorphism (SNP) DNA by a naked eye. Unlike other fluorescence probe approaches (e.g., FRET), the formation of excimers and exciplexes is more sensitive to the correct assembling of counterparts.

Incorporation of multiple fluorophores into the DNA scaffold can result in a multifluorrophoric system with interesting photophysical properties (see review ${ }^{[93]}$ ). For example, various fluorophores were introduced at C-1' of the D-ribose to replace nucleobases and produce artificial fluorescent deoxynucleosides (deoxyfluorosides). ${ }^{[94]}$ Afterwards, combinatorial libraries were prepared to reveal oligodeoxyfluorosides (ODFs) with best photophysical properties in the single-stranded state. In some ODFs, fluorophores formed excimers or exciplexes which emitted fluorescence with large Stokes shifts (up to 140 $\mathrm{nm}$ ) and possessed large QYs in aq. buffers. In another case, a pyrene dye was attached to the C-2' position of D-ribose in the nucleotide of interest. ${ }^{[95]}$ In a molecule of the duplex RNA containing the modified nucleotide, the pyrene moiety resides outside the doublehelical structure in the minor groove. When multiple pyrenes were introduced consecutively into the duplex RNA, strong excimer emission at around $480 \mathrm{~nm}$ (cf. $396 \mathrm{~nm}$ for the monomer) with a high QY was observed. Interestingly, with an increase in the number of incorporated pyrenes, the excimer fluorescence increased faster than linearly. 
In the case of the excited state intramolecular proton transfer (ESIPT), a molecule in the excited state undergoes tautomerization, i.e. proton transfer from one basic site to another in the same molecule. The ESIPT reaction requires the formation of a hydrogen bond between a proton donor (hydroxyl, imino or amino group) and an acceptor group (for example, carbonyl or pyridine) which are located in close proximity. Upon excitation, a proton transfer takes place (acid-base properties of the excited state are different), forming a tautomer in the excited state $\mathrm{S}_{1}$, the geometry and electron distribution of which significantly differs from its corresponding normal species in the excited state $\mathrm{S}_{1}$ (Scheme 2). ${ }^{[96]}$ The structural change during the ESIPT process may lead to unusually large Stokes shifts. Unfortunately, in protic solvents, the ESIPT emission is usually inhibited, and therefore, only fluorescence from the "normal" tautomer with a small Stokes shift is observed. Moreover, extending of the $\pi$-conjugation - a usual strategy for design of new fluorophores - may inhibit the ESIPT process. ${ }^{[97]}$

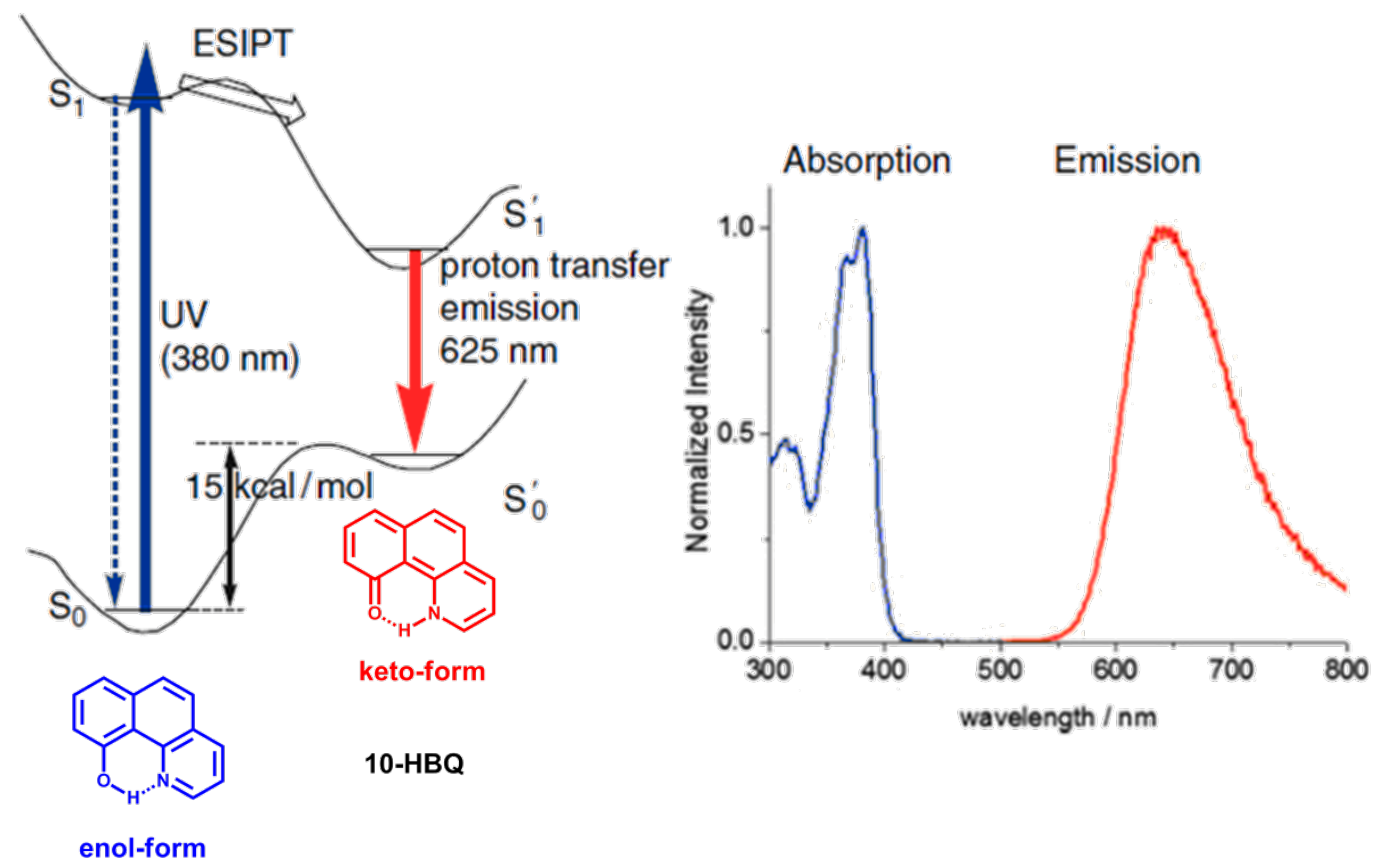

Scheme 2 ESIPT process in 10-hydroxybenzo[h]quinoline. ${ }^{[96]}$

Another process which could produce a large Stokes shift is the formation of twisted ICT states (TICT), which involves mutual twisting (in the excited state) of donor and acceptor parts of a molecule connected by a single bond and simultaneous charge transfer between them. In the TICT system, a donor D and an acceptor A are coplanar in the ground state, and the same coplanar arrangement is retained in the initially reached Franck-Condon 
excited state. Due to the large $\pi$-overlap, mesomeric interaction between D and A is significant, and excitation can involve a (partial) charge transfer. In addition to an $\mathrm{S}_{1}$ minimum for the planar geometry these systems can possess another low lying $\mathrm{S}_{1}$ minimum for the perpendicularly twisted geometry with full charge separation, the TICT state. The latter can be populated in an adiabatic photoreaction which combines an excited-state intramolecular twist, electron transfer and solvent reorganization. ${ }^{[98]}$ For example, $4-N, N$ dimethylaminobenzonitrile (DMABN) was found to exhibit a dual fluorescence in polar solvents. The emission spectrum of this compound consists of a "normal" band $\left(\lambda_{\mathrm{abs}, \max }=\right.$ $361 \mathrm{~nm}$, in $\mathrm{MeCN}^{[99]}$, which is analogous to emission bands exhibited by closely related benzene derivatives, and an “anomalous” band $\left(\lambda_{\mathrm{abs}, \max }=492 \mathrm{~nm}\right.$, in $\left.\mathrm{MeCN}^{[99]}\right)$ with a large Stokes shift. These bands were ascribed to the excited state with the planar geometry (locally excited) and the TICT state with the twisted dimethylamino group, respectively. ${ }^{[100]}$ When the twisting of the dimethylamino group is hindered, as in the case of compound 270, formation of the TICT state is impossible, and only the "normal" emission band is observed. At the same time, compound 271, where the amino group is fixed in the twisted conformation, exhibits only fluorescence with a large Stokes shift (from the TICT state). ${ }^{[101]}$

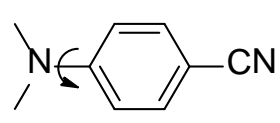

269

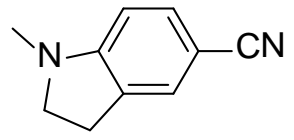

270

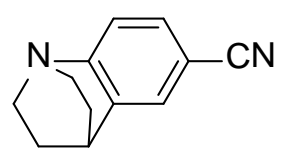

271

Figure $34 p$ - $N, N$-Dimethylaminobenzonitrile 269 which undergoes TICT in the excited state and model compounds 270 and 271.

As a rule, emission efficiencies of compounds displaying the TICT are very low. In fact, many TICT states were found to be non-emissive and responsible for the rapid non-radiative decay of numerous important dyes (for example, in the case of 7$N, N$-dialkylaminocoumarins ${ }^{[21 \mathrm{~d}, 21 \mathrm{e}, 21 \mathrm{~g}, 35]}$ ) which leads to intramolecular fluorescence quenching. The latter effect may be due to the reduced value of the transition dipole moment (for transition $S_{1} \rightarrow S_{0}$ ) which results from the lack of the orbital overlap between $\mathrm{D}^{+}$and $\mathrm{A}^{-}$moieties. ${ }^{[102]}$ Therefore, a rational design to attain the TICT emission with a large Stokes shift and a high QY at the same time still remains a challenge. 


\subsubsection{Multifluorophore constructs with pseudo-large Stokes shifts}

In order to address the problem of small Stokes shifts exhibited by most of the conventional fluorescent dyes, resonance energy transfer (RET) between two organic fluorophores, donor and acceptor, connected by a suitable linker, can be utilized to obtain dyades with pseudo-large Stokes shifts. Photophysical properties and applications of such molecular constructs, called energy transfer cassettes, have been reviewed recently. ${ }^{[103]}$ When both fluorophores are connected by a non-conjugating linker, the energy transfer occurs through space (Scheme 3). This process is usually called fluorescence resonance energy transfer (FRET) and occurs through non-radiative dipole-dipole coupling. According to the FRET theory, ${ }^{[104]}$ several requirements should be fulfilled for efficient FRET: (1) high fluorescence QY of the donor, (2) a substantial overlap between the donor emission band and the acceptor absorption band, (3) an appropriate alignment of the absorption and emission transition moments and their separation vector and (4) a sufficiently short distance (typically less than $10 \mathrm{~nm}$ ) between the donor and acceptor.



Scheme 3 Energy transfer cassette based on FRET. ${ }^{[105]}$

For example, FRET pairs 272 and 273 (Scheme 4) consist of the donor coumarin and the acceptor rhodamine dyes. Being excited in aqueous phosphate buffer at wavelengths where the coumarin donor moiety absorbs, they do not show any emission of the donor (coumarin) part. Instead, strong emission of the rhodamine acceptor around $590 \mathrm{~nm}$ was observed. ${ }^{[106]}$ This behavior is consistent with the very efficient intramolecular energy transfer between two dyes (with energy transfer efficiencies $E$ 96.1\% and 97.3\%, respectively). The rigid piperazinyl linker connecting two fluorophores was found to be important for the efficient energy transfer. Thus, pseudo-large Stokes shifts of around 170 nm with good fluorescence QYs in aqueous media were obtained, a result which otherwise would be extremely difficult to realize using only one fluorophore. The drawback of 
this approach is that an emission can be caused by exciting not only the donor, but also the acceptor part of the dyad.

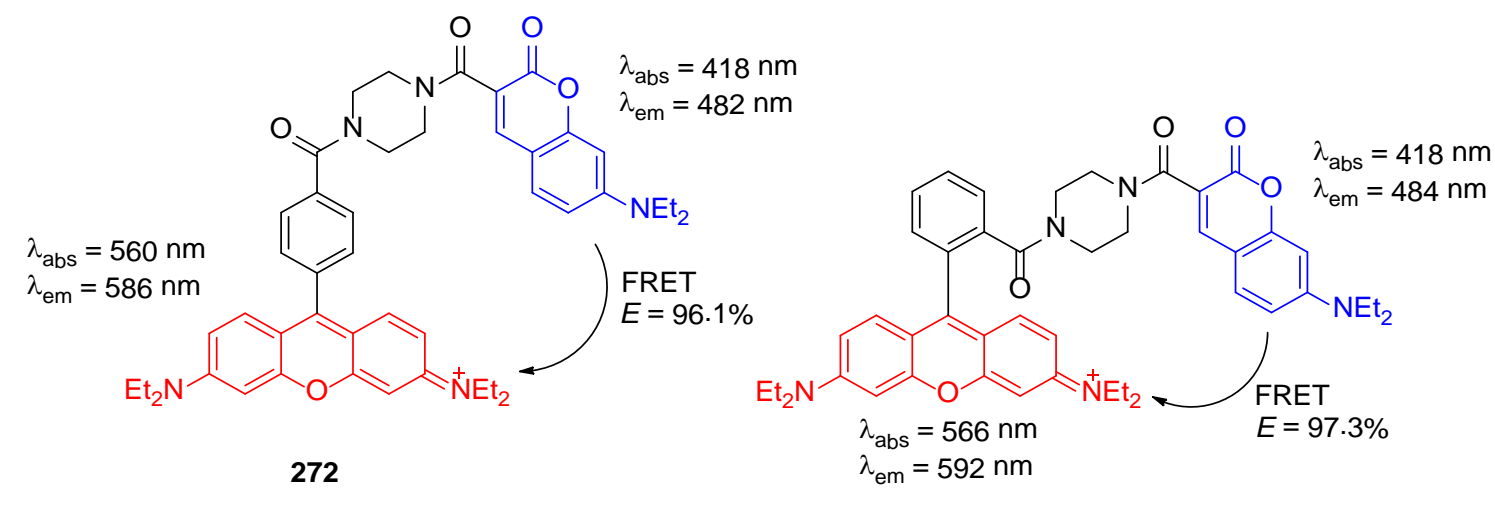

273

Scheme 4 FRET in 7- $N, N$-Diethylaminocoumarin- $N^{\prime}, N^{\prime}, N$ ',,$N$ ''-tetraethylrhodamine dye pairs 272-273.

Although the FRET-based cassettes allow increasing apparent Stokes shift, this approach is still constrained by the requirement that the fluorescence of the donor must overlap with the absorption of the acceptor. If the donor and acceptor are linked by a $\pi$-electron system, the radiationless electronic energy transfer through bonds (Dexter and superexchange mechanisms ${ }^{[107]}$ ) and through space (Förster mechanism) may occur (Scheme 5). If the through-bond energy transfer (TBET) is fast relative to non-radiative decay pathways, then, unlike FRET-based cassettes, TBET is not constrained by this requirement. A good TBET-based cassette for labeling biological systems should meet the following requirements: (1) a strong absorbance of the donor component at the excitation wavelength, (2) a large fluorescence QY of the acceptor component, (3) the presence of a suitable linker which prevents the system from becoming planar and, therefore, from behaving as a single conjugated dye. ${ }^{[105]}$ TBET-based cassettes enable greater freedom for the selection of fluorophores than FRET-based ones. They can provide larger Stokes shifts and extend their emission further into the IR wavelength region. ${ }^{[103]}$

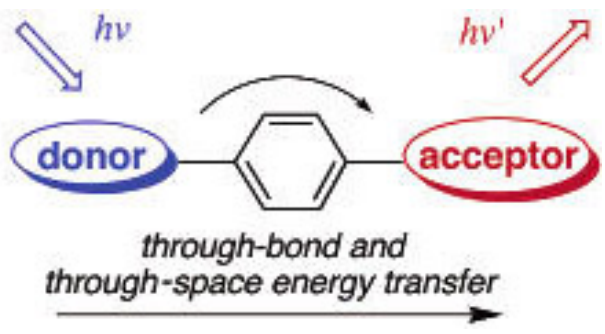

Scheme 5 Energy transfer cassette based on TBET. ${ }^{[105]}$ 
For example, compounds 274-277 (Scheme 6) display absorption maxima which are characteristic for their donor and acceptor components. They all have a fluorescein fragment and, therefore, absorb strongly around $512 \mathrm{~nm}$ (in EtOH). Upon excitation with an Ar laser at 488 or $514 \mathrm{~nm}$, these cassettes emit fluorescence at 538, 582, 603 and $616 \mathrm{~nm}$ (pseudo-Stokes shifts: 28, 70, 91 and $104 \mathrm{~nm}$ ), respectively, and almost no fluorescence from the donor, which corresponds to nearly $100 \%$ energy transfer efficiency. ${ }^{[105]}$ Furthermore, the fluorescein fragment in cassettes 274-277 was found to be considerably more stable to photobleaching than fluorescein itself which is known to be poorly photostable. In a typical experiment, cassette 276 retained 95\% of its original fluorescence level, whereas the fluorescence of fluorescein in the same solution had decreased by $55 \%$. This behavior was attributed to a fast energy transfer from the fluorescein donor fragment to the rhodamine acceptor in the cassette. As a result, intersystem crossing cannot compete with this process, and the triplet states, which are believed to be the main cause of photobleaching in case of fluorescein, are not significantly populated.

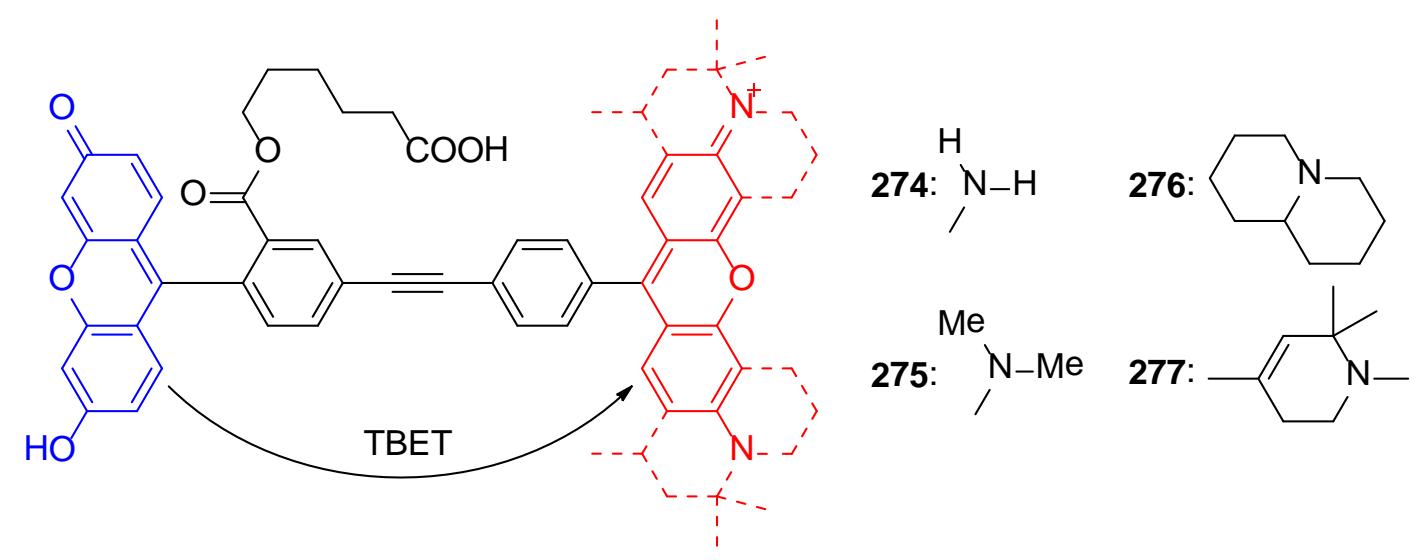

Scheme 6 TBET in fluorescein-rhodamine dye pairs 274-277.

Remarkably, cassettes 278-R and 279 (Scheme 7) have only negligible spectral overlap between the donor emission and the acceptor absorption bands. ${ }^{[108]}$ They displayed the absorption band of the rhodamine and coumarin parts at around $560 \mathrm{~nm}$ and $350 \mathrm{~nm}$ (in case of 278-R) or $370 \mathrm{~nm}$ (for 279), respectively. Upon excitation of the cassettes 278-R and 279 in a solution of phosphate buffer with $\mathrm{MeOH}$ (3:2) at the coumarin absorption maximum, only the rhodamine emission at around $582 \mathrm{~nm}$ with moderate QYs $(<27 \%)$ was observed. The absence of the coumarin emission indicates that the energy-transfer efficiencies were nearly perfect (>99\%). The pseudo-Stokes shifts of up to $230 \mathrm{~nm}$ are 
much larger than those of fluorescein-rhodamine TBET cassettes and those of the typical FRET-based rhodamine systems.
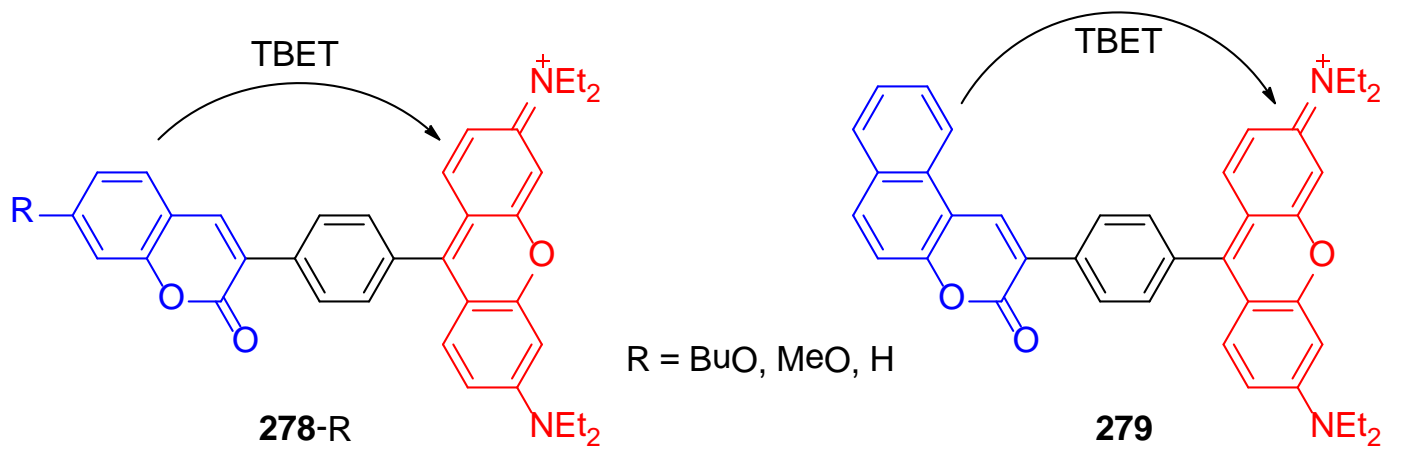

Scheme 7 TBET in coumarin- $N, N, N^{\prime}, N^{\prime}$-tetraethylrhodamine dye pairs 278-R and 279.

If the donor dye in the FRET or TBET cassette has a very high value of the fluorescence QY, this may often lead to a fluorescence "leaking” from the donor due to inefficient energy transfer. In order to overcome this problem, the "dark" resonance energy transfer (DRET) concept was proposed. ${ }^{[109]}$ In this approach, a "dark" dye with very low fluorescence QY is used as the donor component. Upon excitation, a very efficient and fast energy transfer occurs due to large spectral overlap of the donor-acceptor pair and high extinction coefficient of the acceptor, despite the low quantum yield and short excitedstate lifetime of the donor. For example, the absorption spectrum of compound $\mathbf{2 8 0}$ (Scheme 8) displayed a characteristic absorption band of the donor fragment (shown blue) at around $494 \mathrm{~nm}$ and an absorption band of the acceptor fragment at around $577 \mathrm{~nm}$ (shown red). Upon excitation of this compound with $470 \mathrm{~nm}$ light in EtOH, only the strong $\left(\Phi_{\mathrm{fl}}=0.38\right)$ and characteristic emission of the acceptor part with a maximum at $617 \mathrm{~nm}$ was observed. Transient absorption spectra showed, that the decay profile of the donor alone only exhibited a single decay component of 33 ps, whereas the decay time constant of the donor component in the DRET-pair was much shorter (1.2 ps). These data led to the conclusion that the energy absorbed by the donor is efficiently transferred to the acceptor (96\% efficiency) without quenching by any non-radiative intramolecular rotations. 


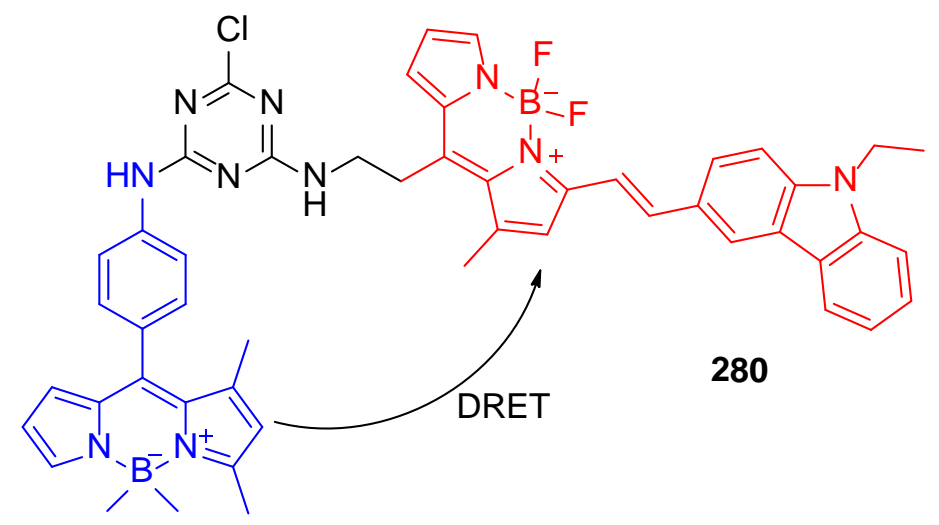

Scheme 8 DRET in BODIPY-based dye pair $\mathbf{2 8 0 .}$

\subsection{Applications of large Stokes shift dyes in fluorescence nanoscopy}

There are not so many examples of studies in which a large Stokes shift dye (or its combination with a "normal" dye) is used as a fluorescent label in optical super-resolution microscopy. This is obviously due to the lower photostability and brightness of most dyes with large Stokes shift. However, there are some modern bright and photostable large Stokes shift dyes which have been successfully applied in optical "nanoscopy". For example, the suitability of light from a multicolor stimulated-Raman-scattering (SRS) source for gaining STED resolution was successfully demonstrated with silica beads which were labeled with the commercially available coumarin dye ATTO425 and imaged in a simple custom-built STED microscope. ${ }^{[110]}$ Unlike the supercontinuum light source used in another STED microscope, ${ }^{[16]}$ the optical power of the SRS light source, which consists of a microchip laser coupled to a fiber where SRS occurred, is preserved within narrow peaks in a comb-like spectrum, the output light is linearly polarized, and light suitable for STED is produced below $630 \mathrm{~nm}$. Thus, for ATTO425, STED was performed at $532 \mathrm{~nm}$ (the fundamental wavelength from the comb spectrum of the SRS light source). Excitation of the fluorophore was accomplished using a $440 \mathrm{~nm}$ diode laser. Due to simplicity, compactness, broad spectral output and low cost, SRS sources could become an attractive option for spectrally flexible STED imaging. 
<smiles></smiles>

NK51

Figure 35 Rhodamine dye NK51.

In another report, the commercially available coumarin dye DY-485XL (see Table 1 for structure) was used together with the small Stokes shift rhodamine dye NK51 (Figure 35) to demonstrate the advantages and illustrate the principles of isoSTED (a combination of STED and $4 \mathrm{Pi}^{*}$ microscopies to improve resolution along the Z-axis). Two-color isoSTED enables imaging of the spatial distribution of two or more (biological) objects at the nanoscale in three dimensions. ${ }^{[19]}$ The coumarin dye DY-485XL displays a similar emission spectrum as rhodamine dye NK51, whereas its excitation spectrum is blueshifted by 50 nm (Figure 36). This combination of fluorophores allowed using only one STED beam at $647 \mathrm{~nm}$ and two readily available excitation lasers with emission at 488 nm (for DY-485XL) and $532 \mathrm{~nm}$ (for NK51). In this study, mitochondrial outer membrane protein Tom20 was labeled by indirect immunostaining with dye DY-485XL, and the matrix protein $\mathrm{mtHsp} 70$ - with dye NK51. The images were recorded by subsequent excitation of the sample at $488 \mathrm{~nm}$ (channel 1) and $532 \mathrm{~nm}$ (channel 2) and depletion of the fluorescence by stimulating emission at $647 \mathrm{~nm}$. The residual excitation cross-talk between two channels was efficiently removed by linear unmixing applied to the raw data. These dual-color images were the first example of the optical super-resolution microscopy providing separate images of two proteins of an organelle inside the whole cell with a nanoscale 3D resolution in the 50 -nm range for both channels.

\footnotetext{
* 4 Pi-microscope is a variation of confocal microscope, where the improvement in axial resolution achieved by using two opposing lenses focused to the same location.
} 

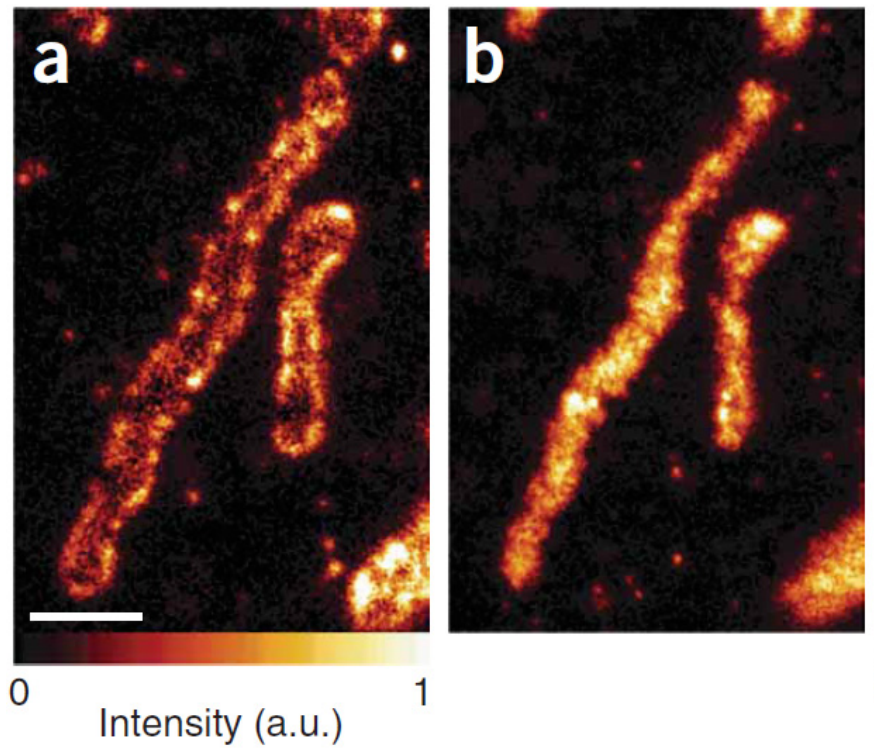

1

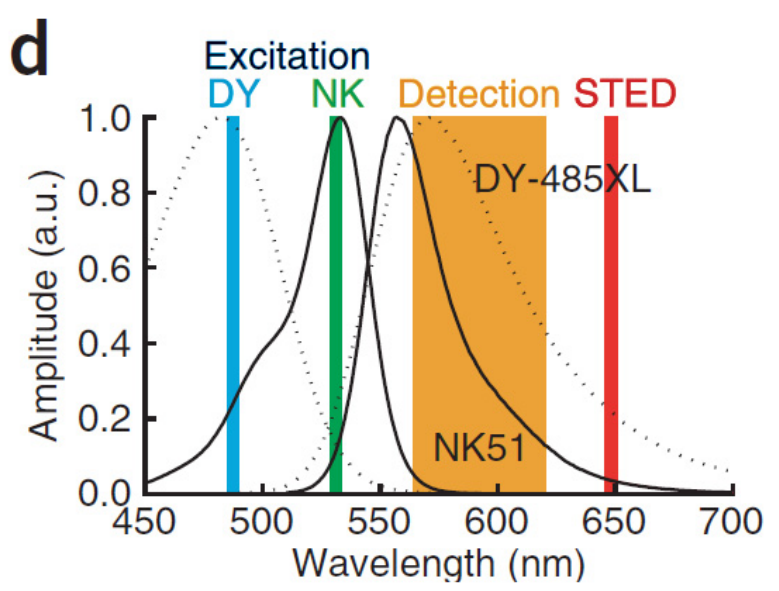

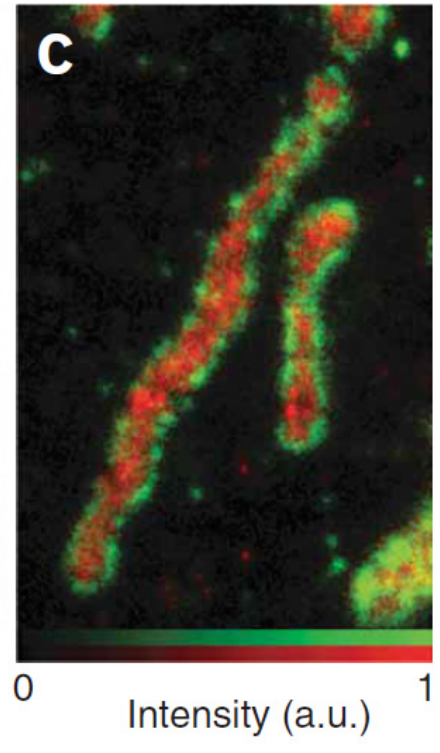

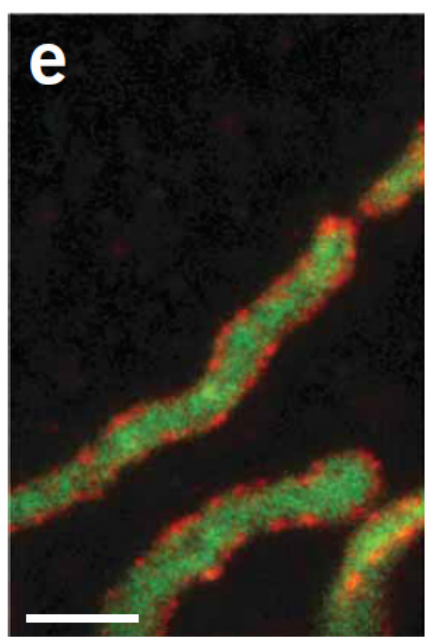

Figure 36 Two-color isoSTED imaging of mitochondria in Vero cells. ${ }^{[19]}$ (a,b) Distribution of the outer membrane protein Tom20 labeled with the organic fluorophore DY-485XL (a) and of the NK51-labeled matrix protein mtHsp70 (b). (c) Overlay of the two images showing Tom20 in green and mtHsp70 in red. (d) Excitation and absorption spectra for DY-485XL (dotted line) and NK51 (solid line). The two fluorophores are separated by subsequently exciting the sample at $488 \mathrm{~nm}$ (DY-485XL) and $532 \mathrm{~nm}$ (NK51). Both dyes are depleted by stimulated emission at $647 \mathrm{~nm}$. (e) Reverse staining as a control (Tom20 with NK51 and Hsp70 with DY-485XL) yields similar results as the initial recording (c). Scale bars $=1 \mu \mathrm{m}$.

Another commercially available coumarin dye DY-480XL with a large Stokes shift and the optical spectra similar to those of DY-485XL was used together with rhodamine dye KK114. ${ }^{[111]}$ This study dealt with an investigation of the spatial organization of the synaptotagmin 1 (Syt1) surface pool in hippocampal presynaptic boutons. For that, dualcolor isoSTED microscopy ${ }^{[112]}$ was used. In order to reveal the localization of surfacestranded Syt1 at synapses, Syt1 was stained with KK114. The synapses were identified by labeling pre- and postsynaptic markers RIM1, RIM2 (RIM) and Homer1 with the dye 
DY-480XL. The dyes were excited sequentially at fixed wavelengths of $530 \mathrm{~nm}$ (for DY480XL) and $635 \mathrm{~nm}$ (for KK114). Since both dyes have similar emission spectra, STED pulses only at one wavelength of $775 \mathrm{~nm}$ were required. Emission of both fluorophores was detected in the range of 660-700 nm (in separate channels). After deconvolution, the final super-resolved images were obtained (see Figure 37).
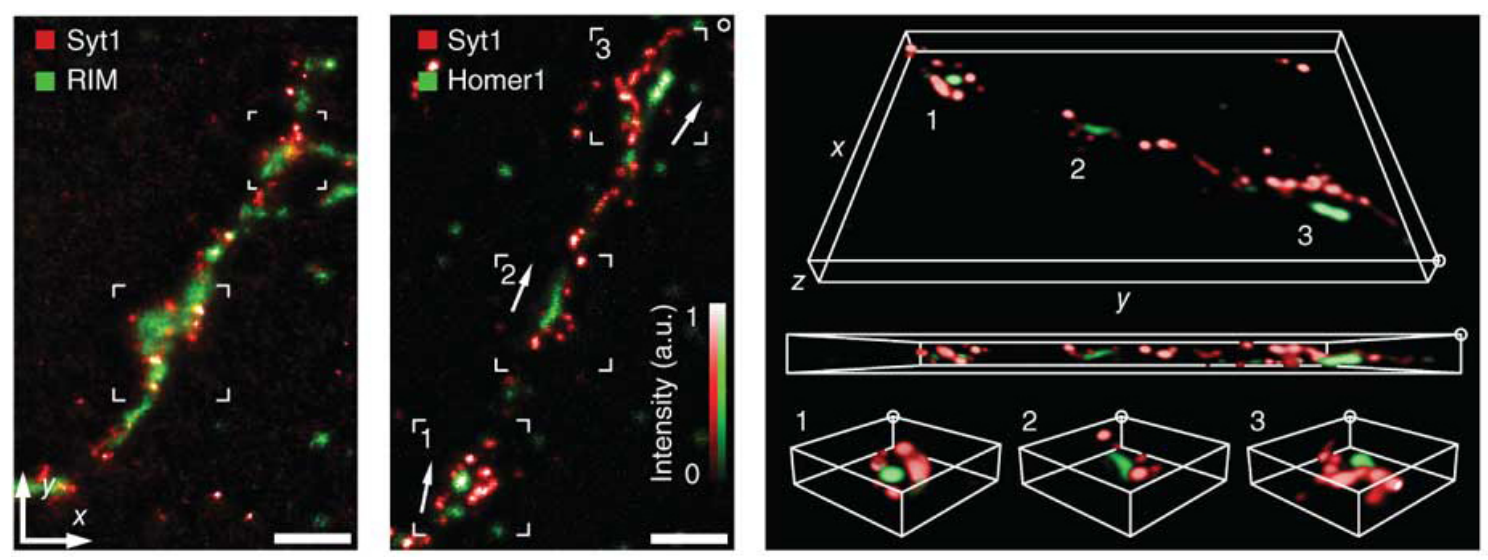

Figure 37 The localization of the surface-stranded protein Syt1 at synapses revealed by three-dimensional dual-color isoSTED nanoscopy. ${ }^{[112]}$ Syt1 was labeled with KK114 (red); postsynaptic markers RIM1, RIM2 (RIM) - with DY-480XL (green). Scale bars $=1 \mu \mathrm{m}$.

In material science, dual-color STED microscopy was successfully applied to localize and investigate the repartition of fluorescent polymer nanoparticles of ca. $100 \mathrm{~nm}$ size in nanofibers. ${ }^{[113]}$ Usually the BODIPY dyes are not used in STED microscopy due to their very small Stokes shifts (and therefore, a significant risk of re-excitation by the STED beam). However, the hydrophobic polymerizable BODIPY dye B504-MA (Figure 38) found to be applicable in STED nanoscopy. It was used to label the polymer nanoparticles, whereas the PVA matrix was stained with a water-soluble derivative of perylenetetracarboxylic acid (PTCA) which has a comparatively large Stokes shift. This combination of fluorophores allowed using the excitation by different lines of the same argon laser (458 nm for PTCA and $514 \mathrm{~nm}$ for B504-MA). The emission spectra of these fluorophores overlap considerably. Therefore, efficient depletion using a single STED beam at $592 \mathrm{~nm}$ could be achieved. The nanoparticle sizes and nanofiber thicknesses were shown to be in good agreement with the values obtained by electron microscopy. 

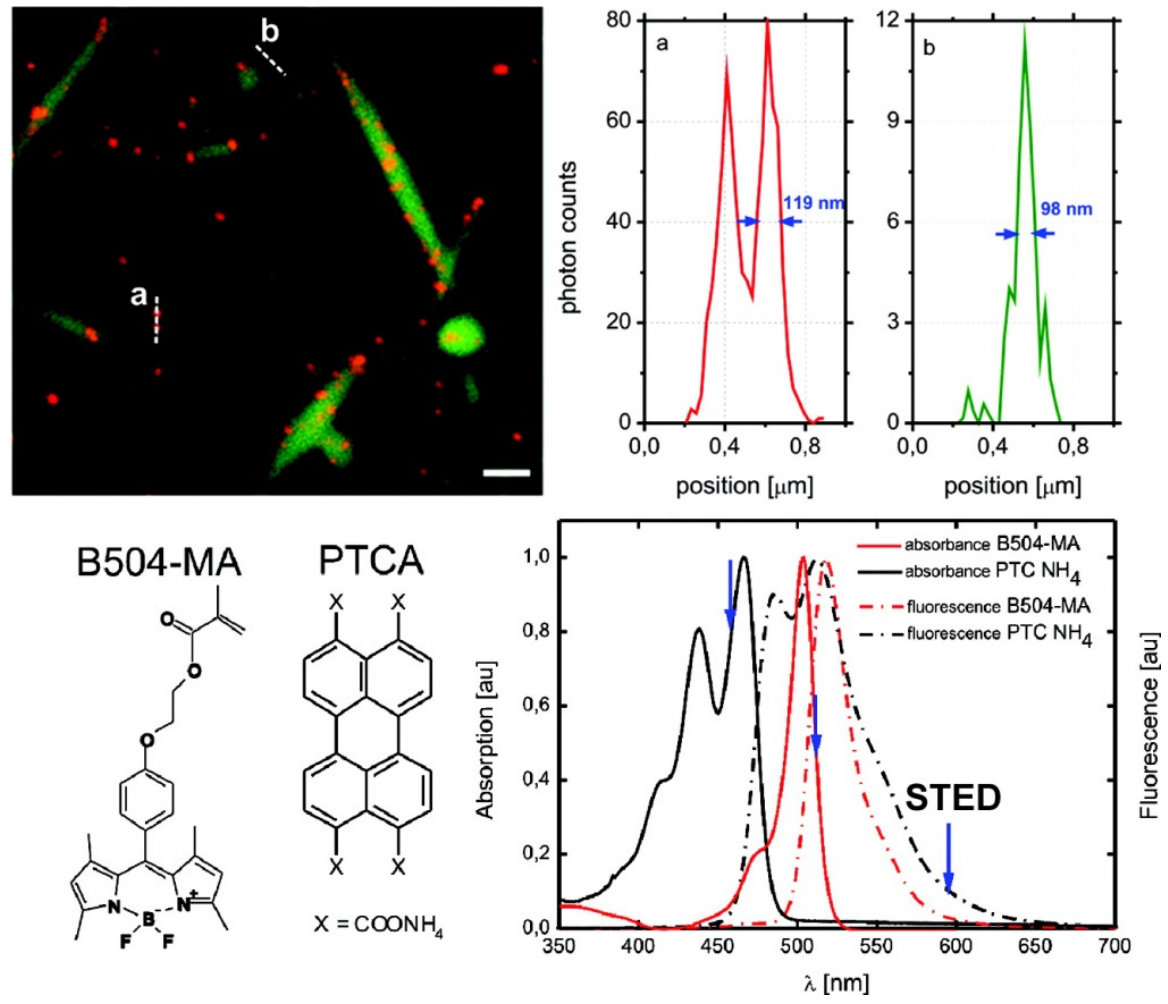

Figure 38 The STED image (raw data) of polystyrene nanoparticles (labeled with B504-MA, shown red) immobilized within PVA nanofibers (labeled with PTCA, shown green). ${ }^{[113]}$ Scale bar $=680 \mathrm{~nm}$.

One of the brightest commercially available dyes with a large Stokes shift Chromeo494* was used together with a carbopyronine dye Atto $647 \mathrm{~N}$ in a dual color STED microscopy in order to investigate the dynamics of and epidermal growth factor (EGF) epidermal growth factor receptor (EGFR) in living cells. ${ }^{[14]}$ For this purpose, a fused protein EGFR-SNAP $_{\mathrm{f}}$ expressed by HEK293 cells was labeled with BG-Chromeo494 (a conjugate of Chromeo494 with benzyl guanidine). The $\mathrm{SNAP}_{\mathrm{f}}$-sequence (polypeptide) also known as SNAP-tag specifically recognizes the BG conjugates and participates in an efficient $\mathrm{S}_{\mathrm{N}} 2$-type reaction resulting in a permanent covalent bond between $\mathrm{SNAP}_{\mathrm{f}}$ and the fluorophore. This methodology enables one to obtain the EGFR-SNAP $\mathrm{f}_{\mathrm{f}}$-Chromeo494 conjugate. On the other hand, the recombinantly expressed fused protein EGF-CLIP $\mathrm{f}_{\mathrm{f}}$ was labeled with BC-ATTO647N (conjugate of ATTO647N with benzyl cytosine). Similarly to $\mathrm{SNAP}_{\mathrm{f}}, \mathrm{CLIP}_{\mathrm{f}}$ sequence specifically recognizes BC derivatives. As a result of this reaction, the labeled exogenous protein EGF-CLIP $\mathrm{f}_{\mathrm{f}}$-ATTO647N was formed. During incubation of EGFR-SNAP f $_{\text {-Chromeo494 with EGF-CLIP }}$-ATTO647N, EGF specifically

\footnotetext{
${ }^{*}$ Nowadays, a brighter dye with similar optical spectra - Abberior Star 520SXP - is available.
} 
binds to EGFR. This resulted in the double staining of the EGFR-EGF complex with two dyes suitable for two-color STED imaging.

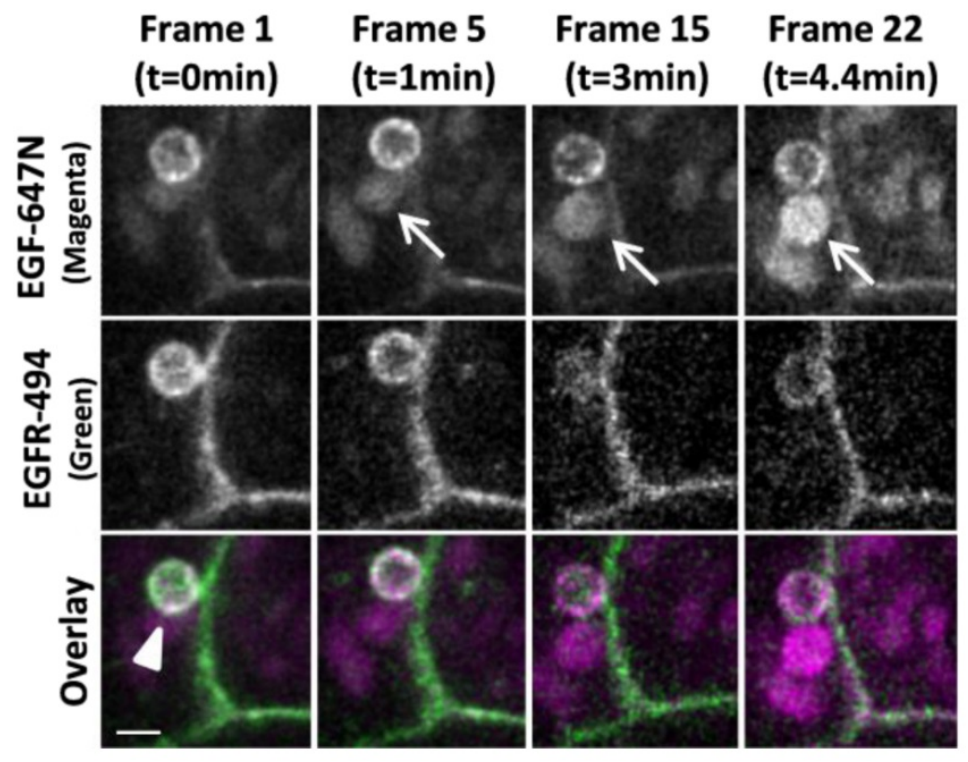

Figure 39 A time series of 2-color STED images (raw data) of living cells (HEK293) labeled with EGFCLIP $_{\mathrm{f}}$-ATTO647N (magenta) and EGFR-SNAP ${ }_{\mathrm{f}}$-Chromeo494 (green). ${ }^{[114]}$ Scale bar $=1 \mu \mathrm{m}$.

Imaging was performed by sequential excitation of fluorophores with synchronized pulsed lasers at $532 \mathrm{~nm}$ (for Chromeo494) and $640 \mathrm{~nm}$ (for ATTO647N) and depletion with a tunable mode-locked Ti:Sapphire laser at $760 \mathrm{~nm}$. A time series of STED images (see Figure 39) showed that shortly after EGF stimulation, an endosome-like structure containing the ATTO674N label began to form. This result was the first example of twocolor live cell STED imaging and showed that STED microscopy has a great potential in revealing biologically relevant processes in living cells.

Owing to enhanced optical resolution, STED microscopy allows resolution of single vesicles in cells. Using dual color STED, Dean et al. ${ }^{[115]}$ found that after 15 min treatment of neurons with glycine (to induce neural activity), a vesicular transport protein synaptotagmin IV (syt-IV) was present on vesicles at synapses which are distinct from synaptic vesicles. At the same time, synaptotagmin I (syt-I) was shown to be localized to synaptic vesicles. For these experiments, syt-IV or syt-I were labeled with ATTO565, while synaptophysin (a protein ubiquitous in synaptic vesicles) was stained with the large Stokes shift dye DY-485XL. Excitation of ATTO565 was achieved with a diode laser at $532 \mathrm{~nm}$. DY-485XL was excited with the $470 \mathrm{~nm}$ line from a laser diode source. STED 
beam at $647 \mathrm{~nm}$ was used to deplete fluorescence of both dyes. Fluorescence detection for both dyes was realized in the window 560-600 $\mathrm{nm}$.

In another report, ${ }^{[116]}$ two-color super-resolution STED microscopy was successfully used to assess the distribution of IQ motif containing GTPase activating protein (IQGAP1) and phosphorylated Rho GTPases Rac1 and Cdc42 as well as the change in their expression levels upon stimulation of $P$. aeruginosa cells with $N$-3-oxo-dodecanoyl-L-homoserine lactone (3O-C ${ }_{12}-\mathrm{HSL}$, a signaling molecule). IQGAP1 and Rac1/Cdc42 were immunochemically stained with ATTO647N and the large Stokes shift dye Abberior Star 470SXP, respectively. Images were acquired with a commercially available Leica TCS STED microscope.

Mace et al. ${ }^{[117]}$ revealed, using dual-color STED microscopy, that in natural killer cells NK92, lytic granules (specialized secretory lysosomes secreted by NK cells for the elimination of diseased and tumorigenic targets) are closely associated with F-actin and thus secreted through minimally sized clearances. For imaging, NK cells were fixed, permeabilized and stained for F-actin with phalloidin Alexa Fluor 488. The lytic granule component perforin was labeled with the large Stokes shift dye Pacific orange which was conjugated to the anti-perforin antibody.

In another example, topological mapping of presynaptic proteins with nanoscale resolution at the calyx of Held ${ }^{*}$ was realized by dual-color STED microscopy. ${ }^{[118]}$ Two synaptic protein components, the vesicular glutamate transporter (VGluT1) and synapsin were indirectly labeled with Atto565 and the large Stokes shift dye DY-485XL, respectively, using standard immunohistochemistry protocols. Excitation was performed with pulsed laser irradiation at $470 \mathrm{~nm}$ (for DY-485XL) and $532 \mathrm{~nm}$ (for Atto565). A pulsed STED laser at $647 \mathrm{~nm}$ depleted the fluorescence of both fluorophores. The enhanced resolution provided by STED microscopy revealed synaptic vesicles lacking synapsin which was impossible to demonstrate using conventional confocal microscopy (Figure 40).

\footnotetext{
* Calyx of Held is a large synapse in the mammalian auditory central nervous system.
} 

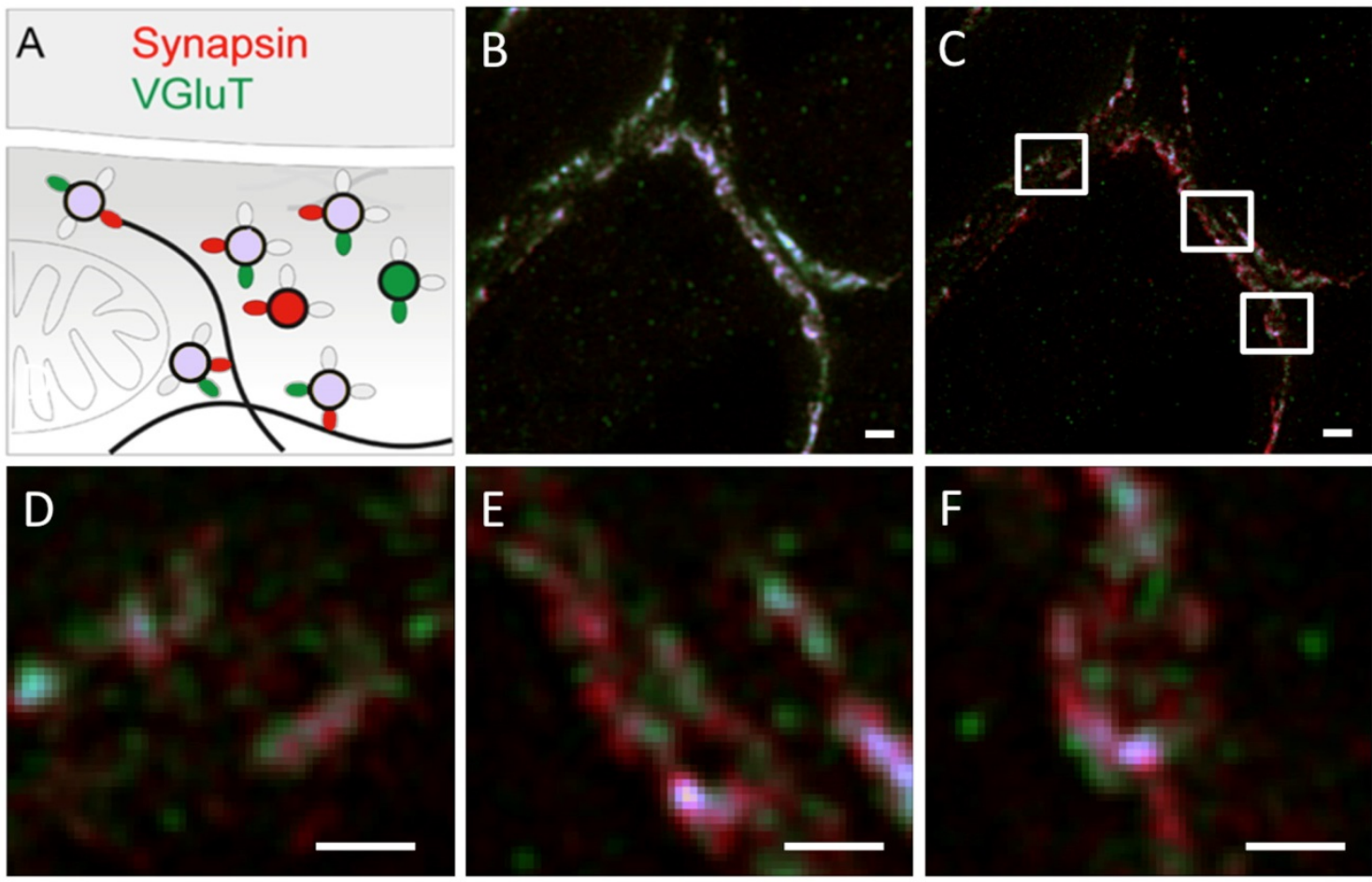

Figure 40 Dual color STED nanoscopy of synapsin (labeled with DY-485XL, shown red) and VGluT1 (labeled with Atto565, shown green) distribution in the calyx of Held. ${ }^{[118]}$ (A) Schematic diagram depicting the hypothesized interplay of synapsin and synaptic vesicles (VGluT1) in a glutamatergic terminal. (B) Dual-color confocal and (C) STED images of synapsin and VGluT1 distribution in the calyx. Scale bars = $1 \mu \mathrm{m}$. (D,E,F) Magnified views of the subregions marked in $\mathrm{C}$ in clockwise order of their appearance. Scale bar $=500 \mathrm{~nm}$.

The lateral resolution provided by STED ( 70 nm) was sufficient to discriminate cis- and trans-Golgi cisternae in a HeLa cell, which are typically separated by at least $200 \mathrm{~nm}$. In a test experiment on two-color STED imaging, transmembrane protein GPP130, which is localized to the cis-Golgi network, was labeled with the large Stokes shift dye Star 470SXP, whereas protein p230, which is found in the trans-Golgi network, was stained with Star 635. ${ }^{[45 b]}$ Another large Stokes shift dye Star 520SXP together with Star 635 was used in a dual-color STED imaging experiment which allowed the assessment of the localization of collagen I (an abundant component of the extracellular matrix) within Golgi ministacks upon treatment with nocodazole and showed that collagen $\mathrm{I}$ is retained at the cis face. ${ }^{[119]}$ This result led the authors to a conclusion that the flow of collagen through the Golgi ministack was inhibited.

Dual-color STED microscopy provided morphological evidence for the organization of anoctamins (voltage sensitive calcium-activated chloride channels) and cyclic nucleotidegated (CNG) channels in distinct domains, thus indicating the existence of signaling 
microdomains in cilia of the olfactory sensory neuron (OSN). ${ }^{[120]}$ In addition, the staining patterns for anoctamins ANO2 and ANO6 observed in high-resolved STED images supported the idea that ANO2 and ANO6 form oligomeric complexes in olfactory cilia. Large Stokes shift dye V500 (BD Horizon) was used for staining tubulin, whereas anoctamins and CNG channels were immunochemically stained with Oregon Green 488 - a conventional dye with a small Stokes shift. Images were acquired using two excitation sources at $458 \mathrm{~nm}$ (for V500) and $514 \mathrm{~nm}$ (for Oregon Green 488) and one STED laser (592 nm). V500 and Oregon Green 488 emission was detected at 465-500 and 540-585 nm, respectively.

The large Stokes shift dyes Star 440SXP and Star 520SXP (Abberior) were utilized in colocalization studies on Norbin (neuron-specific cytosolic protein which interacts with the metabotropic glutamate receptor) and postsynaptic density protein 95 (PSD-95) in neurons. ${ }^{[121]}$ Neuron spines have a submicrometer size, and therefore all constituting proteins appear as more or less colocalized in a confocal microscope. Using super-resolution microscopy methods - 3D-SIM and STED, authors showed that Norbin associates with actin rather than with PSD-95 in dendritic spines.

In a study on the function of the Arf1/COPI protein machinery at cellular lipid droplets (LDs) in mammalian NRK cells, two-color STED microscopy revealed that $\beta$ 'COP (a component of COPI machinery) localize to the lipid droplet surface. ${ }^{[122]}$ For this study, $\beta$ 'COP and the LD marker perilipin3 were immunochemically labeled with the large Stokes shift dye Star 470SXP and the conventional dye ATTO647N, respectively. Images were acquired in a custom-built microscope with a STED laser tuned to either 760 or $770 \mathrm{~nm}$ for the depletion. Two pulsed diode laser lasing at 510 and $640 \mathrm{~nm}$ were used for the excitation of Star 470SXP and ATTO647N. Fluorescence detection was realized at 570-616 nm (for Star 470SXP) and 665-705 nm (for ATTO647N).

Using multiple super-resolution imaging techniques, including two-color gated STED (gSTED) microscopy, which provided spatial resolution down to $\sim 50 \mathrm{~nm}$, Johnson et al. ${ }^{[123]}$ found that endogeneous TFG protein tightly colocalized with both inner and outer subuntits of COPII (protein coating a vesicle which transports proteins from the rough endoplasmatic reticulum to the Golgi apparatus). For immunochemical staining, large Stokes shift dyes Star 440SXP and DyLight 488 were used together with small Stokes 
shift dyes Alexa Fluor 488, 568 and 647. Imaging was performed using the commercial Leica TCS STED system. 


\section{Main part}

\subsection{3-Heteroaryl-substituted coumarin dyes}

\subsubsection{Motivation and key structural elements}

Coumarins are unique among other fluorophores with large Stokes shifts caused by photoinduced ICT. Many coumarins combine moderately high extinction coefficients (20 000-50 000 and large Stokes shifts with good fluorescence quantum yields in polar protic solvents. The synthesis of coumarins has been pioneered long ago beginning with the work of Perkin ${ }^{[124]}$ published in 1868. Modern catalytic methods involving C-H activation $^{[125]}$ represent one of the recent highlights in this area. Most of these synthetic methods are simple, rather straightforward, and require available starting materials and a relatively small number of synthetic steps.

In all coumarin dyes presented in this work, $7-N, N$-dialkylaminocoumarin $\left(\lambda_{\mathrm{abs}, \max } / \lambda_{\mathrm{em}, \max }\right.$ $=380 / 460 \mathrm{~nm}$ in $\mathrm{MeOH}$ for 7-N,N-diethylaminocoumarin) was chosen as a starting scaffold for the design of new fluorescent dyes with large Stokes shift. The introduction of a 2,2,4-trimethyl-1,2-dihydropyridine ring provides a small red-shift of 5-10 nm, ${ }^{\text {[45a] }}$ rigidizes the dye skeleton, improves the photostability and anchors the 7-amino group

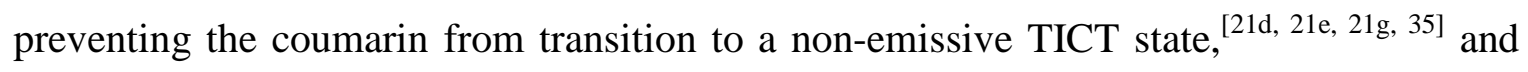
thus enhancing the fluorescence QY in polar solvents. The methyl group at the double bond can be easily modified to introduce various polar groups, such as hydroxyl, sulfonate, phosphonate or phosphate. These groups increase the solubility in aq. media and prevent the fluorophores from aggregation, thus improving the fluorescence QY. At the same time, they are known to have only a minor effect on the positions of absorption and emission maxima.

Unlike alkylsulfonic acids, monoalkyl phosphates and alkylphosphonic acids are dibasic acids. Therefore, upon dissociation they give doubly charged anions which are less prone to aggregation and non-specific binding than the monocharged sulfonates. Comparison of $p K_{a}{ }^{1}$ and $p K_{a}{ }^{2}$ values for monoalkyl phosphates vs. alkylphosphonic acids (1.54-1.80 and $6.31-6.84$ vs. $2.33-2.74^{[126]}$ and $7.76-8.48,{ }^{[127]}$ respectively) leads to a conclusion that at physiological $\mathrm{pH}$ of 7.3-7.4 the former compounds are better ionized (and their aggrega- 
tion is suppressed more efficiently). Due to the double negative charge, monoalkyl phosphates are resistant to nucleophilic attacks and, in particular, to basic hydrolysis. Taking into account all these pieces of information we chose the monophosphate ester as a polar solubilizing unit for the fluorescent dyes presented in this work.

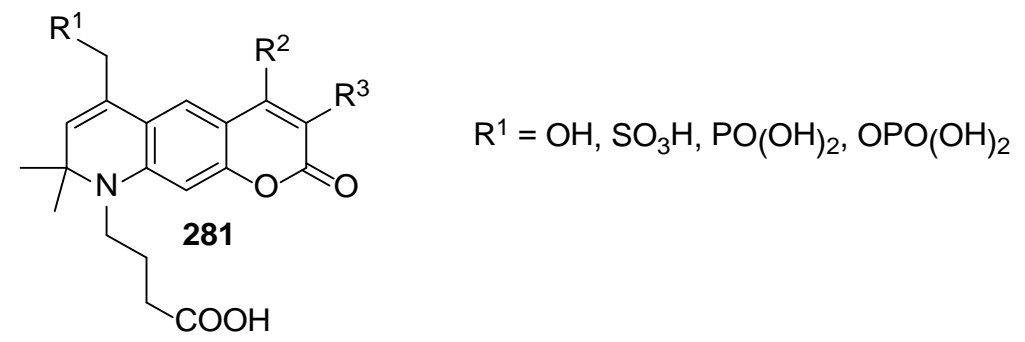

Figure 41 Coumarins with the 2,2,4-trimethyl-1,2-dihydropyridine fragment and various polar groups.

The carboxylic acid residue in coumarin 281 (see Figure 41) is required for bioconjugation. It can be activated by formation of an amino-reactive ester (e.g. an $N$-hydroxysuccinimidyl ester), and used in the reaction with biomolecules having free amino groups (e.g. proteins). The carboxylic group may be attached to the nitrogen via a linker with variable length. A linker having three $\mathrm{CH}_{2}$ groups was found to provide better hydrolytic stability of $N$-hydroxysuccinimidyl esters than a shorter linker derived from propanoic acid. ${ }^{[111 b]}$

The substituents $\mathrm{R}^{2}$ and $\mathrm{R}^{3}$ determine the photophysical properties of the coumarin fluorophore. They can be varied to a great extent. It is known that electron-acceptor groups at C-3 and/or C-4 of the coumarin fragment facilitate the degree of ICT and increase "push-pull" effects in the fluorophore. Therefore, these groups determine the positions of absorption and emission bands, and can shift them towards the red end of the visible spectrum.
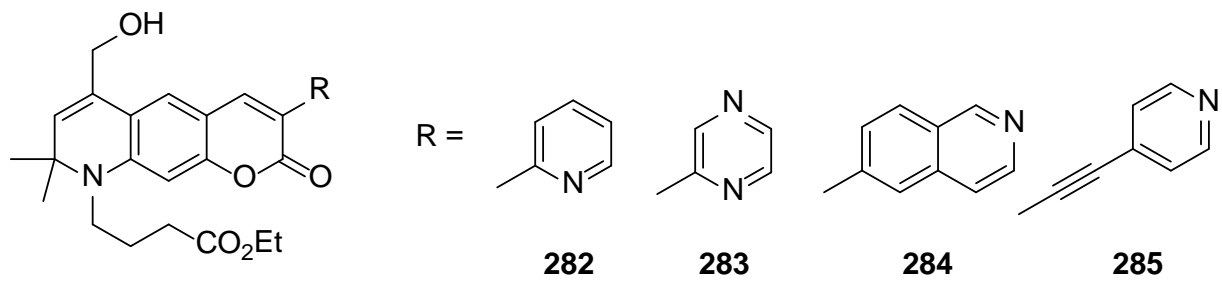

Figure 42 Model coumarins 282-285 with a variable electron-acceptor group at C-3. 
7-Amino-3-phenylcoumarins have an attractive combination of large Stokes shifts and acceptable QYs in polar solvents. ${ }^{[42]}$ However, their absorption bands are found in the violet region of the visible spectrum. One can expect that the introduction of a strong electron-withdrawing substituent on the phenyl ring could provide red-shifts in absorption and emission bands. Many electron-withdrawing groups are available, and their acceptor properties were quantified as $\sigma$-constants in the Hammett equation. In this regard, the nitro group represents an interesting candidate as it is relatively easy to introduce, and it has one of the largest values of the $\sigma$-constant $(0.78) .{ }^{[128]}$ Unfortunately, fluorophores containing the nitro group often display very poor emission efficiency in polar solvents (probably, due to formation of the non-emissive TICT state). On the other hand, it is known that many properties of nitrobenzene are similar to those of pyridine. Due to the larger electronegativity of the nitrogen atom (in comparison to the carbon) and the inability of the lone electron pair to participate in delocalization, pyridine represents a $\pi$ deficient heterocycle, and 2-pyridyl and 4-pyridyl groups exhibit strong $-I$ and $-M$ effects. Indeed, an introduction of 2- or 4-pyridyl groups at C-3 of the 7- $N, N$-dialkylaminocoumarin scaffold provided a bathochromic shift of $\sim 20 \mathrm{~nm}$ (in EtOH) in comparison with analogous 3-phenyl-substituted coumarins. ${ }^{[21 f]}$ The pyrazinyl group has an additional nitrogen atom in the cycle which further increases the $\pi$-deficiency. The quinolyl and isoquinolinyl groups provide an extension of the conjugation chain and can cause further bathochromic and bathofluoric shifts. We expected that a 4-pyridylethynyl group would also provide an extension of the conjugation chain and a small $-M$-effect enhanced by the neighboring $\pi$-deficient pyridine ring. Thus, at the beginning of this work, we decided to synthesize coumarins 282-285 without phosphate groups (see Figure 42). This would allow us to evaluate and compare the properties of the said groups, and probably obtain dyes with large Stokes shifts and good fluorescence QYs. In view of the apparent lack of photostable and bright fluorescent dyes applicable in two-color STED imaging (only a few fluorescent dyes were successfully applied in two-color STED: DY-480XL, DY485XL, Chromeo 494, Abberior STAR 440SXP), the design of new dyes with large Stokes shift is particularly important. Moreover, live-cell imaging requires more cellpermeable dyes for all spectral regions. 


\subsubsection{Synthesis of model compounds}

The universal coumarin precursor $\mathbf{2 8 9}$ was prepared in five steps starting from commercially available $m$-aminophenol 286-H (see Scheme 9). First, the phenolic residue in compound 286-H was protected with t-butyldimethylsilyl chloride (TBDMSCl) using the standard protocol. The protected TBDMS derivative 286-TBDMS was then converted to 2,2,4-trimethyl-1,2-dihydroquinoline $\mathbf{2 8 7}$ using a modified Skraup reaction with acetone catalyzed by anhydrous $\mathrm{Yb}(\mathrm{OTf})_{3} \cdot{ }^{[129]}$ The anilinic nitrogen in compound 287 is hindered by adjacent methyl groups, and its alkylation required relatively harsh conditions. Nevertheless, reaction of ethyl 4-iodobutanoate with 287 in neat DIEA at $110{ }^{\circ} \mathrm{C}$ afforded the ester 288-TBDMS after $48 \mathrm{~h}$ in an excellent yield. Next, the hydroxyl group in 288TBDMS was deprotected with tetrabutylammonim fluoride in THF, and the resulting phenol 288-H was then formylated according to Vilsmeier-Haack to furnish the silicic aldehyde 289.

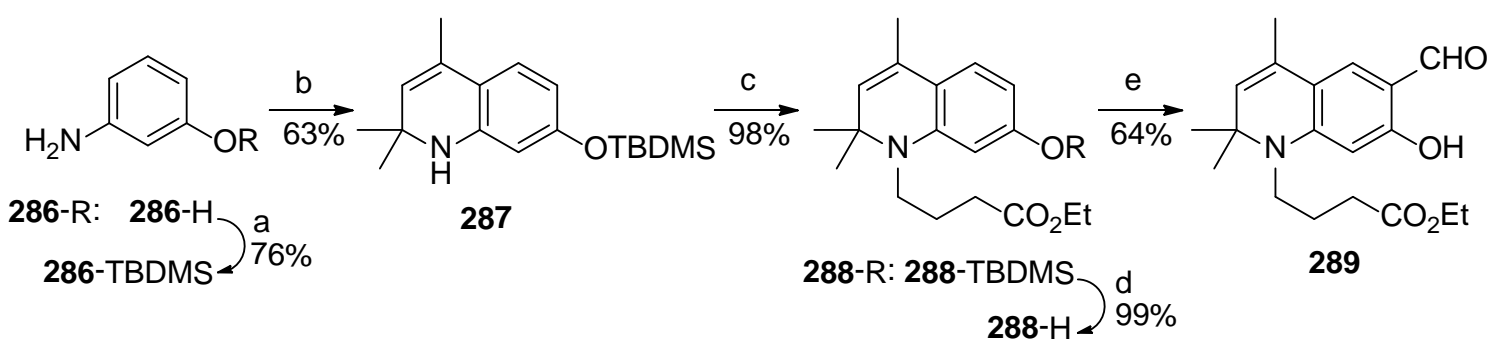

Scheme 9 Synthesis of the universal precursor 289: a) TBDMSCl, imidazole, DMF, 0 to $55^{\circ} \mathrm{C}, 2 \mathrm{~h}$; b) acetone, $\mathrm{Yb}(\mathrm{OTf})_{3}$, r.t., $16 \mathrm{~h}$; c) ethyl 4-iodobutanoate, DIEA, $110^{\circ} \mathrm{C}, 48 \mathrm{~h}$; d) TBAF· $3 \mathrm{H}_{2} \mathrm{O}$, THF, $0{ }^{\circ} \mathrm{C}, 5$ min; e) $\mathrm{POCl}_{3}$, DMF, $60{ }^{\circ} \mathrm{C}, 1.5 \mathrm{~h}$.

In order to obtain the target dyes 282-285 using precursor $\mathbf{2 8 9}$, two synthetic approaches were employed. According to the first approach, the coumarin ring was formed in the course of the esterification reaction which involved phenol $\mathbf{2 8 9}$ and a heteroaryl acetic acid followed by a Knoevenagel condensation (route $c$ in Scheme 10). 2-Pyridylacetic acid is commercially available. 2-Piperazinylacetic acid $\mathbf{2 9 9}$ was conveniently prepared by lithiation of 2-methylpiperazine 298 with $t$ BuLi followed by quenching the resulting lithiated derivative with dry ice. In initial experiments we found out that compound 299 being dissolved in ethyl acetate readily loses carbon dioxide, if the solution is evaporated at elevated temperatures $\left(>40{ }^{\circ} \mathrm{C}\right)$. To our delight, when all operations were performed at room temperature or below, the compound 299 could be isolated with a moderate yield. 
This tendency to loose carbon dioxide is even more pronounced in the case of (6methylpyrimid-4-yl)acetic acid: in an attempted synthesis according to the same method, the decarboxylation readily occurred already upon acidification of the reaction mixture (even at low temperatures).

The second approach employs 3-bromocoumarin 297 and its Pd-catalyzed cross-coupling reactions with 2-(tributylstannyl)pyridine (route $e$ ), boronic ester $\mathbf{3 0 1}$ (route $f$ ) or 4-pyridylacetylene (route $\mathrm{g}$ ). Aryl bromide $\mathbf{2 9 7}$ was obtained from the universal precursor $\mathbf{2 8 9}$ in three steps. First, the coumarin ring was formed in the course of a Wittig reaction of 289 with (ethoxycarbonylmethylene)triphenylphosphorane followed by the cyclization reaction. In initial trials we tried to brominate compound 290, but along with the desired aryl bromide, we also obtained substantial amounts of the corresponding allyl bromide. Therefore, before the bromination, we oxidized the methyl group attached to the $\mathrm{C}=\mathrm{C}$ bond into a formyl group using $\mathrm{SeO}_{2}$ in dioxane. Final bromination of aldehyde 291 proceeded smoothly and rapidly in acetic acid at room temperature and afforded the required bromocoumarin 297 in a high yield. 

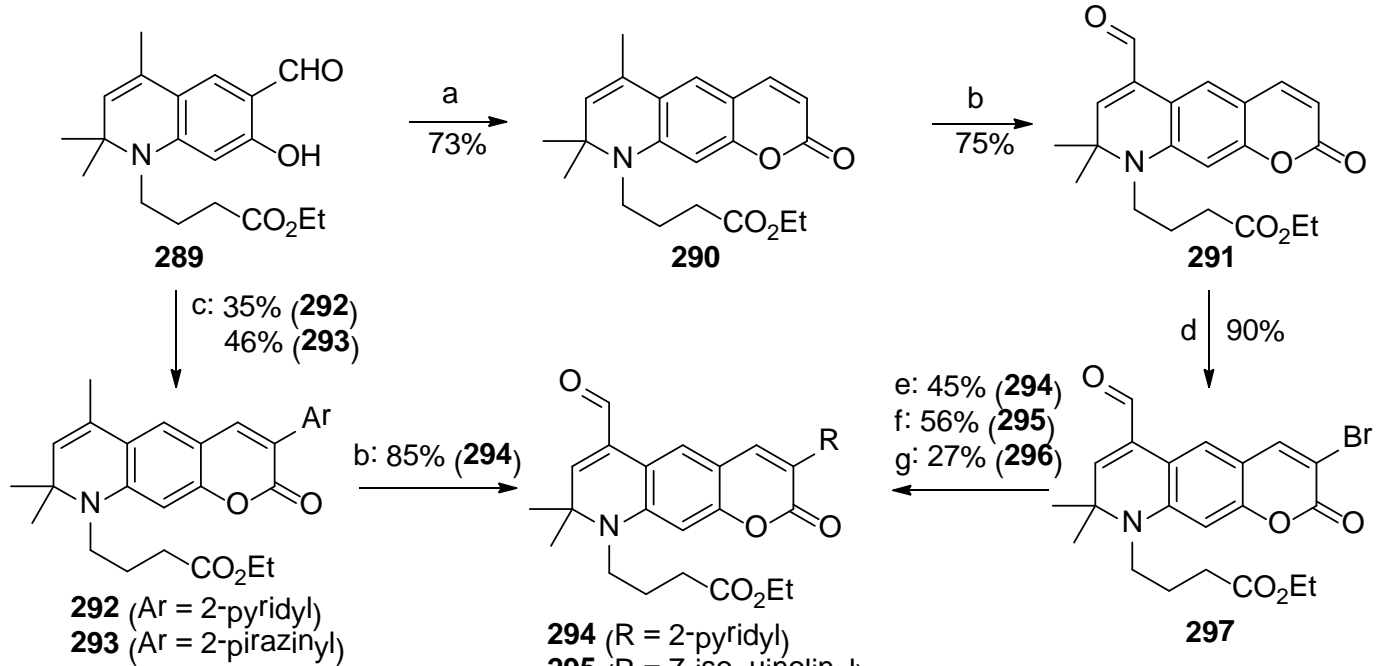

$294(R=2-p y$ ridyl)
$295(R=7$-isoquinolin $y l)$
$296(R=(4-p y r i d y l)$ ethynyl

297
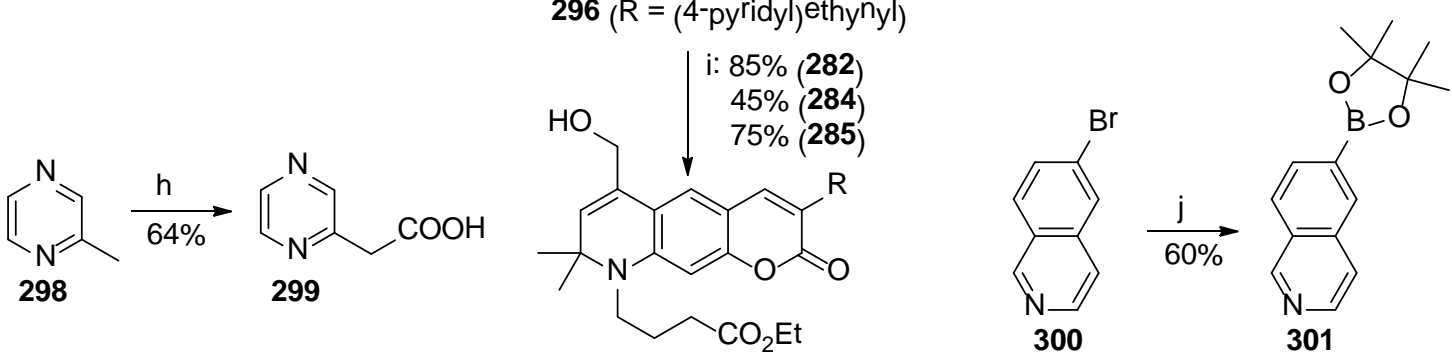

$$
\begin{aligned}
& 282(R=2-p y \text { ridyl }) \\
& 284\left(R=7-i s q_{\text {quinolin }} \text { l }\right) \\
& 285(R=(4-p y \text { ridyl }) \text { ethynyl })
\end{aligned}
$$

Scheme 10 Synthesis of the model 3-substituted coumarins 282, 284 and 285: a) (ethoxycarbonylmethylene)triphenylphosphorane, xylene, $140{ }^{\circ} \mathrm{C}, 3 \mathrm{~h}$; b) $\mathrm{SeO}_{2}$, dioxane, reflux, $2 \mathrm{~h}$; c) heteroarylacetic acid, EDC $\cdot \mathrm{HCl}, \mathrm{NEt}_{3}$, DMAP, $\mathrm{CH}_{2} \mathrm{Cl}_{2} / \mathrm{DMF}, 30{ }^{\circ} \mathrm{C}, 20$ h; d) $\mathrm{Br}_{2}$, $\mathrm{AcOH}$, r.t., 15 min; e) 2-(tributylstannyl)pyridine, $\mathrm{Pd}\left(\mathrm{PPh}_{3}\right)_{4}$, dioxane, $110{ }^{\circ} \mathrm{C}$, overnight; f) 301, $\mathrm{Pd}\left(\mathrm{PPh}_{3}\right)_{4}, 2 \mathrm{M}$ aq. $\mathrm{Na}_{2} \mathrm{CO}_{3}$, toluene, $110{ }^{\circ} \mathrm{C}$, overnight; g) 4-ethynylpyridine, $\mathrm{Pd}_{2}(\mathrm{dba})_{3}, \mathrm{P}(\mathrm{BBu})_{3}$, CuI, $\mathrm{NEt}_{3}$, THF, r.t., 8 h; h) MeI, MeCN, $70{ }^{\circ} \mathrm{C}$, overnight; i) $\mathrm{NaBH}_{4}, \mathrm{CeCl}_{3}$, THF/MeOH, $0{ }^{\circ} \mathrm{C}, 15 \mathrm{~min}$; k) LDA, THF, -78 ${ }^{\circ} \mathrm{C}, 1 \mathrm{~h}$; then $\mathrm{CO}_{2},-78^{\circ} \mathrm{C} \rightarrow$ r.t., overnight; l) $t \mathrm{BuLi}$, THF, $-78{ }^{\circ} \mathrm{C}, 20 \mathrm{~min}$; then $\mathrm{B}(\mathrm{OiPr})_{3},-78{ }^{\circ} \mathrm{C} \rightarrow$ r.t., overnight; then AcOH, pinacol, r.t., 2 h.

Having coumarin 297 at hand, we tried to perform Stille, Suzuki and Sonogashira reactions and obtain compounds 294-296 with 2-pyridyl, 7-isoquinolinyl and (4pyridyl)ethynyl groups, respectively. The Stille and Suzuki coupling reactions employed $\mathrm{Pd}\left(\mathrm{PPh}_{3}\right)_{4}$ as a catalyst and proceeded in a clean fashion, although the preparative yields did not exceed 56\%. In contrast, a Sonogashira reaction in the presence of $\mathrm{Pd}\left(\mathrm{PPh}_{3}\right)_{4}$ and CuI did not occur at all. When $\mathrm{Pd}_{2}(\mathrm{dba})_{3}$ and $\mathrm{P}(t \mathrm{Bu})_{3}$ were used to form a more active Pdcatalyst in the reaction mixture, a very low conversion of $\mathbf{2 9 7}$ was observed, even when the whole amount of alkyne was consumed. Only with a two-fold excess of 4ethynylpyridine which was added to the reaction mixture in four portions over an eight- 
hour interval, we obtained a modest yield (27\%) of 296 and observed a 33\% conversion of the starting material 297. Pinacol boronate 301 was prepared from 6-bromoisoquinoline $\mathbf{3 0 0}$ in the course of a lithium-halogen exchange followed by a reaction of the resulting organolithium compound with $\mathrm{B}(\mathrm{OiPr})_{3}$, and a transesterification of the intermediate diisopropyl boronate with pinacol in the presence of acetic acid. 4-Ethynylpyridine was kindly provided by Dr. S. Nizamov.

Reduction of conjugated aldehydes 294-296 to the corresponding allylic alcohols can be complicated by 1,4-reduction. In order to minimize the amount of undesired products resulting from 1,4-reduction, we utilized Luche's reduction procedure. ${ }^{[130]}$ According to it, $\mathrm{NaBH}_{4}$ in the presence of $\mathrm{CeCl}_{3}$ reduced compounds 294-296 in the 1,2-fashion and afforded model coumarins 282,284 and 285.

\subsubsection{Spectral properties of model coumarin dyes}

Table 9 presents the most important photophysical properties of compounds 282, 284, 285 and 293. Thus, coumarin 293 with a pyrazinyl group at C-3 has the absorption maximum at $451 \mathrm{~nm}$, when dissolved in $\mathrm{CH}_{2} \mathrm{Cl}_{2}$ or $\mathrm{MeOH}$. Upon excitation, it emits green light with the maximum at 506 (in $\mathrm{CH}_{2} \mathrm{Cl}_{2}$ ) or $535 \mathrm{~nm}$ (in $\mathrm{MeOH}$ ). The observed bathofluoric shift of $29 \mathrm{~nm}$ upon transition from $\mathrm{CH}_{2} \mathrm{Cl}_{2}$ to $\mathrm{MeOH}$ corresponds to the general trend exhibited by many other coumarin dyes. ${ }^{[21 \mathrm{e}]}$ This behavior is attributed to a better stabilization of the excited state $S_{1}$ by a more polar solvent. The red-shift of the fluorescence maximum is also accompanied by a significant decrease of fluorescence quantum yield from 0.78 in $\mathrm{CH}_{2} \mathrm{Cl}_{2}$ to 0.08 in $\mathrm{MeOH}$. One possible explanation of this phenomenon could be a photoinduced electron transfer (PET) accelerated in polar $\mathrm{MeOH}$. In the course of this process, in the excited state $S_{1}$, an electron from one of the lone pairs of the pyrazine moiety fills the vacant place in the half-occupied HOMO of the fluorophore, and thus makes the emission impossible. This kind of electron transfer can take place only if the molecular orbital, which contains the lone pair electrons, has a higher energy than the HOMO of the fluorophore in the ground state. A comparison of the calculated energies for the highest-energy orbital, which is occupied by the electron lone-pair, in nitrogen heterocycles gave the following values for pyrazine and pyridine: $-10.25 \mathrm{eV}$ and -9.93 $\mathrm{eV}$, respectively (by AM1 method). ${ }^{[131]}$ These values were also in accordance with experimental $p K_{\mathrm{a}}$-values (0.4 and 5.2), ${ }^{[132]}$ and revealed that a PET from the nitrogen atom in 
the pyridine moiety should proceed more readily. In contrast to this conclusion, coumarin 282 with a 2-pyridyl fragment shows no sign of emission quenching and displays a high fluorescence QY in $\mathrm{MeOH}$. Another explanation of the fluorescence quenching might be formation of a hydrogen bond between the lone electron pairs of the 2-pyrazinyl moiety in 293 in the excited state $\mathrm{S}_{1}$ and a molecule of $\mathrm{MeOH}$. This phenomenon may create an additional non-radiative decay path for compound 293. If this explanation were true, it is still unclear why the same interaction does not lead to the fluorescence quenching in the case of compound 282.

The positions of absorption and emission maxima for compound $\mathbf{2 8 2}$ were found to be close to those of the analogous dye $\mathbf{5 6}$ possessing the julolidine fragment. ${ }^{[21 f]}$ The transition from a 2-pyridyl to a 7-isoquinolinyl substituent (compound 284) did not influence the shape and the position of the long-wavelength absorption maximum, but provided a small bathofluoric shift of $13 \mathrm{~nm}$, thus increasing the observed Stokes shift from 67 to 80 nm. Coumarin 285 with a (4-pyridyl)ethynyl substituent at C-3 had the most red-shifted absorption maximum in this series, but its value of Stokes shift (54 nm) turned out to be rather low: the emission maximum was centered at $511 \mathrm{~nm}$. All coumarins 282, 284-285 displayed high fluorescence QYs in $\mathrm{MeOH}$ in the range of $0.62-0.72$. Due to these attractive spectral properties model coumarins $\mathbf{2 8 2}$, 284-285 were chosen for further development (phosphorylation).

Table 9 Spectral properties of model coumarins 282, 284, 285, 293 and phosphorylated coumarin 43 in various solvents.

\begin{tabular}{ccccccc}
\hline Compound & $\lambda_{\text {abs,max }}, \mathrm{nm}$ & $\varepsilon, \mathrm{M}^{-1} \cdot \mathrm{cm}^{-1}$ & $\lambda_{\mathrm{em}, \max }, \mathrm{nm}$ & $\Delta \lambda, \mathrm{nm}$ & $\Phi_{\mathrm{fl}}$ & Solvent \\
\hline $\mathbf{2 8 2}$ & 431 & 32000 & 498 & 67 & 0.67 & $\mathrm{MeOH}$ \\
$\mathbf{2 8 4}$ & 431 & 41100 & 511 & 80 & 0.72 & $\mathrm{MeOH}$ \\
$\mathbf{2 8 5}$ & 457 & 44200 & 511 & 54 & 0.62 & $\mathrm{MeOH}^{2}$ \\
$\mathbf{2 9 3}$ & 451 & 39200 & 506 & 55 & 0.74 & $\mathrm{CH}_{2} \mathrm{Cl}_{2}$ \\
$\mathbf{2 9 3}$ & 451 & 39600 & 535 & 84 & 0.08 & $\mathrm{MeOH}^{2}$ \\
$\mathbf{3 0 8}$ & 432 & 20400 & 512 & 80 & 0.81 & $\mathrm{PBS}_{7.4}$ \\
\hline
\end{tabular}




\subsubsection{Synthesis of water-soluble coumarins with a phosphate group}

For the synthesis of 3-heteroaryl-substituted coumarins with a phosphorylated $\mathrm{CH}_{2} \mathrm{OH}$ group we used the route presented in Scheme 11. Aldehyde 297 with the bromine substituent at C-3 was reduced using Luche's reduction method. ${ }^{[130]}$ According to the known procedure, ${ }^{[133]}$ alcohol $\mathbf{3 0 2}$ readily reacted with di-tert-butyl $N, N$-diisopropylphosphoramidite in the presence of $1 \mathrm{H}$-tetrazole and gave the intermediate phosphite which was not isolated and directly oxidized in the reaction mixture with $m C P B A$ providing the phosphate $\mathbf{3 0 3}$ in a high preparative yield (87\% in two synthetic steps).

At this point, we tried to improve the synthetic yield of the cross-coupling reactions of bromide 303 in comparison with the analogous reactions of 297 (see section 2.1.2) and, at the same time, to reduce the possibility of any side reactions at high temperatures. For this purpose, we attempted a cross-coupling reaction of coumarin 303 with 2-(tributylstannyl)pyridine at room temperature in the presence of a highly active Pd catalyst prepared in situ from $\mathrm{Pd}(\mathrm{OAc})_{2}$ and $\mathrm{P}(\mathrm{Bu})_{3}$. Unfortunately, at room temperature, this reaction did not proceed at all. At $110{ }^{\circ} \mathrm{C}$, the starting bromide $\mathbf{3 0 3}$ had disappeared completely in the reaction mixture after $2 \mathrm{~h}$. However, the coupling product - coumarin 304 with the 3-(2-pyridyl) substituent - was isolated with an only moderate yield of 56\%.

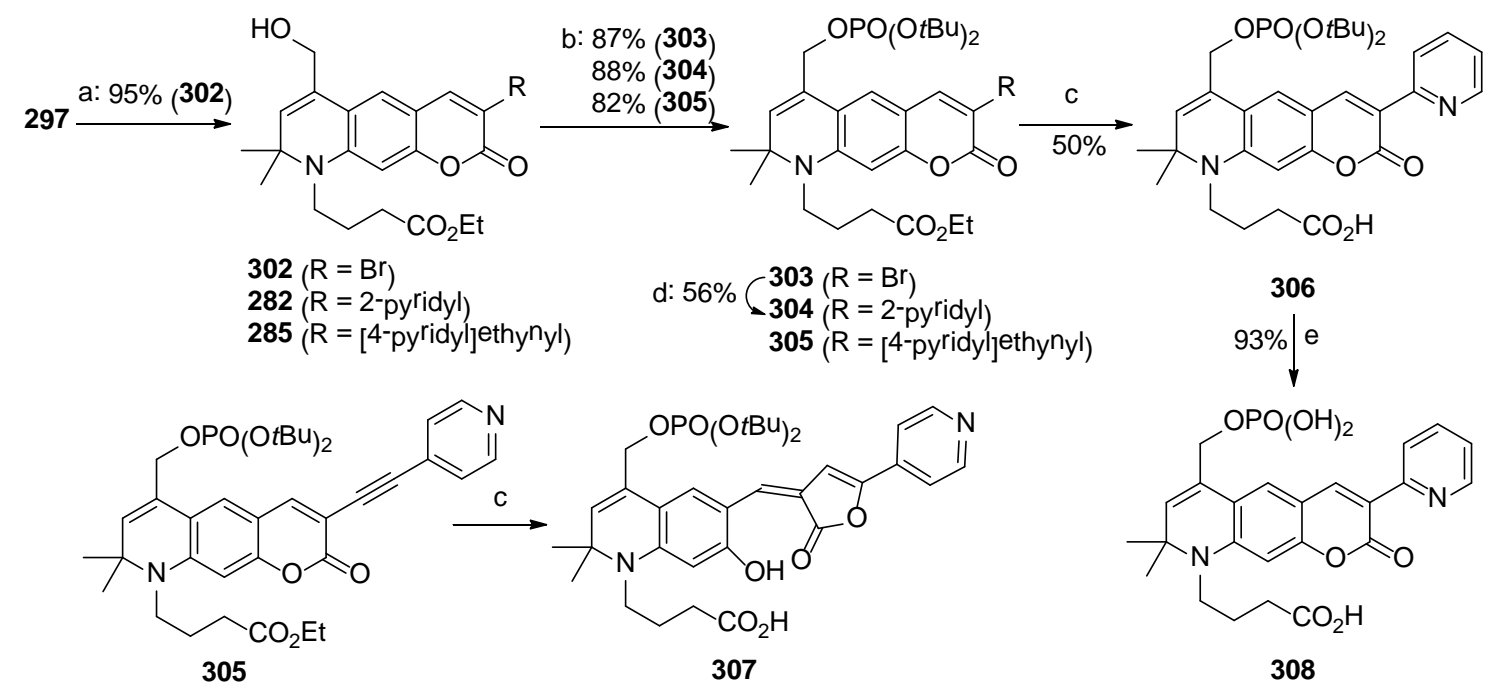

Scheme 11 Synthesis of water-soluble coumarins with a primary phosphate group: a) $\mathrm{NaBH}_{4}, \mathrm{CeCl}_{3}$, THF/MeOH, $0{ }^{\circ} \mathrm{C}, 15 \mathrm{~min}$; b) $i \mathrm{Pr}_{2} \mathrm{NP}(\mathrm{OtBu})_{2}, 1 \mathrm{H}$-tetrazole, $\mathrm{CH}_{2} \mathrm{Cl}_{2}, 40{ }^{\circ} \mathrm{C}, 1 \mathrm{~h}$; then $\mathrm{mCPBA}, 0{ }^{\circ} \mathrm{C}, 15$ min; c) aq. $\mathrm{NaOH}, \mathrm{THF} / \mathrm{MeOH}$, r.t., overnight; d) 2-(tributylstannyl)pyridine, $\mathrm{Pd}(\mathrm{OAc})_{2}, \mathrm{P}(t \mathrm{Bu})_{3}$, toluene, $110{ }^{\circ} \mathrm{C}, 2 \mathrm{~h}$; e) TFA, $\mathrm{CH}_{2} \mathrm{Cl}_{2}$, r.t., 1 h. 
The same phosphorylation procedure ${ }^{[133]}$ was applied to coumarins 282 and 285 and provided phosphates 304 and 305 in good yields of 88 and 82\%, respectively. Compounds 304 and 305 already have all the functionalities required for the new 3-heteroarylcoumarin dyes. The carboxylic acid and phosphate groups are orthogonally protected as ethyl and tert-butyl esters, respectively. They should be removed in the next synthetic steps. Isolation and handling of free phoshates involves aqueous solutions and reversephase chromatography. Thus, we decided to cleave the ethyl ester function first. Saponification of ester 304 afforded carboxylic acid 306 with a rather moderate yield of 50\%. A possible side reaction, which may decrease the yield of the desired product, could involve the opening of the coumarin ring followed by the formation of a salt of cis-coumarinic acid. This process is known to be reversible, and the cyclization to the starting coumarin takes place upon acidification. However, under prolonged action of a base, the cis-coumarinate isomerizes to the trans-isomer which cannot cyclize back to the starting coumarin upon acidification. ${ }^{[33]}$ Both tert-butyl groups in compound 306 were easily removed upon treatment with trifluoroacetic acid, and the target dye $\mathbf{3 0 8}$ was isolated in an excellent yield.

Unexpectedly, all attempts to saponify the ester function in coumarin 305 having a (4pyridyl)ethynyl substituent did not result in the required product. After addition of $1 \mathrm{M}$ $\mathrm{NaOH}$ to a solution of ester $\mathbf{3 0 5}$ in a THF/MeOH mixture, the reaction mixture immediately changed its color from yellow-green (characteristic for coumarins 282, 284-285, 304 and 305) to dark-red. Thin-layer chromatography showed the presence of a redcolored and weakly fluorescent compound as a main product. Although this product was not isolated, we suspect that the basic hydrolysis led to the opening of the coumarin ring, and the resulting carboxylate anion attacked the triple bond which is activated towards a nucleophilic addition by the presence of the neighboring pyridine ring. If this is true, then compound 307 with a five-membered lactone ring and an extended $\pi$-conjugated system was formed, and this can explain the appearance of the intense red color.

\subsubsection{Spectral properties and imaging performance of coumarin 308}

As expected, the phosphate residue in compound $\mathbf{3 0 8}$ provided solubility in aqueous PBS buffer (pH 7.4) and a large fluorescence quantum yield. Upon dissociation of the phos- 
phate group, dye molecules gain negative charges. Due to electrostatic repulsion, the resulting anions are much less prone to aggregation which is believed to be responsible for the reduced fluorescence quantum yields observed for the hydrophobic dyes in polar solvents. ${ }^{[134]}$ In coumarin 308, the introduction of the phosphate group and the transition from $\mathrm{MeOH}$ to the aqueous buffer did not change the position of the absorption maximum (see Table 9). However, the value of molar absorptivity became somewhat smaller in comparison to the parent dye 282. The position of the fluorescence maximum of phosphorylated coumarin 308 shifted towards longer wavelengths by $14 \mathrm{~nm}$ and resulted in a larger Stokes shift of $80 \mathrm{~nm}$.

To prepare bioconjugates for immunochemical staining, the dye must be attached to an antibody or another target molecule by means of a strong covalent bond. This bond must be stable enough and withstand such operations as chromatography, washing, permeabilization, fixation and mounting (in the case of immunochemical staining). In this respect, a peptide bond between the carboxylic group of the dye and a primary amino group represents a viable option. If we consider the labeling of antibodies, modification of lysine residues, due to their relatively high abundance in mammalian proteins, may provide high degrees of labeling and improve the fluorescence signal. For the formation of a peptide bond in aqueous medium, the carboxylic group of any dye (and coumarin 308) must be converted to an active ester, such as $N$-hydroxysuccinimidyl, 4-sulfotetrafluorophenyl, tetrafluorophenyl or sulfodichlorophenyl ester, or to a carbonyl azide. ${ }^{[135]} \mathrm{N}$-Hydroxysuccinimidyl esters provide a good balance between the reactivity towards amines and hydrolytic stability under basic conditions $(\mathrm{pH}>7-8)$. Under these conditions the aliphatic amines cannot be protonated, and therefore, they readily undergo acylation.

The $N$-hydroxysuccinimidyl ester $\mathbf{3 0 9}$ was prepared from $N$-hydroxysuccinimide and coumarin 308 which was activated with HATU in the presence of $\mathrm{NEt}_{3}$ (Scheme 12). In order to reach complete conversion of the starting dye, the long reaction time (16 h) and large excesses of $N$-hydroxysuccinimide, HATU and $\mathrm{NEt}_{3}$ were used. However, the overall reaction was not clean, and the preparative yield of 309 was only modest (42\%). This result may be explained with competitive esterification of the phosphate group. ${ }^{\text {[136] }}$ 


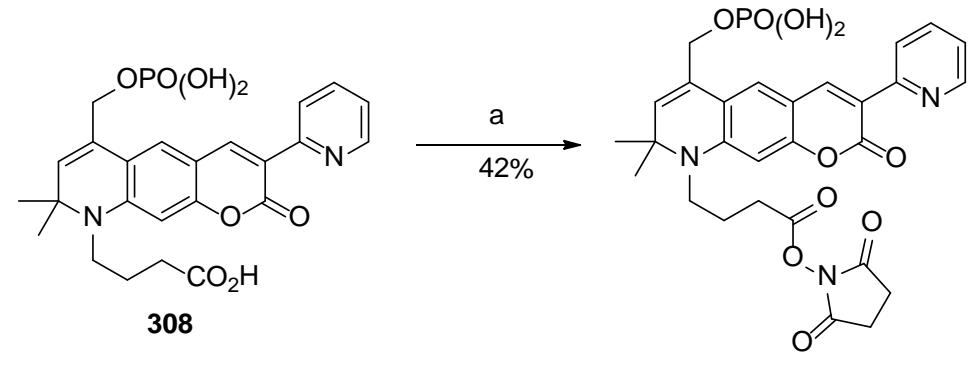

309

Scheme 12 Synthesis of NHS-ester 309: a) $N$-hydroxysuccinimide, HATU, $\mathrm{Et}_{3} \mathrm{~N}$, DMF, r.t., 16 h.

The performance of the phosphorylated coumarin $\mathbf{3 0 8}$ in optical microscopy is illustrated in Figure 43. This figure shows the images of microtubules immunolabeled with dye $\mathbf{3 0 8}$ bound to secondary antibodies. Pictures in Figure 43 represent confocal and STED images obtained in a fluorescence microscope. The superresolution STED image was acquired using a $405 \mathrm{~nm}$ laser as the excitation source, the STED beam applied at $590 \mathrm{~nm}$ and the detection window between 510 and $560 \mathrm{~nm}$. Under these conditions, the new dye provided an optical resolution of ca. $50 \mathrm{~nm}$. Owing to its large Stokes shift (80 nm) and the emission band which overlaps with the emission of dyes Alexa Fluor 488 and Oregon Green (with small Stokes shifts), phosphorylated coumarin 308 can be successfully employed in multicolor STED experiments. Similar dyes were already used in singlecolor STED microscopy and showed sub-diffractional optical resolution of approximately $70 \mathrm{~nm} \cdot{ }^{[137]}$ For realizing a two-color STED imaging using one of these dyes and coumarin 308, it is sufficient to add to the optical setup a second laser for the excitation (at $488 \mathrm{~nm}$ ) of Alexa Fluor 488 or Oregon Green dyes. 

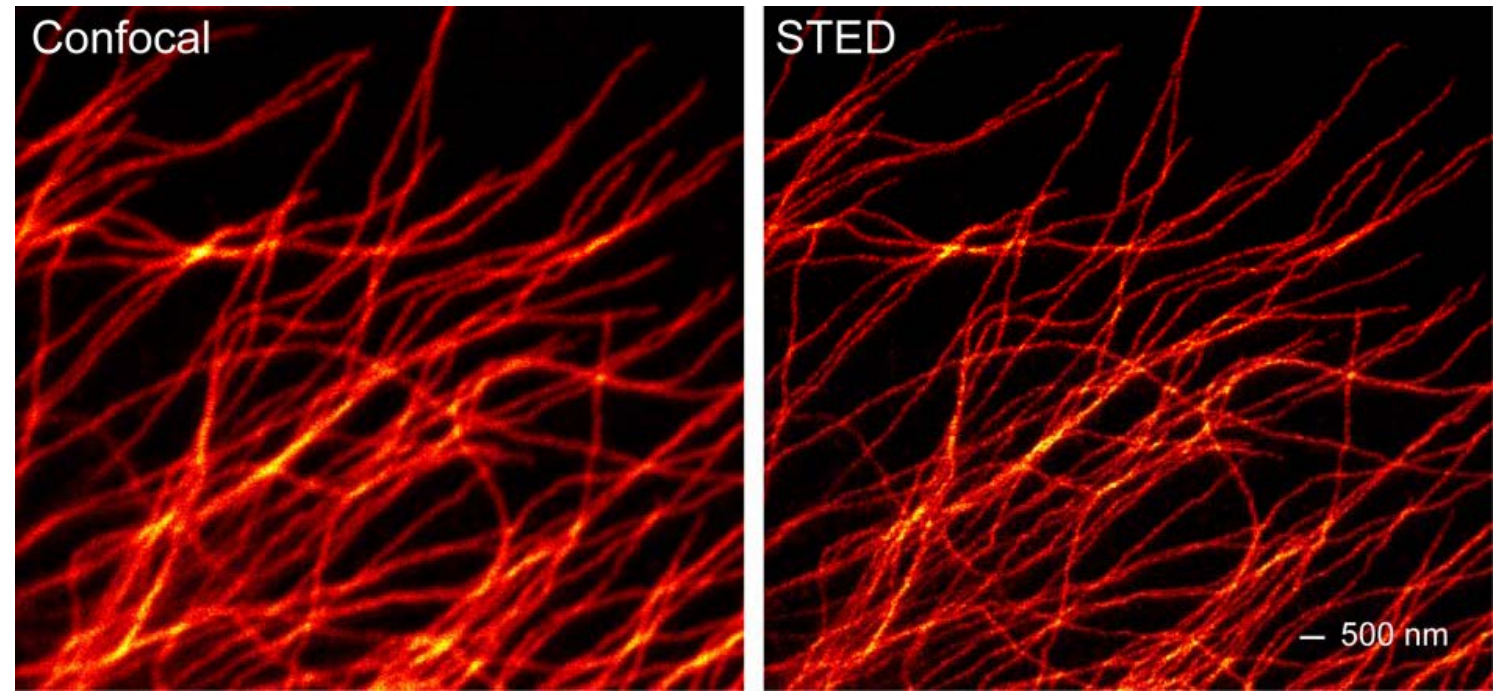

Figure 43 Confocal and STED microscopy images of microtubules stained with coumarin 308. The mammalian cell was fixed and immunolabeled with a primary and secondary antibody (the latter was conjugated with compound 308). Excitation at $405 \mathrm{~nm}$; detection at 510-560 nm; STED at $590 \mathrm{~nm}$ (STED power ca. $84 \mathrm{~mW}$ at the back focal plane).

\subsubsection{Conclusion and outlook}

New coumarins with 3-heteroaryl and a 3-(4-pyridyl)ethynyl groups have been prepared from the universal precursor 289 using two different synthetic routes. These approaches enabled us to shift the absorption and emission maxima of new compounds towards the red spectral region. Thus, synthesized compounds 282, 284-285, 293 and 308 have absorption and emission maxima in the ranges of 431-457 nm and 498-535 nm, respectively. Coumarin 293 with 3-(2-piperidyl)-group had a low emission efficiency in $\mathrm{MeOH}$, whereas compounds 282, 284-285 and $\mathbf{3 0 8}$ provided good fluorescence QYs and large Stokes shifts. The presence of the primary phosphate group in coumarin $\mathbf{3 0 8}$ provided good solubility and a high fluorescence QY in aqueous media. The confocal and superresolution images of microtubules stained with compound $\mathbf{3 0 8}$ demonstrated good signalto-noise ratio and very good optical resolution ( $\sim 0 \mathrm{~nm}$ under STED conditions). Detection and co-localization of various biological objects can be achieved with dye $\mathbf{3 0 8}$ and a conventional "green” dye with small Stokes shift (for example, Oregon Green 488), using one detection channel and two excitation sources (405 and $488 \mathrm{~nm}$ lasers, respectively). Compounds 284-285 also “deserve” further improvement by the introduction of polar groups, which is expected to increase their solubility in aqueous media, fluorescence QYs and brightness in optical microscopy. 


\subsection{3-Pyridiniumcoumarins}

\subsubsection{Motivation and key structural elements}

As a rule, the neutral electron-withdrawing groups attached to C-3 and/or C-4 of the 7-aminocoumarin fluorophore do not shift the absorption maximum of the dye further than $450 \mathrm{~nm}^{*}$ Compounds 310 and 311 (Abberior Star 470SXP) ${ }^{[45 a]}$ (Figure 44) possess the substituent at C-3 which has a betaine structure with the positively charged nitrogen atom; they absorb in the green region of the visible spectrum with a maximum at about $520 \mathrm{~nm}$. The large bathochromic shift may be explained by strong $-I$ - and $-M$-effects (for 4- $N$-methylpyridinium group $\sigma=2.57^{[138]}$ in the Hammett equation) of the quaternized pyridine ring which is in a direct polar conjugation with the amino group at C-7. As a result, this group facilitates the ICT from the 7-amino group shifting the absorption and emission bands towards the red region of the visible spectrum.

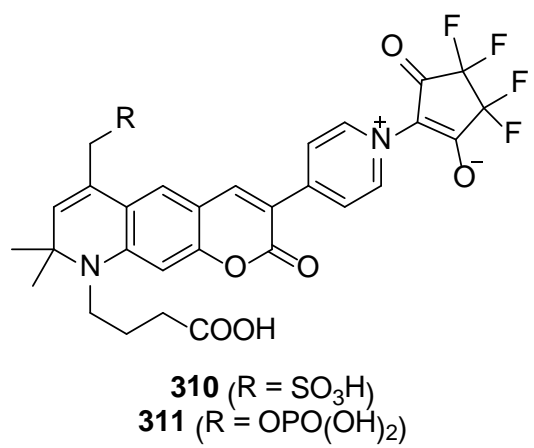

Figure 44 Coumarins 310 and 311.

Despite the attractive spectral properties, coumarins 310 and 311 have very low fluorescence QYs in polar solvents. The presence of the tetrafluorocyclopentadienone fragment which can rotate around the $\mathrm{C}-\mathrm{N}$ bond, thus creating an additional pathway for a nonradiative deactivation of the excited state $S_{1}$, might be responsible for poor emission efficiencies. Commercially available dyes DY-480XL, DY-481XL, DY-485XL, DY-510XL, DY-511XL, DY-520XL, DY-521XL possess a quaternized pyridine ring, attached to the 3-position of the coumarin via a trans-double bond. These dyes have spectral properties

\footnotetext{
* A notable exception is the combination of a $\mathrm{CF}_{3}$-group at $\mathrm{C}-4$ and an (hetero)arylvinyl group at C-3 which is known to produce large bathochromic and bathofluoric shifts. ${ }^{[48]}$
} 
similar to those of $\mathbf{3 1 0}$ and 311, and their QYs have not been disclosed. The other dyes with large Stokes Shift, ATTO 490LS and Chromeo 494, also absorb in the yellow region, but their structures are unknown. Therefore, a direct comparison of their structures and properties with those of compounds 310, 311 and Dyomics dyes is very difficult.

It is known that the boron-fluorine complexes with $N, N$ - or $N, O$-bidentate ligands, such as boron-dipyrromethenes (BODIPYs), ${ }^{[77]}$ boron 2-(2-pyridyl)imidazoles (BOPIMs), ${ }^{[84]}$ and complexes with (2-quinolin-2-yl)phenol, ${ }^{[139]}$ are often fluorescent. Despite the presence of nitrogen atoms with partial positive charges, some of these compounds display high fluorescence QYs even in polar solvents. Taking into account these regularities, we decided to prepare new coumarin dyes with a quaternized pyridine ring which constitutes a part of a $\mathrm{BF}_{2}$ complex. For example, coumarin 312 contains a 2-(2-hydroxyphenyl)pyridine fragment as a chelating ligand. This compound is structurally similar to the strongly fluorescent $\mathrm{BF}_{2}$ complexes with the (2-quinolin-2-yl)phenol ligand. ${ }^{[139]}$ The fragment attached to $\mathrm{C}-3$ in compound 313 resembles the BOPIM complexes and has a 2(2-pyrrolyl)pyridine moiety which chelates the $\mathrm{BF}_{2}$ fragment. We also used the opportunity to link the nitrogen atoms of pyridine and pyrrole rings in the 2-(2-pyrrolyl)pyridine fragment via two methylene groups. This leads to a rigid heterocyclical system with the quaternized pyridine ring 314.

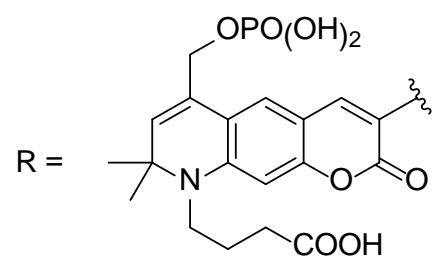
(1)

.



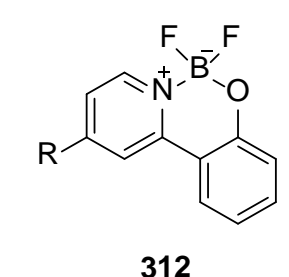

312

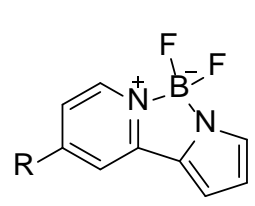

313<smiles></smiles>

314

Figure 45 Phosphorylated coumarins with a pyridinium group at C-3.

\subsubsection{Synthesis}

The preparation of the lipophilic dye $\mathbf{3 1 9}$ and an attempt to synthesize its phosphorylated analog $\mathbf{3 1 2}$ are given in Scheme 13. Analogously to 3-heteroarylcoumarins, building block 317 was prepared by esterification of salicylic aldehyde 289 with 4-(2bromopyridyl)acetic acid activated by DCC, and a subsequent Knoevenagel condensation. Similarly to compound 299, heteroaryl acetic acid 316 was prepared from 2-bromo- 
4-picoline 315. At this step, in order to evaluate spectral properties and stability of the corresponding coumarin- $\mathrm{BF}_{2}$ complex in polar protic solvents, we decided to prepare model compound 319 without the phosphate group. For this purpose, bromopyridylcoumarin 317 was subjected to a Suzuki reaction with 2-hydroxyphenylboronic acid to give coumarin 318 which contains the $\mathrm{N}, \mathrm{O}$-bidentate ligand. A chelation of $\mathrm{BF}_{3} \cdot \mathrm{Et}_{2} \mathrm{O}$ by compound 318 readily occurred in toluene in the presence of $\mathrm{NEt}_{3}$ and afforded coumarin- $\mathrm{BF}_{2}$ complex 319 in a good yield. When dissolved in $\mathrm{MeOH}$, this compound did not decompose upon prolonged standing, and therefore we decided to prepare the watersoluble analog 312. A detailed discussion and comparison of the spectral properties of coumarins 317-319 is given below in Section 2.2.3.

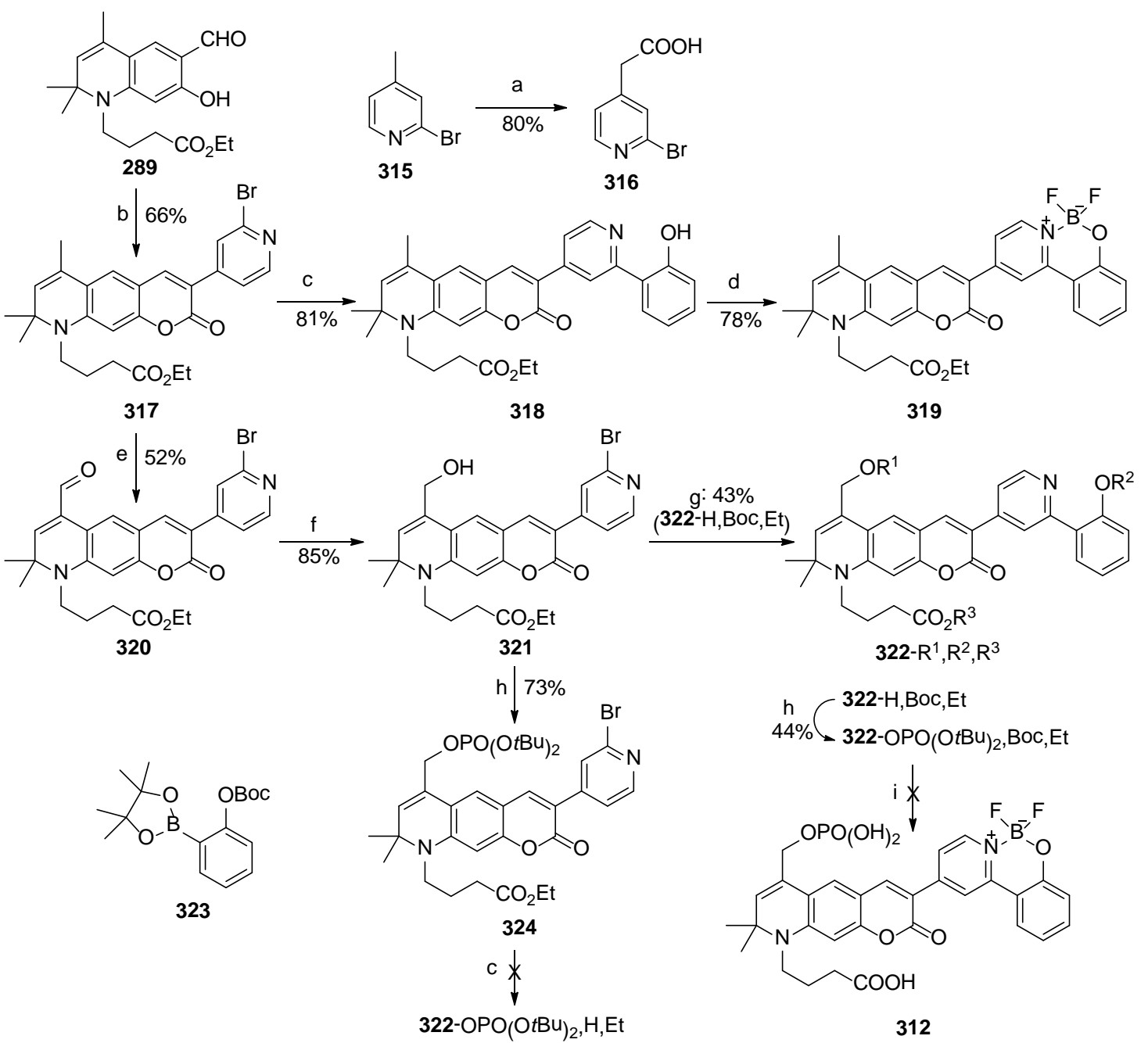

Scheme 13 Synthesis of phosphorylated coumarin 312: a) LDA, THF, $-78{ }^{\circ} \mathrm{C}, 1 \mathrm{~h}$; then $\mathrm{CO}_{2},-78^{\circ} \mathrm{C} \rightarrow$ r.t., overnight; b) 316, DCC, $\mathrm{NEt}_{3}$, DMAP, $\mathrm{CH}_{2} \mathrm{Cl}_{2} / \mathrm{DMF}, 30^{\circ} \mathrm{C}, 20 \mathrm{~h}$; c) 2-hydroxyphenylboronic acid, $\mathrm{Pd}\left(\mathrm{PPh}_{3}\right)_{4}$, toluene, aq. $\mathrm{Na}_{2} \mathrm{CO}_{3}, 120{ }^{\circ} \mathrm{C}$, overnight d) $\mathrm{BF}_{3} \cdot \mathrm{Et}_{2} \mathrm{O}$, $\mathrm{NEt}_{3}$, toluene, r.t., overnight; e) $\mathrm{SeO}_{2}$, dioxane, reflux, 3 h; f) $\mathrm{NaBH}_{4}, \mathrm{CeCl}_{3}$, THF/MeOH, $0{ }^{\circ} \mathrm{C}, 5$ min; g) 323, $\mathrm{Pd}\left(\mathrm{PPh}_{3}\right)_{4}$, toluene, aq. $\mathrm{Na}_{2} \mathrm{CO}_{3}$, 
$120{ }^{\circ} \mathrm{C}$, overnight; h) $i \mathrm{Pr}_{2} \mathrm{NP}(\mathrm{OtBu})_{2}, 1 \mathrm{H}$-tetrazole, $\mathrm{CH}_{2} \mathrm{Cl}_{2}, 40{ }^{\circ} \mathrm{C}, 1 \mathrm{~h}$; then $\mathrm{mCPBA}, 0{ }^{\circ} \mathrm{C}, 15 \mathrm{~min}$; i) aq. $\mathrm{NaOH}$, THF, water, r.t., overnight; then TFA, $\mathrm{CH}_{2} \mathrm{Cl}_{2}$, r.t., 1 h; then $\mathrm{BF}_{3} \cdot \mathrm{Et}_{2} \mathrm{O}, \mathrm{NEt}_{3}$, THF, r.t., 40 min.

For the synthesis of coumarin 312 - a phosphorylated analog of compound $\mathbf{3 1 9}$ - the allylic methyl group of bromopyridyl coumarin $\mathbf{3 1 7}$ was subjected to the same series of oxidation and reduction reactions as in the case of 3-heteroarylcoumarins in order to obtain alcohol 321 (Scheme 13). The phosphorylation according to the phosphoramidite method provided bromopyridylcoumarin 324 with a phosphate group. Unfortunately, attempted Suzuki reactions of $\mathbf{3 2 4}$ with 2-hydroxyphenylboronic acid failed to afford the desired compound 322-OPO(OtBu),H,Et, even though a number of various catalysts and ligands were tested. In contrast, a Suzuki reaction of coumarin 321 with O-tertbutoxycarbonyl-protected pinacol ester 323 proceeded well and gave compound 322H,Boc,Et in a moderate yield. The presence of a tert-butoxycarbonyl group, which masks the phenolic hydroxyl group, is important in the next step, in which a phosphate group is going to be attached to the allylic hydroxyl group using the protocol described above. The resulting compound 322-OPO $(\mathrm{OtBu})_{2}$,Boc,Et was subjected to a series of deprotection and complexation reactions in a one-pot fashion. First, the ethyl ester group was cleaved by basic hydrolysis. Then the tert-butyl groups on the phosphate function and the tertbutoxycarbonyl group attached to the aromatic hydroxyl were removed with TFA. Finally, upon exposure to $\mathrm{BF}_{3} \cdot \mathrm{Et}_{2} \mathrm{O}$ in the presence of $\mathrm{NEt}_{3}$, compound 312 was formed. However, due to instability on $\mathrm{SiO}_{2}$ or $\mathrm{RP}-\mathrm{SiO}_{2}$, all attempts to isolate compound 312 in a pure form failed. 


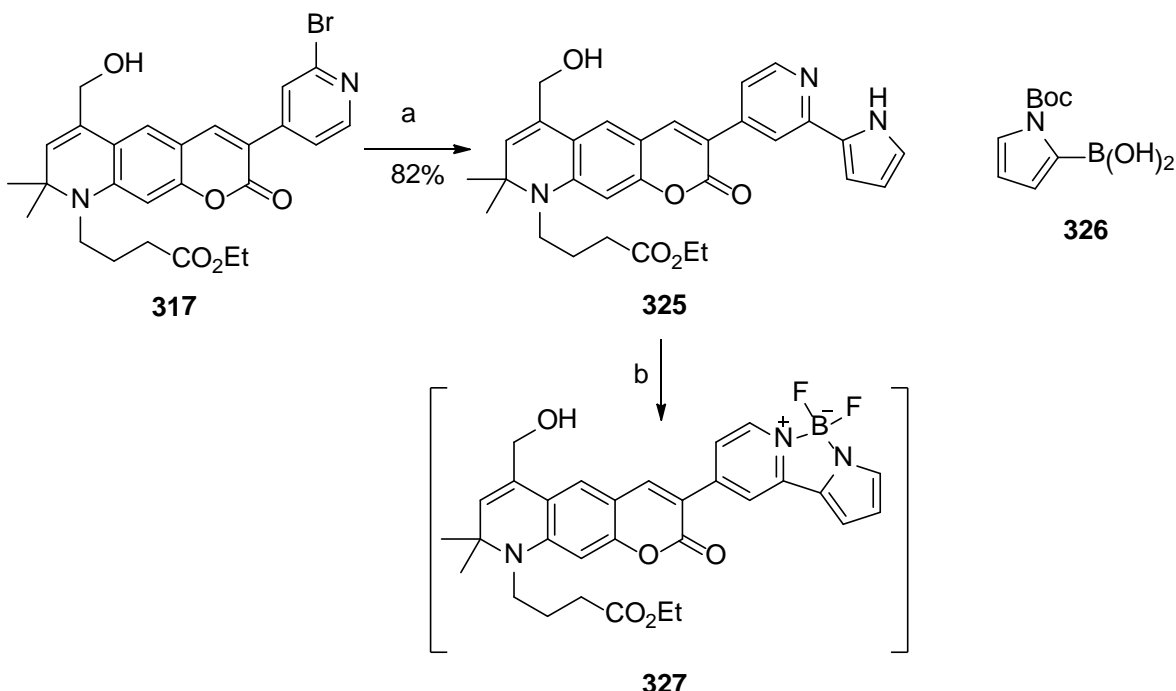

Scheme 14 Failed synthesis of coumarin- $\mathrm{BF}_{2}$ complex 327: a) 326, $\mathrm{Pd}\left(\mathrm{PPh}_{3}\right)_{4}$, toluene, aq. $\mathrm{Na}_{2} \mathrm{CO}_{3}, 120{ }^{\circ} \mathrm{C}$, overnight, then $150{ }^{\circ} \mathrm{C}, 30 \mathrm{~min}$; b) $\mathrm{BF}_{3} \cdot \mathrm{Et}_{2} \mathrm{O}, \mathrm{NEt}_{3}$, THF, r.t., overnight.

An attempted synthesis of the model coumarin 327 with a $\mathrm{BF}_{2}$ residue bound to the $N, N$ bidentate 2-(2-pyrrolyl)pyridine ligand is presented in Scheme 14. The pyrrolyl residue was introduced to bromopyridylcoumarin $\mathbf{3 1 7}$ by a Suzuki reaction with the commercially available $N$-tert-butoxycarbonyl protected 2-pyrrolylboronic acid 326. The pyrolysis of the reaction mixture at $150{ }^{\circ} \mathrm{C}$ cleaved the tert-butoxycarbonyl-protecting group and provided coumarin 325 in good yield. The reaction of 325 with $\mathrm{BF}_{3} \cdot \mathrm{Et}_{2} \mathrm{O}$ in THF in the presence of $\mathrm{NEt}_{3}$ yielded a red fluorescent solution. However, upon quenching the reaction mixture with $\mathrm{MeOH}$ or $\mathrm{H}_{2} \mathrm{O}$, the fluorescent product (complex 327) decomposed and left the yellow-colored starting material 325. Thus, coumarin 327 turned out to be even less stable than coumarin $\mathbf{3 1 2}$.

Then we studied the feasibility of the preparation of the lipophilic analog of the dye $\mathbf{3 1 4}$. The straightforward synthetic route involves a reaction of compound 325 with dibromoethane (Scheme 15). To our sursprise, the only fluorescent product observed in this reaction mixture was the dehydrogenated compound 328. However, the preparative yield was very low, even when prolonged reaction times, elevated temperatures and saturation of the reaction mixture with air were applied. Due to the interesting and promising spectral properties of compound $\mathbf{3 2 8}$ (see Section 2.2.3 for further details), we decided to develop a more efficient synthetic route. For that, we tried to condense compound 325 with chloroacetaldehyde diethyl acetal. Under the known conditions, ${ }^{[140]}$ the reaction did not proceed at all after heating for $2 \mathrm{~h}$ at $100{ }^{\circ} \mathrm{C}$. However, when an excess of this acetal and 
a stoichiometric amount of $\mathrm{NaI}$ were added, the starting material 325 was completely consumed after 2 days at $100{ }^{\circ} \mathrm{C}$. Notably, the product of this transformation - compound 329 - did not readily undergo dehydration to $\mathbf{3 3 0}$ when exposed to acid. This behavior may be attributed to the inability of this rigid polycyclic system to adopt the antiperiplanar conformation in the transition state of the elimination reaction. The phosphorylation reaction transformed both hydroxyl groups present in compound 329 into the corresponding phosphates. As a result, the hydroxyl belonging to the hemiaminal fragment was converted to a good leaving group and readily eliminated during the isolation procedure. Thus, coumarin 330 with the pyrido[1,2-a]pyrrolo[2,1-c]pyrazinium moiety at C-3 was obtained. However, the final deprotection step failed to give compound $\mathbf{3 3 1}$. Probably, the attack of the hydroxide ion on the double bond prone to undergo nucleophilic addition triggered the decomposition reaction.



Scheme 15 Synthesis of coumarins with a pyrido[1,2-a]pyrrolo[2,1-c]pyrazinium substituent: a) $\mathrm{BrCH}_{2} \mathrm{CH}_{2} \mathrm{Br}, 130^{\circ} \mathrm{C}$, 3 days; b) $\mathrm{ClCH}_{2} \mathrm{CH}(\mathrm{OEt})_{2}$, NaI, DMF, $100^{\circ} \mathrm{C}, 5 \mathrm{~h}$; c) $i \operatorname{Pr}_{2} \mathrm{NP}(\mathrm{OtBu})_{2}, 1 H$-tetrazole, $\mathrm{CH}_{2} \mathrm{Cl}_{2}, 40{ }^{\circ} \mathrm{C}, 1 \mathrm{~h}$; then $\mathrm{mCPBA}, 0{ }^{\circ} \mathrm{C}, 15 \mathrm{~min}$; d) aq. $\mathrm{NaOH}$, THF, water, r.t., overnight; then TFA, $\mathrm{CH}_{2} \mathrm{Cl}_{2}$, r.t., 1 h.

\subsubsection{Spectral properties}

Spectral properties of synthesized compounds 317-319, 325, 328 were measured in $\mathrm{MeOH}$ and are presented in Table 10. Introduction of the bromine atom to the pyridine ring in coumarin 317 caused bathochromic and bathofluoric shifts of 14 and $4 \mathrm{~nm}$, respectively, in comparison with the parent coumarin 282. Interestingly, the presence of the 
bromine atom, which usually quenches the emission by increasing the rate of intersystem crossing, ${ }^{[141]}$ did not have any effect on the fluorescence QY of $\mathbf{3 1 7 .}$

2-(2'-Hydroxyphenyl)pyridine is known to undergo an ESIPT process upon excitation. ${ }^{[142]}$ In the case of coumarin 318, which has the 2-(2'-hydroxyphenyl)pyridyl moiety, ESIPT may also be responsible for the low fluorescence QY in $\mathrm{MeOH}$. Upon excitation, the excited state $S_{1}$ of the "normal" (enol)-tautomer is formed initially. Afterwards, due to the increased basicity of the pyridinic nitrogen and acidity of the phenolic hydroxyl, a very fast proton transfer takes place within the molecule resulting in the formation of the non-emissive keto-tautomer 318K (see Figure 46) in the excited state $\mathrm{S}_{1}$ '. A small fraction of molecules that did not undergo ESIPT gives rise to a very weak fluorescence with a "normal” (typical for coumarin dyes) Stokes shift of $66 \mathrm{~nm}$.

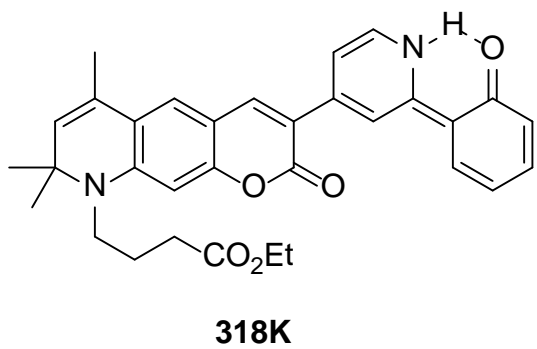

Figure 46 Keto-tautomer $\mathbf{3 1 8 K}$ of coumarin $\mathbf{3 1 8}$ formed due to ESIPT from the phenolic hydroxyl to the pyridine nitrogen.

Coordination of the $\mathrm{BF}_{2}$ moiety with 2-(2-hydroxyphenyl)pyridyl fragment in coumarin 318 completely suppresses the ESIPT. As a result, emission efficiency of the complex 319 significantly increases in comparison to the parent compound 318. Moreover, the partial positive charge on the pyridinic nitrogen facilitates the ICT in coumarin 319 and leads to large bathochromic and bathofluoric shifts (26 and $53 \mathrm{~nm}$ relative to compound 318).

The unfavorable geometry and the lower acidity of the pyrrole ring in the 2-(2'-pyrrolyl)pyridyl fragment of compound 325 apparently makes the ESIPT impossible. As a consequence, this compound shows only one emission band with a maximum at $505 \mathrm{~nm}$ with a "normal" Stokes shift of $73 \mathrm{~nm}$ and a good fluorescence QY in MeOH. The presence of the pyrrole ring does not influence the positions of absorption and fluorescence maxima, when compared to 3-(2-pyridyl)coumarin 282. 
Among all compounds presented in this section, coumarin 328 with the polycyclic and rigid pyrido[1,2-a]pyrrolo[2,1-c]pyrazinium substituent displays the most red-shifted absorption and emission maxima. In addition, this compound features a large Stokes shift of $98 \mathrm{~nm}$ and a high value of the fluorescence QY in $\mathrm{MeOH}$. These attractive spectral properties make this fluorophore particularly interesting for further developments (e.g. decoration with polar groups and amino-reactive residues).

Table 10 Spectral properties of 3-pyridiniumcoumarins and their precursors in $\mathrm{MeOH}$.

\begin{tabular}{cccccc}
\hline Compound & $\lambda_{\text {abs,max }}, \mathrm{nm}$ & $\varepsilon, \mathrm{M}^{-1} \cdot \mathrm{cm}^{-1}$ & $\lambda_{\mathrm{em}, \max }, \mathrm{nm}$ & $\Delta \lambda, \mathrm{nm}$ & $\Phi_{\mathrm{fl}}$ \\
\hline $\mathbf{3 1 7}$ & 445 & 38400 & 515 & 70 & 0.72 \\
$\mathbf{3 1 8}$ & 440 & 40000 & 506 & 66 & 0.005 \\
$\mathbf{3 1 9}$ & 466 & 32400 & 559 & 93 & 0.38 \\
$\mathbf{3 2 5}$ & 432 & 33650 & 505 & 73 & 0.50 \\
$\mathbf{3 2 8}$ & 489 & $\mathrm{n} / \mathrm{d}$ & 587 & 98 & 0.56 \\
\hline
\end{tabular}

\subsubsection{Conclusion and outlook}

The new coumarins 319 and $\mathbf{3 2 8}$ with a positively charged 4-pyridinium fragment attached to C-3 have been prepared from the universal precursor 289. Both compounds exhibited absorption maxima beyond $450 \mathrm{~nm}$ and emitted light in the green-yellow spectral region with large Stokes shifts. Phoshorylated analog of 319 apparently had a poor hydrolytic stability, and could not be isolated in pure form. Absorption and emission maxima of coumarin 328 are close to those of commercial dyes Chromeo 494, DY480XL, DY-485XL, DyLight 485-LS and Abberior Star 470SXP. Therefore, it is particularly interesting to develop a synthetic approach to a water-soluble analog of $\mathbf{3 2 8}$ and evaluate its performance in optical microscopy in comparison with commercially available dyes. 


\subsection{Synthesis of pyrido- and isoquinolino-fused coumarin dyes}

\subsubsection{Motivation and key structural elements}

Coumarin dyes are known to have moderate photostabilities in aqueous media. For example, the average numbers of the excitation cycles before bleaching $(\mu)$ for coumarins was found to be approximately 2 to 3 orders of magnitude smaller than the corresponding values for rhodamine dyes. ${ }^{[143]}$ This poorer photostability can be explained by a generally higher chemical reactivity of the coumarin fluorophore towards Michael addition of nucleophiles and a larger triplet quantum yield in comparison with rhodamines. Due to its comparatively long lifetime, the formation of the lowest triplet state is believed to be the main bleaching pathway. Moreover, the high irradiation intensities applied in confocal and super-resolution (STED) microscopies, can give rise to multiphoton absorption. As a result, various photochemical reactions from the higher excited states $S_{n}$ may form additional photobleaching channels which cause further deterioration of the fluorophore in the sample of interest.

For coumarins without substituents at C-3 and C-4, a photodimerization reaction may represent a prevailing photodegradation mechanism. ${ }^{[144]}$ In the case of $7-N, N$-diethylamino-4-methylcoumarin two main photobleaching paths were identified: (1) dealkylation of the 7-diethylamino group with formation of 7-monoethylamino- and 7-aminocoumarins and (2) oxidation of the methyl group at C-4 to carboxylic acid via intermediate formation of 4-hydroxymethylene- and 4-formyl derivatives. ${ }^{[145]}$ The formation of oxidation products during irradiation often involves "self-sensitized" oxidation processes. According to this mechanism, a transfer of the excitation energy between a dye molecule in the triplet state $\mathrm{T}_{1}$ and triplet oxygen occurs initially. Afterwards, the resulting singlet oxygen oxidizes the dye in the ground state $S_{0}{ }^{[146]}$ 


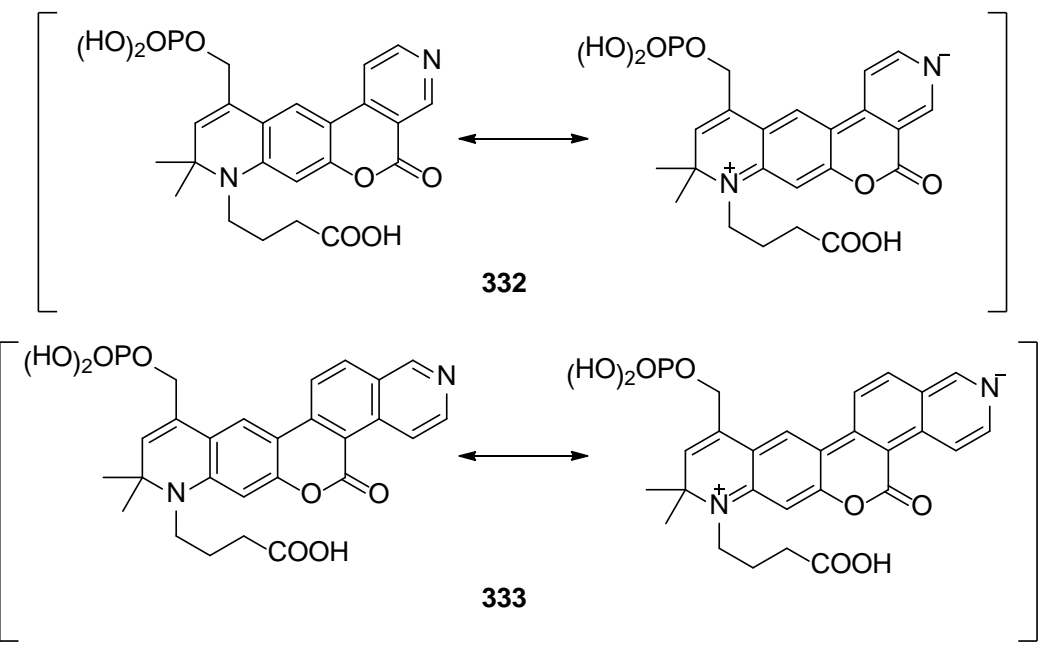

Figure 47 Phosphorylated pyrido- and isoquinolino-fused coumarins $\mathbf{3 3 2}$ and $\mathbf{3 3 3}$ and corresponding mesomeric structures showing the participation of the pyridin and isoquinoline moieties in delocalization of the lone electron pair on the nitrogen atom of the 7-amino group.

Benzo[c]coumarins do not have a reactive double bond conjugated with the carbonyl group of coumarin and, therefore, cannot undergo photodimerization. Fusion with an additional benzene ring results in bathochromic and bathofluoric shifts in comparison with the corresponding 3,4-unsubstituted analogs. ${ }^{[147]}$ Taking into account these attractive features of benzo[c]-fused coumarins, we decided to prepare pyrido[3,4-c]-fused coumarin 332 and isoquinolino[3,4-f]-fused coumarin 333 (Figure 47). Similarly to the fused benzene fragment in benzo[c]coumarins, pyridine and isoquinoline moieties in dyes $\mathbf{3 3 2}$ and 333 provide planar and rigid molecular frameworks and protect "critical” positions susceptible to (photo)oxidation and photodimerization. Furthermore, due to the negative mesomeric effect, they could participate in delocalization of the lone electron pair of the amino group at C-7 to the pyridine or isoquinoline nitrogen. This delocalization facilitates ICT in the excited state and provides large shifts of absorption and emission spectra towards the red region of the visible spectrum.

\subsubsection{Synthesis of model hydrophilic compounds}

A route to the model compound $343-\mathrm{OH}$ without the phosphate group is given in Scheme 16. Similarly to the syntheses of 3-substituted coumarins described in Sections 2.1 and 2.2, we started to assemble the pyrido- and isoquinolino-fused coumarins with the preparation of the universal precursor 336. The latter was prepared in two steps with a high overall yield starting from the commercially available 4-bromo-3-methoxyaniline 
334. The chemical transformations described earlier (see Section 2.1.2) afforded compound 336. It represents an aryl bromide which can be used in cross-coupling reactions with an organometallic derivative of the nicotinic acid with a leaving group at C-4 (as in compound 341). After unmasking the phenolic group at C-7, the resulting intermediate is expected to form the desired pyridocoumarin. However, the required organometallic compounds, which can serve as coupling partners for the synthesis of pyridocoumarins, are predominantly unknown, probably due to their instability (we found only one example $\left.{ }^{[148]}\right)$. All attempts to prepare the trimethylstannyl-substituted nicotinic acid ester 341 failed. Therefore, we decided to synthesize an organometallic derivative from the precursor 336 and couple it with halonicotinic acid derivatives, many of which are commercially available. To our regret, all Pd-catalyzed borylation and stannylation reactions yielded only the product of debromination 337 .

The traditional method for the preparation of boronic esters involves the trapping of an aryllithium reagent with a borate ester. In this respect, compound $\mathbf{3 3 6}$ is incompatible with this method due to the presence of the ester group. Therefore, we reduced ester 336 with $\mathrm{LiAlH}_{4}$ and obtained alcohol 339-H in an excellent yield. Later, in the final steps of the synthesis, the hydroxyl group could be easily converted to the amino-reactive $\mathrm{N}$ hydroxysuccinimidyl carbonate and used for bioconjugation. For the reaction with $t \mathrm{BuLi}$, the hydroxyl group in compound 339-H was protected and used as a tetrahydropyranyl derivative. The intermediate bromide 339-THP was subjected to lithium-bromine exchange, and the organolithium compound was then quenched with triisopropyl borate. Direct transesterification in the reaction mixture using pinacol in the presence of acetic acid afforded boronate ester 338. The folllowing Suzuki cross-coupling between $\mathbf{3 3 8}$ and commercially available 2-chloro-4-iodonicotinic acid proceeded smoothly and gave the biaryl 342-THP in good yield. The methoxy and tetrahydropyranyl groups in compound 342-THP, in principle, could be removed in one synthetic step using two equivalents of $\mathrm{BBr}_{3}$. However, initial experiments showed that even a large excess of $\mathrm{BBr}_{3}$ cleaved only the tetrahydropyranyl group and left the methoxy group intact. To overcome this problem, we replaced the THP group with the acetate protective group using the known one-pot procedure. ${ }^{[149]}$ After that, acetate 342 -Ac was subjected to a reaction with $\mathrm{BBr}_{3}$. Unfortunately, the demethylation turned out to be sluggish, and a large excess of $\mathrm{BBr}_{3}$ was required to provide full consumption of the starting material 342-Ac in an acceptable time. 




Scheme 16 Synthesis of the model coumarins 343-OH and 344: a) acetone, $\mathrm{Yb}(\mathrm{OTf})_{3}$, r.t., 16 h; b) ethyl 4iodobutanoate, DIEA, $105{ }^{\circ} \mathrm{C}, 64 \mathrm{~h}$; c) hexabutylditin, $\mathrm{Pd}\left(\mathrm{PPh}_{3}\right) \mathrm{Cl}_{2}$, toluene, $110{ }^{\circ} \mathrm{C}, 3$ days; d) bis(pinacolato)diboron, $\mathrm{PdCl}_{2}$ (dppf), AcOK, DMSO, $80^{\circ} \mathrm{C}$, overnight; e) $t \mathrm{BuLi}$, THF, $-78^{\circ} \mathrm{C}, 45 \mathrm{~min}$; then $\mathrm{B}(\mathrm{OiPr})_{3},-78^{\circ} \mathrm{C} \rightarrow$ r.t., overnight; then $\mathrm{AcOH}$, pinacol, r.t., $1 \mathrm{~h}$; f) $\mathrm{LiAlH}_{4}, \mathrm{THF}, 0{ }^{\circ} \mathrm{C} \rightarrow$ r.t.; 2.5 h; g) $2-$ chloro-4-iodonicotinic acid, $\mathrm{Pd}\left(\mathrm{PPh}_{3}\right)_{4}$, aq. $\mathrm{Na}_{2} \mathrm{CO}_{3}$, toluene/EtOH, reflux, $18 \mathrm{~h}$; h) 3,4-dihydro- $2 \mathrm{H}$-pyrane, $\mathrm{TsOH} \cdot \mathrm{H}_{2} \mathrm{O}, \mathrm{CH}_{2} \mathrm{Cl}_{2} ;{ }^{\circ} \mathrm{C} \rightarrow$ r.t., overnight; i) hexamethylditin, $\mathrm{Pd}\left(\mathrm{PPh}_{3}\right) \mathrm{Cl}_{2}$, dioxane, $100{ }^{\circ} \mathrm{C}$, overnight; j) $\mathrm{BBr}_{3}, \mathrm{CH}_{2} \mathrm{Cl}_{2}, 0{ }^{\circ} \mathrm{C} \rightarrow$ r.t., overnight; $\mathrm{k}$ ) $\mathrm{MeI}, \mathrm{MeCN}, 75^{\circ} \mathrm{C}, 2$ days; l) $\mathrm{AcOH}, \mathrm{AcCl}$, r.t., 1 h.

As expected, in the course of this process a spontaneous ring closure occurred, and the pyridocoumarin core skeleton was formed. The harsh reaction conditions caused partial cleavage of the acetate protective group. As a result, three main products were obtained in this reaction: acetate $343-\mathrm{OAc}$, alcohol $343-\mathrm{OH}$ and bromide $343-\mathrm{Br}$. In the experiments aimed at the quaternization of the pyridine nitrogen in compound 343-OAc we isolated methylpyridonocoumarin 344 which apparently resulted from hydrolysis of the $\mathrm{N}$ methylated 2-chlropyridine 345 during chromatographic isolation on $\mathrm{SiO}_{2}$ (Scheme 17). 
<smiles></smiles>

345<smiles>CC(=O)OCCCCN1c2cc3oc(=O)c4c(=O)n(C)ccc4c3cc2C(C)=CC1(C)O</smiles>

344

Scheme 17 Decomposition of compound 345 on silica during the isolation procedure.

Searching for faster and more efficient synthetic routes to pyridocoumarins, we tried to condense formamide with 3-formyl-4-methylcoumarin 348 which, in turn, was prepared from commercially available compound 346 in two steps. We found that condensation of 348 with formamide occurs in the presence of conc. $\mathrm{H}_{2} \mathrm{SO}_{4}$ at elevated temperatures (Scheme 18). Under these conditions, the full conversion of $\mathbf{3 4 8}$ was not reached due to decomposition of formamide, and target compound $\mathbf{3 4 9}$ was isolated in a low yield of $27 \%$.<smiles>Cc1cc(=O)oc2cc3c(cc12)CCCN3</smiles>

346

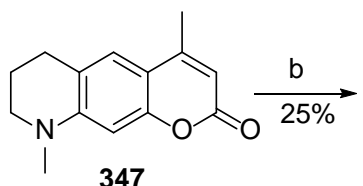

347

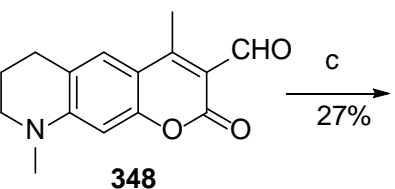

348

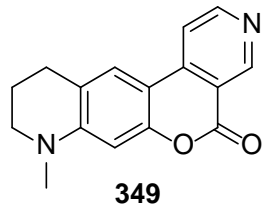

349

Scheme 18 Synthesis of model coumarin 349: a) trimethyl phosphate, $195{ }^{\circ} \mathrm{C}$, overnight; b) $\mathrm{POCl}_{3}, \mathrm{DMF}$, $50{ }^{\circ} \mathrm{C}, 10 \mathrm{~min}$; c) formamide, $\mathrm{H}_{2} \mathrm{SO}_{4}, 90^{\circ} \mathrm{C}$, overnight.

For the synthesis of the model isoquinolinocoumarin 354 we used another synthetic approach which is, however, analogous to the one depicted in Scheme 16. The crosscoupling partner for the boronate ester $\mathbf{3 3 8}$ was prepared from the commercially available 6-bromoisoquinoline $\mathbf{3 0 0}$ in three synthetic steps (Scheme 19). First, the bromoisoquinoline 300 was nitrated with a mixture of conc. $\mathrm{H}_{2} \mathrm{SO}_{4}$ and $\mathrm{HNO}_{3}$. The regioselectivity of this reaction was good, and the expected isomer $\mathbf{3 5 0}$ was isolated in excellent yield. In the next step, the nitro compound 350 was reduced to the corresponding aromatic amine 351 using iron in the presence of $\mathrm{NH}_{4} \mathrm{Cl}$, which acted as a weak acid. The method of generation of the diazonium cation from compound 351 plays an important role in the following reaction with $\mathrm{CuCN}$. Thus, when 351 was treated with aq. $\mathrm{HNO}_{2}$ (prepared in situ from $\mathrm{NaNO}_{2}$ and $\mathrm{H}_{2} \mathrm{SO}_{4}$ ), only phenol $352-\mathrm{OH}$ was isolated as the main product. 
Under water-free conditions, when the corresponding diazonium cation was generated by action of $t$-butyl nitrite on compound 351 in DMSO ${ }^{[150]}$ the target nitrile 352-CN was obtained, although in a low yield.
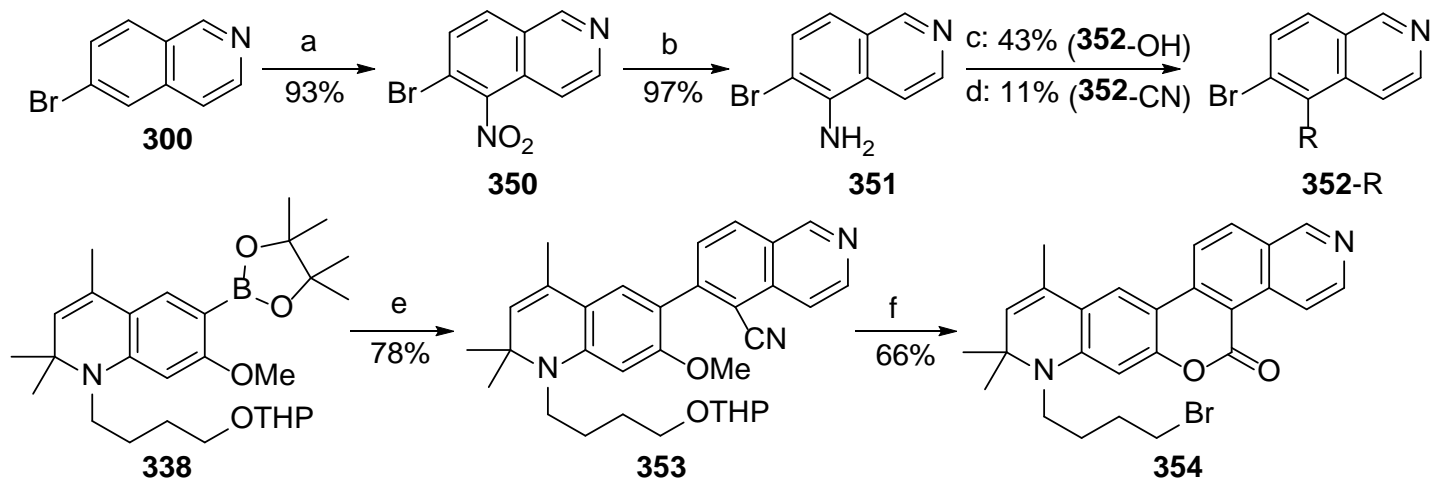

350

351

352-R

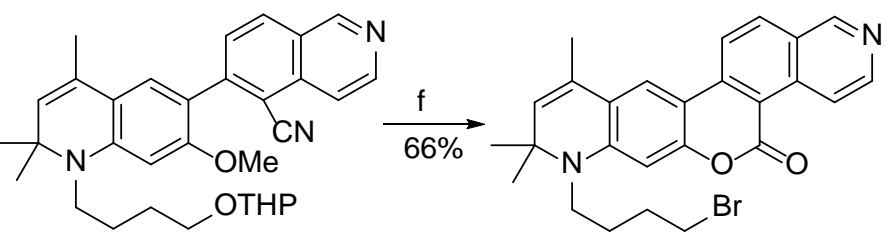

353

354

Scheme 19 Synthesis of the model coumarin 354: a) $\mathrm{HNO}_{3}, \mathrm{H}_{2} \mathrm{SO}_{4}$, r.t., 2 h; b) iron powder, $\mathrm{NH}_{4} \mathrm{Cl}$, EtOH/ $\mathrm{H}_{2} \mathrm{O}$, r.t., 50 min; c) $\mathrm{NaNO}_{2}, \mathrm{H}_{2} \mathrm{SO}_{4}, \mathrm{CuCN}, \mathrm{NaCN}, \mathrm{H}_{2} \mathrm{O}$, toluene, $0{ }^{\circ} \mathrm{C}, 30 \mathrm{~min}$; then $70{ }^{\circ} \mathrm{C}, 2.5 \mathrm{~h}$; d) $t$ BuONO, CuCN, DMSO, $40{ }^{\circ} \mathrm{C}, 1.5 \mathrm{~h}$; e) 352-CN, $\mathrm{Pd}\left(\mathrm{PPh}_{3}\right)_{4}$, aq. $\mathrm{Na}_{2} \mathrm{CO}_{3}$, toluene, $110{ }^{\circ} \mathrm{C}$, overnight; f) conc. aq. $\mathrm{HBr}, 130^{\circ} \mathrm{C}, 3 \mathrm{~h}$.

A cross-coupling reaction of compound 352-CN with boronate ester 338 gave the biaryl 353. The final deprotection followed by the intramolecular cyclization in refluxing aqueous $\mathrm{HBr}$ afforded isoquinolinocoumarin 354 in a good yield. Upon standing, solutions of compound 354 spontaneously formed a red colored substance which may result from an alkylation of the pyridine nitrogen with the alkyl bromide part of another molecule of compound 354.

In another synthetic approach leading to (iso)quinolinocoumarins, the key step is a crosscoupling reaction between organoboron compounds of the type 357 or organotin compounds of the type 358 and halopyridinecarboxaldehydes with the vicinal halogen and formyl groups (Scheme 20). The subsequent intramolecular condensation of the formyl group, which belongs to the pyridine fragment, with the methyl group at C-4 of the coumarin leads to an additional benzene ring, thus resulting in the (iso)quinolinocoumarin fluorophore.

The preparation of organometallic compounds 357 and 358 was started with the bromination of the commercially available 7- $N, N$-dimethylamino-4-methylcoumarin 355-H with bromine in $\mathrm{AcOH}$. Then bromide 355- $\mathrm{Br}$ was subjected to Pd-catalyzed borylation and stannylation reactions to afford boronate ester 357 and 3-(trimethylstannyl)coumarin 358, 
respectively. Along with the desired product 357, the borylation reaction produced substantial amounts of the debrominated compound $355-\mathrm{H}$ which, due to its similar $R_{\mathrm{f}}$, significantly complicated the chromatographic isolation of 357. The stannylation of 355Br with hexamethylditin was carried out according to the known protocol for the closely related $2 H$-pyran-2-ones, ${ }^{[151]}$ and proceeded cleanly, though the preparative yield of compound 358 was moderate. When the less toxic hexabutylditin was used, the stannylation did not occur at all.



Scheme 20 Synthesis of model coumarins 356, 360 and 362: a) $\mathrm{Br}_{2}$, AcOH, r.t., 15 min; b) bis(pinacolato)diboron, $\mathrm{PdCl}_{2}(\mathrm{dppf})$, AcOK, dioxane, $80{ }^{\circ} \mathrm{C}$; c) $\mathrm{Sn}_{2} \mathrm{Me}_{6}, \mathrm{Pd}\left(\mathrm{PPh}_{3}\right)_{4}$, toluene, $110{ }^{\circ} \mathrm{C}$, overnight; d) 3-bromopyridine-2-carbaldehyde, $\mathrm{CuCl}$, $\mathrm{LiCl}, \mathrm{Pd}\left(\mathrm{PPh}_{3}\right)_{4}$, $\mathrm{DMSO}, 60{ }^{\circ} \mathrm{C}$, overnight; e) 2-chloro-4iodopyridine-3-carbaldehyde, $\mathrm{CuCl}, \mathrm{LiCl}, \mathrm{Pd}\left(\mathrm{PPh}_{3}\right)_{4}$, DMSO, $60{ }^{\circ} \mathrm{C}$, overnight; f) 2-bromopyridine-3carbaldehyde, $\mathrm{Pd}\left(\mathrm{PPh}_{3}\right)_{4}$, aq. $\mathrm{Na}_{2} \mathrm{CO}_{3}$, toluene, $110{ }^{\circ} \mathrm{C}$, overnight; g) 2-bromopyridine-3-carbaldehyde, $\mathrm{CuCl}$, LiCl, $\mathrm{Pd}\left(\mathrm{PPh}_{3}\right)_{4}$, DMSO, $60{ }^{\circ} \mathrm{C}$, overnight; h) MeI, DMF, $100{ }^{\circ} \mathrm{C}$, overnight; i) $\mathrm{Cs}_{2} \mathrm{CO}_{3}$, EtOH, r.t., 3 h.

In the course of a Suzuki reaction of the boronate ester 357 with 2-bromopyridine-3carbaldehyde, a spontaneous intramolecular cyclization with the formation of quinolinocoumarin 360 took place. In a related Stille coupling of the organotin compound $\mathbf{3 5 8}$ with 2-bromopyridine-3-carbaldehyde, in the absence of a strong base, it was possible to isolate the intermediate biaryl 361. The Corey modification of the Stille coupling procedure $^{[152]}$ provided an excellent yield of $\mathbf{3 6 1}$. The intramolecular cyclization of coumarin 361 readily occurred in EtOH in the presence of catalytic amounts of $\mathrm{Cs}_{2} \mathrm{CO}_{3}$ and afforded quinolinocoumarin 360. Surprisingly, in the Stille reactions of compound 358 with 3-bromopyridine-2-carbaldehyde and 2-chloro-4-iodopyridine-3-carbaldehyde only 
the cyclized products 356 and 359, respectively, were isolated. In an attempt to convert the chloropyridine moiety in compound 359 into an $N$-methylpyridone fragment (as in compound 344), the quaternized isoquinolinocoumarin 362 was obtained as a main product.

The structure of quinolinocoumarin 373 (Scheme 21) is closer to the target coumarin 333, than, for example, the structure of coumarin 360. In the synthesis of model compound 373, phenol 288- $\mathrm{H}$ was subjected to a Pechmann condensation with ethyl acetoacetate and gave coumarin 362. $\mathrm{SeO}_{2}$ in dioxane selectively oxidized the methyl group at the $\mathrm{C}=\mathrm{C}$-bond in dihydroquinoline fragment of coumarin 362, and aldehyde 363 was formed. The subsequent bromination of $\mathbf{3 6 3}$ with bromine in $\mathrm{AcOH}$ gave compound $\mathbf{3 6 4}$. The organotin derivative 368 was obtained from 364 by a Pd-catalysed stannylation with hexamethylditin. In the later steps, the synthetic strategy was essentially the same as for compound 360. Thus, a Stille coupling reaction of $\mathbf{3 6 8}$ with 2-bromopyridine-2-carbaldehyde 365 led to the formation of biaryl 371 as an intermediate, which cyclized to quinolinocoumarin 370 in the presence of $\mathrm{Cs}_{2} \mathrm{CO}_{3}$ in EtOH. The reduction of $\mathbf{3 7 0}$ with $\mathrm{NaBH}_{4}$ was found to give many products. As a result, the preparative yield of coumarin 373 was very low. One of the main side-products of this reduction is an unindentified overreduced unpolar substance which was readily oxidized to compound $\mathbf{3 7 3}$ on a TLC plate or in solution while standing in air. The re-oxidation of the reaction mixture with $\mathrm{Bu}_{4} \mathrm{NIO}_{4}$ directly after full consumption of compound $\mathbf{3 7 0}$ helped to provide an acceptable preparative yield of quinolinocoumarin 373. In contrast to quinolinocoumarin 370, 3-heteroarylated coumarins could be reduced in excellent yields. Therefore, we decided to perform the ring closure reaction after the reduction step in order not to expose the sensitive heterocyclic system to strong reducing agents. However, bromide $\mathbf{3 6 7}$ with the reduced aldehyde group did not react with hexamethylditin. This result indicates that the reduction step should be undertaken only after stannylation.

In new synthetic approach, 2-bromopyridine-3-carbaldehyde 365 was protected as a dioxolane moiety by reaction with ethylene glycol. The product of this reaction - compound 369 - was introduced into a Stille reaction with organotin compound 368 and afforded the biaryl 372. As expected, reduction of $\mathbf{3 7 2}$ proceeded cleanly and gave the coumarin 375 in a high yield. The subsequent deprotection with $\mathrm{TsOH}$ in aqueous acetone afforded compound $\mathbf{3 7 4}$ with a free aldehyde group. Treatment of 374 with $\mathrm{Cs}_{2} \mathrm{CO}_{3}$ in $\mathrm{EtOH}$ induced an intramolecular condensation and yielded the target quinolinocoumarin 
373. Despite two additional steps, the overall yield in the synthetic sequence $\mathbf{3 6 8} \rightarrow \mathbf{3 7 2}$ $\rightarrow 375 \rightarrow 374 \rightarrow 373$ (38\%) is almost twice as high as the overall yield achieved in the shorter sequence $\mathbf{3 6 8} \rightarrow \mathbf{3 7 1} \rightarrow \mathbf{3 7 0} \rightarrow \mathbf{3 7 3}$ (20\%).

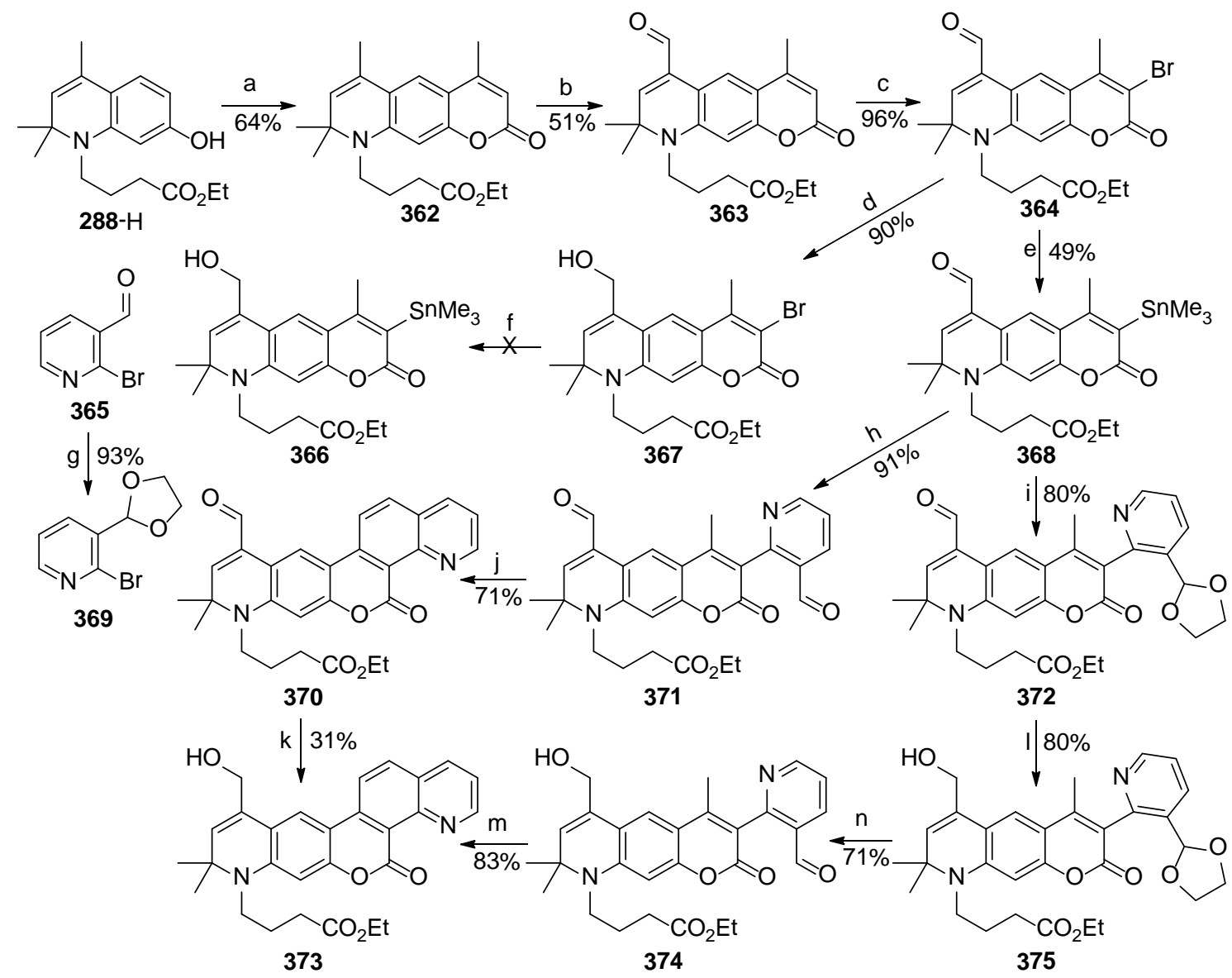

Scheme 21 Synthesis of model coumarin 373: a) ethyl acetoacetate, $\mathrm{ZnCl}_{2}$, EtOH. $90{ }^{\circ} \mathrm{C}, 20 \mathrm{~h}$; b) $\mathrm{SeO}_{2}$, dioxane, $100{ }^{\circ} \mathrm{C}, 3 \mathrm{~h}$; c) $\mathrm{Br}_{2}$, $\mathrm{AcOH}$, r.t., 10 min; d) $\mathrm{NaBH}_{4}, \mathrm{CeCl}_{3}$, THF, $\mathrm{MeOH}, 0^{\circ} \mathrm{C}, 10 \mathrm{~min}$; e) $\mathrm{Sn}_{2} \mathrm{Me}_{6}$, $\mathrm{Pd}\left(\mathrm{PPh}_{3}\right)_{4}$, toluene, $110{ }^{\circ} \mathrm{C}, 25 \mathrm{~h}$; f) $\mathrm{Sn}_{2} \mathrm{Me}_{6}, \mathrm{Pd}\left(\mathrm{PPh}_{3}\right)_{4}$, toluene, $110{ }^{\circ} \mathrm{C}$, overnight; g) ethylene glycol, $\mathrm{TsOH} \cdot \mathrm{H}_{2} \mathrm{O}$, toluene, reflux, 5 h; h) 2-bromopyridine-3-carbaldehyde, $\mathrm{CuCl}, \mathrm{LiCl}, \mathrm{Pd}\left(\mathrm{PPh}_{3}\right)_{4}, \mathrm{DMSO}, 60$ ${ }^{\circ} \mathrm{C}$, overnight; i) 369, $\mathrm{CuCl}, \mathrm{LiCl}, \mathrm{Pd}\left(\mathrm{PPh}_{3}\right)_{4}$, DMSO, $60{ }^{\circ} \mathrm{C}$, overnight; k) $\mathrm{NaBH}_{4}$, EtOH, THF, r.t., 5 min; then aq. $\mathrm{HClO}_{4}, \mathrm{Bu}_{4} \mathrm{NIO}_{4}$, r.t., 5 min; l) $\mathrm{NaBH}_{4}$, $\mathrm{MeOH}$, THF, $0{ }^{\circ} \mathrm{C}, 5$ min; m) $\mathrm{Cs}_{2} \mathrm{CO}_{3}$, EtOH, r.t., 1.5 h; n) acetone, $\mathrm{H}_{2} \mathrm{O}, \mathrm{TsOH} \cdot \mathrm{H}_{2} \mathrm{O}$, reflux, overnight.

\subsubsection{Spectral properties of pyrido- and (iso)quinolinocoumarins}

Spectral properties of pyrido- and (iso)quinolinocoumarins are presented in Table 11. When dissolved in $\mathrm{CH}_{2} \mathrm{Cl}_{2}$ or $\mathrm{MeOH}$, pyridocoumarin, 343-OAc absorbs in the violet region of the visible spectrum with a maximum at $405 \mathrm{~nm}$. The position of the emission maximum and the relative fluorescence intensity significantly depend on the solvent. Thus, in $\mathrm{CH}_{2} \mathrm{Cl}_{2}$, 343-OAc emits green light with a maximum at $530 \mathrm{~nm}$ (large Stokes 
shift of $125 \mathrm{~nm}$ ) and a QY of $15 \%$. In $\mathrm{MeOH}$, the fluorescence maximum is shifted to 571 $\mathrm{nm}$ indicating an enormous Stokes shift of $166 \mathrm{~nm}$. However, due to a very low fluorescence QY in $\mathrm{MeOH}$ (and presumably in other polar solvents), pyridocoumarin 343-OAc is not particularly useful. Another interesting feature of 343-OAc is emission of light in the solid-state upon irradiation of the powdered 343-OAc with a $365 \mathrm{~nm}$ UV lamp. Similarly to pyridocoumarin 343-OAc, compound 349 exhibited a very low QY in $\mathrm{MeOH}$. Transition from pyridocoumarin 343-OAc to pyridonocoumarin 344 resulted in bathochromic shifts of 17-22 $\mathrm{nm}$ and hypsofluoric shifts of 33-47 nm (depending on the solvent). As a result, Stokes shifts displayed by compound 344 turned out to be smaller than the Stokes shift observed for compound 343-OAc and closer to values typical for coumarins $(75-100 \mathrm{~nm})$. The relative fluorescence intensity of $\mathbf{3 4 4}$ showed a pronounced dependence on the nature of solvent. In polar $\mathrm{MeOH}$, the fluorescence QY of $\mathbf{3 4 4}$ was only a third of the value found in $\mathrm{CH}_{2} \mathrm{Cl}_{2}$.

Table 11 Spectral properties of pyrido- and (iso)quinolinocoumarins 343-OAc, 344, 349, 355-H, 356, 360, 362, 373 and 378.

\begin{tabular}{|c|c|c|c|c|c|c|}
\hline Compound & $\lambda_{\text {abs,max }}, \mathrm{nm}$ & $\varepsilon, \mathrm{M}^{-1} \cdot \mathrm{cm}^{-1}$ & $\lambda_{\text {em,max }}, \mathrm{nm}$ & $\Delta \lambda, \mathrm{nm}$ & $\Phi_{\mathrm{fl}}$ & Solvent \\
\hline 343-OAc & 405 & 16700 & 530 & 125 & 0.15 & $\mathrm{CH}_{2} \mathrm{Cl}_{2}$ \\
\hline 343-OAc & 405 & 17100 & 571 & 166 & 0.01 & $\mathrm{MeOH}$ \\
\hline 344 & 422 & 11500 & 497 & 75 & 0.56 & $\mathrm{CH}_{2} \mathrm{Cl}_{2}$ \\
\hline 344 & 427 & 18800 & 524 & 97 & 0.20 & $\mathrm{MeOH}$ \\
\hline $347^{\mathrm{a}}$ & 378 & $\mathrm{n} / \mathrm{d}$ & 458 & 80 & 1.00 & $\mathrm{EtOH}$ \\
\hline 349 & 377 & 14200 & 500 & 123 & 0.02 & $\mathrm{MeOH}$ \\
\hline $355-\mathrm{H}^{\mathrm{b}}$ & 366 & 21800 & 447 & 81 & $\mathrm{n} / \mathrm{d}$ & $\mathrm{EtOH}$ \\
\hline 356 & 411 & 16700 & 577 & 166 & 0.11 & $\mathrm{MeOH}$ \\
\hline 360 & 420 & 13200 & 560 & 140 & 0.29 & $\mathrm{MeOH}$ \\
\hline 362 & 467 & 13400 & 608 & 141 & 0.26 & $\mathrm{MeOH}$ \\
\hline
\end{tabular}


373

378
438

453
19300

$\mathrm{n} / \mathrm{d}$
574

617
136

0.27

164

\section{$0.01 \quad$ PBS 7.4}

The quinoline moiety in compounds $\mathbf{3 5 6}$ and $\mathbf{3 6 0}$ shifts the absorption maxima (by 45 and $56 \mathrm{~nm}$ in $\mathrm{MeOH}$, respectively) towards the red region of the visible spectrum relative to their precursor 355-H. The bathochromic effect is more pronounced in the case of compound 360, where the pyridine nitrogen can participate in delocalization of the lone pair of the 7-dimethylamino group. Upon excitation, compounds 356 and 360 emit yellow fluorescence with large Stokes shifts of 166 and $140 \mathrm{~nm}$, respectively. Both compounds 356 and 360 displayed moderate QYs in $\mathrm{MeOH}$, but the emission efficiency of $\mathbf{3 5 6}$ is lower. Isoquinolinocoumarin $\mathbf{3 6 2}$ with a quaternized pyridine nitrogen showed the most red-shifted values of absorption and fluorescence maxima and a large Stokes shift. Despite the presence of a positive charge, compound 362 possessed a satisfactory QY of 26\%. Quinolinocoumarin 373 is a close structural analog of compound $\mathbf{3 6 0}$ and displayed essentially the same values of Stokes shift and QY as compound 360. At the same time, due to the presence of a 2,2,4-trimethyl-1,2-dihydropyridine moiety, the maxima of the absorption and fluorescence spectra were red-shifted by $14-18 \mathrm{~nm}$ relative to $\mathbf{3 6 0}$.

\subsubsection{Synthesis of water-soluble quinolinocoumarin $\mathbf{3 7 8}$ and its spectral properties}

The model coumarin dye $\mathbf{3 7 3}$ displays a good combination of a large Stokes shift and a satisfactory fluorescence QY in $\mathrm{MeOH}$. In addition, due to the presence of the hydroxyl and masked carboxyl groups, it has two sites for further synthetic modifications. Thus, for the preparation of a water-soluble quinolinocoumarin with a primary phosphate residue, coumarin 373 was subjected to phosphorylation using the phosphoramidite method mentioned earlier. In initial experiments, the phosphatylation with $(t \mathrm{BuO})_{2} \mathrm{PNi}_{2} \mathrm{Pr}_{2}$ proceeded rapidly and cleanly. However, the following treatment with $m \mathrm{CPBA}$ at room temperature yielded many products, possibly due to the epoxidation of the $\mathrm{C}=\mathrm{C}$-bond in the quinoline residue and/or formation of the quinoline $N$-oxide. To our delight, when the oxidation procedure was carried out at $-78^{\circ} \mathrm{C}$, phosphorylated product 376 was formed in a good yield (Scheme 22). The subsequent saponification of $\mathbf{3 7 6}$ with aq. $\mathrm{NaOH}$ in $\mathrm{MeOH}$ led to 
compound 377, which was deprotected with TFA in $\mathrm{CH}_{2} \mathrm{Cl}_{2}$, and afforded the watersoluble coumarin 378.


Scheme 22 Synthesis of the water-soluble coumarin 378 decorated with a primary phosphate group: a) $(\mathrm{tBuO})_{2} \mathrm{PNiPr}_{2}, 1 \mathrm{H}$-tetrazole, $\mathrm{CH}_{2} \mathrm{Cl}_{2}, 40^{\circ} \mathrm{C}, 40 \mathrm{~min}$; then $\mathrm{mCPBA}, \mathrm{CH}_{2} \mathrm{Cl}_{2},-78{ }^{\circ} \mathrm{C}, 1 \mathrm{~h}$; b) aq. $\mathrm{NaOH}$, $\mathrm{MeOH}$, r.t., 3 h; c) TFA, $\mathrm{CH}_{2} \mathrm{Cl}_{2}, 0^{\circ} \mathrm{C}, 40$ min.

Solutions of compound $\mathbf{3 7 8}$ in a PBS buffer at $\mathrm{pH} 7.4$ exhibited an absorption with a maximum at $453 \mathrm{~nm}$ (Table 11) and an emission band in the orange-red spectral region with a maximum at $617 \mathrm{~nm}$ (Stokes shift $164 \mathrm{~nm}$ ). Transition from $\mathrm{MeOH}$ to aqueous solutions resulted in a considerable decrease in fluorescence $(<1 \%)$. The possible explanation is that the single phosphate group probably cannot prevent molecules of $\mathbf{3 7 8}$ with a large, planar, extended and hydrophobic framework from the formation of non-fluorescent aggregates. However, we expect that the fluorescence will improve upon conjugation with proteins. For example, it was the case with dye Abberior Star 520SXP which displayed QY of $0.6 \%$ in a free state in aqueous PBS buffer, but its conjugates with antibodies turned out to be much brighter and are successfully used in optical superresolution microscopy.

\subsubsection{Conclusion and outlook}

Coumarins with a pyridine or an (iso)quinoline fragment fused with positions 3 and 4 of the coumarin scaffold have been synthetized using three different synthetic approaches. Pyridocoumarins 343-OAc and 349 did not provide the desired bathochromic shift and an acceptable fluorescence QYs in polar media, despite the large Stokes shifts. Transition to pyridonocoumarin 344 allowed us to improve emission efficiency, but it is still lower than that of commercial dyes Alexa Fluor 430, Atto 425, Atto 430LS and Abberior Star 
440SXP with comparable optical spectra. (Iso)quinolinocoumarins demonstrated large Stokes shifts and moderate fluorescence QYs in $\mathrm{MeOH}$. However, the introduction of a primary phosphate residue did not help to improve emission efficiency of compound $\mathbf{3 7 8}$ in aqueous media. Nevertheless, in a conjugated form, this dye may demonstrate a better emitting performance, and therefore requires further testing.

\subsection{Synthesis of a carborhodol dye}

\subsubsection{Motivation and key structural elements}

Hybrid fluorophores are assembled from fragments which can be considered as parts of traditional fluorescent dyes. Very often, these compounds combine properties and features of the parent dyes. For example, rhodols belong to the xanthene dyes and may be regarded as hybrids of rhodamines and fluorescein (see Figure 48). Spectral properties of fluorescein, the simplest unsubstituted rhodamine $(\mathrm{R}=\mathrm{H})$ and the simplest rhodol $(\mathrm{R}=$ H) are very close $\left(\lambda_{\text {abs,max }} / \lambda_{\text {flu,max }}=485 / 514,496 / 520\right.$ and $494 / 520 \mathrm{~nm}$, respectively, in basic aqueous solutions). For alkyl-substituted rhodols the absorption and fluorescence maxima are situated approximately between the maxima for fluorescein and the corresponding symmetric alkyl-substituted rhodamine. Unlike the parent fluorescein, rhodols are relatively photostable. ${ }^{[155]}$ Under neutral conditions, rhodols do not form zwitterionic species, and therefore are less susceptible to the formation of nonfluorescent spirolactones which are often not desirable. 

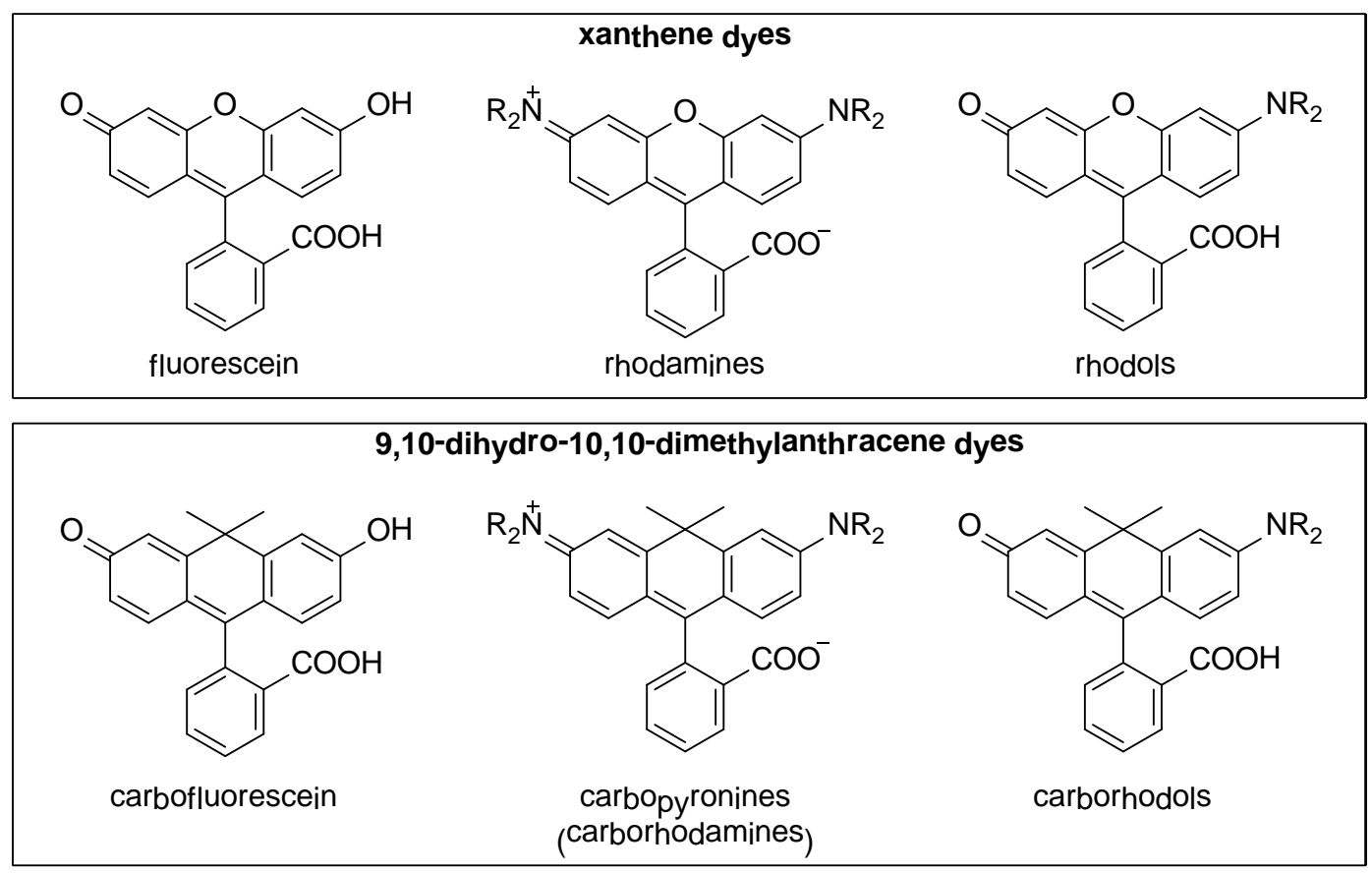

Figure 48 Rhodols and new carborhodol dyes as fluorescein-rhodamine and carbofluorescein-carbopyronine hybrids, respectively.

Other hybrid fluorophores are known. For example, those obtained by "crossbreeding" rhodamines with coumarins. ${ }^{[73]}$ These hybrid dyes inherited certain features from both parent fluorophores, e.g. the emission in the orange-red spectral region from rhodamines and large Stokes shifts which are typical for coumarins.

The replacement of the oxygen atom at C-10 of xanthene dyes with an isopropylidene bridge leads to 9,10-dihydro-10,10-dimethylanthracene dyes. Owing to the positive inductive effect of two methyl groups in the 10-position and the completely new conjugation pattern in the fluorophore, the absorption and fluorescence maxima of carbofluorescein and carborhodamines (carbopyronines) are red-shifted by ca. 50-60 nm relative to the corresponding fluorescein and rhodamines. Following the same pattern as in the case of rhodol dyes, carbofluorescein and carborhodamine may be combined into a completely new fluorophore - carborhodol. This combination is particularly interesting because it enables design of a new, simple and uncharged fluorophore with asymmetric electron density distribution which can lead to a larger Stokes shift and other new properties. Due to the presence of two methyl groups at C-10, the new fluorophore will emit orange-red light. Such red-emitting fluorophores are of particular importance for micros- 
copy applications because the cellular autofluorescence is negligible in this spectral region.

\subsubsection{Synthesis and chemical properties of carborhodol dyes}

The classic strategy for the preparation of carborhodamines (carbopyronines) utilizes addition of an aryllithium species 381 to $N, N, N^{\prime}, N^{\prime}$-tetrasubstituted diaminoanthrone $\mathbf{3 7 9}^{[156]}$ or to carbopyronine $\mathbf{3 8 0}^{[157]}$ (route $a$ in Scheme 23). In one report ${ }^{[156 a]}$ a transformation of carbopyronine 382 into carbofluorescein 383 by alkaline hydrolysis was described. In principle, this reaction must proceed via an intermediate formation of carborhodol 384. However, the hydrolysis rate of compound 384 may exceed that of the starting compound $\mathbf{3 8 2}$ making this method inefficient for the preparation of $\mathbf{3 8 4}$.

After the present work had been finished, an alternative route to carbopyronines was reported $^{[158]}$ (route $b$ in Scheme 23). In this new approach carbofluorescein 385 was first converted to the corresponding triflate 386. The subsequent coupling of $\mathbf{3 8 6}$ with secondary amines according to a Buchwald-Hartwig protocol provided carbopyronines 387 in good yields. As in the case of hydrolysis of 382, the reaction of compound 386 with amines must proceed via intermediate $\mathbf{3 8 8}$ which is, in fact, a carborhodol precursor. Provided that the coupling rates for $\mathbf{3 8 6}$ and $\mathbf{3 8 8}$ are comparable (which seems reasonable because the presence of the remote amino group in compound $\mathbf{3 8 4}$ should not significantly change the electron density at the carbon atom attached to the triflate group in comparison to the starting ditriflate $\mathbf{3 8 6}$ ), the reaction of $\mathbf{3 8 6}$ with one equivalent of the secondary amine could give compound $\mathbf{3 8 8}$ in an acceptable yield. Moreover, in the same report $^{[158]}$ a novel strategy for the synthesis of carbofluorescein 385 was developed. In principle, this method, with some modifications, could allow the synthesis of a carbofluorescein with phenolic hydroxyls protected with two different protective groups. If such an intermediate were available, one of its protected hydroxyl groups could be selectively deprotected and converted to the corresponding triflate. A subsequent Buchwald-Hartwig coupling of the monotriflate could yield a carborhodol precursor which is similar to compound 388, without the formation of carbopyronines as side products.

In our pioneering synthetic approach to carborhodols we decided to use the route $c$ (Scheme 23) which is analogous to the classic synthetic approach to carbopyronines (route $a$ ). In the key step of our approach, aryllithium compound $\mathbf{3 8 1}$ reacts with the 
protected anthrone 389 and yields the protected carborhodol 390. Removal of the protecting group from the phenolic hydroxyl leads to the target carborhodol 391. In contrast to the route $b$, our approach allows introducing tetrahydroisoquinoline and julolidine fragments to the final fluorophore. These structural features "rigidize" the framework of a fluorescent dye and provide better photostability and larger fluorescence QYs, even in polar solvents. Thus, carborhodols with a julolidine fragment are also expected to be photostable and efficient red emitting fluorescent dyes.

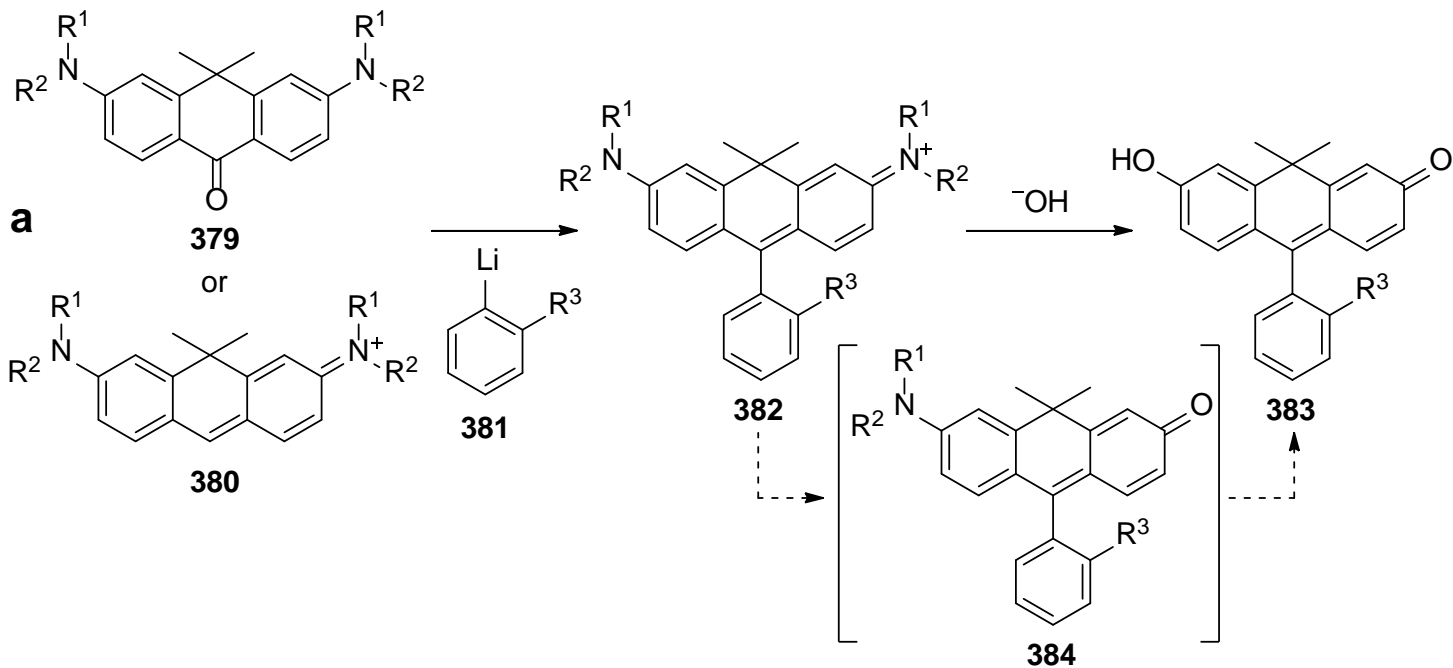

b

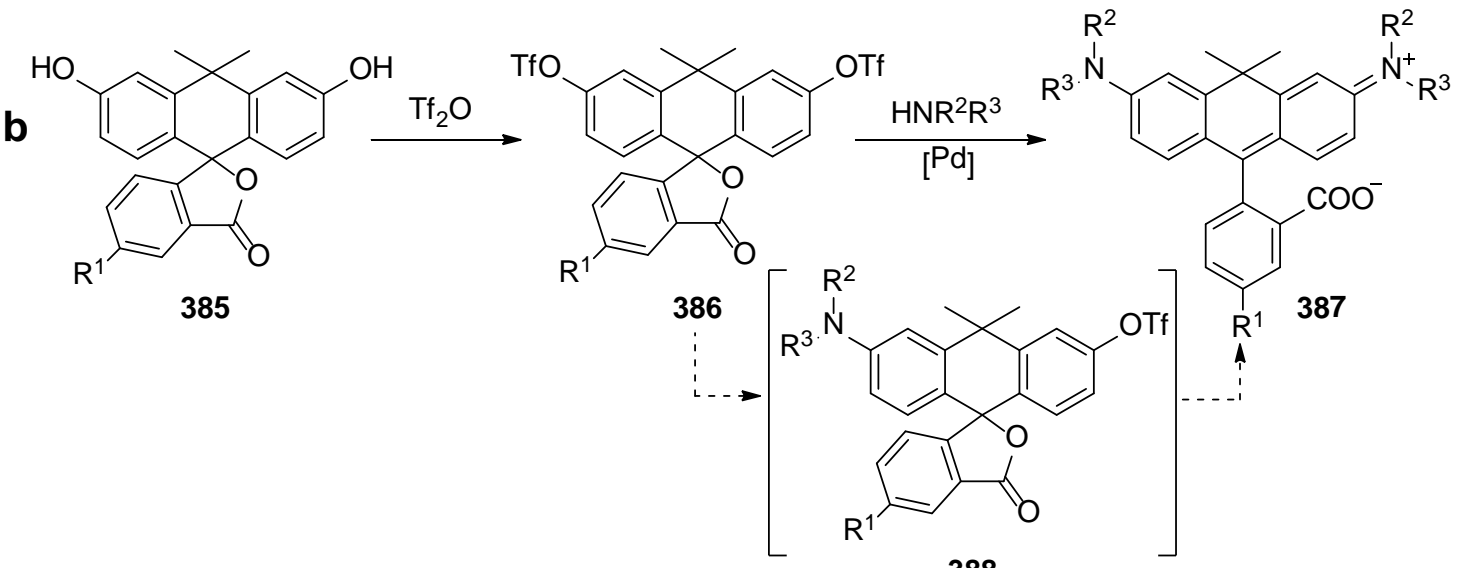<smiles>[R]c1ccccc1CCOc1ccc2c(c1)C(C)(C)c1cc(N([R])C)ccc1C2=O</smiles>

381<smiles>[R]c1ccccc1/C(=C1\C=CC(=[N+]([R1])C)C=C1C(C)(C)C)c1ccc(OC)cc1</smiles>

390

388<smiles>[R][X]Nc1ccc2c(c1)C(C)(C)C1=CC(=O)C=CC1=C2c1ccccc1[R]</smiles>

391

Scheme 23 a) Classic synthetic route to carbopyronines 382 and carbofluoresceins 383 via bis(dialkylamino)anthrones $\mathbf{3 7 9}$ or 9-unsubstituted carbopyronines $\mathbf{3 8 0}$; b) Synthetic approach to carbopyronines based on a Buchwald-Hartwig amination of carbofluorescein triflates 386; c) Synthetic approach to carborhodols used in this work. 
Initially, in order to develop the synthesis of anthrones 389, we were going to use the procedure which worked well for the synthesis of diaminoanthrones 379. ${ }^{[156]}$ To this end, 3-bromo- $N, N$-dimethylaniline 392 was subjected to lithium-bromine exchange, and the resulting aryllithium compound was "quenched" with acetone to yield alcohol 393 (Scheme 24). Subsequent water elimination in boiling chlorobenzene in the presence of $\mathrm{KHSO}_{4}$ provided the building block 394 with an isopropylidene group. Unfortunately, an attempted condensation of 394 with either benzylic alcohol 396 or its protected derivative 397 promoted by $\mathrm{BCl}_{3}$ and PPA did not lead to the desired product 398-R.

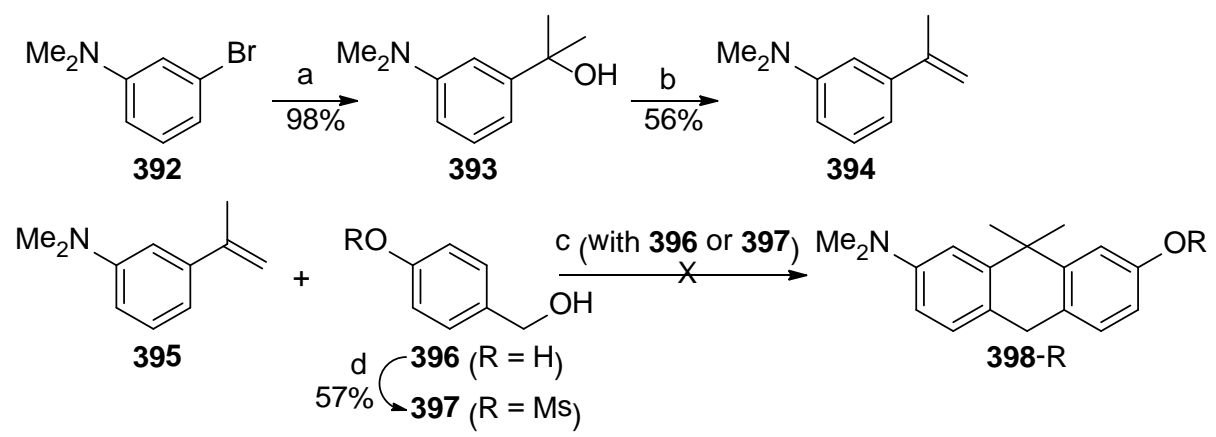

Scheme 24 Attempts to prepare the carborhodol precursor 398-R by a condensation of the building block 395 with 396 or 397: a) BuLi, THF, $-78{ }^{\circ} \mathrm{C}, 20 \mathrm{~min}$; then acetone, $-78{ }^{\circ} \mathrm{C} \rightarrow$ r.t., $1 \mathrm{~h}$; b) $\mathrm{KHSO}_{4}$, chlorobenzene, $140{ }^{\circ} \mathrm{C}, 15 \mathrm{~min}$; c) $\mathrm{BCl}_{3}, \mathrm{CH}_{2} \mathrm{Cl}_{2}, 0^{\circ} \mathrm{C} \rightarrow$ r.t., overnight; then PPA, $\mathrm{H}_{3} \mathrm{PO}_{4}, 110{ }^{\circ} \mathrm{C}, 2 \mathrm{~h}$; d) $\mathrm{MsCl}$, $\mathrm{KOH}, \mathrm{H}_{2} \mathrm{O}, 10 \mathrm{~min}$, r.t.

In a modified approach, 3-bromoanisole 399 was subjected to a Friedel-Crafts acylation with $p$-nitrobenzoyl chloride to afford the benzophenone 400 (Scheme 25). The nitro group in compound $\mathbf{4 0 0}$ was successfully reduced to the primary amino group with $\mathrm{SnCl}_{2}$. $\mathrm{N}$-Methylation of aminobenzophenone $\mathbf{4 0 1}$ was carried out according to a known procedure $^{[159]}$ and provided $N, N$-dimethylaminobenzophenone 404. For the introduction of the isopropanol fragment, that will provide the geminal dimethyl group in the final carborhodol, the carbonyl group in compound $\mathbf{4 0 4}$ has to be protected, for example, as a dimethyl acetal. However, the attempted acetalization using $\mathrm{HC}(\mathrm{OMe})_{3}$ in the presence of montmorillonite $\mathrm{K} 10$ and $\mathrm{TsOH},{ }^{[160]}$ or $\mathrm{CF}_{3} \mathrm{SO}_{3} \mathrm{H}^{[161]}$ failed to provide the required compound. The transformation of benzophenone $\mathbf{4 0 4}$ to the corresponding diaryldichloromethane ${ }^{[162]}$ was successful, but the subsequent reaction with $\mathrm{MeONa}^{[163]}$ did not afford the required dimethyl acetal. Therefore, in order to be able to perform a lithium-bromine exchange, benzophenone $\mathbf{4 0 4}$ was reduced with $\mathrm{NaBH}_{4}$ in the presence of $\mathrm{AlCl}_{3},{ }^{[164]}$ and thus the diarylmethane $\mathbf{4 0 3}$ was obtained. The lithium-bromine exchange in $\mathbf{4 0 3}$ followed 
by a reaction with acetone provided carbinol 402. The cyclization of compound $\mathbf{4 0 2}$ readily occurred in the presence of $\mathrm{AlCl}_{3}$ in $\mathrm{CH}_{2} \mathrm{Cl}_{2} \cdot{ }^{[165]}$ The intermediate 9,10-dihydro10,10-dimethyl derivative (not shown in Scheme 25) represents a leuco-form of the fluorescent dye. It was readily oxidized in air with the formation of colored products, and therefore it was oxidized without isolation directly with $\mathrm{KMnO}_{4}$. As a result, the keto group was restored, and anthrone $\mathbf{4 0 5}$ was formed. Having the important intermediate $\mathbf{4 0 5}$ at hand and using the known methodology, ${ }^{[156 \mathrm{~d}, 157]}$ we easily performed the final transformations. The aryllithium compound 406, which contains the masked carboxylic function, was generated from 2-(2-bromophenyl)-4,4-dimethyl-4,5-dihydrooxazole and tBuLi and introduced to the reaction with ketone $\mathbf{4 0 5}$. During the work-up procedure, a spontaneous ring-opening of the oxazolidinone cycle took place, and ester $\mathbf{4 0 7}$ was formed. The deprotection of the carboxylic group was accomplished by heating in $20 \%$ aq. $\mathrm{HCl}$. These harsh conditions also caused the partial cleavage of the aryl methyl ether, and a mixture of two products $(\mathbf{4 0 8}-\mathrm{Me}$ and $\mathbf{4 0 8}-\mathrm{H})$ was obtained. The carboxyl group in compound 408-Me was activated by $\mathrm{POCl}_{3}$. In the subsequent reaction of the intermediate acid chloride with an excess of the amino ester 413, the required amide $\mathbf{4 1 0}$ was obtained (along with $\mathrm{N}$-methyl-2-pyrrolidone which formed in the course of cyclization of $\mathbf{4 1 3}$ under basic conditions). It is noteworthy that compound $\mathbf{4 0 8}-\mathrm{H}$ can also be amidated according to the method described above (the substitution of the phenolic hydroxyl with a chlorine atom does not occur). The reaction partner 413 was prepared in two steps from $\mathrm{N}$ carboxybenzyl (Cbz) protected 4-aminobutyric acid 411. In the first step, methylation with $\mathrm{MeI}$ in the presence of $\mathrm{Ag}_{2} \mathrm{O}$ provided the Cbz-protected methyl ester 412. Deprotection of the Cbz-protecting group was accomplished by hydrogenation on $\mathrm{Pd} / \mathrm{C}$. In order to prevent the resulting methyl $N$-methylaminobutyrate from a spontaneous cyclization, it was necessary to perform the deprotection step in the presence of $\mathrm{HCl}$. As a result, the hydrochloride $\mathbf{4 1 3}$ was obtained. Demethylation of the methyl ether group in amide 410 with $\mathrm{BBr}_{3}$ was accompanied by the concomitant cleavage of the methyl ester leading to a mixture of compounds $409-\mathrm{Me}$ and $409-\mathrm{H}$. Methyl ester $409-\mathrm{Me}$ could be easily converted to the target dye $\mathbf{4 0 9}-\mathrm{H}$ by saponification of the ester group. The amino-reactive NHS-ester 409-NHS was prepared in MeCN using $N$-hydroxysuccinimide in the presence of HATU and $\mathrm{NEt}_{3}$. Notably, the stability of $\mathbf{4 0 9 - N H S}$ was found to be fairly good: during storage of a sample at $-20{ }^{\circ} \mathrm{C}$ under Ar for about a year, the content of the active ester was still about $70 \%$ according to HPLC. 


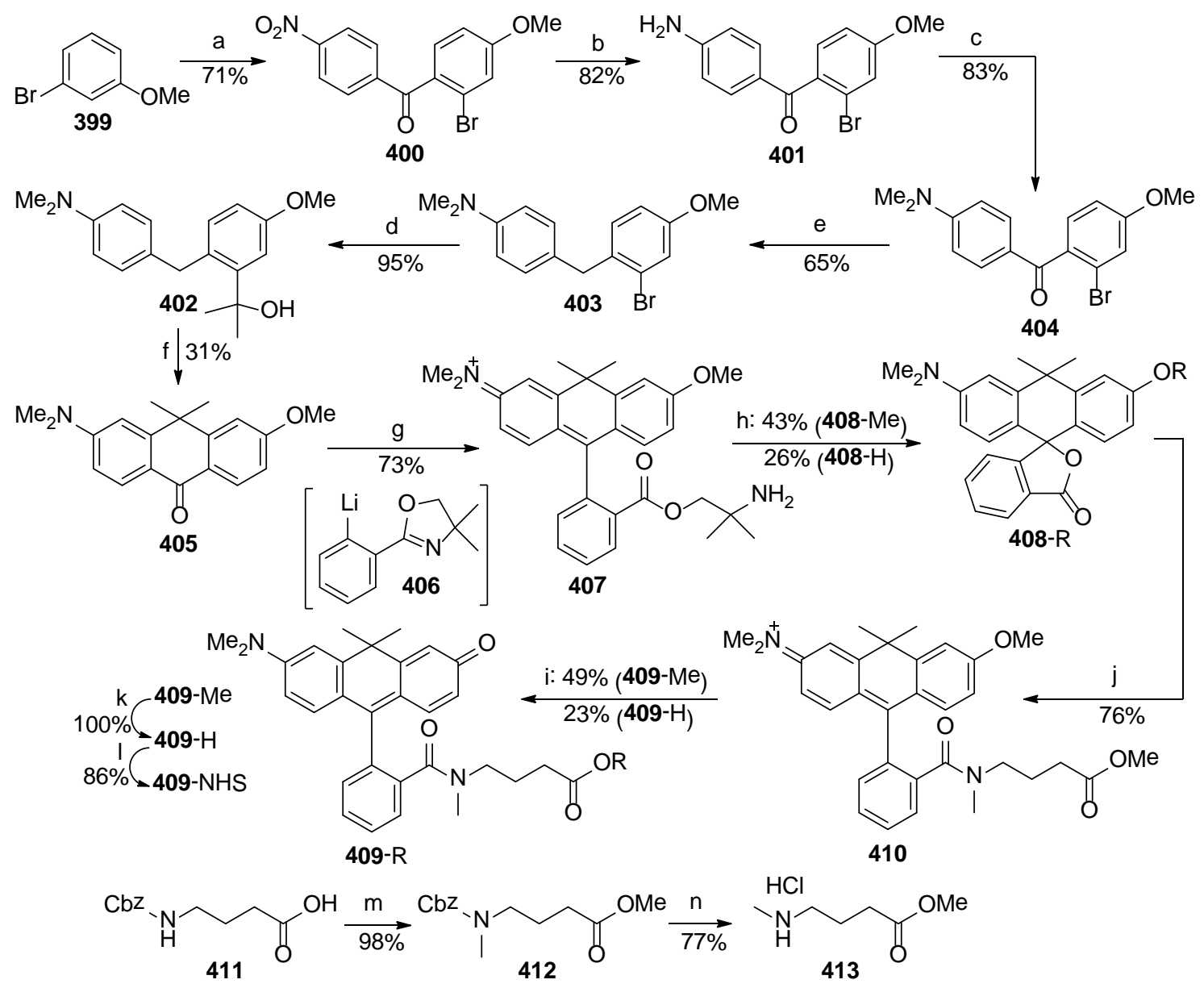

Scheme 25 General approach to carborhodols: a) p-nitrobenzoyl chloride, $\mathrm{AlCl}_{3}, \mathrm{CH}_{2} \mathrm{Cl}_{2}$, reflux, $3 \mathrm{~h}$; b) $\mathrm{SnCl}_{2} \cdot 2 \mathrm{H}_{2} \mathrm{O}, \mathrm{HCl}$, DME, EtOH, r.t., overnight; c) $\mathrm{NaBH}_{3} \mathrm{CN}$, paraform, $\mathrm{AcOH}, 0{ }^{\circ} \mathrm{C} \rightarrow$ r.t., overnight; e) $\mathrm{NaBH}_{4}, \mathrm{AlCl}_{3}$, THF, $0{ }^{\circ} \mathrm{C}$; then reflux, $2 \mathrm{~h}$; d) $\mathrm{BuLi}$, THF, $-78^{\circ} \mathrm{C}, 40 \mathrm{~min}$; then acetone, $-78^{\circ} \mathrm{C} \rightarrow$ r.t.; f) $\mathrm{AlCl}_{3}, \mathrm{CH}_{2} \mathrm{Cl}_{2}, 0{ }^{\circ} \mathrm{C}, 6 \mathrm{~h}$; then r.t., 10 h; then $\mathrm{KMnO}_{4}$, acetone, $-18{ }^{\circ} \mathrm{C}, 2 \mathrm{~h}$; g) 2-(2-bromophenyl)-4,4dimethyl-4,5-dihydrooxazole, THF, $t \mathrm{BuLi},-78{ }^{\circ} \mathrm{C}, 40 \mathrm{~min}$ to form compound 406; then compound 405, THF, $-78{ }^{\circ} \mathrm{C} \rightarrow$ r.t., $1 \mathrm{~h}$; h) aq. $\mathrm{HCl}, 80{ }^{\circ} \mathrm{C}, 6.5 \mathrm{~h}$; j) $\mathrm{POCl}_{3}, \mathrm{ClCH}_{2} \mathrm{CH}_{2} \mathrm{Cl}, 80^{\circ} \mathrm{C}, 2 \mathrm{~h}$; then $413, \mathrm{MeCN}$, $\mathrm{NEt}_{3}$, r.t., 15 min; i) $\mathrm{BBr}_{3}, \mathrm{CH}_{2} \mathrm{Cl}_{2}$, r.t., 1 h; k) aq. $\mathrm{NaOH}$, THF, r.t., 1 h; l) $\mathrm{N}$-hydroxysuccinimide, HATU, $\mathrm{MeCN}, \mathrm{DMF}, \mathrm{NEt}_{3}$, r.t., overnight; m) MeI, $\mathrm{Ag}_{2} \mathrm{O}$, DMF, $0{ }^{\circ} \mathrm{C} \rightarrow$ r.t., overnight; n) $\mathrm{H}_{2}$, $\mathrm{Pd} / \mathrm{C}, \mathrm{EtOH}$, iPrOH, HCl, r.t., 2 h.

We also prepared the similar hybrid dye 416-H with a longer and more hydrophilic linker (Scheme 26). For the preparation of the new linker, the Fmoc-protected amino acid $\mathbf{4 1 4}$ (kindly provided by Dr. Matthias Bischoff) was subjected to a one-pot reductive $\mathrm{N}$ methylation. ${ }^{[166]}$ Deprotection of resulting compound 415-Fmoc in DMF in the presence of piperidine provided $\mathrm{N}$-methylamino acid $\mathbf{4 1 5}-\mathrm{H}$ in an excellent yield. Amidation of compound 408-H with amino acid 415-H afforded carborhodol 416- $\mathrm{H}$. We expected that the hydrophilic linker would improve the imaging performance of the carborhodol by reducing aggregation of the dye molecules in aqueous solutions. Unfortunately, the NHS- 
ester 416-NHS was less stable than 409-NHS, and the quality of the images obtained with its bioconjugates was worse than in the case of $\mathbf{4 0 9}$-NHS.

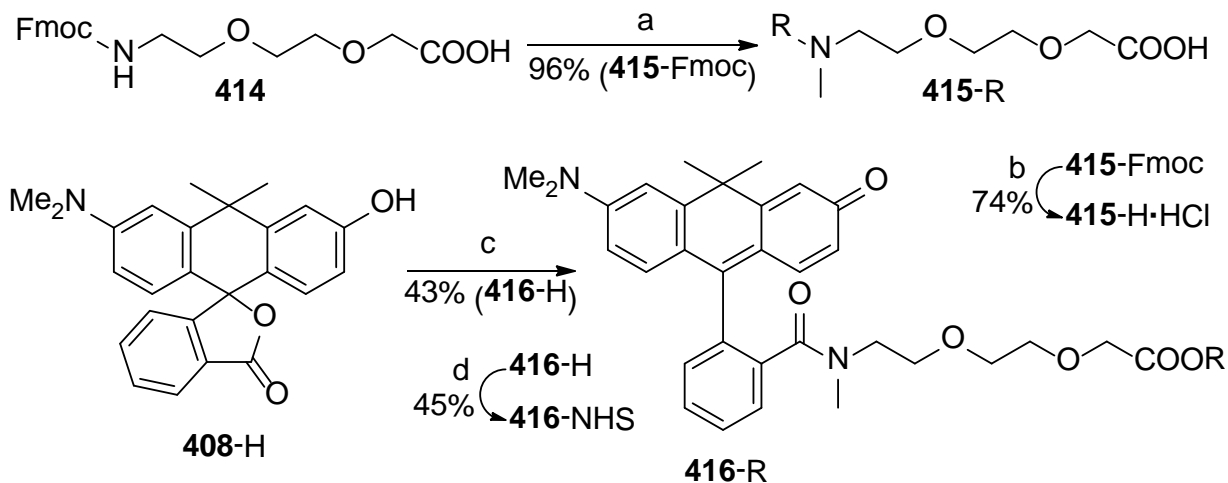

Scheme 26 Synthesis of carborhodol 416- $\mathrm{H}$ with the hydrophilic linker: a) $\mathrm{CH}_{2} \mathrm{O}$, TFA, $\mathrm{CHCl}_{3}, \mathrm{H}_{2} \mathrm{O}, 4{ }^{\circ} \mathrm{C}$ $\rightarrow$ r.t., $30 \mathrm{~min}$; then $\mathrm{Et}_{3} \mathrm{SiH}$, r.t., $30 \mathrm{~min}$; b) piperidine, DMF, r.t., $30 \mathrm{~min}$; then aq. $\mathrm{HCl}$; c) $\mathrm{POCl}_{3}$, $\mathrm{ClCH}_{2} \mathrm{CH}_{2} \mathrm{Cl}, 80{ }^{\circ} \mathrm{C}, 1.5 \mathrm{~h}$; then $415-\mathrm{H} \cdot \mathrm{HCl}, \mathrm{THF}, \mathrm{NEt}_{3}$, overnight; d) $N$-hydroxysuccinimide, HATU, $\mathrm{NEt}_{3}$, MeCN, r.t., overnight.

Fluorinated analogs of fluorescein (for example, Oregon Green) are known to possess significantly better photostability in comparison with the parent fluorophore. At the same time, the influence of fluorine substituents on the positions of absorption and emission maxima, fluorescence QYs and fluorescence lifetimes is rather small. According to Sun et al., ${ }^{[167]}$ the improved resistance to photobleaching may be explained by shorter triplet lifetimes of fluorinated derivatives, which reduce the probability of any photochemical processes, or by a general lower (photo)chemical reactivity of fluorinated compounds in comparison with their analogs without fluorine substituents.

Taking into account these favorable properties of fluorinated fluoresceins, we decided to incorporate fluorine atoms into the carborhodol scaffold. Commercially available 1,2dibromo-3,4,5,6-tetrafluorobenzene 419 (Scheme 27) was considered as a precursor for the fluorescein "half" of the resulting hybrid fluorophore. Selective nucleophilic substitution of the fluorine atom with methoxide anion gave the anisole 420. ${ }^{[168]}$ In compound 420, the bromine atom in the para-position to the methoxy group was selectively replaced with $\mathrm{Li}$, and the resulting organolithium compound was quenched with aqueous EtOH to afford bromide 421. ${ }^{[168 a]}$ Compound 421 represents a fluorinated analog of anisole 399 which was used as a precursor for the synthesis of carborhodol 409-H. However, the same synthetic sequence used for compound $409-\mathrm{H}$ failed in the case of the synthesis of the fluorinated carborhodol. Attempted Friedel-Crafts acylation of anisole 421 with $p$-nitro- 
benzoyl chloride to form the corresponding fluorinated benzophenone did not proceed at all, probably due to a lower chemical reactivity of compound $\mathbf{4 2 1}$ towards electrophiles as compared with compound 399.

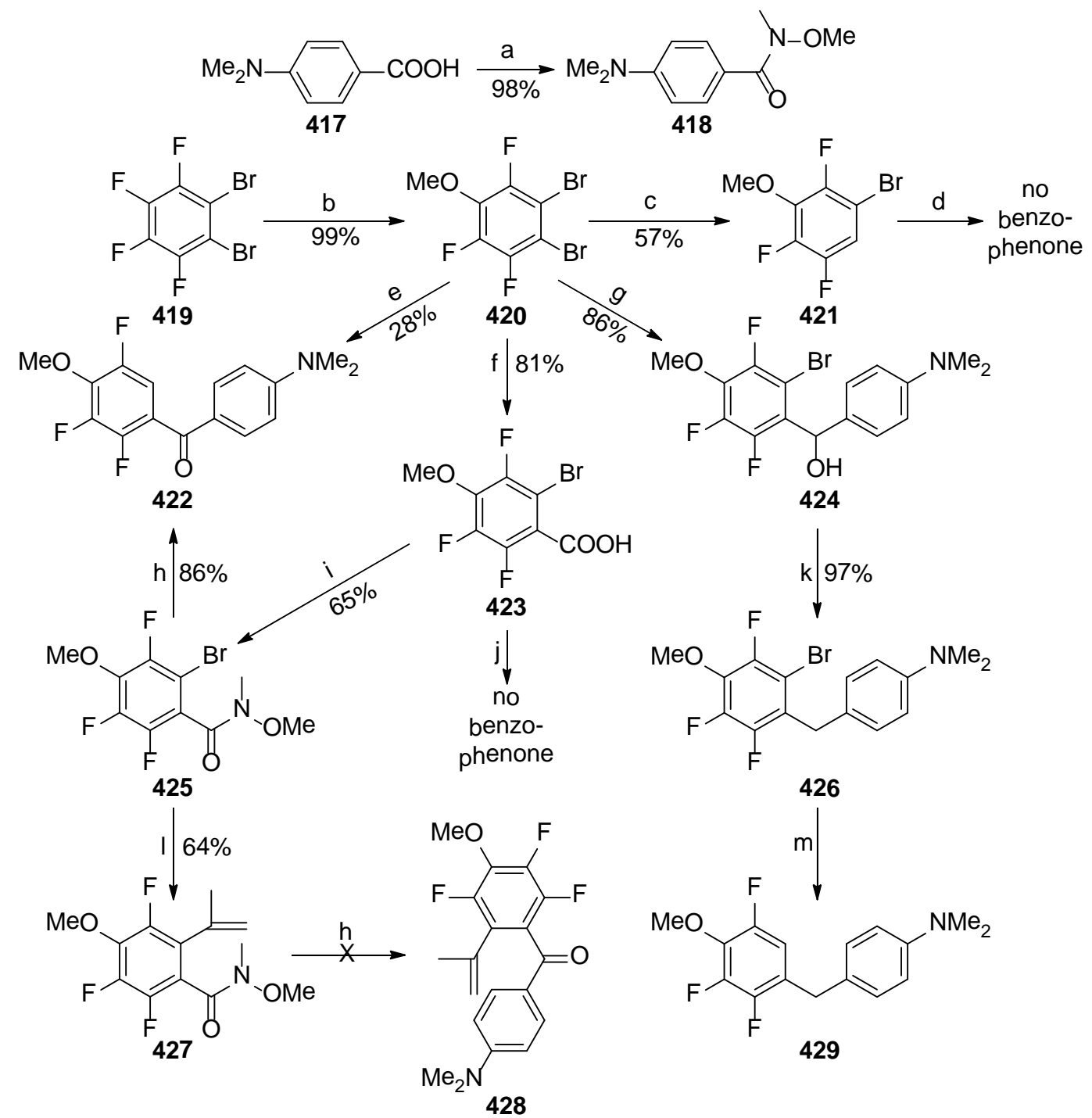

Scheme 27 Attempts to obtain compound 428, a precursor of the fluorinated analogs of hybrid fluorescein carbopyronine dyes: a) triphosgene, $\mathrm{NEt}_{3}, \mathrm{HN}(\mathrm{OMe}) \mathrm{Me} \cdot \mathrm{HCl}, \mathrm{CH}_{2} \mathrm{Cl}_{2}, 0{ }^{\circ} \mathrm{C} \rightarrow$ r.t., 3 h; b) $\mathrm{MeONa}, \mathrm{MeOH}$, reflux, $6 \mathrm{~h}$; c) $\mathrm{BuLi}, \mathrm{Et}_{2} \mathrm{O},-78{ }^{\circ} \mathrm{C}, 4 \mathrm{~h}$; then aq. $\mathrm{EtOH},-78{ }^{\circ} \mathrm{C} \rightarrow$ r.t., overnight; d) $p$-nitrobenzoylchloride, $\mathrm{AlCl}_{3}, \mathrm{ClCH}_{2} \mathrm{CH}_{2} \mathrm{Cl}$, reflux, $3.5 \mathrm{~h}$; e) BuLi, THF, $-78{ }^{\circ} \mathrm{C}, 30 \mathrm{~min}$; then compound $4 \mathbf{4 1 8},-78{ }^{\circ} \mathrm{C}, 30 \mathrm{~min}$; then $0{ }^{\circ} \mathrm{C}, 10 \mathrm{~min}$; f) BuLi, THF, $-78{ }^{\circ} \mathrm{C}, 30 \mathrm{~min}$; then $\mathrm{CO}_{2},-78{ }^{\circ} \mathrm{C} \rightarrow$ r.t.; g) BuLi, $\mathrm{Et}_{2} \mathrm{O},-78{ }^{\circ} \mathrm{C}, 15 \mathrm{~min}$; then $p$ - $N, N$-dimethylaminobenzaldehyde, $-78{ }^{\circ} \mathrm{C} \rightarrow$ r.t., 3 h; h) $p$ - $N, N$-dimethylaminophenylmagnesium bromide, THF, $0{ }^{\circ} \mathrm{C}, 1$ h; then r.t., 1 h; i) triphosgene, $\mathrm{NEt}_{3}, \mathrm{HN}(\mathrm{OMe}) \mathrm{Me} \cdot \mathrm{HCl}, \mathrm{CH}_{2} \mathrm{Cl}_{2}, 0{ }^{\circ} \mathrm{C} \rightarrow$ r.t., 1.5 h; j) $\mathrm{SOCl}_{2}$, DMF, r.t., $1 \mathrm{~h}$; then $N, N$-dimethylaniline, $\mathrm{AlCl}_{3}, \mathrm{CH}_{2} \mathrm{Cl}_{2}, 0{ }^{\circ} \mathrm{C} \rightarrow$ r.t.; then reflux, $6 \mathrm{~h}$; k) $\mathrm{Et}_{3} \mathrm{SiH}$, TFA, $\mathrm{CH}_{2} \mathrm{Cl}_{2}, 40^{\circ} \mathrm{C}, 7 \mathrm{~h}$; l) isopropenylboronic acid, toluene, $\mathrm{Pd}\left(\mathrm{PPh}_{3}\right)_{4}$, aq. $\mathrm{Na}_{2} \mathrm{CO}_{3}$, EtOH, $110{ }^{\circ} \mathrm{C}$, overnight; m) BuLi, THF, $-78^{\circ} \mathrm{C}, 30 \mathrm{~min}$; then acetone, $-78^{\circ} \mathrm{C} \rightarrow$ r.t., $2 \mathrm{~h}$. 
Another ketone synthesis is the reaction of organolithium or organomagnesium compounds with Weinreb amides. ${ }^{[169]}$ To apply this method for the synthesis of the fluorinated benzophenone $\mathbf{4 2 8}$, dibromide $\mathbf{4 2 0}$ was converted with one equivalent of BuLi to the corresponding organolithium compound, which was then acylated with the Weinreb amide 418. In the course of this reaction, the second bromine atom "disappeared”, and although the product - benzophenone $\mathbf{4 2 2}$ - was isolated, it could not be used in further synthetic steps. Interestingly, when the organolithium compound prepared from compound 420 and one equivalent of $\mathrm{BuLi}$, was treated with a stronger electrophile, such as $\mathrm{CO}_{2}$ or $p$ - $N, N$-dimethylaminobenzaldehyde, the corresponding products, benzoic acid $\mathbf{4 2 3}$ and alcohol 424, retained the second bromine. In principle, the acid chloride prepared from compound $\mathbf{4 2 3}$ can serve as an acylation reagent in the Friedel-Crafts reaction with $N, N$-dimethylaniline to give the important benzophenone intermediate. Unfortunately, all our trials to provide the desired product failed. We also prepared the Weinreb amide $\mathbf{4 2 5}$ and treated it with $p$ - $N, N$-dimethylaminophenylmagnesium bromide, and again, the bromine atom was "lost" in the course of the reaction. Ionic hydrogenation ${ }^{[170]}$ of alcohol 424 with $\mathrm{Et}_{3} \mathrm{SiH}$ and TFA provided the diarylmethane 426, the important intermediate analogous to compound 403. To our regret, in the next synthetic step, the lithium-bromine exchange followed by reaction with acetone yielded debrominated compound $\mathbf{4 2 9}$ as a main product.

In the search for new synthetic methods which do not involve organometallic reagents, we turned our attention towards Pd-catalyzed reactions. In this respect, isopropenylboronic acid can be used for the introduction of the isopropenyl group which will later form the isopropylidene bridge at C-10 of the final carborhodol scaffold. Thus, in a Suzuki reaction of compound $\mathbf{4 2 5}$ with isopropenylboronic acid, coupling product $\mathbf{4 2 7}$ was successfully obtained. However, the subsequent reaction of amide 427 with $p-N, N$ dimethylaminophenylmagnesium bromide yielded only traces of the benzophenone $\mathbf{4 2 8}$.

\subsubsection{Properties and imaging performance of carborhodol dyes}

Table 12 presents the most important photophysical properties of carborhodol dyes $408-\mathrm{H}$ and $409-\mathrm{H}$, their precursor $409-\mathrm{Me}$, conjugates of $409-\mathrm{H}$ with antibodies and the related data for compound KK114, ${ }^{[171]}$ which was used together with $\mathbf{4 0 9 - H}$ in two-color imaging experiments. 
Table 12 Photophysical properties of carborhodol dyes $\mathbf{4 0 8}-\mathrm{H}$ and $\mathbf{4 0 9}-\mathrm{H}$, their precursor $\mathbf{4 0 8}$-Me and dye KK114 at room temperature

\begin{tabular}{|c|c|c|c|c|c|c|c|}
\hline Compound & $\lambda_{\text {abs,max }}, \mathrm{nm}$ & $\varepsilon, \mathrm{M}^{-1} \cdot \mathrm{cm}^{-1}$ & $\lambda_{\mathrm{em}, \max }, \mathrm{nm}$ & $\Delta \lambda, \mathrm{nm}$ & $\Phi_{\mathrm{fl}}$ & $\tau^{\mathrm{a}}, n s$ & Solvent \\
\hline 408-Me & 513,549 & 18250,18430 & 585 & 72,36 & 0.01 & - & $\mathrm{MeOH}^{\mathrm{b}}$ \\
\hline 408-H & 515, 553 & 30000,37400 & 585 (broad) & 70,32 & 0.11 & - & $\mathrm{MeOH}^{\mathrm{b}}$ \\
\hline 408-H & 560 & 34300 & 598 & 38 & 0.94 & - & $\mathrm{MeOH}^{\mathrm{c}}$ \\
\hline 409-H & 573 (broad) & 41000 & 613 & 40 & 0.64 & 4.0 & $\mathrm{MeOH}$ \\
\hline 409-H & 586 & 59000 & 613 & 27 & 0.32 & 2.5 & $\mathrm{PBS}^{\mathrm{d}}$ \\
\hline $409-\mathrm{H} \cdot \mathrm{AB}^{\mathrm{e}}$ & 586 (broad) & - & 613 & 27 & 0.39 & 1.3 & $\mathrm{PBS}^{\mathrm{d}}$ \\
\hline $409-H \cdot A B^{f}$ & 586 (broad) & - & 613 & 27 & 0.09 & 1.6 & $\mathrm{PBS}^{\mathrm{d}}$ \\
\hline KK114 & 636 & 90000 & 660 & 24 & 0.53 & 3.6 & $\mathrm{PBS}^{\mathrm{d}}$ \\
\hline $\mathrm{KK} 114 \cdot \mathrm{AB}$ & 636 & - & 660 & 24 & 0.40 & 3.6 & $\mathrm{PBS}^{\mathrm{d}}$ \\
\hline
\end{tabular}

${ }^{a}$ Lifetime of the excited state $\mathrm{S}_{1} .{ }^{\mathrm{b}}$ With $0.1 \% \mathrm{v} / \mathrm{v}$ TFA. ${ }^{c}$ With $0.1 \% \mathrm{v} / \mathrm{v} \mathrm{NEt} 3 .{ }^{\mathrm{d}}$ Phoshate-buffered saline, $\mathrm{pH}$ 7.4. ${ }^{\mathrm{e} C o n j u g a t e}$ with sheep-antimouse antibody, degree of labeling (DOL): $5.2 .{ }^{\mathrm{f}}$ Conjugate with goat-antirabbit antibody, DOL: 13.

Like fluorescein, ${ }^{[172]}$ methylcarborhodol 408-Me and carborhodol 408-H (along with their spirolactone form) may exist in a complex equilibrium between zwitter-ion 430-R, anion 431 (only for 408-H), cation 432-R, and quinoid 433 (only for $408-\mathrm{H}$, Scheme 28a). The position of this equilibrium is influenced by the polarity and acidity of the environment. In neutral $\mathrm{MeOH}$, compounds $\mathbf{4 0 8}-\mathrm{Me}$ and $\mathbf{4 0 8}-\mathrm{H}$ predominantly exist in their "closed" spirolactone forms, and therefore, their solutions are almost colorless with a slight red tint which may be caused by small concentrations of open forms 430-R and/or 433. Upon acidification with TFA, the solutions readily become red-colored, which may correspond to the formation of the "open” protonated forms 432-R. Absorption and emission spectra of 432-Me and 432- $\mathrm{H}$ in acidic $\mathrm{MeOH}$ were found to be almost identical. Thus, in the absorption spectra two maxima around 515 and $550 \mathrm{~nm}$ with comparable intensities were observed, whereas emission spectra of both compounds displayed a maximum at $585 \mathrm{~nm}$. The fluorescence QY of 431-H turned out to be significantly higher than that of compound 431-Me. A similar trend was observed in rhodol dyes: conversion of the $O$-substi- 
tuted rhodols to $O$-free dyes induced a drastic increase in the fluorescence intensity. ${ }^{[173]}$ Upon addition of $0.1 \% \mathrm{v} / \mathrm{v} \mathrm{NEt}$ to the methanolic solution of $408-\mathrm{Me}$, no color change occurred. In contrast, under the same conditions, compound 408-H formed the redcolored deprotonated "open” form 430-H which displayed intense fluorescence in the orange-red region of the visible spectrum $\left(\lambda_{\mathrm{em}, \max }=598 \mathrm{~nm}, \Phi_{\mathrm{fl}}=0.94\right)$. Carborhodol 409-H cannot form a spirolactone or spirolactame ring and, therefore, under neutral and basic conditions exists in the open quinoid form (Scheme 28b). Maxima of its absorption and emission spectra in $\mathrm{MeOH}$ are red-shifted by 13-15 $\mathrm{nm}$ in comparison with carborhodol 408-H. Upon transition to aqueous media, the absorption maximum of compound 409-H undergoes a further bathochromic shift of $13 \mathrm{~nm}$, whereas the maximum position of the emission spectrum stays nearly unchanged. Interestingly, in $\mathrm{MeOH}$, the extinction coefficient is lower than in PBS buffer, but owing to the broader band, the oscillator strength is approximately the same. Remarkably, the absorption band of antibody conjugates $409-\mathrm{H} \cdot \mathrm{AB}$ is broadened as well.

The fluorescence QY of the free dye 409-H in PBS buffer (0.32) was found to be a factor of two lower than in $\mathrm{MeOH}(0.64)$. QYs of the antibody conjudates depend on the degree of labeling (DOL) which shows the average amount of the dye residues attached to one antibody molecule with $\mathrm{M} \approx 150000$. Thus, the antibody conjugate with a relatively high DOL (5.2) displayed a QY of 39\% in aqueous PBS buffer. As the values of DOL reached 13 and 15, the QYs decreased to 9 and 4\%, respectively. For the highest imaging brightness, it is necessary to maximize the values of $\varepsilon \times D O L \times \Phi_{\mathrm{fl}}$. In this respect, the conjugates of $\mathbf{4 0 9}-\mathrm{H}$ with DOL $=5.2$ are advantageous (provided that the extinction coefficient $\varepsilon$ for the dye $\mathbf{4 0 9}-\mathrm{H}$ is the same in the free state and in conjugates).

Another important feature of the new carborhodol dye is the shortened fluorescence lifetimes $\tau$ in the conjugated state. Thus, the lifetimes were found to be $1.2-1.6 \mathrm{~ns}$, whereas dye KK114 displays constant $\tau$ values in the range of 3.3-3.7 ns. ${ }^{[111 b]}$ The difference in lifetimes can be used in multilifetime $\mathrm{STED}^{[174]}$ to discriminate between two proteins labeled with dyes 409-H and KK114. 


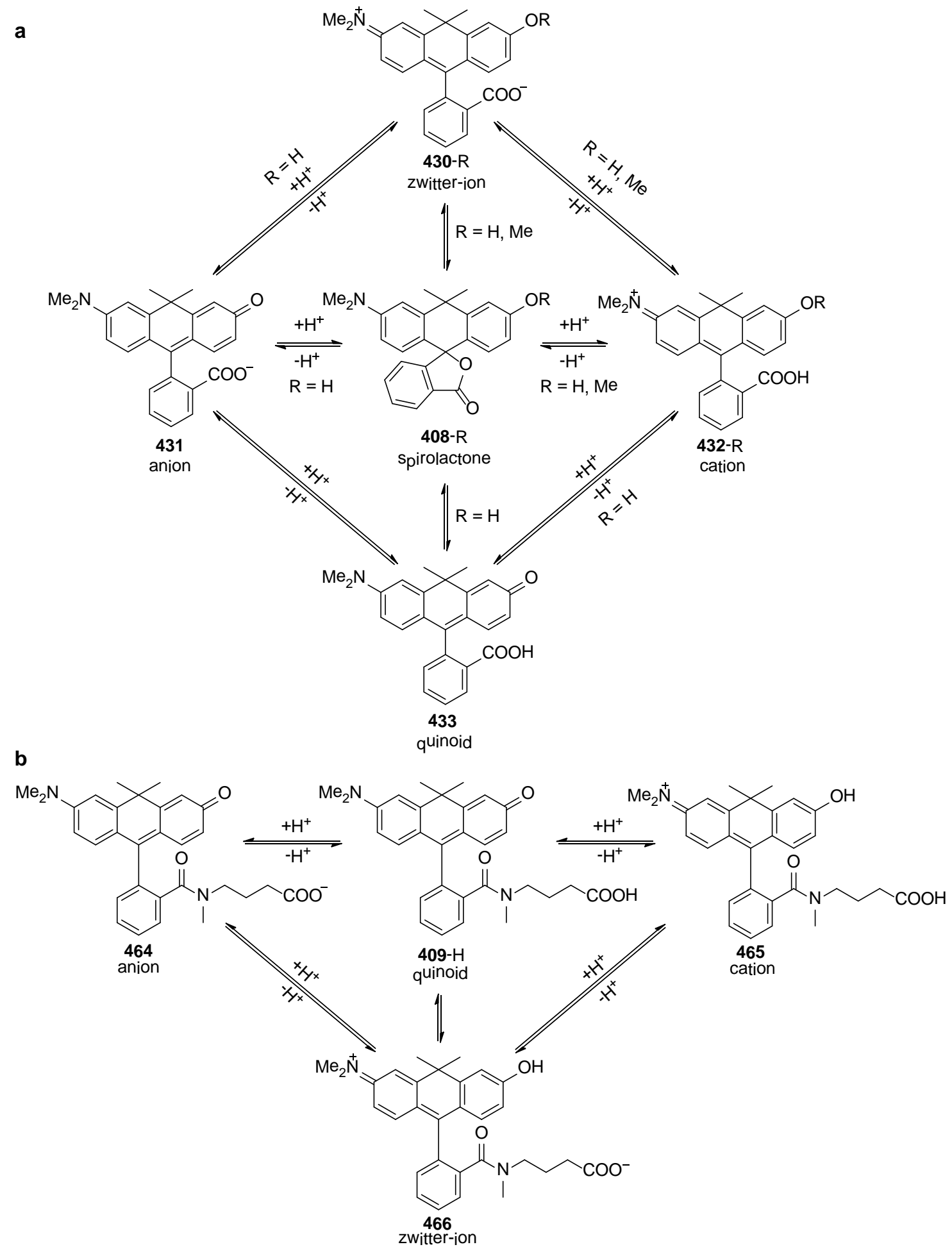

Scheme 28 a) The acid-base equilibrium between the colorless "closed" forms of compounds 408-R and colored "open" forms 430-R, 431, 432-R and 433. b) The acid-base equilibrium between neutral and charged forms of compound $409-\mathrm{H}$.

The Stokes shift of carborhodol 409-H in aqueous PBS was found to be relatively small $(27 \mathrm{~nm})$. However, due to the broad absoption and emission bands, the "effective" value 
is higher. In other words, the hybrid dye 409-H can be efficiently excited with green light (for example, with the $514 \mathrm{~nm}$ line of an argon laser), while the emitted light is red (Figure 49). This remarkable and useful feature is not typical for the rhodamine and carbopyronine dyes and enables use of carborhodol $409-\mathrm{H}$ in two-color imaging. As a second dye, we used a bright and photostable near-emitting fluorescent marker KK114 (for its chemical structure, see ref. ${ }^{[171]}$ ). The main spectral parameters of KK114 are given in Table 12 and Figure 49. Conjugates of KK114 with antibodies exhibited essentially the same absorption and emission spectra as KK114 in the free state, except for a very small red-shift of the absorption band. Owing to a substantial overlap of the emission spectra of carborhodol 409-H and the benchmark dye KK114, the same detection channel and the same STED laser can be used for both fluorophores.
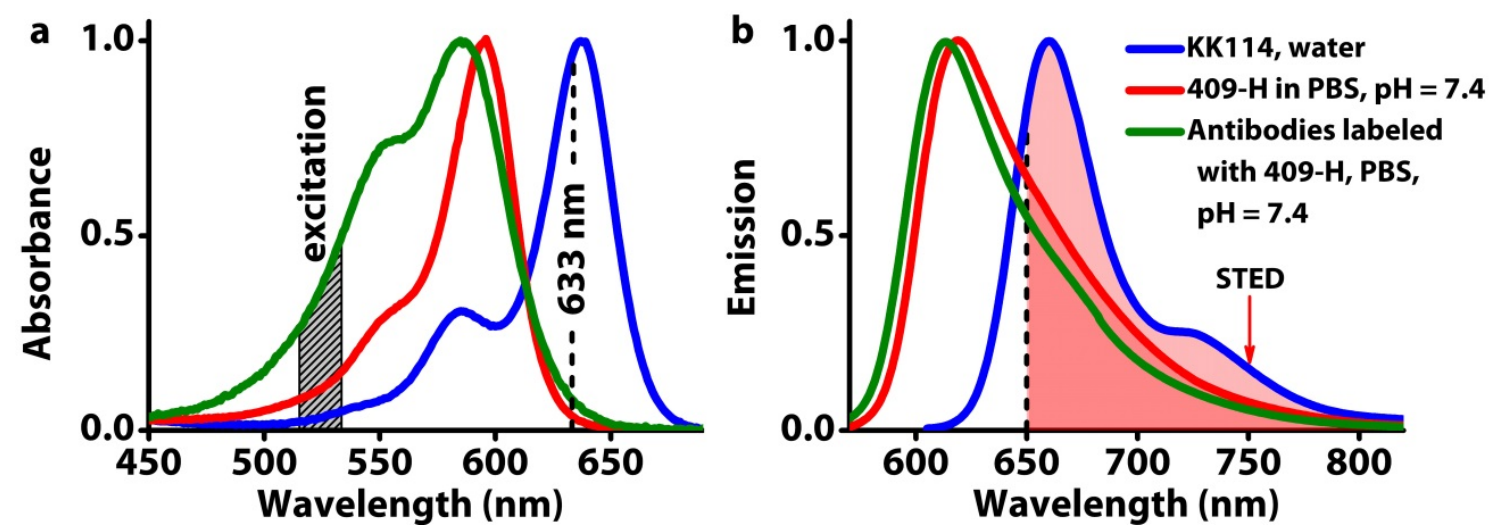

Figure 49 Normalized absorption (a) and emission (b) spectra of compound 409-H, its conjugate with goat anti-rabbit antibody, and the reference dye KK114. Excitation regions $(514-532 \mathrm{~nm}$ and $633-640 \mathrm{~nm}$, respectively), detection area $(650-800 \mathrm{~nm})$ and a STED wavelength $(750 \mathrm{~nm})$ are shown.

In multicolor imaging the crosstalk observed in the course of the excitation with different light sources has to be low. To evaluate the crosstalk between two excitation channels, mammalian tubulin was labeled with compound $409-\mathrm{H}$ or ATTO594 and KK114 dyes (Figure 50). Imaging was performed in a confocal microscope with two excitation lasers (514 and $633 \mathrm{~nm}$ ), and emission was collected beyond $650 \mathrm{~nm}$. Mowiol was used as an embedding medium. KK114 displayed a relatively low level of crosstalk upon excitation at $514 \mathrm{~nm}(10-15 \%)$. Atto594 showed a high crosstalk (up to 60\%) in the "KK114 channel”, while 409-H proved to have negligible crosstalk as expected. This result shows that the dye pair 409-H/KK114 provides much lower crosstalk than the ATTO594/KK114 pair, and therefore, may be advantageously used in two-color imaging and colocalization studies even without linear unmixing or other image processing techniques. 

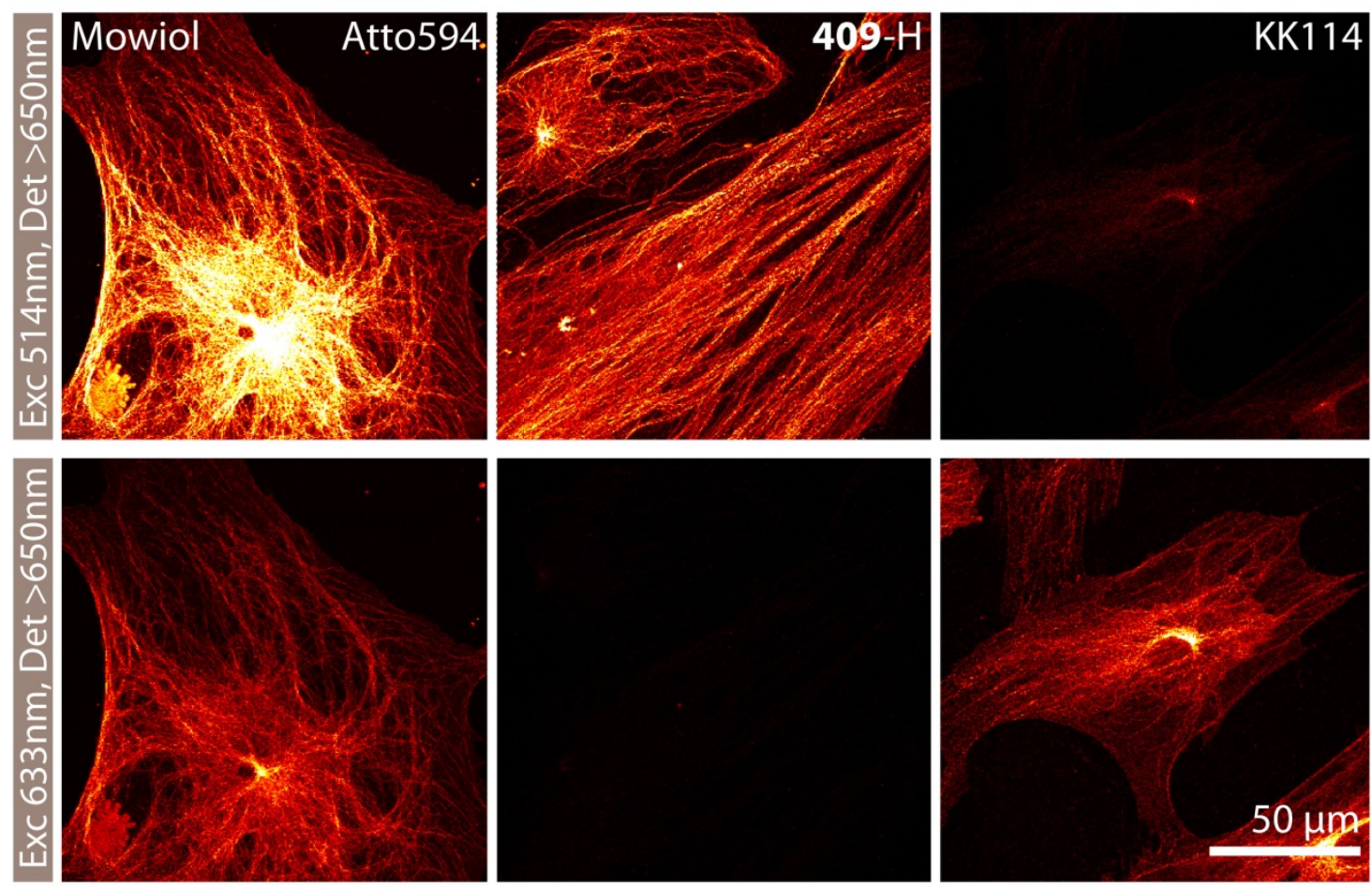

Figure 50 Immunofluorescence imaging and comparison of the crosstalk observed between compound 139$\mathrm{H}$ or ATTO594, and KK114 dyes in mowiol, two excitation sources (514 and $613 \mathrm{~nm}$ ), and one detection channel $(650-750 \mathrm{~nm})$.

Figure 51 shows fluorescence images of cells labeled with $409-\mathrm{H}$ (mitochondria) and KK114 (peroxysomes) and embedded in Mowiol. Although some crosstalk is visible in "KK114", both objects are well discernible. 

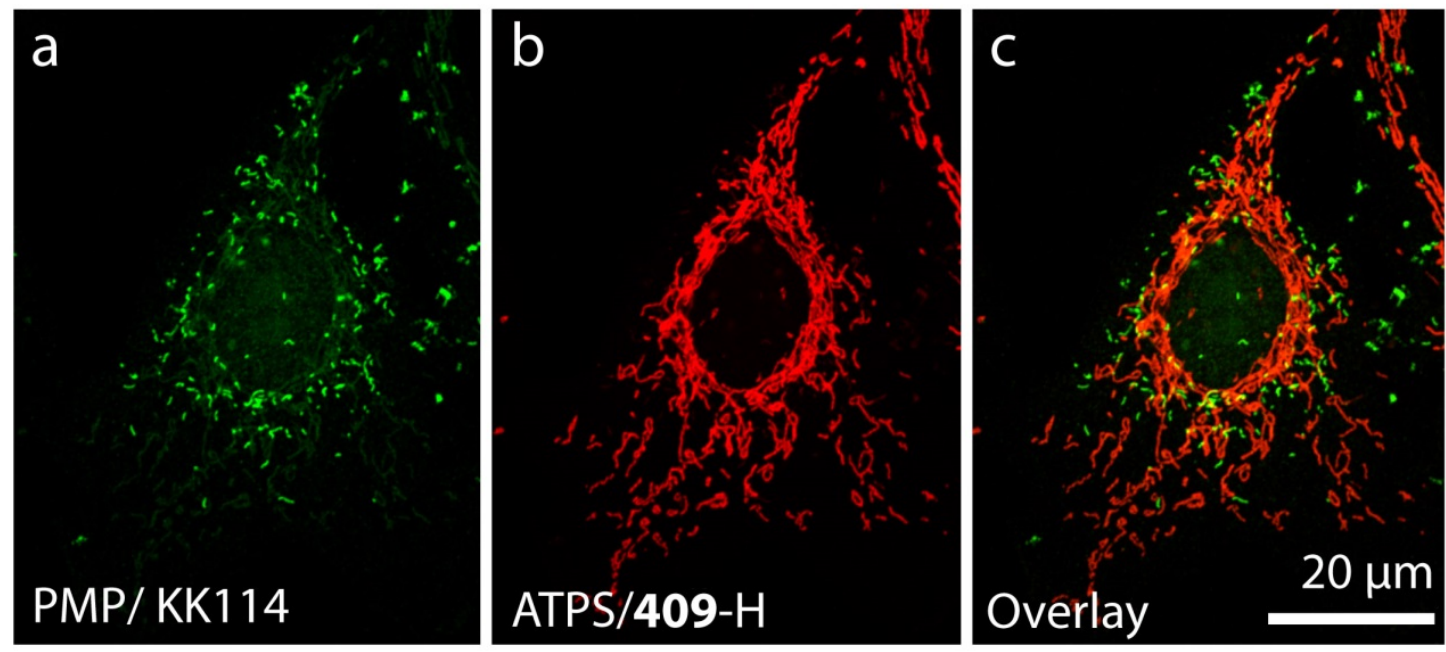

Figure 51 Dual color imaging with dyes 409-H and KK114. A subunit of the mitochondrial ATP synthase and the peroxysomal protein PMP70 were labeled in Vero cells using dyes 409-H and KK114, respectively. Imaging was performed in a custom-made microscope; excitation with laser light at $640 \mathrm{~nm}$ (a) and $532 \mathrm{~nm}$ (b), respectively; confocal detection between 650 and $690 \mathrm{~nm}$. (c) Overlay of (a) and (b). To discriminate the structures, the pictures in the "KK114 channel” and "409-H channel" are pseudocolored in red and green, respectively.

To test the new carborhodol in super-resolution STED microscopy, the vimentin cytoskeleton in Vero cells was immunolabeled with compound 409-H. Imaging was performed in a custom-built STED microscope with excitation at $532 \mathrm{~nm}(\sim 40 \mu \mathrm{W})$ and a STED irradiation at $760 \mathrm{~nm}(\sim 200 \mathrm{~mW})$. The confocal detection was carried out in the 650-690 $\mathrm{nm}$ range. As visible in Figure 52, the performance of carborhodol 409-H is very good, even though the STED wavelength is shifted by more than $140 \mathrm{~nm}$ to the red from the emission maximum. The optical resolution achieved with dye 409- $\mathrm{H}$ was about $80 \mathrm{~nm}$. At the same time, the best resolution of this STED microscope obtained with different dyes was in the range of 40-50 nm. The reason for lower STED resolution with 409-H might be photobleaching. Although we expected carborhodol 409- $\mathrm{H}$ to be more photostable than fluorescein, its photostability apparently cannot reach the values typical for rhodamine dyes. Under STED conditions compound 409-H bleaches significantly faster than rhodamine dye KK114, which is considered to be one of the best STED dyes. ${ }^{[156 d]}$ Trying to improve the photostability of carborhodol dyes, we planned to replace three hydrogen atoms in the fluorescein "half" of the molecule with fluorine. Similar structural modifications applied to the fluorescein resulted in a series of Oregon Green dyes which showed significantly better resistance against photobleaching. ${ }^{[167]}$ Although

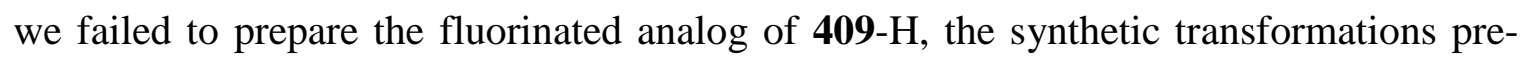


sented in Scheme 27 may be useful for the design of other fluorinated 10,10dimethylanthracene-9(10H)-ones.


Figure 52 STED imaging with compound 409- $\mathrm{H}$. The vimentin cytoskeleton of mammalian cells was labeled with compound 409-H. Top: confocal and STED images of the same region of the sample. Bottom: close-ups of the boxed regions in the upper panels.

\subsubsection{Conclusion and outlook}

A general synthetic approach to new hybrid dyes - carborhodols - has been developed. The new dyes provided bright protein conjugates and low crosstalk in two-color imaging when used together with the benchmark red-emitting dye KK114. Another remarkable feature is the relatively short lifetime in the conjugated form. This allows the use of carborhodols in multilifetime microscopy experiments with other dyes possessing longer lifetimes. The synthetic route to carborhodols given in Scheme 25 can be used for the preparation of structurally diverse dyes with extended conjugation (for example, compounds with 1,2-dihydro-1,2,2,4-tetramethylpyridine fragments). However, the necessity of lithium-halogen exchange at the two key steps restricts the synthetic freedom. Another 
prospective piece of research is the future evaluation of the cell permeability of carborhodol 409-H and its derivatives. 


\section{Experimental part}

\subsection{General remarks}

UV-visible absorption spectra were recorded on a Varian Cary $4000 \mathrm{UV}$-Vis spectrophotometer, and the fluorescence spectra on a Varian Cary Eclipse fluorescence spectrophotometer. The fluorescence quantum yields were determined by comparison with the reference dyes with the known quantum yields (for a detailed description, see: www.horiba.com/fileadmin/uploads/Scientific/Documents/Fluorescence/quantumyieldstr ad.pdf). A MICROTOF spectrometer equipped with ESI ion source Apollo and direct injector with LC autosampler Agilent RR 1200 was used for obtaining high resolution mass spectra (ESI-HRMS). ESI-HRMS were obtained also on APEX IV spectrometer (Bruker). HPLC system (Knauer): Smartline pump 1000 (2×), UV detector 2500, column thermostat $4000\left(25^{\circ} \mathrm{C}\right)$, mixing chamber, injection valve with 20 and $100 \mu \mathrm{L}$ loop for the analytical and preparative columns, respectively; 6-port-3-channel switching valve; analytical column: Eurospher-100 C18, $5 \mu \mathrm{m}, 250 \times 4 \mathrm{~mm}, 1.1 \mathrm{~mL} / \mathrm{min}$; solvent A: water + $0.1 \mathrm{v} / \mathrm{v} \%$ TFA; solvent B: MeCN + $0.1 \mathrm{v} / \mathrm{v} \%$ TFA; detection at $254 \mathrm{~nm}$ or as specified. Analytical TLC was performed on MERCK ready-to-use plates with regular silica gel 60 (F254) and UV-detector (unless specified otherwise). Preparative column chromatography was performed on silica gel $60(40-63 \mu \mathrm{m})$ from Macherey-Nagel (Germany). NMR device: Varian (Agilent) 400-MR (400 MHz) unless specified otherwise. Coupling constants $(J)$ are given in Hz. In the APT mode, the ${ }^{13} \mathrm{C}$ signals of the methyl $\left(\mathrm{CH}_{3}\right)$ and methyne $(\mathrm{CH})$ groups are "positive” $(+)$, while the signals of methylene groups $\left(\mathrm{CH}_{2}\right)$ and carbons without attached hydrogen atoms are "negative” (-). Reactions were carried out upon magnetic stirring in Schlenk flasks equipped with septa or reflux condensers with bubble counters under argon using a standard manifold with vacuum and argon lines. Freeze-drying of the dye solutions in aqueous acetonitrile was performed with ALPHA 2-4 LD plus device with the cooler maintained at $-80{ }^{\circ} \mathrm{C}$ (Martin Christ, Germany). 


\subsection{Experimental procedures}<smiles>CS(=O)O[Ga+]c1cccc(N)c1</smiles>

\section{3-[(tert-Butyldimethylsilyl)oxy]aniline (286-TBDMS)}

Compound 286 was prepared according to the published procedure::175] 3-Aminophenol (21.8 g, $0.200 \mathrm{~mol}$ ) and imidazole (34 g, $0.50 \mathrm{~mol}$ ) were dissolved in DMF (200 mL), the solution was cooled with an ice-water bath, and TBDMSCl (36.1 g, $0.24 \mathrm{~mol}$ ) was added in one portion. The cooling bath was removed, the reaction mixture was allowed to warmup to room temperature, and stirred for $1 \mathrm{~h}$. DMF (ca. $150 \mathrm{~mL}$ ) was evaporated in vacuo at $55{ }^{\circ} \mathrm{C}$, the residue was diluted with EtOAc $(250 \mathrm{~mL})$, washed with sat. aq. $\mathrm{NaHCO}_{3}$ (twice), water (several times), brine and dried over $\mathrm{MgSO}_{4}$. After evaporation of solvents, the oily residue was dried in vacuo (0.5 Torr) to a constant weight. Purification by column chromatography (gradient elution with $n$-hexane to $n$-hexane/ $\mathrm{Et}_{2} \mathrm{O}=5 / 1$ ) afforded compound 286-TBDMS $\left(R_{\mathrm{f}}=0.24\right.$ in $n$-hexane/Et $\left.{ }_{2} \mathrm{O}=8 / 1\right)$ as a clear oil (34.1 g, 76\%). ${ }^{1} \mathrm{H}$ NMR (300 MHz, $\mathrm{CDCl}_{3}$ ): $\delta=0.21$ (s, $6 \mathrm{H}, 2 \times \mathrm{Me}$ ), 0.99 (s, $9 \mathrm{H}, t \mathrm{Bu}$ ), 3.60 (br. s, $2 \mathrm{H}$, $\mathrm{NH}_{2}$ ), 6.18 (m, $\left.1 \mathrm{H}, \mathrm{Ar}\right), 6.25$ (m, $\left.2 \mathrm{H}, \mathrm{Ar}\right), 6.98$ (m, $\left.1 \mathrm{H}, \mathrm{Ar}\right) \mathrm{ppm}$.<smiles>CCCOc1ccc2c(c1)NC(C)(C)C=C2C</smiles>

\section{7-[(tert-Butyldimethylsilyl)oxy]-1,2-dihydro-2,2,4-trimethylquinoline (287)}

Anhydrous ytterbium(III) triflate $(4.2 \mathrm{~g}, 6.8 \mathrm{mmol}, 6.5 \mathrm{~mol} \%$, freshly dried in vacuo at $130{ }^{\circ} \mathrm{C}$ for $4 \mathrm{~h}$ ) was added in one portion to a solution of compound 286-TBDMS (23.3 g, $0.1045 \mathrm{~mol})$ in dry acetone (300 mL). The reaction mixture was stirred at r.t. for $16 \mathrm{~h}$. Acetone was evaporated in vacuo, the residue was dissolved in AcOEt, washed with sat. aq. $\mathrm{NaHCO}_{3}$ (twice), water, brine and dried over $\mathrm{MgSO}_{4}$. After evaporation of solvents, the oily residue was dried in vacuo (0.5 Torr) to a constant weight. Purification by column chromatography (gradient elution with $n$-hexane to $n$-hexane/ $\mathrm{Et}_{2} \mathrm{O}=10 / 1$ ) afforded compound $287\left(R_{\mathrm{f}}=0.86, n\right.$-hexane/Et $\left.2 \mathrm{O}=8 / 1\right)$ as a clear oil $(19.85 \mathrm{~g}, 63 \%$ yield). ${ }^{1} \mathrm{H}$ NMR (400 MHz, $\mathrm{CDCl}_{3}$ ): $\delta=0.20$ (s, $6 \mathrm{H}, 2 \times \mathrm{Me}$ ), 0.99 (s, $9 \mathrm{H}, t \mathrm{Bu}$ ), 1.27 (s, $6 \mathrm{H}, 2 \times \mathrm{Me}$ ), 1.97 (d, $J=1.2 \mathrm{~Hz}, 3 \mathrm{H}, \mathrm{Me}$ ), 3.63 (br. s, $1 \mathrm{H}, \mathrm{NH}$ ), 5.20 (q, $J=1.2 \mathrm{~Hz}, 1$ H), 5.99 (d, $J=2.4,1 \mathrm{H}, \mathrm{Ar}$ ) , 6.15 (dd, $J=8.2$ and 2.4 Hz, $1 \mathrm{H}, \mathrm{Ar}$ ), 6.92 (d, $J=8.2 \mathrm{~Hz}$, $1 \mathrm{H}, \mathrm{Ar}) \mathrm{ppm} .{ }^{13} \mathrm{C}$ NMR $\left(125.7 \mathrm{MHz}, \mathrm{CDCl}_{3}\right): \delta=-4.4,18.2,18.6,25.7,30.9,51.9$, 
104.7, 108.9, 115.9, 124.5, 126.1, 128.3, 144.5, 156.1 ppm. HRMS (ESI, $\mathrm{C}_{18} \mathrm{H}_{29} \mathrm{NOSi}$ ): 302.1940 (found $[\mathrm{M}-\mathrm{H}]^{-}$), 302.1946 (calc.); HRMS (ESI, $\mathrm{C}_{18} \mathrm{H}_{29} \mathrm{NOSi}$ ): 304.2096 (found $\left.[\mathrm{M}+\mathrm{H}]^{+}\right), 304.2091$ (calc.).<smiles>CCOc1ccc2c(c1)N(CCCC(C)=O)C(C)(C)C=C2C</smiles>

1-[3-(Ethoxycarbonyl)propyl]-7-[(tert-butyldimethylsilyl)oxy]-1,2-dihydro-2,2,4-trimethylquinoline (288-TBDMS)

DIEA (11.8 g, $91.7 \mathrm{mmol}$ ) was added to a mixture of compound 288 (13.9 g, 45.87 mmol) and ethyl 3-iodobutyrate (13.3 g, 55.05 mmol) in a screw-cup bottle, and the reaction mixture was stirred with heating $\left(110^{\circ} \mathrm{C}\right)$ for 2 days. After cooling, the reaction mixture was diluted with $\mathrm{Et}_{2} \mathrm{O}$, passed through a plug of $\mathrm{SiO}_{2}$ (eluting with $\mathrm{Et}_{2} \mathrm{O}$ ), and the filtrate evaporated in vacuo. The residue was dissolved in $n$-hexane/ $\mathrm{Et}_{2} \mathrm{O}$ (3/1) mixture, washed with water, brine and dried over $\mathrm{MgSO}_{4}$. The product 288-TBDMS was isolated by a short path column chromatography ( $n$-hexane $\rightarrow n$-hexane/Et $2 \mathrm{O} 10 / 1 ; R_{\mathrm{f}}=0.59$ in $n$ hexane $\left./ \mathrm{Et}_{2} \mathrm{O}=10 / 1\right)$; yield $18.75 \mathrm{~g}$ (98\% yield) of a clear oil. ${ }^{1} \mathrm{H}$ NMR (400 MHz, $\mathrm{CDCl}_{3}$ ): $\delta=0.21$ (s, $6 \mathrm{H}, 2 \times \mathrm{Me}$ ), 0.99 (s, $\left.9 \mathrm{H}, t \mathrm{Bu}\right), 1.27$ (t, $\left.J=7.2 \mathrm{~Hz}, 3 \mathrm{H}, \mathrm{Et}\right), 1.28$ (s, $6 \mathrm{H}, 2 \times \mathrm{Me}$ ), 1.91 (m, 2 H, $\mathrm{CH}_{2}$ ), 1.94 (d, $\left.J=1.2 \mathrm{~Hz}, 3 \mathrm{H}, \mathrm{Me}\right), 2.38$ (m, $2 \mathrm{H}, \mathrm{CH}_{2}$ ), 3.21 (m, $2 \mathrm{H}, \mathrm{NCH}_{2}$ ), 4.16 (q, $\left.J=7.2 \mathrm{~Hz}, 2 \mathrm{H}, \mathrm{Et}\right), 5.11$ (q, $\left.J=1.2 \mathrm{~Hz}, 1 \mathrm{H}\right), 6.03$ (d, $J=2.4,1$ H, Ar), 6.12 (dd, $J=8.2$ and $2.4 \mathrm{~Hz}, 1 \mathrm{H}, \mathrm{Ar}$ ), 6.90 (d, $J=8.2 \mathrm{~Hz}, 1 \mathrm{H}, \mathrm{Ar}$ ) ppm. ${ }^{13} \mathrm{C} \mathrm{NMR}$ (125.7 MHz, $\left.\mathrm{CDCl}_{3}\right): \delta=-4.3,14.3,18.3,18.7,23.5,25.8,28.2,31.8,43.3,56.8,60.4$, 103.1, 107.1, 117.2, 124.4, 127.1, 127.6, 145.0, 156.4, 173.1 ppm.<smiles>CCOC(=O)CCCN1c2cc(O)ccc2C(C)=CC1(C)C</smiles>

1-[3-(Ethoxycarbonyl)propyl]-1,2-dihydro-7-hydroxy-2,2,4-trimethylquinoline (288H)

A solution of TBAF $3 \mathrm{H}_{2} \mathrm{O}(7.66 \mathrm{~g}, 24.31 \mathrm{mmol})$ in THF $(50 \mathrm{~mL})$ was added to a solution of ester 288-TBDMS (16.90 g, $40.53 \mathrm{mmol})$ in THF $(60 \mathrm{~mL})$ at $5{ }^{\circ} \mathrm{C}$. After $5 \mathrm{~min}$, the reaction mixture was diluted with $\mathrm{Et}_{2} \mathrm{O}(200 \mathrm{~mL})$, washed with water $(2 \times)$ and brine. The combined aqueous layers were extracted with ether, and the combined organic solutions 
were dried $\left(\mathrm{MgSO}_{4}\right)$. After evaporation of solvents, the residue was purified by column chromatography $\left(\mathrm{Et}_{2} \mathrm{O} / n\right.$-hexane, $\left.2 / 1 \rightarrow 4 / 1\right)$. Phenol 288- $\mathrm{H}\left(R_{\mathrm{f}}=0.07\right.$ in $n$-hexane/Et ${ }_{2} \mathrm{O}$, 1/5) was isolated in 99\% yield (12.15 g). ${ }^{1} \mathrm{H}$ NMR (400 MHz, $\mathrm{CDCl}_{3}$ ): $\delta=1.27$ (s, $6 \mathrm{H}$, 2×Me), 1.28 (t, $J=7.2 \mathrm{~Hz}, 3 \mathrm{H}, \mathrm{Et}), 1.92$ (m, $2 \mathrm{H}, \mathrm{CH}_{2}$ ), 1.93 (d, $J=1.2 \mathrm{~Hz}, 3 \mathrm{H}, \mathrm{Me}$ ), 2.39 (m, 2 H, $\mathrm{CH}_{2}$ ), 3.20 (m, 2 H, $\mathrm{NCH}_{2}$ ), 4.18 (q, $J=7.2 \mathrm{~Hz}, 2 \mathrm{H}, \mathrm{Et}$ ), 5.08 (q, $J=1.2$ $\mathrm{Hz}, 1 \mathrm{H}$ ), 6.13 (m, $2 \mathrm{H}, \mathrm{Ar}), 6.54$ (br. s, $1 \mathrm{H}, \mathrm{OH}), 6.90$ (d, $J=8.6 \mathrm{~Hz}, 1 \mathrm{H}, \mathrm{Ar}$ ) ppm. ${ }^{13} \mathrm{C}$ NMR (100.7 MHz, $\left.\mathrm{CDCl}_{3}\right): \delta=14.2,18.7,23.4,28.3,31.6,43.4,56.8,60.8,98.3,102.4$, 116.2, 124.8, 126.5, 127.6, 145.3, 156.9, 174.0 ppm.<smiles>CCOC(=O)CCCN1c2cc(O)c(C=O)cc2C(C)=CC1(C)C</smiles>

\section{1-[3-(Ethoxycarbonyl)propyl]-1,2-dihydro-6-formyl-7-hydroxy-2,2,4-} trimethylquinoline (289)

$\mathrm{POCl}_{3}(3.90 \mathrm{~g}, 25.5 \mathrm{mmol})$ was added to $\mathrm{DMF}(30 \mathrm{~mL})$ at $5^{\circ} \mathrm{C}$, and the mixture was allowed to warm to room temperature. After stirring for $15 \mathrm{~min}$ at room temperature, it was cooled down $\left(5^{\circ} \mathrm{C}\right.$ ), and a solution of phenol 288-Et (5.15 g, $17.0 \mathrm{mmol}$ ) in DMF (15 $\mathrm{mL}$ ) was added slowly. The cooling bath was removed, and the reaction mixture was allowed to warm up to room temperature, stirred for $0.5 \mathrm{~h}$, and finally heated at $50{ }^{\circ} \mathrm{C}$ for $15 \mathrm{~min}$. The TLC control of this reaction is difficult, because the product was found to have the same $R_{\mathrm{f}}$ value, as the starting material (in most solvents). After cooling, the reaction was "quenched" by adding $1 \mathrm{~mL}$ of sat. aq. $\mathrm{NaHCO}_{3}$, and the product 289 was extracted with dichloromethane. The organic layer was dried $\left(\mathrm{MgSO}_{4}\right)$, and, after evaporation of solvents, the residue was purified by column chromatography (gradient elution with hexane/ether mixture, $1 / 1$ to $1 / 4)$. Yield $3.59 \mathrm{~g}$ (64\%). ${ }^{1} \mathrm{H}$ NMR (400 $\left.\mathrm{MHz}, \mathrm{CDCl}_{3}\right)$ : $\delta=1.29(\mathrm{t}, J=7.2 \mathrm{~Hz}, 3 \mathrm{H}, \mathrm{Et}), 1.31$ (s, $6 \mathrm{H}, 2 \times \mathrm{Me}), 1.93\left(\mathrm{~m}, 2 \mathrm{H}, \mathrm{CH}_{2}\right), 1.96$ (d, $J=1.2$ $\mathrm{Hz}, 3 \mathrm{H}, \mathrm{Me}$ ), 2.40 (m, $2 \mathrm{H}, \mathrm{CH}_{2}$ ), 3.33 (m, $2 \mathrm{H}, \mathrm{NCH}_{2}$ ), 4.18 (q, J = 7.2 Hz, $2 \mathrm{H}, \mathrm{Et}$ ), 5.19 (q, $J=1.2 \mathrm{~Hz}, 1 \mathrm{H}), 5.98$ (s, $1 \mathrm{H}_{\mathrm{ar}}$ ), 7.03 (s, $\left.1 \mathrm{H}, \mathrm{Ar}\right), 9.48$ (s, $\left.1 \mathrm{H}\right), 11.76$ (s, $\left.1 \mathrm{H}\right)$ ppm. ${ }^{13} \mathrm{C}$ NMR (100.7 MHz, $\left.\mathrm{CDCl}_{3}\right): \delta=14.2(+)$, 18.6(+), 22.8(-), 29.3(+), 31.4(-), 44.0(-), 58.3(q), 60.7(-), 96.4(+), 110.9(-), 115.9(-), 126.1(-), 127.4(+), 128.1(+), 151.1(-), 164.7(-), 172.7(-), 191.9(+) ppm. HRMS (ESI, $\mathrm{C}_{19} \mathrm{H}_{25} \mathrm{NO}_{4}$ ): 330.1715 (found $[\mathrm{M}-\mathrm{H}]^{-}$), 330.1711 (calc.); HRMS (ESI, $\mathrm{C}_{19} \mathrm{H}_{25} \mathrm{NO}_{4}$ ): 354.1671 (found $[\mathrm{M}+\mathrm{Na}]^{+}$), 354.1676 (calc.); 332.1859 (found $[\mathrm{M}+\mathrm{H}]^{+}$), 332.1856 (calc.). 
<smiles>CCOC(=O)CCCN1c2cc3oc(=O)ccc3cc2C(C)=CC1(C)C</smiles>

Ethyl

4-(6,8,8-trimethyl-2-oxo-1,2,8,9-tetrahydropyrano[3,2-g]quinolin-9yl)butanoate (290)

Compound 289 and (ethoxycarbonylmethylene)triphenylphosphorane were dissolved in p-xylene, and refluxed for $3 \mathrm{~h}$ under argon. After cooling to r.t., the reaction mixture was filtered to remove triphenylphosphine oxide; afterwards the solvent was evaporated in vacuo. The residue was subjected to a column chromatography (100 g $\mathrm{SiO}_{2}$; hexane/EtOAc, $4: 1 \rightarrow 2: 1)$ to furnish $2.4 \mathrm{~g}(73 \%)$ of the titled product. ${ }^{1} \mathrm{H}$ NMR (300 $\mathrm{MHz}_{\mathrm{CDCl}}$ ): $\delta=1.28$ (t, $\left.J=7.1 \mathrm{~Hz}, 3 \mathrm{H}, \mathrm{Et}\right), 1.35$ (s, $6 \mathrm{H}, 2 \times \mathrm{Me}$ ), 1.80-1.95 (m, $2 \mathrm{H}$, $\mathrm{CH}_{2}$ ), 1.96 (d, $\left.J=1.4 \mathrm{~Hz}, 3 \mathrm{H}, \mathrm{Me}\right), 2.39$ (t, $J=6.9 \mathrm{~Hz}, 2 \mathrm{H}, \mathrm{CH}_{2}$ ), 3.25-3.35 (m, $2 \mathrm{H}$, $\mathrm{NCH}_{2}$ ), 4.17 (q, $J=7.1 \mathrm{~Hz}, 2 \mathrm{H}, \mathrm{CH}_{2}$ ), 5.26 (q, $J=1.4 \mathrm{~Hz}, 1 \mathrm{H}$ ), 6.01 (d, $J=9.3 \mathrm{~Hz}, 1 \mathrm{H}$, Ar), 6.34 (s, $1 \mathrm{H}, \mathrm{Ar}), 6.99$ (s, $1 \mathrm{H}, \mathrm{Ar}), 7.49$ (d, $J=9.3 \mathrm{~Hz}, 1 \mathrm{H}, \mathrm{Ar}) \mathrm{ppm} .{ }^{13} \mathrm{C} \mathrm{NMR}$ (125 MHz, $\left.\mathrm{CDCl}_{3}\right): \delta=14.2(+), 18.6(+), 22.6(-), 29.0\left(^{+}\right), 31.3(-), 43.8(-)$, 57.8(-), 60.6(-), 97.1(+), 108.3(-), 109.4(+), 120.1(-), 122.2(+), 126.3(-), 129.8(+), 143.8(+), 147.5(-), 156.6(-), 162.2(-), 172.8(-) ppm. HRMS (ESI): calc. for $\mathrm{C}_{21} \mathrm{H}_{24} \mathrm{NO}_{4}[\mathrm{M}+\mathrm{H}]^{+}$ 356.1856; found 356.1852.<smiles>CCOC(=O)CCCN1c2cc3oc(=O)ccc3cc2C(C=O)=CC1(C)C</smiles>

Ethyl 4-(6-formyl-8,8-dimethyl-2-oxo-1,2,8,9-tetrahydropyrano[3,2-g]quinolin-9yl)butanoate (291)

In a typical experiment, a $10 \mathrm{~mL}$ flask was charged with solution of compound $\mathbf{2 9 0}$ (300 mg, $0.845 \mathrm{mmol})$ in dioxane $(4 \mathrm{~mL})$ and finely powdered $\mathrm{SeO}_{2}$ (117 mg, $1.06 \mathrm{mmol})$. The resulted suspension refluxed for $3.5 \mathrm{~h}$, then, water $(0.5 \mathrm{~mL})$ was added, and the reaction mixture was allowed to cool to r.t. All volatile materials were evaporated in vacuo, the residue was dissolved in $\mathrm{CH}_{2} \mathrm{Cl}_{2}$ and washed with sat. aq. $\mathrm{NaHCO}_{3}$. Organic layer was dried with $\mathrm{Na}_{2} \mathrm{SO}_{4}$ and evaporated to give a crude product. The title compound was isolated as a yellow powder (235 $\mathrm{mg} ; 75 \%$ ) by means of column chromatography (30 $\mathrm{g} \mathrm{SiO}_{2}$, hexane/EtOAc, 1:1). ${ }^{1} \mathrm{H}$ NMR (300 MHz, $\mathrm{CDCl}_{3}$ ): $\delta=1.28$ (t, $J=7.1 \mathrm{~Hz}, 3$ 
H, Et), 1.50 (s, $6 \mathrm{H}, 2 \times \mathrm{Me}$ ), 1.85-1.97 (m, $2 \mathrm{H}, \mathrm{CH}_{2}$ ), 2.40 (t, $J=6.8 \mathrm{~Hz}, 2 \mathrm{H}, \mathrm{CH}_{2}$ ), 3.29-3.37 (m, 2 H, NCH $), 4.16$ (q, $J=7.1 \mathrm{~Hz}, 2 \mathrm{H}, \mathrm{Et}), 6.07$ (d, $J=9.3 \mathrm{~Hz}, 1 \mathrm{H}, \mathrm{Ar}$ ), 6.21 (s, $1 \mathrm{H}$ ), 6.44 (s, 1H, Ar), 7.56 (d, J = $9.3 \mathrm{~Hz}, 1 \mathrm{H}, \mathrm{Ar}$ ), 8.39 (s, $1 \mathrm{H}, \mathrm{Ar}$ ), 9.56 (s, 1 $\mathrm{H}, \mathrm{CHO})$ ppm. ${ }^{13} \mathrm{C}$ NMR (125 MHz, $\left.\mathrm{CDCl}_{3}\right): \delta=14.3(+), 22.9(-), 28.0(+), 31.3(-)$, 44.0(-), 58.1(-), 60.8(-), 98.3(+), 109.0(-), 110.5(+), 113.5(-), 125.4(+), 130.6(-), 143.9(+), 147.0(-), 151.9(+), 156.6(-), 161.6(-), 172.6(-), 191.6(+) ppm. HRMS (ESI): calc. for $\mathrm{C}_{21} \mathrm{H}_{23} \mathrm{NO}_{5}[\mathrm{M}+\mathrm{H}]^{+}$370.1649; found 370.1640 .<smiles>CCOC(=O)CCCN1c2cc3oc(=O)c(-c4ccccn4)cc3cc2C(C)=CC1(C)C</smiles>

Ethyl 4-(6,8,8-trimethyl-2-oxo-3-(pyridin-2-yl)-1,2,8,9-tetrahydropyrano[3,2g]quinolin-9-yl)butanoate (292)

To a suspension of 2-pyridylacetic acid (394 mg, $2.27 \mathrm{mmol}$ ) in DMF (5 mL) $\mathrm{NEt}_{3}$ $(400 \mu \mathrm{L})$ was added. The resulted mixture was stirred for $5 \mathrm{~min}$, then subsequently solution of aldehyde 289 (500 mg, $1.51 \mathrm{mmol})$ in $\mathrm{CH}_{2} \mathrm{Cl}_{2}(10 \mathrm{~mL})$, $\mathrm{EDC} \cdot \mathrm{HCl}$ (436 mg, $2.27 \mathrm{mmol}), \mathrm{NEt}_{3}(800 \mu \mathrm{L}), 4$-DMAP $(18.3 \mathrm{mg}, 0.15 \mathrm{mmol})$ were added. The reaction mixture was stirred overnight at r.t. Afterwards, water $(15 \mathrm{~mL})$ was added, organic phase was separated, and the aq. phase was extracted with $\mathrm{CH}_{2} \mathrm{Cl}_{2}$. The combined organic extracts were dried with $\mathrm{Na}_{2} \mathrm{SO}_{4}$ and evaporated. After a column chromatography (100 g $\mathrm{SiO}_{2}, n$-hexane/EtOAc, 2:1), the titled product was isolated in 35\% yield (227 $\mathrm{mg}$ ) as an orange solid. ${ }^{1} \mathrm{H}$ NMR (400 MHz, $\mathrm{CDCl}_{3}$ ): $\delta=1.29$ (t, $J=7.1 \mathrm{~Hz}, 3 \mathrm{H}, \mathrm{Me}$ ), 1.38 (s, $6 \mathrm{H}$, 2×Me), 1.89-1.97 (m, $2 \mathrm{H}, \mathrm{CH}_{2}$, overlapped), 1.97 (d, $J=1.3 \mathrm{~Hz}, 3 \mathrm{H}, \mathrm{Me}$ ), 2.40 (t, $J=6.9 \mathrm{~Hz}, 2 \mathrm{H}, \mathrm{CH}_{2}$ ), 3.29-3.37 (m, $2 \mathrm{H}, \mathrm{NCH}_{2}$ ), 4.18 (q, $J=7.1 \mathrm{~Hz}, 2 \mathrm{H}, \mathrm{CH}_{2}$ ), 5.28 (q, $J=1.3 \mathrm{~Hz}, 1 \mathrm{H}$ ), 6.37 (s, $1 \mathrm{H}, \mathrm{Ar}$ ), 7.16 (s, $1 \mathrm{H}, \mathrm{Ar}$ ), 7.17 (ddd, $J=7.8,4.8$ and $1.0 \mathrm{~Hz}$, $1 \mathrm{H}$, Ar, overlapped), 7.70 (ddd, $J=8.1,7.8$ and $1.9 \mathrm{~Hz}, 1 \mathrm{H}, \mathrm{Ar}$ ), 8.40 (dt, $J=8.1$ and $1.0 \mathrm{~Hz}, 1 \mathrm{H}, \mathrm{Ar}$ ), 8.60 (ddd, $J=4.8,1.9$ and $1.0 \mathrm{~Hz}, 1 \mathrm{H}, \mathrm{Ar}$ ), 8.64 (s, $1 \mathrm{H}, \mathrm{Ar}$ ) ppm. ${ }^{13} \mathrm{C}$ NMR (100.7 MHz, $\left.\mathrm{CDCl}_{3}\right): \delta=14.2(+), 18.7(+), 22.7(-), 29.2(+), 31.4(+), 44.0(-)$, 58.1(-), 60.7(-), 96.3(+), 109.1(-), 117.8(-), 120.4(-), 122.1(+), 123.2(+), 126.2(-), 129.6(+), 136.4(+), 142.9(+), 148.0(-), 149.0(+), 152.5(-), 156.7(-), 161.3(-), 172.7(-) ppm. HRMS (ESI): calc. for $\mathrm{C}_{26} \mathrm{H}_{28} \mathrm{~N}_{2} \mathrm{O}_{4}[\mathrm{M}+\mathrm{H}]^{+}$433.2122; found 433.2123. 
<smiles>CCOC(=O)CCCn1c(-c2cnccn2)cc2cc3c(cc21)N(C)C(C)(C)C=C3C</smiles>

Ethyl

4-(6,8,8-trimethyl-2-oxo-3-(pyrazin-2-yl)-1,2,8,9-tetrahydropyrano[3,2g]quinolin-9-yl)butanoate (293)

To a solution of 289 (166 mg, 0,5 mmol) in $\mathrm{CH}_{2} \mathrm{Cl}_{2}$ (3 mL) 2-(pyrazin-2-yl)acetic acid (69 mg, $0.5 \mathrm{mmol}$ ), $\mathrm{NEt}_{3}$ (106 mg, $1.05 \mathrm{mmol}$ ), DCC (103 mg, $0.5 \mathrm{mmol}$ ) and DMAP (6 mg, 10 mol\%) were added. The resulting mixture was allowed to stir overnight. The precipitated urea was filtered; the filtrate was evaporated under reduced pressure. The residue was subjected to a column chromatography (25 g of $\mathrm{SiO}_{2}, \mathrm{CH}_{2} \mathrm{Cl}_{2} / \mathrm{MeOH}, 30: 1$ ) to furnish $100 \mathrm{mg}$ (46\%) of a title product as an orange powder. ${ }^{1} \mathrm{H}$ NMR (300 MHz, $\mathrm{CDCl}_{3}$ ): $\delta=1.29$ (t, $J=7.1 \mathrm{~Hz}, 3 \mathrm{H}, \mathrm{Me}$ ), 1.39 (s, $6 \mathrm{H}, 2 \times \mathrm{Me}$ ), 1.89-1.97 (m, $2 \mathrm{H}, \mathrm{CH}_{2}$ ), 1.98 (d, $J=1.4 \mathrm{~Hz}, 3 \mathrm{H}, \mathrm{Me}$ ), 2.41 (t, $J=6.9 \mathrm{~Hz}, 2 \mathrm{H}, \mathrm{CH}_{2}$ ), 3.30-3.39 (m, $2 \mathrm{H}, \mathrm{CH}_{2}$ ), 4.18 (q, $J=7.1 \mathrm{~Hz}, 2 \mathrm{H}, \mathrm{CH}_{2}$ ), 5.30 (q, $J=1.4 \mathrm{~Hz}, 1 \mathrm{H}$ ), 6.39 (s, $1 \mathrm{H}, \mathrm{Ar}$ ), 7.15 (s, $1 \mathrm{H}$, Ar), 8.45 (d, $J=2.5 \mathrm{~Hz}, 1 \mathrm{H}, \mathrm{Ar}$ ), 8.54 (dd, $J=2.5$ and $1.5 \mathrm{~Hz}, 1 \mathrm{H}, \mathrm{Ar}$ ), 8.64 (s, $1 \mathrm{H}$, Ar), 9.66 (d, $J=1.5 \mathrm{~Hz}, 1 \mathrm{H}, \mathrm{Ar}) \mathrm{ppm} .{ }^{13} \mathrm{C} \mathrm{NMR}\left(125.7 \mathrm{MHz}, \mathrm{CDCl}_{3}\right): \delta=14.2(+)$, 18.6(+), 22.5(-), 29.2(+), 19.6(q), 31.3(-), 44.1(-), 58.3(-), 60.7(-), 96.3(+), 109.1(-), $114.9(-), 120.6(-), 123.4(+), 126.1(-), 129.9(+), 142.3\left(^{+}\right), 143.6(+), 144.0\left(^{+}\right), 144.4\left(^{+}\right)$, 148.8(-), 157.3(-), 160.8(-), $172.8(-)$ ppm. HRMS (ESI): calc. for $\mathrm{C}_{25} \mathrm{H}_{27} \mathrm{~N}_{3} \mathrm{O}_{4}[\mathrm{M}+\mathrm{H}]^{+}$ 456.1894; found 456.1878. UV-Vis spectral data in $\mathrm{CH}_{2} \mathrm{Cl}_{2}: \lambda_{\text {abs,max }}=451 \mathrm{~nm} ; \varepsilon=39200$ $\mathrm{M}^{-1} \mathrm{~cm}^{-1}, \lambda_{\text {em,max }}=506 \mathrm{~nm}, \Phi_{\mathrm{fl}}=0.74$ (standard: Lucifer Yellow, $\Phi_{\mathrm{fl}}=0.21$ in $\mathrm{H}_{2} \mathrm{O}$ ). UVVis spectral data in MeOH: $\lambda_{\mathrm{abs}, \max }=451 \mathrm{~nm} ; \varepsilon=39600 \mathrm{M}^{-1} \mathrm{~cm}^{-1}, \lambda_{\text {em,max }}=535 \mathrm{~nm}, \Phi_{\mathrm{fl}}=$ 0.08 (standard: Lucifer Yellow, $\Phi_{\mathrm{fl}}=0.21$ in $\mathrm{H}_{2} \mathrm{O}$ ).<smiles>CCOC(=O)CCCN1c2cc3oc(=O)c(-c4ccccn4)cc3cc2C(C=O)=CC1(C)C</smiles>

Ethyl 4-(6-formyl-8,8-dimethyl-2-oxo-3-(pyridin-2-yl)-1,2,8,9-tetrahydropyrano[3,2g]quinolin-9-yl)butanoate (294)

From compound 292: A round-bottomed flask was charged with solution of compound 292 (208 $\mathrm{mg}, 0.48 \mathrm{mmol})$ in dioxane $(5 \mathrm{~mL})$ and finely powdered $\mathrm{SeO}_{2}$ (67 $\mathrm{mg}$, 
$0.60 \mathrm{mmol})$. The resulted suspension refluxed for $3.5 \mathrm{~h}$, then water $(1 \mathrm{~mL})$ was added, and the reaction mixture was allowed to cool to r.t. All volatile materials were evaporated; the residue was dissolved in $\mathrm{CH}_{2} \mathrm{Cl}_{2}$, washed with saturated aq. $\mathrm{NaHCO}_{3}$, dried, and evaporated in vacuo. The title compound was isolated as a yellow solid (183 mg, 85\%) by means of column chromatography (40 g SiO $, \mathrm{CH}_{2} \mathrm{Cl}_{2} / \mathrm{Et}_{2} \mathrm{O}, 10: 1$ ). ${ }^{1} \mathrm{H}$ NMR (300 MHz, $\mathrm{CDCl}_{3}$ ): $\delta=1.29$ (t, $\left.J=7.1 \mathrm{~Hz}, 3 \mathrm{H}, \mathrm{Et}\right), 1.52$ (s, $6 \mathrm{H}, 2 \times \mathrm{Me}$ ), 1.881.99 (m, 2 H, $\mathrm{CH}_{2}$ ), 2.42 (t, $J=6.8 \mathrm{~Hz}, 2 \mathrm{H}, \mathrm{CH}_{2}$ ), 3.32-3.40 (m, $2 \mathrm{H}, \mathrm{NCH}_{2}$ ), 4.18 (q, $J=7.1 \mathrm{~Hz}, 2 \mathrm{H}, \mathrm{Et}$ ), 6.22 (s, $1 \mathrm{H}$ ), 6.47 (s, $1 \mathrm{H}, \mathrm{Ar}$ ), 7.22 (ddd, $J=7.5,4.8$ and $1.0 \mathrm{~Hz}$, $1 \mathrm{H}, \mathrm{Ar}$ ), 7.74 (ddd, $J=8.1,7.5$ and $1.9 \mathrm{~Hz}, 1 \mathrm{H}, \mathrm{Ar}$ ), 8.37 (dt, $J=8.1$ and $1.0 \mathrm{~Hz}, 1 \mathrm{H}$, Ar), 8.58 (s, 1 H, Ar), 8.63 (ddd, $J=4.8,1.9$ and $1.0 \mathrm{~Hz}, 1 \mathrm{H}, \mathrm{Ar}$ ), 8.71 (s, $1 \mathrm{H}, \mathrm{Ar}$ ) 9.58 (s, $1 \mathrm{H}, \mathrm{CHO}) \mathrm{ppm} .{ }^{13} \mathrm{C}$ NMR (125 MHz, $\left.\mathrm{CDCl}_{3}\right): \delta=14.3(+)$, 22.9(-), 28.2(+), 31.3(-), 44.2(-), 58.4(-), 60.8(-), 97.5(+), 109.7(-), 113.9(-), 122.3(+), 123.4(+), 126.6(+), 130.5(-), 136.7(+), 143.5(+), 147.6(-), 148.7(+), 151.4(+), 151.9(-), 156.8(-), 160.8(-), 172.6(-), 191.3(+) ppm. HRMS (ESI): calc. for $\mathrm{C}_{26} \mathrm{H}_{26} \mathrm{NO}_{5}[\mathrm{M}+\mathrm{H}]^{+}$447.1914; found 447.1906.

From compound 297: In a screw-cap tube compound 297 (50 mg, $0.11 \mathrm{mmol}$ ), 2-(tributylstannyl)pyridine (43 mg, $0.12 \mathrm{mmol})$, and $\mathrm{Pd}\left(\mathrm{PPh}_{3}\right)_{4}(6.3 \mathrm{mg}, 5 \mathrm{~mol} \%)$ in dioxane ( $1 \mathrm{~mL}$ ) were placed under argon. The mixture was heated to $110^{\circ} \mathrm{C}$ and left overnight at this temperature with stirring. Afterwards, the reaction mixture was allowed to cool to r.t., diluted with $\mathrm{CH}_{2} \mathrm{Cl}_{2}$ (10 mL), and water (5 mL) was added. The organic layer was separated; the aqueous phase was extracted with $\mathrm{CH}_{2} \mathrm{Cl}_{2}(3 \times 10 \mathrm{~mL})$. The combined organic extracts were dried and concentrated in vacuo to give a crude product. Column chromatography (25 $\mathrm{g} \mathrm{SiO}_{2}$, hexane/EtOAc, 1:1) furnished the desired product as a yellow solid (22 mg, $45 \%$ ).<smiles></smiles>

Ethyl 4-(6-formyl-3-(isoquinolin-6-yl)-8,8-dimethyl-2-oxo-1,2,8,9-tetrahydropyrano[3,2-g]quinolin-9-yl)butanoate (295)

In a screw-cap tube compound 297 (50 mg, $0.11 \mathrm{mmol}$ ), boronic ester 301 (34 mg, $0.13 \mathrm{mmol}), \mathrm{Pd}\left(\mathrm{PPh}_{3}\right)_{4}(6.5 \mathrm{mg}, 5 \mathrm{~mol} \%)$ and $2 \mathrm{M} \mathrm{Na}_{2} \mathrm{CO}_{3}(224 \mu \mathrm{L}, 0.45 \mathrm{mmol})$ in toluene $(1 \mathrm{~mL})$ were placed under argon. The mixture was heated to $110{ }^{\circ} \mathrm{C}$ and left 
overnight at this temperature with stirring. Afterwards, the reaction mixture was allowed to cool to r.t., diluted with $\mathrm{CH}_{2} \mathrm{Cl}_{2}(10 \mathrm{~mL})$, passed through a plug of Celite (eluting with $\mathrm{CH}_{2} \mathrm{Cl}_{2}$ ), and the filtrate evaporated in vacuo. Column chromatography (30 g of $\mathrm{SiO}_{2}$, $\mathrm{CH}_{2} \mathrm{Cl}_{2} / \mathrm{MeOH}, 30: 1$ ) furnished the title product as a yellow solid (31 mg, 56\%). ${ }^{1} \mathrm{H} \mathrm{NMR}$ (400 MHz, $\left.\mathrm{CDCl}_{3}\right): \delta=1.31(\mathrm{t}, J=7.1 \mathrm{~Hz}, 3 \mathrm{H}, \mathrm{Et}), 1.55$ (s, $\left.6 \mathrm{H}, 2 \times \mathrm{Me}\right), 1.91-2.01$ (m, 2 $\left.\mathrm{H}, \mathrm{CH}_{2}\right), 2.44$ (m, $2 \mathrm{H}, \mathrm{CH}_{2}$ ), 3.36-3.43 (m, $2 \mathrm{H}, \mathrm{NCH}_{2}$ ), 4.21 (q, J= $7.1 \mathrm{~Hz}, 2 \mathrm{H}, \mathrm{Et}$ ), 6.26 (s, $1 \mathrm{H}), 6.53$ (s, $1 \mathrm{H}, \mathrm{Ar}), 7.68-7.71$ (m, $1 \mathrm{H}, \mathrm{Ar}), 7.93$ (dd, $J=8.6 \mathrm{~Hz}$ and $1.7 \mathrm{~Hz}, 1$ H, Ar), 7.93 (s, $1 \mathrm{H}, \mathrm{Ar}$ ), 8.01 (d, $J=8.6 \mathrm{~Hz}, 1 \mathrm{H}, \mathrm{Ar}$ ), 8.22 (br. s, $1 \mathrm{H}, \mathrm{Ar}$ ), 8.54 (d, $J=5.8 \mathrm{~Hz}, 1 \mathrm{H}, \mathrm{Ar}), 9.25$ (s, $1 \mathrm{H}, \mathrm{Ar}), 9.61$ (s, $1 \mathrm{H}, \mathrm{CHO}) \mathrm{ppm} .{ }^{13} \mathrm{C}$ NMR (100 MHz, $\left.\mathrm{CDCl}_{3}\right): \delta=14.3(+), 22.8(-), 28.1(+), 31.2(-)$, 44.1(-), 58.3(-), 60.8(-), 97.8(+), 109.7(), 113.9(-), 120.7(+), 120.9(-), 125.9(+), 127.4(+), 127.5(+), 130.5(-), 135.8(-), 137.4(-), 142.0(+), 143.4(+), 147.5(-), 152.0(+), 156.5(-), 161.0(-), 172.7(-), 191.8(+) ppm. HRMS (ESI): calc. for $\mathrm{C}_{30} \mathrm{H}_{28} \mathrm{~N}_{2} \mathrm{O}_{5}[\mathrm{M}+\mathrm{H}]^{+}$497.2071; found 497.2059.<smiles>CCOC(=O)CCCN1c2cc3oc(=O)c(Br)cc3cc2C(C=O)=CC1(C)C</smiles>

Ethyl 4-(3-bromo-6-formyl-8,8-dimethyl-2-oxo-1,2,8,9-tetrahydropyrano[3,2g]quinolin-9-yl)butanoate (297)

To a solution of compound 291 (1032 mg, $2.8 \mathrm{mmol})$ in AcOH (10 mL) bromine solution (537 mg, $3.36 \mathrm{mmol}$ in $5 \mathrm{~mL}$ of $\mathrm{AcOH}$ ) was added dropwise under stirring. After $1 \mathrm{~h}$ stirring, the reaction mixture was poured onto water; the resulting slurry was extracted with $\mathrm{CH}_{2} \mathrm{Cl}_{2}(3 \times 20 \mathrm{~mL})$. Combined organic extracts were dried with $\mathrm{Na}_{2} \mathrm{SO}_{4}$ and evaporated. The crude product was purified by column chromatography (100 g $\mathrm{SiO}_{2}$, hexane/EtOAc, 1:1) to yield $1240 \mathrm{mg}$ (99\%) of title compound as a yellow powder. ${ }^{1} \mathrm{H}$ NMR (300 MHz, $\left.\mathrm{CDCl}_{3}, \mathrm{ppm}\right): \delta=1.28$ (t, $J=7.1 \mathrm{~Hz}, 3 \mathrm{H}, \mathrm{Et}$ ), 1.51 (s, $6 \mathrm{H}, 2 \times \mathrm{Me}$ ), 1.85-1.95 (m, $2 \mathrm{H}, \mathrm{CH}_{2}$ ), 2.40 (t, $J=6.8 \mathrm{~Hz}, 2 \mathrm{H}, \mathrm{CH}_{2}$ ), 3.29-3.37 (m, $2 \mathrm{H}, \mathrm{NCH}_{2}$ ), 4.17 (q, J = 7.1 Hz, $2 \mathrm{H}, \mathrm{Et}), 6.23$ (s, $1 \mathrm{H}$ ), 6.44 (s, $1 \mathrm{H}, \mathrm{Ar}$ ), 7.92 (s, $1 \mathrm{H}, \mathrm{Ar}$ ), 8.38 (s, $1 \mathrm{H}$, $\mathrm{Ar}$ ), 9.56 (s, $1 \mathrm{H}, \mathrm{CHO}) \mathrm{ppm} .{ }^{13} \mathrm{C} \mathrm{NMR}\left(125.7 \mathrm{MHz}, \mathrm{CDCl}_{3}\right): \delta=14.3(+), 22.8(-)$, 28.1(+), 31.2(-), 44.1(-), 58.3(-), 60.8(-), 98.0(+), 104.4(-), 109.5(-), 113.9(-), 124.7(+), 130.3(-), 144.9(+), 147.2(-), 152.0(+), 155.9(-), 157.8(-), 172.5(-), 191.4(+) ppm. HRMS (ESI): calc. for $\mathrm{C}_{21} \mathrm{H}_{22} \mathrm{BrNO}_{5}[\mathrm{M}+\mathrm{H}]^{+}$448.0754; found 448.0741 . 
$\mathrm{N}_{\mathrm{N}}^{\mathrm{N}} \mathrm{CO}_{2} \mathrm{H}$

\section{2-(Pyrazin-2-yl)acetic acid (299)}

A $100 \mathrm{~mL}$ Schlenk flask was charged with a solution of $i \mathrm{Pr}_{2} \mathrm{NH}(1290 \mathrm{mg}, 12.8 \mathrm{mmol})$ in THF (15 mL), cooled to $-78{ }^{\circ} \mathrm{C}$, and $1.6 \mathrm{M}$ solution of BuLi in hexanes (8 mL, 12.8 mmol) was injected. After $30 \mathrm{~min}$ of stirring at this temperature pyrazine (1 g, 10.6 mmol) was added. The reaction mixture was allowed to stir for $1 \mathrm{~h}$ and then was quenched with an excess of solid $\mathrm{CO}_{2}$. After the mixture warmed to r.t., water was added until clear phases formed. A pH was adjusted to 3 with conc. $\mathrm{HCl}$ with stirring under icecooling. Further extraction with EtOAc $(8 \times 50 \mathrm{~mL})$ gave organic solution, which was dried with $\mathrm{Na}_{2} \mathrm{SO}_{4}$ and evaporated at r.t. to furnish $915 \mathrm{mg}$ (62\%) of orange powder. This crude product was used directly without further purification. ${ }^{1} \mathrm{H}$ NMR (400 MHz, $\mathrm{CD}_{3} \mathrm{OD}$ ): $\delta=3.89$ (s, $2 \mathrm{H}, \mathrm{CH}_{2}$ ), 8.49 (d, $J=2.64 \mathrm{~Hz}, 1 \mathrm{H}, \mathrm{Ar}$ ), 8.54 (dd, $J=2.64$ and $1.52 \mathrm{~Hz}, 1 \mathrm{H}, \mathrm{Ar}$ ), 8.62 (d, $J=1.52 \mathrm{~Hz}, 1 \mathrm{H}, \mathrm{Ar}$ ) ppm. HRMS (ESI): found 137.0354; calc. for $\mathrm{C}_{6} \mathrm{H}_{6} \mathrm{~N}_{2} \mathrm{O}_{2}[\mathrm{M}-\mathrm{H}]^{-} 137.0357$.<smiles>CCOC(=O)CCCN1c2cc3cc(-c4ccccn4)c(=O)oc3cc2C(CO)=CC1(C)C</smiles>

Ethyl 4-(6-(hydroxymethyl)-8,8-dimethyl-2-oxo-3-(pyridin-2-yl)-1,2,8,9-tetrahydro pyrano[3,2-g]quinolin-9-yl)butanoate (282)

To a cooled solution $\left(0{ }^{\circ} \mathrm{C}\right)$ of compound 294 (67 mg, $\left.0.15 \mathrm{mmol}\right)$ in the solvent mixture (THF/MeOH, 1:1, $5 \mathrm{~mL}$ ) powder of $\mathrm{CeCl}_{3}(37 \mathrm{mg}, 0.15 \mathrm{mmol}$ ) was added. The resulting mixture was stirred until $\mathrm{CeCl}_{3}$ dissolved, and $\mathrm{NaBH}_{4}(6 \mathrm{mg}, 0.15 \mathrm{mmol})$ was added in one portion. Bright green fluorescence appeared immediately, and after 5 min sat. aq. $\mathrm{NH}_{4} \mathrm{Cl}(5 \mathrm{~mL})$ and water $(5 \mathrm{~mL})$ were added. The reaction mixture was extracted with $\mathrm{CH}_{2} \mathrm{Cl}_{2}(4 \times 10 \mathrm{~mL})$, the combined organic extracts were dried with $\mathrm{Na}_{2} \mathrm{SO}_{4}$ and evaporated. The residue was purified by column chromatography (30 g of $\mathrm{SiO}_{2}, \mathrm{CH}_{2} \mathrm{Cl}_{2} / \mathrm{MeOH}$, 25:1) to furnish an orange solid (27 mg; 85\%). ${ }^{1} \mathrm{H}$ NMR (300 MHz, $\left.\mathrm{CDCl}_{3}\right): \delta=1.29$ (t, $J=7.1 \mathrm{~Hz}, 3 \mathrm{H}, \mathrm{Et}), 1.36$ (s, $6 \mathrm{H}, 2 \times \mathrm{Me}$ ), 1.87-1.99 (m, $\left.2 \mathrm{H}, \mathrm{CH}_{2}\right), 2.41$ (t, $J=6.9 \mathrm{~Hz}$, $2 \mathrm{H}, \mathrm{CH}_{2}$ ), 3.27-3.36 (m, $2 \mathrm{H}, \mathrm{NCH}_{2}$ ), 4.20 (q, $\left.J=7.1 \mathrm{~Hz}, 2 \mathrm{H}, \mathrm{Et}\right), 4.45$ (d, $J=1.2 \mathrm{~Hz}$, $2 \mathrm{H}, \mathrm{CH}_{2}$ ), 5.47 (t, $J=1.2 \mathrm{~Hz}, 1 \mathrm{H}$ ), 6.37 (s, $1 \mathrm{H}, \mathrm{Ar}$ ), 7.18 (ddd, $J=7.5,4.8$ and $1.0 \mathrm{~Hz}$, 
$1 \mathrm{H}, \mathrm{Ar}$ ), 7.25 (s, $1 \mathrm{H}, \mathrm{Ar}$ ), 7.71 (ddd, $J=8.1,7.5$ and $1.9 \mathrm{~Hz}, 1 \mathrm{H}, \mathrm{Ar}$ ), 8.37 (dt, $J=8.1$ and $1.0 \mathrm{~Hz}, 1 \mathrm{H}, \mathrm{Ar}$ ), 8.56 (s, $1 \mathrm{H}, \mathrm{Ar}$ ), 8.59 (ddd, $J=4.8,1.9$ and $1.0 \mathrm{~Hz}, 1 \mathrm{H}, \mathrm{Ar}$ ) ppm. ${ }^{13} \mathrm{C}$ NMR (125.7 MHz, $\left.\mathrm{CDCl}_{3}\right): \delta=14.4(+), 22.7(-)$, 29.1(+), 31.4(-), 44.2(-), 58.0(-), 60.7(-), 62.8(-), 96.7(+), 109.1(-), 117.6(-), 117.9(-), 122.1(+), 123.0(+), 123.3(+), 129.3(+), 129.5(-), 136.4(+), 142.9(+), 148.0(-), 148.8(+), 152.3(-), 156.6(-), 161.1(-), 172.6(-) ppm. HRMS (ESI): calc. for $\mathrm{C}_{26} \mathrm{H}_{28} \mathrm{NO}_{5}[\mathrm{M}+\mathrm{H}]^{+}$449.2071; found 449.2071. UV-Vis spectral data in MeOH: $\lambda_{\mathrm{abs}, \max }=431 \mathrm{~nm} ; \varepsilon=32100 \mathrm{M}^{-1} \mathrm{~cm}^{-1}, \lambda_{\mathrm{em}, \max }=498 \mathrm{~nm}$, $\Phi_{\mathrm{fl}}=0.67$ (standard: Coumarin 334, $\Phi_{\mathrm{fl}}=0.69$ in EtOH).<smiles></smiles>

Ethyl 4-(6-(hydroxymethyl)-3-(isoquinolin-6-yl)-8,8-dimethyl-2-oxo-1,2,8,9tetrahydropyrano[3,2-g]quinolin-9-yl)butanoate (284)

To a cooled solution $\left(0^{\circ} \mathrm{C}\right)$ of compound 295 (31 mg, $\left.0.06 \mathrm{mmol}\right)$ in the solvent mixture (THF/MeOH, 1:1, $5 \mathrm{~mL}$ ) powder of $\mathrm{CeCl}_{3}(15 \mathrm{mg}, 0.06 \mathrm{mmol})$ was added. The resulting mixture was stirred until $\mathrm{CeCl}_{3}$ dissolved, and $\mathrm{NaBH}_{4}(2.5 \mathrm{mg}, 0.06 \mathrm{mmol})$ was added in one portion. Bright green fluorescence appeared immediately, and after 5 min, sat. aq. $\mathrm{NH}_{4} \mathrm{Cl}(2 \mathrm{~mL})$ and water $(2 \mathrm{~mL})$ were added. The reaction mixture was extracted with $\mathrm{CH}_{2} \mathrm{Cl}_{2}(3 \times 15 \mathrm{~mL})$; combined organic extracts were dried with $\mathrm{Na}_{2} \mathrm{SO}_{4}$ and evaporated. The residue was purified by column chromatography (30 g of $\mathrm{SiO}_{2}, \mathrm{CH}_{2} \mathrm{Cl}_{2} / \mathrm{MeOH}, 25: 1$ ) to furnish an orange solid (14 mg, 45\%). ${ }^{1} \mathrm{H}$ NMR (400 MHz, $\mathrm{CDCl}_{3}$ ): $\delta=1.32$ (t, $J=7.1 \mathrm{~Hz}, 3 \mathrm{H}, \mathrm{Et}), 1.44(\mathrm{~s}, 6 \mathrm{H}, 2 \times \mathrm{Me}), 1.91-2.02\left(\mathrm{~m}, 2 \mathrm{H}, \mathrm{CH}_{2}\right), 2.44\left(\mathrm{~m}, 2 \mathrm{H}, \mathrm{CH}_{2}\right)$, 3.37 (m, $2 \mathrm{H}, \mathrm{NCH}_{2}$ ), 4.21 (q, $J=7.1 \mathrm{~Hz}, 2 \mathrm{H}, \mathrm{Et}$ ), 4.51 (s, $2 \mathrm{H}, \mathrm{CH}_{2}$ ), 5.58 (s, $1 \mathrm{H}$ ), 6.46 (s, 1 H, Ar), 7.31 (s, 1 H, Ar), 7.69 (d, J=5.8 Hz, 1 H, Ar), 7.85 (s, 1 H, Ar), 7.92 (dd, $J=8.6$ and $1.7 \mathrm{~Hz}, 1 \mathrm{H}, \mathrm{Ar}$ ), 7.99 (d, $J=8.6 \mathrm{~Hz}, 1 \mathrm{H}, \mathrm{Ar}$ ), 8.22 (br. s, $1 \mathrm{H}, \mathrm{Ar}$ ), 8.53 (d, $J=5.8 \mathrm{~Hz}, 1 \mathrm{H}, \mathrm{Ar}$ ), 9.24 (s, $1 \mathrm{H}, \mathrm{Ar}$ ) ppm. ${ }^{13} \mathrm{C}$ NMR (100 MHz, $\left.\mathrm{CDCl}_{3}\right): \delta=14.3,22.7$, 29.0, 31.3, 44.0, 58.0, 60.7, 63.0, 97.0, 109.2, 117.7, 119.9, 120.8, 122.6, 125.6, 127.4, 127.5, 127.8, 129.7, 130.1, 135.8, 137.7, 141.8, 143.2, 148.0, 152.0, 156.4, 161.2, 172.7 ppm. MS (ESI): $m / z$ (positive mode, rel. int., \%) = $499(100)[\mathrm{M}+\mathrm{H}]^{+}$. UV-Vis spectral data in MeOH: $\lambda_{\mathrm{abs}, \max }=431 \mathrm{~nm} ; \varepsilon=41100 \mathrm{M}^{-1} \mathrm{~cm}^{-1}, \lambda_{\mathrm{em}, \max }=511 \mathrm{~nm}, \Phi_{\mathrm{fl}}=0.72$ (standard: Coumarin 522, $\Phi_{\mathrm{fl}}=0.65$ in EtOH). 


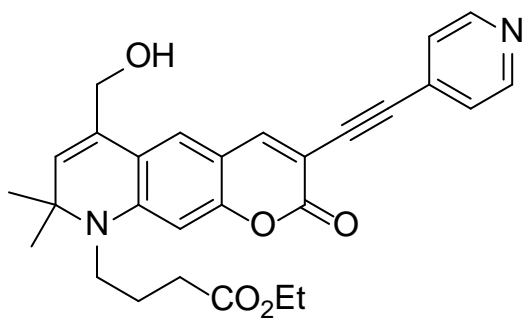

Ethyl 4-(6-(hydroxymethyl)-8,8-dimethyl-2-oxo-3-(pyridin-4-ylethynyl)-1,2,8,9tetrahydropyrano[3,2-g]quinolin-9-yl)butanoate (285)

To a cooled solution $\left(0^{\circ} \mathrm{C}\right)$ of compound 296 (80 $\left.\mathrm{mg}, 0.17 \mathrm{mmol}\right)$ in the solvent mixture (THF/MeOH, 2:1, $7 \mathrm{~mL}$ ) powder of $\mathrm{CeCl}_{3}(42 \mathrm{mg}, 0.17 \mathrm{mmol}$ ) was added. The resulting mixture was stirred until $\mathrm{CeCl}_{3}$ dissolved, and $\mathrm{NaBH}_{4}(6.5 \mathrm{mg}, 0.17 \mathrm{mmol})$ was added in one portion. Bright green fluorescence appeared immediately, and after $5 \mathrm{~min}$, an excess of acetone was added. The reaction mixture was evaporated, and the residue was taken in water $(\sim 15 \mathrm{~mL})$ and extracted with $\mathrm{CH}_{2} \mathrm{Cl}_{2}(3 \times 20 \mathrm{~mL})$. Combined organic extracts were dried with $\mathrm{Na}_{2} \mathrm{SO}_{4}$ and evaporated. Purification by column chromatography (30 g $\mathrm{SiO}_{2}$, $\mathrm{CH}_{2} \mathrm{Cl}_{2} / \mathrm{MeOH}, 25: 1$ ) furnished the title product as an orange solid (14 mg, $\left.45 \%\right) .{ }^{1} \mathrm{H}$ NMR (300 MHz, $\mathrm{CDCl}_{3}$ ): $\delta=1.29$ (t, $\left.J=7.1 \mathrm{~Hz}, 3 \mathrm{H}, \mathrm{Et}\right), 1.42$ (s, $6 \mathrm{H}, 2 \times \mathrm{Me}$ ), 1.85-1.97 (m, $2 \mathrm{H}, \mathrm{CH}_{2}$ ), 2.40 (m, $2 \mathrm{H}, \mathrm{CH}_{2}$ ), 3.34 (m, $2 \mathrm{H}, \mathrm{NCH}_{2}$ ), 4.18 (q, J = $7.1 \mathrm{~Hz}, 2$ H, Et), 4.45 (br. s, 2 H, CH ), 5.55 (s, 1 H), 6.38 (s, 1 H, Ar), 7.16 (s, 1 H, Ar), 7.35-7.39 (m, 2 H, Ar), 7.79 (s, $1 \mathrm{H}, \mathrm{Ar}$ ), 8.54-8.57 (m, $2 \mathrm{H}, \mathrm{Ar})$ ppm. ${ }^{13} \mathrm{C}$ NMR (125.7 MHz, $\left.\mathrm{CDCl}_{3}\right): \delta=14.3(+), 22.7(-), 29.2(+), 31.3(-), 44.2(-), 58.3(-), 60.8(-), 62.9(-), 89.4(-)$, 90.5(-), 97.2(+), 103.7(-), 108.4(-), 117.8(-), 122.3(+), 125.4(+), 129.3(-), 130.0(+), 131.2(-), 146.6(+), 148.5(-), 149.5(+), 156.4(-), 160.3(-), 172.5(-) ppm. HRMS (ESI): calc. for $\mathrm{C}_{28} \mathrm{H}_{28} \mathrm{~N}_{2} \mathrm{O}_{5}[\mathrm{M}+\mathrm{H}]^{+}$473.2071; found 473.2054. UV-Vis spectral data in MeOH: $\lambda_{\mathrm{abs}, \max }=457 \mathrm{~nm} ; \varepsilon=44200 \mathrm{M}^{-1} \mathrm{~cm}^{-1}, \lambda_{\mathrm{em}, \max }=511 \mathrm{~nm}, \Phi_{\mathrm{fl}}=0.62$ (standard: Coumarin $334, \Phi_{\mathrm{fl}}=0.69$ in $\left.\mathrm{EtOH}\right)$.<smiles>CC1(C)OB(c2ccc3cnccc3c2)OC1(C)C</smiles>

\section{6-(4,4,5,5-Tetramethyl-1,3,2-dioxaborolan-2-yl)isoquinoline (301)}

A dried $25 \mathrm{~mL}$ Schlenk flask was charged with a solution of 6-bromoisoquinoline (200 $\mathrm{mg}, 0.96 \mathrm{mmol})$ in THF $(7 \mathrm{~mL})$ and cooled down to $-78^{\circ} \mathrm{C}$. Afterwards, a solution of $t \mathrm{BuLi}(0.68 \mathrm{~mL}, 1.7 \mathrm{M}, 1.15 \mathrm{mmol})$ was injected. The resulting reaction mixture was stirred for $20 \mathrm{~min}$ at $-78{ }^{\circ} \mathrm{C}$, and $\mathrm{B}\left(\mathrm{OiPr}_{3}\right)(218 \mathrm{mg}, 1.15 \mathrm{mmol})$ was added. After over- 
night stirring at r.t., AcOH (86 mg, $1.44 \mathrm{mmol}$ ) and pinacol (226 mg, $1.92 \mathrm{mmol}$ ) were added. The reaction mixture was stirred for additional $2 \mathrm{~h}$ at r.t., and sat. aq. $\mathrm{NaCl}$ was added $(\sim 25 \mathrm{~mL})$. The resulted mixture was extracted with EtOAc $(3 \times 25 \mathrm{~mL})$. Combined organic extracts were dried with $\mathrm{Na}_{2} \mathrm{SO}_{4}$ and evaporated. The residue was subjected to column chromatography to afford $147 \mathrm{mg}(60 \%)$ of a clear oil. ${ }^{1} \mathrm{H}$ NMR (400 MHz, $\mathrm{CDCl}_{3}$ ): $\delta=1.40$ (s, $12 \mathrm{H}, 4 \times \mathrm{Me}$ ), 7.68 (d, $\left.J=5.7 \mathrm{~Hz}, 1 \mathrm{H}, \mathrm{Ar}\right), 7.93-7.99$ (m, $2 \mathrm{H}, \mathrm{Ar}$ ), 8.34 (s, $1 \mathrm{H}, \mathrm{Ar}$ ), 8.54 (d, $J=5.7 \mathrm{~Hz}, 1 \mathrm{H}, \mathrm{Ar}$ ), 9.27 (s, $1 \mathrm{H}, \mathrm{Ar}$ ) ppm. MS (ESI): m/z (positive mode, rel. int., \%) = $256(100)[\mathrm{M}+\mathrm{H}]^{+}$.<smiles></smiles>

Ethyl 4-(3-bromo-6-(hydroxymethyl)-8,8-dimethyl-2-oxo-1,2,8,9-tetrahydropyrano[3,2-g]quinolin-9-yl)butanoate (302)

To an ice cooled solution of compound 297 (100 mg; $0.22 \mathrm{mmol}$ ) in a solvent mixture (5 $\mathrm{mL}$, THF/MeOH, 1:1) powder of $\mathrm{CeCl}_{3}$ (54 mg; $0.22 \mathrm{mmol}$ ) was added under stirring. After its dissolution, $\mathrm{NaBH}_{4}$ (8.5 mg; $0.22 \mathrm{mmol}$ ) was added in one portion. The reaction mixture was stirred for $5 \mathrm{~min}$, acetone $(5 \mathrm{~mL})$ was added, and reaction mixture was allowed to warm up to r.t. All volatile materials were evaporated in vacuo, the residue was taken up in water $(10 \mathrm{~mL})$ and extracted with $\mathrm{CHCl}_{3}(3 \times 10 \mathrm{~mL})$. The combined organic liquids were dried and concentrated to give crude product. The title compound was purified by means of column chromatography (25 g of $\mathrm{SiO}_{2}, \mathrm{CH}_{2} \mathrm{Cl}_{2} / \mathrm{MeOH}, 25: 1$ ) as a yellow amorphous solid (96 mg; 95\%). ${ }^{1} \mathrm{H}$ NMR (400 $\mathrm{MHz}, \mathrm{CDCl}_{3}$ ): $\delta=1.30$ (t, $J=7.1 \mathrm{~Hz}, 3 \mathrm{H}, \mathrm{Et}), 1.42$ (s, $6 \mathrm{H}, 2 \times \mathrm{Me}$ ), 1.87-1.96 (m, $2 \mathrm{H}, \mathrm{CH}_{2}$ ), 2.41 (t, $J=6.9 \mathrm{~Hz}, 2$ H, $\mathrm{CH}_{2}$ ), 3.29-3.35 (m, 2 H, $\mathrm{NCH}_{2}$ ), 4.19 (q, J = 7.1 Hz, $2 \mathrm{H}, \mathrm{Et}$ ), 4.45 (br. s, $2 \mathrm{H}, \mathrm{CH}_{2}$ ), 5.55 (t, $J=1.2 \mathrm{~Hz}, 1 \mathrm{H}$ ), 7.1 (s, $1 \mathrm{H}, \mathrm{Ar}), 7.87$ (s, $1 \mathrm{H}, \mathrm{Ar}) \mathrm{ppm} .{ }^{13} \mathrm{C}$ NMR (100 MHz, $\left.\mathrm{CDCl}_{3}\right): \delta=14.2(+), 22.6(-), 29.0(+), 31.3(-), 44.0(-), 57.9(-), 60.7(-), 63.0(-), 97.2\left(^{+}\right)$, 103.4(-), 109.1(-), 117.8(-), 121.5(+), 129.5(-), 130.2(+), 144.7(+), 147.8(-), 155.9(-), 172.7(-) ppm. HRMS (ESI): calc. for $\mathrm{C}_{26} \mathrm{H}_{28} \mathrm{~N}_{2} \mathrm{O}_{5}[\mathrm{M}+\mathrm{H}]^{+}$449.2071; found 449.2071. 
<smiles>CCCOCC1=CC(C)(C)N(CCCOCC)c2cc3cc(Br)c(=O)oc3cc21</smiles>

Ethyl 4-(3-bromo-6-((di-tert-butoxyphosphoryloxy)methyl)-8,8-dimethyl-2-oxo1,2,8,9-tetrahydropyrano[3,2-g]quinolin-9-yl)butanoate (303)

To a stirred and preheated $\left(40{ }^{\circ} \mathrm{C}\right)$ solution of compound 302 (130 mg, $\left.0.29 \mathrm{mmol}\right)$ in $\mathrm{CH}_{2} \mathrm{Cl}_{2}(10 \mathrm{~mL})$ di-t-butyl $N, N$-diisopropylphosphoramidite (240 mg, $0.87 \mathrm{mmol}$ ) and $1 H$-tetrazole (65 mg, $0.93 \mathrm{mmol}$ ) were added in two equal portions at interval of $20 \mathrm{~min}$ under Ar. After further 20 min the reaction mixture was cooled with ice bath $\left(0^{\circ} \mathrm{C}\right)$ and solution of mCPBA (214 mg, $70 \%$ purity, $0.87 \mathrm{mmol}$ ) in $\mathrm{CH}_{2} \mathrm{Cl}_{2}$ was added. After stirring for additional $30 \mathrm{~min}$ aqueous solutions of $\mathrm{Na}_{2} \mathrm{SO}_{3}(4 \mathrm{~mL}, 10 \%)$ and $\mathrm{NaHCO}_{3}(5 \mathrm{~mL}$, saturated) were added, and the reaction mixture was allowed to warm up to r.t. The organic layer was separated and the aqueous phase was extracted with $\mathrm{CH}_{2} \mathrm{Cl}_{2}(3 \times 20$ $\mathrm{mL}$ ). The combined organic extracts were dried, the solvents were evaporated, and the titled compound was isolated by column chromatography (30 g of $\mathrm{SiO}_{2}, n$-hexane/EtOAc, 1:1) as a yellow amorphous solid (162 mg, 87\%). ${ }^{1} \mathrm{H}$ NMR (400 $\mathrm{MHz}, \mathrm{CDCl}_{3}$ ): $\delta=1.29$ (t, $J=7.1 \mathrm{~Hz}, 3 \mathrm{H}, \mathrm{Et}), 1.41$ (s, $6 \mathrm{H}, 2 \times \mathrm{Me}), 1.48$ (s, $18 \mathrm{H}, 2 \times t \mathrm{Bu}), 1.86-1.95$ (m, $2 \mathrm{H}$, $\mathrm{CH}_{2}$ ), 2.40 (t, $J=6.8 \mathrm{~Hz}, 2 \mathrm{H}, \mathrm{CH}_{2}$ ), 3.28-3.34 (m, 2H, $\mathrm{NCH}_{2}$ ), 4.18 (q, $J=7.1 \mathrm{~Hz}, 2 \mathrm{H}$, Et), $4.72\left(\mathrm{~d},{ }^{3} J_{\mathrm{HP}}=7.4 \mathrm{~Hz}, 2 \mathrm{H}, \mathrm{CH}_{2}\right), 5.61(\mathrm{~s}, 1 \mathrm{H}), 6.38$ (s, $\left.1 \mathrm{H}, \mathrm{Ar}\right), 7.13$ (s, $1 \mathrm{H}, \mathrm{Ar}$ ), 7.86 (s, $1 \mathrm{H}, \mathrm{Ar}) \mathrm{ppm} .{ }^{13} \mathrm{C} \mathrm{NMR}\left(100.7 \mathrm{MHz}, \mathrm{CDCl}_{3}\right): \delta=14.2(+)$, 22.6(+), 28.9(+), 29.9(+, d, $\left.{ }^{3} J_{\mathrm{CP}}=4.3 \mathrm{~Hz}\right), 31.3(-), 44.0(-), 58.0(-), 60.7(-), 66.0\left(-, \mathrm{d},{ }^{2} J_{\mathrm{CP}}=5.5 \mathrm{~Hz}\right), 82.7$ $\left(-, \quad\right.$ d, $\left.{ }^{2} J_{\mathrm{CP}}=7.4 \mathrm{~Hz}\right), 97.2(+), 103.5(-), 109.1(-), 117.4(-), 121.6(+), 126.1(-, \mathrm{d}$, $\left.{ }^{3} J_{\mathrm{CP}}=7.8 \mathrm{~Hz}\right), 132.0(+), 144.7(+), 147.6(-), 155.9(-), 158.1(-), 172.7(-)$ ppm. HRMS (ESI): calc. for $\mathrm{C}_{29} \mathrm{H}_{41} \mathrm{BrNO}_{8} \mathrm{P}[\mathrm{M}+\mathrm{H}]^{+}$642.1826; found 642.1818 .<smiles>CCCOCC1=CC(C)(C)N(CCCOCC)c2cc3cc(-c4ccccn4)c(=O)oc3cc21</smiles>

Ethyl 4-(6-((di-tert-butoxyphosphoryloxy)methyl)-8,8-dimethyl-2-oxo-3-(pyridin-2yl)-1,2,8,9-tetrahydropyrano[3,2-g]quinolin-9-yl)butanoate (304) 
From compound 282: To a stirred and preheated $\left(40^{\circ} \mathrm{C}\right)$ solution of compound 282 (70 mg, $0.16 \mathrm{mmol})$ in $\mathrm{CH}_{2} \mathrm{Cl}_{2}(10 \mathrm{~mL})$ di-t-butyl $N, N$-diisopropylphosphoramidite (130 mg, $0.47 \mathrm{mmol}$ ) and $1 \mathrm{H}$-tetrazole (35 mg, $0.5 \mathrm{mmol}$ ) were added in two equal portions at interval of 20 min under argon. After further 20 min the reaction mixture was cooled with ice bath $\left(0^{\circ} \mathrm{C}\right)$, and solution of $\mathrm{mCPBA}(115 \mathrm{mg}, 70 \%$ purity, $0.47 \mathrm{mmol})$ in $\mathrm{CH}_{2} \mathrm{Cl}_{2}$ was added. After stirring for additional 30 min aqueous solutions of $\mathrm{Na}_{2} \mathrm{SO}_{3}$ (2 $\mathrm{mL}, 10 \%)$ and $\mathrm{NaHCO}_{3}$ (2 mL, saturated) were added, and the reaction mixture was allowed to warm up to r.t. The organic layer was separated and the aqueous phase was extracted with $\mathrm{CH}_{2} \mathrm{Cl}_{2}(3 \times 10 \mathrm{~mL})$. The combined organic extracts were dried, the solvents were evaporated, and the titled compound was isolated by column chromatography (30 g of $\mathrm{SiO}_{2}, \mathrm{CH}_{2} \mathrm{Cl}_{2} / \mathrm{MeOH}, 25: 1$ ) as an orange amorphous solid (88 mg, 88\%). ${ }^{1} \mathrm{H}$ NMR (400 MHz, $\mathrm{CDCl}_{3}$ ): $\delta=1.31$ (t, $J=7.1 \mathrm{~Hz}, 3 \mathrm{H}, \mathrm{Et}$ ), 1.43 (s, $6 \mathrm{H}$, 2×Me), 1.50 (s, $18 \mathrm{H}, 2 \times t \mathrm{Bu}), 1.90-1.99\left(\mathrm{~m}, 2 \mathrm{H}, \mathrm{CH}_{2}\right), 2.42$ (t, $J=6.9 \mathrm{~Hz}, 2 \mathrm{H}, \mathrm{CH}_{2}$ ), 3.33-3.38 (m, $2 \mathrm{H}, \mathrm{NCH}_{2}$ ), 4.20 (q, J=7.1 Hz, $2 \mathrm{H}, \mathrm{Et}$ ), 4.75 (dd, ${ }^{3} J_{\mathrm{HP}}=7.3 \mathrm{~Hz}$, ${ }^{4} J_{\mathrm{HH}}=1.2 \mathrm{~Hz}, 2 \mathrm{H}, \mathrm{CH}_{2}$ ), $5.64(\mathrm{t}, J=1.2 \mathrm{~Hz}, 1 \mathrm{H}$ ), 6.42 (s, $1 \mathrm{H}, \mathrm{Ar}$ ), 7.19 (ddd, $J=7.5$, 4.8 and $1.0 \mathrm{~Hz}, 1 \mathrm{H}, \mathrm{Ar}$ ), 7.26 (s, $1 \mathrm{H}, \mathrm{Ar}$ ), 7.72 (ddd, $J=8.1,7.5$ and $1.9 \mathrm{~Hz}, 1 \mathrm{H}, \mathrm{Ar}$ ), 8.39 (dt, $J=8.1$ and $1.0 \mathrm{~Hz}, 1 \mathrm{H}, \mathrm{Ar}$ ), 8.62 (ddd, $J=4.8,1.9$ and $1.0 \mathrm{~Hz}, 1 \mathrm{H}, \mathrm{Ar}$ ), 8.65 (s, $1 \mathrm{H}, \mathrm{Ar}) \mathrm{ppm} .{ }^{13} \mathrm{C} \mathrm{NMR}\left(100.7 \mathrm{MHz}, \mathrm{CDCl}_{3}\right): \delta=14.3(+), 22.7(-)$, 28.9(+), $29.9\left(^{+}\right.$, d, $\left.{ }^{3} J_{\mathrm{CP}}=4.3 \mathrm{~Hz}\right), 31.3(-), 44.1(-), 58.0(-), 60.7(-), 65.8\left(-, \mathrm{d},{ }^{2} J_{\mathrm{CP}}=5.5 \mathrm{~Hz}\right), 82.6(-, \mathrm{d}$, $\left.{ }^{2} J_{\mathrm{CP}}=7.4 \mathrm{~Hz}\right), 96.7(+), 109.3(-), 117.4(-), 118.4(-), 122.2(+), 123.1(+), 123.2(+)$, 126.2(-, d, $\left.{ }^{3} J_{\mathrm{CP}}=7.8 \mathrm{~Hz}\right), 131.1(+), 136.4(+), 142.9(+), 147.9(-), 149.1(+), 152.4(-)$, 156.7(-), 161.2(-), 172.7(-) ppm. HRMS (ESI): calc. for $\mathrm{C}_{34} \mathrm{H}_{45} \mathrm{~N}_{2} \mathrm{O}_{8} \mathrm{P}[\mathrm{M}+\mathrm{H}]^{+}$ 641.2986; found 641.2986.

From compound 303: A $10 \mathrm{~mL}$ Schlenk flask was flushed with Ar and charged consequently with toluene $(0.5 \mathrm{~mL}), \mathrm{Pd}(\mathrm{OAc})_{2}\left(1 \mathrm{mg} ; 4.5 \cdot 10^{-3} \mathrm{mmol}\right)$, the solution of $\mathrm{P}(t-\mathrm{Bu})_{3}$ in dioxane $\left(0.395 \mathrm{M}, 23 \mu \mathrm{l}, 9 \cdot 10^{-3} \mathrm{mmol}\right.$ ), the solution of bromide 303 (48 $\mathrm{mg} ; 0.075$ $\mathrm{mmol}$ ) in toluene (1 mL) and 2-(tributylstannyl)pyridine (30 mg; $0.082 \mathrm{mmol})$. The reaction mixtire was stirred at $110{ }^{\circ} \mathrm{C}$ for $2 \mathrm{~h}$, cooled to r.t., and water (5 mL) and $\mathrm{CH}_{2} \mathrm{Cl}_{2}$ were added. The organic phase was separated; aqueous layer was extracted with $\mathrm{CH}_{2} \mathrm{Cl}_{2}$ $(3 \times 10 \mathrm{~mL})$. The combined organic extracts were dried with $\mathrm{Na}_{2} \mathrm{SO}_{4}$ and evaporated. A purification of the crude product by chromatography (30 g of $\mathrm{SiO}_{2}, \mathrm{CH}_{2} \mathrm{Cl}_{2} / \mathrm{MeOH}, 25: 1$ ) gave $27 \mathrm{mg}$ (56\%) of a red solid. 
<smiles>CCCOCC1=CC(C)(C)N(CCCOCC)c2cc3cc(C#Cc4ccncc4)c(=O)oc3cc21</smiles>

Ethyl 4-(6-((di-tert-butoxyphosphoryloxy)methyl)-8,8-dimethyl-2-oxo-3-(pyridin-4ylethynyl)-1,2,8,9-tetrahydropyrano[3,2-g]quinolin-9-yl)butanoate (305)

To a stirred and preheated $\left(40{ }^{\circ} \mathrm{C}\right)$ solution of compound 285 (20 mg, $\left.0.04 \mathrm{mmol}\right)$ in $\mathrm{CH}_{2} \mathrm{Cl}_{2}(4 \mathrm{~mL})$ di-t-butyl $N$, $N$-diisopropylphosphoramidite (16 mg, $0.06 \mathrm{mmol}$ ) and $1 \mathrm{H}$ tetrazole ( $5 \mathrm{mg}, 0.07 \mathrm{mmol}$ ) were added in two equal portions at interval of 20 min under argon. After further $20 \mathrm{~min}$ the reaction mixture was cooled down with ice bath $\left(0{ }^{\circ} \mathrm{C}\right)$, and solution of $m$ CPBA (15.5 mg, $70 \%$ purity, $0.06 \mathrm{mmol}$ ) in $\mathrm{CH}_{2} \mathrm{Cl}_{2}(2 \mathrm{~mL})$ was added. After stirring for additional $15 \mathrm{~min}$, aqueous solutions of $\mathrm{Na}_{2} \mathrm{SO}_{3}(2 \mathrm{~mL}, 10 \%)$ and $\mathrm{NaHCO}_{3}$ (2 $\mathrm{mL}$, saturated) were added, and the reaction mixture was allowed to warm up to r.t. The organic layer was separated, and the aqueous phase was extracted with $\mathrm{CH}_{2} \mathrm{Cl}_{2}$ $(2 \times 10 \mathrm{~mL})$. The combined organic extracts were dried, the solvents were evaporated, and the titled compound was isolated by column chromatography (25 g of $\mathrm{SiO}_{2}$, $\mathrm{CH}_{2} \mathrm{Cl}_{2} / \mathrm{MeOH}, 25: 1$ ) as an orange amorphous solid (23 mg; 82\%). ${ }^{1} \mathrm{H}$ NMR (400 MHz, $\mathrm{CDCl}_{3}$ ): $\delta=1.19$ (t, $J=7.1 \mathrm{~Hz}, 3 \mathrm{H}, \mathrm{Et}$ ), 1.42 (s, $6 \mathrm{H}, 2 \times \mathrm{Me}$ ), 1.48 (br. s, $18 \mathrm{H}, 2 \times t \mathrm{Bu}$ ), 1.87-1.95 (m, 2 H, CH$)_{2}$ ), 2.40 (t, $J=6.8 \mathrm{~Hz}, 2 \mathrm{H}, \mathrm{CH}_{2}$ ), 3.30-3.38 (m, $2 \mathrm{H}, \mathrm{NCH}_{2}$ ), 4.18 (q, $J=7.1 \mathrm{~Hz}, 2 \mathrm{H}, \mathrm{Et}), 4.75$ (d, ${ }^{3} J_{\mathrm{HP}}=7.0 \mathrm{~Hz}, 2 \mathrm{H}, \mathrm{CH}_{2}$ ), $5.61(\mathrm{~s}, 1 \mathrm{H}), 6.38$ (s, $1 \mathrm{H}, \mathrm{Ar}$ ), 7.18 (s, 1 H, Ar), 7.36-7.39 (m, 2 H, Ar), 7.79 (s, 1 H, Ar), 8.55-8.58 (m, 2 H, Ar) ppm. HRMS (ESI): calc. for $\mathrm{C}_{36} \mathrm{H}_{45} \mathrm{~N}_{2} \mathrm{O}_{8} \mathrm{P}[\mathrm{M}+\mathrm{H}]^{+}$665.2986; found 665.2984.<smiles>CCCOCC1=CC(C)(C)N(CCCC(=O)O)c2cc3oc(=O)c(-c4ccccn4)cc3cc21</smiles>

4-(6-((Di-tert-butoxyphosphoryloxy)methyl)-8,8-dimethyl-2-oxo-3-(pyridin-2-yl)1,2,8,9-tetrahydropyrano[3,2-g]quinolin-9-yl)butanoic acid (306)

To a solution of 304 (98 mg, $0.15 \mathrm{mmol}$ ) in the solvent mixture (20 mL, THF/water, 3:2) $1 \mathrm{M}$ solution of $\mathrm{NaOH}(0.6 \mathrm{~mL}, 0.6 \mathrm{mmol})$ was added. The reaction mixture was stirred overnight at r.t., then its acidity was adjusted to $\mathrm{pH} 4$ with sat. aq. $\mathrm{KHSO}_{4}$. The resulted solution was extracted with EtOAc $(5 \times 25 \mathrm{~mL})$, the combined organic extracts were dried 
and concentrated in vacuo. The titled compound was isolated by column chromatography (30 g of $\mathrm{SiO}_{2}, \mathrm{CH}_{2} \mathrm{Cl}_{2} / \mathrm{MeOH}, 15: 1$ ) as a red solid (60 mg; 65\%). ${ }^{1} \mathrm{H}$ NMR (400 MHz, $\mathrm{CDCl}_{3}$ ): $\delta=1.40$ (s, $\left.6 \mathrm{H}, 2 \times \mathrm{Me}\right), 1.50$ (s, $\left.18 \mathrm{H}, 2 \times t \mathrm{Bu}\right), 1.90-2.00\left(\mathrm{~m}, 2 \mathrm{H}, \mathrm{CH}_{2}\right), 2.47$ (t, $\left.J=6.8 \mathrm{~Hz}, 2 \mathrm{H}, \mathrm{CH}_{2}\right), 3.33-3.39\left(\mathrm{~m}, 2 \mathrm{H}, \mathrm{CH}_{2}\right), 4.78$ (dd, $J_{\mathrm{HP}}=7.5 \mathrm{~Hz}, J_{\mathrm{HH}}=1.1 \mathrm{~Hz}, 2$ H, $\mathrm{CH}_{2}$ ), 5.64 (d, $J=1.1 \mathrm{~Hz}, 1 \mathrm{H}$ ), 6.43 (s, $1 \mathrm{H}, \mathrm{Ar}$ ), 7.21 (ddd, $J=7.5,4.9$ and $1.1 \mathrm{~Hz}, 1$ H, Ar), 7.73 (ddd, $J=8.0,7.5$ and $1.9 \mathrm{~Hz}, 1 \mathrm{H}, \mathrm{Ar}$ ), 8.35 (dt, $J=8.0$ and $1.1 \mathrm{~Hz}, 1 \mathrm{H}, \mathrm{Ar}$ ), 8.59 (s, $1 \mathrm{H}, \mathrm{Ar}$ ), 8.64 (ddd, $J=4.9,1.9$ and $1.1 \mathrm{~Hz}, 1 \mathrm{H}, \mathrm{Ar}$ ) ppm. ${ }^{13} \mathrm{C}$ NMR (100 MHz, $\left.\mathrm{CDCl}_{3}\right): \delta=14.1(+), 22.7(-), 28.8(+), 29.9\left(+, \mathrm{d}, J_{\mathrm{CP}}=4.3 \mathrm{~Hz}\right), 31.1(-), 43.9(-), 58.0(-)$, 66.0(-, d, $\left.J_{\mathrm{CP}}=5.5 \mathrm{~Hz}\right), 83.1\left(-\right.$, d, $\left.J_{\mathrm{CP}}=7.6 \mathrm{~Hz}\right), 96.9(+), 109.3(-), 117.5(-), 118.2(-)$, 122.3(+), 123.1(+), 123.4(+), 126.2(-, d, $\left.J_{\mathrm{CP}}=7.7 \mathrm{~Hz}\right), 131.4(+), 136.6(+), 143.1(+)$, 148.0(-), 148.9(+), 152.4(-), 156.7(-), 161.2(-), 176.7(-) ppm. HRMS (ESI): calc. for $\mathrm{C}_{32} \mathrm{H}_{41} \mathrm{~N}_{2} \mathrm{O}_{8} \mathrm{P}[\mathrm{M}-\mathrm{H}]^{-}$611.2528; found 611.2517.<smiles>CC1(C)C=C(COP(O)O)c2cc3cc(-c4ccccn4)c(=O)oc3cc2N1CCCC(=O)O</smiles>

4-(8,8-Dimethyl-2-oxo-6-(phosphonooxymethyl)-3-(pyridin-2-yl)-1,2,8,9-tetrahydropyrano[3,2-g]quinolin-9-yl)butanoic acid (308)

To a solution of 307 (60 mg, $0.10 \mathrm{mmol})$ in $\mathrm{CH}_{2} \mathrm{Cl}_{2}(5 \mathrm{~mL})$ trifluoroacetic acid $(0.3 \mathrm{~mL})$ was added. The resulted mixture was stirred for $30 \mathrm{~min}$. Then all volatile substances were evaporated in vacuo, and the residue was subjected to a column chromatography (20 g of $\mathrm{SiO}_{2}, \mathrm{MeCN} /$ water, $2: 1+0.1 \% \mathrm{NEt}_{3}$ ) to furnish $56 \mathrm{mg}$ of red amorphous solid (as 308.3NEt 3 , 78\%). ${ }^{1} \mathrm{H}$ NMR (400 MHz, $\left.\mathrm{CD}_{3} \mathrm{OD}\right): \delta=1.29$ (t, $J=7.3 \mathrm{~Hz}, 27 \mathrm{H}, 9 \times \mathrm{Et}$ ), 1.41 (s, $6 \mathrm{H}, 2 \times \mathrm{Me}$ ), 1.84-1.93 (m, $2 \mathrm{H}, \mathrm{CH}_{2}$ ), 2.44 (t, $J=6.7 \mathrm{~Hz}, 2 \mathrm{H}, \mathrm{CH}_{2}$ ), 3.17 (q, $\left.J=7.3 \mathrm{~Hz}, 18 \mathrm{H}, 9 \times \mathrm{CH}_{2}\right), 3.37-3.44\left(\mathrm{~m}, 2 \mathrm{H}, \mathrm{CH}_{2}\right), 4.69$ (d, $\left.J_{\mathrm{HP}}=4.8 \mathrm{~Hz}, 2 \mathrm{H}, \mathrm{CH}_{2}\right)$, 5.71 (s, $1 \mathrm{H}$ ), 6.58 (s, $1 \mathrm{H}, \mathrm{Ar}$ ), 7.28 (ddd, $J=7.5,4.9$ and $1.1 \mathrm{~Hz}, 1 \mathrm{H}, \mathrm{Ar}$ ), 7.48 (s, $1 \mathrm{H}$, Ar), 7.81 (ddd, $J=8.0,7.5$ and $1.9 \mathrm{~Hz}, 1 \mathrm{H}, \mathrm{Ar}$ ), 8.15-8.20 (m, $1 \mathrm{H}, \mathrm{Ar}$ ), 8.52 (s, $1 \mathrm{H}$, $\mathrm{Ar})$, 8.53-8.57 (m, $1 \mathrm{H}, \mathrm{Ar}) \mathrm{ppm} .{ }^{13} \mathrm{C}$ NMR (100.7 MHz, $\left.\mathrm{CD}_{3} \mathrm{OD}\right): \delta=22.6(-)$, 27.7(+), 30.4(-), 43.7(-), 58.0(-), 64.4(-, d, $\left.J_{\mathrm{CP}}=5.5 \mathrm{~Hz}\right), 96.2(+), 108.9(-), 110.0(-), 116.7(-)$, 118.1(-), 122.2(+), 123.2(+), 123.5(+), 127.3(-, d, $\left.J_{\mathrm{CP}}=7.7 \mathrm{~Hz}\right), 131.1(+), 136.8(+)$, 143.8(+), 148.3(+), 148.7(-), 152.5(-), 156.6(-), 161.6(-), 175.4(-) ppm. HRMS (ESI): calc. for $\mathrm{C}_{24} \mathrm{H}_{25} \mathrm{~N}_{2} \mathrm{O}_{8} \mathrm{P}[\mathrm{M}-\mathrm{H}]^{-}$499.1276; found 499.1266. UV-Vis spectral data in PBS 
7.4: $\lambda_{\mathrm{abs}, \max }=432 \mathrm{~nm} ; \varepsilon=20417 \mathrm{M}^{-1} \mathrm{~cm}^{-1}, \lambda_{\mathrm{em}, \max }=512 \mathrm{~nm}, \Phi_{\mathrm{fl}}=0.81$ (standard: Coumarin 522, $\Phi_{\mathrm{fl}}=0.65$ in EtOH).<smiles>CC1(C)C=C(COP(O)O)c2cc3cc(-c4ccccn4)c(=O)oc3cc2N1CCCC(=O)ON1C(=O)CCC1=O</smiles>

2,5-Dioxopyrrolidin-1-yl 4-(8,8-dimethyl-2-oxo-6-(phosphonooxymethyl)-3-(pyridin2-yl)-1,2,8,9-tetrahydropyrano[3,2-g]quinolin-9-yl)butanoate (309)

Solution of $308 \cdot 3 \mathrm{NEt}_{3}$ (10 mg, $0.012 \mathrm{mmol}$ ), N-hydroxysuccinimide (2.8 mg; 0.024 mmol), HATU (11.4 mg, $0.030 \mathrm{mmol})$ and $\mathrm{NEt}_{3}(12 \mathrm{mg}, 0.120 \mathrm{mmol})$ in DMF (1.5 mL) was stirred overnight at r.t. Afterwards, DMF was evaporated at r.t., and the residue was subjected to column chromatography (15 $\mathrm{g}$ of $\mathrm{SiO}_{2}, \mathrm{MeCN} /$ water, 4:1) to give $3 \mathrm{mg}$ of red solid (42\%). HPLC: $\mathrm{B} / \mathrm{A}=20 / 80$ to $50 / 50$ in $25 \mathrm{~min}$, detection at $433 \mathrm{~nm}, t_{\mathrm{R}}=12.6$ min (100\%). HRMS (ESI): calc. for $\mathrm{C}_{28} \mathrm{H}_{28} \mathrm{~N}_{3} \mathrm{O}_{10} \mathrm{P}[\mathrm{M}-\mathrm{H}]^{-}$596.1440; found 596.1428.<smiles>O=C(O)Cc1ccnc(Br)c1</smiles>

\section{2-(2-bromopyridin-4-yl)acetic acid (316)}

A dried Schlenk flask was charged with a solution of $i \mathrm{Pr}_{2} \mathrm{NH}$ (704 mg, $7.0 \mathrm{mmol}$ ) in THF $(10 \mathrm{~mL})$, cooled to $-78{ }^{\circ} \mathrm{C}$, and $1.6 \mathrm{M}$ solution of BuLi in hexanes $(4.4 \mathrm{~mL}, 7.0 \mathrm{mmol})$ was injected. After $15 \mathrm{~min}$ stirring at this temperature, 2-bromo-4-picoline (1 g, $5.8 \mathrm{mmol}$ ) was added. The reaction mixture was allowed to stir for $1.5 \mathrm{~h}$ and then was quenched with an excess of solid $\mathrm{CO}_{2}$. After the reaction mixture had warmed to r.t., water was added until clear phases formed. $\mathrm{pH}$ was adjusted to 14 with $\mathrm{KOH}$ under icecooling. The resulting mixture was extracted with $\mathrm{CH}_{2} \mathrm{Cl}_{2}(3 \times)$. The aq. layer was separated and acidified to $\mathrm{pH} 3$ with conc. $\mathrm{HCl}$. Further extraction with $\mathrm{CH}_{2} \mathrm{Cl}_{2}(4 \times)$ gave organic solution, which was dried with $\mathrm{Na}_{2} \mathrm{SO}_{4}$ and evaporated at r.t. to furnish $1170 \mathrm{mg}$ (80\%) of the crude product as a hydrochloride. ${ }^{1} \mathrm{H}$ NMR (400 MHz, $\mathrm{CD}_{3} \mathrm{OD}$ ): $\delta=3.69$ (s, $2 \mathrm{H}, \mathrm{CH}_{2}$ ), 7.34 (d, $\left.J=5.1 \mathrm{~Hz}, 1 \mathrm{H}, \mathrm{Ar}\right), 7.58$ (s, $1 \mathrm{H}, \mathrm{Ar}$ ), 8.25 (d, $J=5.1 \mathrm{~Hz}, 1 \mathrm{H}, \mathrm{Ar}$ ) ppm. MS (ESI): $m / z$ (positive mode, rel. int., \%) = $216(100)[\mathrm{M}+\mathrm{H}]^{+}$. 
<smiles>CCOC(=O)CCCN1c2cc3oc(=O)c(-c4ccnc(Br)c4)cc3cc2C(C)=CC1(C)C</smiles>

Ethyl 4-(3-(2-bromopyridin-4-yl)-6,8,8-trimethyl-2-oxo-1,2,8,9-tetrahydropyrano[3,2-g]quinolin-9-yl)butanoate (317)

To a solution of $289(1280 \mathrm{mg}, 3.9 \mathrm{mmol})$ in $\mathrm{CH}_{2} \mathrm{Cl}_{2}(50 \mathrm{~mL})$ compound $316 \cdot \mathrm{HCl}(1170$ mg, $4.6 \mathrm{mmol}), \mathrm{NEt}_{3}(1580 \mathrm{mg}, 15.6 \mathrm{mmol})$, DCC (1200 mg, $\left.5.8 \mathrm{mmol}\right)$ and DMAP (47 mg, $0.39 \mathrm{mmol}, 10 \mathrm{~mol} \%)$ were added. The resulting mixture was stirred overnight. Afterwards, an additional amount of DCC (1200 mg, $5.8 \mathrm{mmol}$ ) was added, and the reaction mixture was heated under reflux for $6 \mathrm{~h}$. After cooling down, the precipitated urea was filtered, and the filtrate was evaporated under reduced pressure. The residue was subjected to column chromatography (150 g of $\mathrm{SiO}_{2}, n$-hexane/EtOAc, 2:1) to furnish $1320 \mathrm{mg}(66 \%)$ of a title product as a yellow-orange powder. ${ }^{1} \mathrm{H}$ NMR (400 MHz, $\left.\mathrm{CDCl}_{3}\right): \delta=1.31$ (t, $\left.J=7.1 \mathrm{~Hz}, 3 \mathrm{H}, \mathrm{Et}\right), 1.41$ (s, $\left.3 \mathrm{H}, 2 \times \mathrm{Me}\right), 1.89-1.98\left(\mathrm{~m}, 2 \mathrm{H}, \mathrm{CH}_{2}\right)$, 2.00 (s, $3 \mathrm{H}, \mathrm{Me}$ ), 2.43 (t, $\left.J=6.8 \mathrm{~Hz}, 2 \mathrm{H}, \mathrm{CH}_{2}\right), 3.31-3.40\left(\mathrm{~m}, 2 \mathrm{H}, \mathrm{NCH}_{2}\right), 4.20$ (q, $J=$ $7.1 \mathrm{~Hz}, 2 \mathrm{H}, \mathrm{Et}$ ), 5.33 (s, $1 \mathrm{H}, \mathrm{Ar}$ ), 6.38 (s, $1 \mathrm{H}, \mathrm{Ar}$ ), 7.10 (s, $1 \mathrm{H}, \mathrm{Ar}$ ), 7.68 (dd, $J=5.3$ and $1.5 \mathrm{~Hz}, 1 \mathrm{H}, \mathrm{Ar}$ ), 7.85 (s, $1 \mathrm{H}, \mathrm{Ar}$ ), 7.88 (d, $J=1.5 \mathrm{~Hz}, 1 \mathrm{H}, \mathrm{Ar}$ ), 8.34 (d, $J=5.3 \mathrm{~Hz}$, $1 \mathrm{H}$, Ar) ppm. HRMS (ESI): calc. for $\mathrm{C}_{26} \mathrm{H}_{25} \mathrm{~N}_{2} \mathrm{O}_{5} \mathrm{Br}[\mathrm{M}+\mathrm{H}]^{+}$511.1227; found 511.1202.<smiles>CCOC(=O)CCCN1c2cc3oc(=O)c(-c4ccnc(-c5ccccc5O)c4)cc3cc2C(C)=CC1(C)C</smiles>

Ethyl 4-(3-(2-(2-hydroxyphenyl)pyridin-4-yl)-6,8,8-trimethyl-2-oxo-1,2,8,9tetrahydropyrano[3,2-g]quinolin-9-yl)butanoate (318)

In a screw-cap tube compound 317 (20 mg, $0.04 \mathrm{mmol}$ ), 2-hydroxyphenylboronic acid (5.5 mg, $0.04 \mathrm{mmol}), \mathrm{Pd}\left(\mathrm{PPh}_{3}\right)_{4}(2.3 \mathrm{mg}, 0.002 \mathrm{mmol}, 5 \mathrm{~mol} \%)$, sat. aq. solution of $\mathrm{Na}_{2} \mathrm{CO}_{3}(80 \mu \mathrm{L}, 2 \mathrm{M}, 0.16 \mathrm{mmol})$, EtOH $(80 \mu \mathrm{L})$ and toluene $(1 \mathrm{~mL})$ were placed under argon. The mixture was heated to $110{ }^{\circ} \mathrm{C}$ and left stirred overnight at this temperature. Afterwards, the reaction mixture was allowed to cool to r.t., diluted with $\mathrm{CH}_{2} \mathrm{Cl}_{2}(10 \mathrm{~mL})$, passed through a plug of Celite (eluting with $\mathrm{CH}_{2} \mathrm{Cl}_{2}$ ), and the filtrate evaporated in vacuo. Column chromatography (25 g of $\mathrm{SiO}_{2}, n$-hexane/EtOAc, 3:1) furnished the title 
product as a yellow solid (17 mg; 81\%). ${ }^{1} \mathrm{H}$ NMR (400 $\mathrm{MHz}, \mathrm{CDCl}_{3}$ ): $\delta=1.33$ (t, $J=$ $7.1 \mathrm{~Hz}, 3 \mathrm{H}, \mathrm{Et}), 1.43$ (s, $6 \mathrm{H}, 2 \times \mathrm{Me}), 1.92-2.01$ (m, 2 H, $\mathrm{CH}_{2}$ ), 2.03 (s, $3 \mathrm{H}, \mathrm{Me}$ ), 2.45 (t, $J=6.9 \mathrm{~Hz}, 2 \mathrm{H}, \mathrm{CH}_{2}$ ), 3.34-3.42 (m, $2 \mathrm{H}, \mathrm{CH}_{2}$ ), 4.22 (q, $J=7.1 \mathrm{~Hz}, 2 \mathrm{H}, \mathrm{Et}$ ), 5.34 (s, $1 \mathrm{H}), 6.43$ (s, $1 \mathrm{H}, \mathrm{Ar}), 6.89-6.96$ (m, $1 \mathrm{H}, \mathrm{Ar}), 7.02-7.07$ (m, $1 \mathrm{H}, \mathrm{Ar}), 7.14$ (s, $1 \mathrm{H}, \mathrm{Ar}$ ), 7.28-7.35 (m, 1 H, Ar), 7.63-7.67 (m, 1 H, Ar), 7.88-7.94 (m, 2 H, Ar), 8.34 (s, 1 H, Ar), 8.49-8.53 (m, 1 H, Ar) ppm. MS (ESI): m/z (positive mode, rel. int., \%) = 525 (100) $[\mathrm{M}+\mathrm{H}]^{+}$.<smiles>CCOC(=O)CCCN1c2cc3oc(=O)c(-c4ccnc(Br)c4)cc3cc2C(C=O)=CC1(C)C</smiles>

Ethyl 4-(3-(2-bromopyridin-4-yl)-6-formyl-8,8-dimethyl-2-oxo-1,2,8,9-tetrahydropyrano[3,2-g]quinolin-9-yl)butanoate (320)

A round-bottomed flask was charged with solution of compound 317 (600 mg, 1.17 $\mathrm{mmol})$ in dioxane $(10 \mathrm{~mL})$ and finely powdered $\mathrm{SeO}_{2}(162 \mathrm{mg}, 1.46 \mathrm{mmol})$. The resulted suspension was heated under reflux for $3.5 \mathrm{~h}$, then water $(10 \mathrm{~mL})$ was added, and the reaction mixture was allowed to cool to r.t. All volatile materials were evaporated; the residue was dissolved in $\mathrm{CH}_{2} \mathrm{Cl}_{2}$, washed with saturated aq. $\mathrm{NaHCO}_{3}$, dried, and evaporated in vacuo. The title compound was isolated as a yellow solid (320 mg, 52\%) by means of column chromatography (100 g of $\left.\mathrm{SiO}_{2}, \mathrm{CH}_{2} \mathrm{Cl}_{2} / \mathrm{Et}_{2} \mathrm{O}, 10: 1\right) .{ }^{1} \mathrm{H}$ NMR (400 $\mathrm{MHz}, \mathrm{CDCl}_{3}$ ): $\delta=1.31$ (t, $J=7.1 \mathrm{~Hz}, 3 \mathrm{H}, \mathrm{Et}$ ), 1.56 (s, $6 \mathrm{H}, 2 \times \mathrm{Me}$ ), 1.90-2.00 (m, $2 \mathrm{H}$, $\mathrm{CH}_{2}$ ), 2.44 (t, $J=6.7 \mathrm{~Hz}, 2 \mathrm{H}, \mathrm{CH}_{2}$ ), 3.40 (m, $2 \mathrm{H}, \mathrm{CH}_{2}$ ), 4.20 (q, $J=7.1 \mathrm{~Hz}, 2 \mathrm{H}, \mathrm{Et}$ ), 6.27 (s, $1 \mathrm{H}$ ), 6.50 (s, $1 \mathrm{H}, \mathrm{Ar}$ ), 7.66 (dd, $J=5.3$ and $1.6 \mathrm{~Hz}, 1 \mathrm{H}, \mathrm{Ar}$ ), 7.90 (dd, $J=1.6$ and $0.6 \mathrm{~Hz}, 1 \mathrm{H}, \mathrm{Ar}$ ), 7.92 (s, $1 \mathrm{H}, \mathrm{Ar}$ ), 8.38 (dd, $J=5.3$ and $0.6 \mathrm{~Hz}, 1 \mathrm{H}, \mathrm{Ar}$ ), 8.55 (s, 1 $\mathrm{H}, \mathrm{Ar}$ ), 9.60 (s, $1 \mathrm{H}, \mathrm{CHO}$ ) ppm. ${ }^{13} \mathrm{C} \mathrm{NMR}\left(100 \mathrm{MHz}, \mathrm{CDCl}_{3}\right): \delta=14.3(+), 22.7(-)$, 28.2(+), 31.1(-), 44.2(-), 58.6(-), 60.8(-), 97.7(+), 109.1(-), 114.1(-), 116.9(-), 121.3(+), 126.3(+), 126.3(+), 130.2(-), 142.6(-), 142.7(+), 145.7(-), 148.2(-), 150.0(+), 152.0(+), 156.9(-), 160.0(-), 172.6(-), 191.6(+) ppm. HRMS (ESI): calc. for $\mathrm{C}_{26} \mathrm{H}_{25} \mathrm{~N}_{2} \mathrm{O}_{5} \mathrm{Br}$ $[\mathrm{M}+\mathrm{H}]^{+}$525.1020; found 525.1006. 
<smiles>CCOC(=O)CCCN1c2cc3cc(-c4ccnc(Br)c4)c(=O)oc3cc2C(CO)=CC1(C)C</smiles>

Ethyl 4-(3-(2-bromopyridin-4-yl)-6-(hydroxymethyl)-8,8-dimethyl-2-oxo-1,2,8,9tetrahydropyrano[3,2-g]quinolin-9-yl)butanoate (321)

To an ice cooled solution of compound 320 (200 mg, $0.38 \mathrm{mmol})$ in a solvent mixture (10 mL, THF/MeOH, 1:1) powder of $\mathrm{CeCl}_{3}(93 \mathrm{mg}, 0.38 \mathrm{mmol}$ ) was added under stirring. After its dissolution, $\mathrm{NaBH}_{4}(14.5 \mathrm{mg}, 0.38 \mathrm{mmol})$ was added in one portion. The reaction mixture was stirred for $5 \mathrm{~min}$, acetone $(5 \mathrm{~mL})$ was added, and the reaction mixture was allowed to warm up to r.t. All volatile materials were evaporated in vacuo, the residue was taken up in water $(10 \mathrm{~mL})$ and extracted with $\mathrm{CHCl}_{3}(3 \times 10 \mathrm{~mL})$. The combined organic liquids were dried and concentrated to give a crude product. The title compound was purified by means of column chromatography (25 g of $\mathrm{SiO}_{2}, \mathrm{CH}_{2} \mathrm{Cl}_{2} / \mathrm{MeOH}$, 25:1) as an orange solid (195 mg, 97\%). ${ }^{1} \mathrm{H}$ NMR (400 MHz, $\left.\mathrm{CDCl}_{3}\right): \delta=1.31$ (t, $J=7.1$ $\mathrm{Hz}, 3 \mathrm{H}, \mathrm{Et}), 1.44$ (s, $6 \mathrm{H}, 2 \times \mathrm{Me}$ ), 1.88-1.98 (m, $2 \mathrm{H}, \mathrm{CH}_{2}$ ), 2.42 (t, $J=6.8 \mathrm{~Hz}, 2 \mathrm{H}$, $\mathrm{CH}_{2}$ ), 3.32-3.39 (m, $2 \mathrm{H}, \mathrm{NCH}_{2}$ ), 4.20 (q, $\left.J=7.1 \mathrm{~Hz}, 2 \mathrm{H}, \mathrm{Et}\right), 4.48$ (s, $2 \mathrm{H}, \mathrm{CH}_{2} \mathrm{OH}$ ), 5.58 (s, $1 \mathrm{H}$ ), 6.42 (s, $1 \mathrm{H}, \mathrm{Ar}), 7.28$ (s, $1 \mathrm{H}, \mathrm{Ar}$ ), 7.66 (dd, $J=5.3$ and $1.6 \mathrm{~Hz}, 1 \mathrm{H}, \mathrm{Ar}$ ), 7.85 (s, $1 \mathrm{H}, \mathrm{Ar}$ ), 7.88 (dd, $J=1.6$ and $0.6 \mathrm{~Hz}, 1 \mathrm{H}, \mathrm{Ar}$ ), 8.34 (dd, $\mathrm{J}=5.3$ and $0.6 \mathrm{~Hz}, 1 \mathrm{H}$, Ar) ppm. ${ }^{13} \mathrm{C}$ NMR (100 MHz, $\left.\mathrm{CDCl}_{3}\right): \delta=14.3(+), 22.6(-), 29.2(+), 31.3(-)$, 44.1(-), 58.2(-), 60.8(-), 62.9(-), 96.8(+), 108.6(-), 115.6(-), 117.9(-), 121.5(+), 123.0(+), 126.1(+), 129.4(-), 130.1(+), 142.4(+), 142.5(-), 146.0(-), 148.8(-), 149.9(+), 156.8(-), 160.2(-), 172.7(-) ppm. HRMS (ESI): calc. for $\mathrm{C}_{26} \mathrm{H}_{27} \mathrm{~N}_{2} \mathrm{O}_{5} \mathrm{Br}[\mathrm{M}+\mathrm{H}]^{+}$527.1176; found 527.1181 .<smiles></smiles>

Ethyl 4-(3-(2-(2-(tert-butoxycarbonyloxy)phenyl)pyridin-4-yl)-6-(hydroxymethyl)8,8-dimethyl-2-oxo-1,2,8,9-tetrahydropyrano[3,2-g]quinolin-9-yl)butanoate (322H,Boc,Et) 
In a screw-cap tube, compound 321 (50 mg, $0.095 \mathrm{mmol}$ ), compound 323 (36 mg, 0.113 mmol), $\mathrm{Pd}\left(\mathrm{PPh}_{3}\right)_{4}(5.5 \mathrm{mg}, 0.005 \mathrm{mmol}, 5 \mathrm{~mol} \%), 2 \mathrm{M} \mathrm{Na}_{2} \mathrm{CO}_{3}(190 \mu \mathrm{L}, 0.38 \mathrm{mmol})$, EtOH $(190 \mu \mathrm{L})$ and toluene $(2 \mathrm{~mL})$ were placed under argon. The mixture was heated to $110^{\circ} \mathrm{C}$ and left stirred overnight at this temperature. Afterwards, the reaction mixture was allowed to cool to r.t., diluted with $\mathrm{CH}_{2} \mathrm{Cl}_{2}(10 \mathrm{~mL})$, passed through a plug of Celite (eluting with $\mathrm{CH}_{2} \mathrm{Cl}_{2}$ ), and the filtrate evaporated in vacuo. Column chromatography (60 g of $\mathrm{SiO}_{2}, \mathrm{CH}_{2} \mathrm{Cl}_{2} / \mathrm{MeOH}, 25: 1$ ) furnished the title product as a yellow solid (26 mg, 43\%). ${ }^{1} \mathrm{H}$ NMR (400 MHz, $\mathrm{CDCl}_{3}$ ): $\delta=1.32$ (t, $\left.J=7.1 \mathrm{~Hz}, 3 \mathrm{H}, \mathrm{Et}\right), 1.38$ (s, $9 \mathrm{H}, t \mathrm{Bu}$ ), 1.44 (s, $6 \mathrm{H}, 2 \times \mathrm{Me}$ ), 1.91-2.00 (m, $2 \mathrm{H}, \mathrm{CH}_{2}$ ), 2.43 (t, $J=6.9 \mathrm{~Hz}, 2 \mathrm{H}, \mathrm{CH}_{2}$ ), 3.33-3.40 (m, $2 \mathrm{H}, \mathrm{CH}_{2}$ ), 4.21 (q, J = 7.1 Hz, $2 \mathrm{H}, \mathrm{Et}$ ), 4.47 (br s., $2 \mathrm{H}, \mathrm{CH}_{2} \mathrm{OH}$ ), 1.21 (s, $1 \mathrm{H}$ ), 6.44 (s, 1 H, Ar), 7.23-7.26 (m, 1 H, Ar), 7.27 (s, 1 H, Ar), 7.34-7.39 (m, 1 H, Ar), 7.41-7.45 (m, $1 \mathrm{H}, \mathrm{Ar}$ ), 7.76 (dd, $J=5.3$ and $1.8 \mathrm{~Hz}, 1 \mathrm{H}, \mathrm{Ar}$ ), 7.78-7.81 (m, $1 \mathrm{H}, \mathrm{Ar}$ ), 7.89 (dd, $J=$ 1.8 and $0.8 \mathrm{~Hz}, 1 \mathrm{H}, \mathrm{Ar}$ ), 7.92 (s, $1 \mathrm{H}, \mathrm{Ar}$ ), 8.71 (dd, $J=5.3$ and $0.8 \mathrm{~Hz}, 1 \mathrm{H}, \mathrm{Ar}$ ) ppm. ${ }^{13} \mathrm{C}$ NMR (100 MHz, $\left.\mathrm{CDCl}_{3}\right): \delta=14.3(+), 22.7(-), 27.5(+), 29.1(+), 31.3(-), 44.1(-)$, 58.1(-), 60.8(-), 62.9(-), 83.3(-), 97.0(+), 108.9(-), 117.7(-), 121.1(+), 121.9(+), 122.7(+), 122.8(+), 126.4(+), 129.5(-), 129.7(+), 130.0(+), 130.9(+), 133.2(-), 141.9(+), 143.6(-), 148.3(-), 148.5(-), 149.7(+), 151.4(-), 156.6(-), 160.5(-), 172.7(-) ppm. HRMS (ESI): calc. for $\mathrm{C}_{37} \mathrm{H}_{40} \mathrm{~N}_{2} \mathrm{O}_{8}[\mathrm{M}+\mathrm{H}]^{+}$641.2857; found 641.2855.<smiles>CCCOCC1=CC(C)(C)N(CCCOCC)c2cc3cc(-c4ccnc(-c5ccccc5OCCC)c4)c(=O)oc3cc21</smiles>

Ethyl 4-(3-(2-(2-(tert-butoxycarbonyloxy)phenyl)pyridin-4-yl)-6-((di-tert-butoxyphosphoryloxy)methyl)-8,8-dimethyl-2-oxo-1,2,8,9-tetrahydropyrano[3,2-g]quinolin9-yl)butanoate (322-OPO(tBu) 2 ,Boc,Et)

To a stirred and preheated $\left(40{ }^{\circ} \mathrm{C}\right)$ solution of compound 322-H,Boc,Et (21 mg, $0.033 \mathrm{mmol}$ ) in $\mathrm{CH}_{2} \mathrm{Cl}_{2}(5 \mathrm{~mL})$ di-t-butyl $N, N$-diisopropylphosphoramidite (27 $\mathrm{mg}$, $0.100 \mathrm{mmol}$ ) and $1 \mathrm{H}$-tetrazole (7.4 mg, $0.107 \mathrm{mmol}$ ) were added in two equal portions at interval of 20 min under argon. After further 20 min the reaction mixture was cooled with ice bath $\left(0^{\circ} \mathrm{C}\right.$ ), and solution of $m$ CPBA (24 mg, 70\% purity, $0.100 \mathrm{mmol}$ ) in $\mathrm{CH}_{2} \mathrm{Cl}_{2}$ was added. After stirring for additional 30 min aqueous solutions of $\mathrm{Na}_{2} \mathrm{SO}_{3}(2 \mathrm{~mL}, 10 \%)$ and $\mathrm{NaHCO}_{3}$ (1 mL, saturated) were added, and the reaction mixture was allowed to warm up to r.t. The organic layer was separated and the aqueous phase was extracted with $\mathrm{CH}_{2} \mathrm{Cl}_{2}$ 
$(3 \times 10 \mathrm{~mL})$. The combined organic extracts were dried, the solvents were evaporated, and the titled compound was isolated by column chromatography (30 g of $\mathrm{SiO}_{2}$, $\mathrm{CH}_{2} \mathrm{Cl}_{2} / \mathrm{MeOH}, 25: 1$ ) as a yellow amorphous solid (12 mg; 44\%). ${ }^{1} \mathrm{H}$ NMR (400 MHz, $\mathrm{CDCl}_{3}$ ): $\delta=1.31$ (t, $\left.J=7.1 \mathrm{~Hz}, 3 \mathrm{H}, \mathrm{Et}\right), 1.39$ (s, $\left.9 \mathrm{H}, t \mathrm{Bu}\right), 1.44$ (s, $6 \mathrm{H}, 2 \times \mathrm{Me}$ ), 1.50 (d, $\left.J_{\mathrm{CP}}=0.5 \mathrm{~Hz}, 18 \mathrm{H}, 2 \times t \mathrm{Bu}\right), 1.90-1.99\left(\mathrm{~m}, 2 \mathrm{H}, \mathrm{CH}_{2}\right), 2.43\left(\mathrm{t}, J=6.9 \mathrm{~Hz}, 2 \mathrm{H}, \mathrm{CH}_{2}\right)$, 3.33-3.39 (m, 2 H, NCH 2$), 4.20$ (q, $J=7.1 \mathrm{~Hz}, 2 \mathrm{H}, \mathrm{Et}$ ), 4.75 (d, $J_{\mathrm{CP}}=6.6 \mathrm{~Hz}, 2 \mathrm{H}, \mathrm{CH}_{2}$ ), 5.65 (s, 1 H), 6.44 (s, 1 H), 7.23-7.26 (m, 2 H, Ar), 7.34-7.39 (m, 1 H, Ar), 7.40-7.45 (m, $1 \mathrm{H}, \mathrm{Ar}$ ), 7.72 (dd, $J=5.2$ and $1.7 \mathrm{~Hz}, 1 \mathrm{H}, \mathrm{Ar}$ ), 7.77-7.82 (m, $1 \mathrm{H}, \mathrm{Ar}$ ), 7.89-7.91 (m, 2 $\mathrm{H}, \mathrm{Ar}$ ), 8.71 (dd, $J=5.3$ and $0.8 \mathrm{~Hz}, 1 \mathrm{H}, \mathrm{Ar}$ ) ppm. MS (ESI): $\mathrm{m} / \mathrm{z}$ (positive mode, rel. int., \%) $=833(100)[\mathrm{M}+\mathrm{H}]^{+}$.<smiles></smiles>

Ethyl 4-(3-(2-(1H-pyrrol-2-yl)pyridin-4-yl)-6-(hydroxymethyl)-8,8-dimethyl-2-oxo1,2,8,9-tetrahydropyrano[3,2-g]quinolin-9-yl)butanoate (325)

In a screw-cap tube, compound 321 (20 mg, $0.038 \mathrm{mmol}$ ), compound 326 (10 mg, $0.046 \mathrm{mmol}$ ), $\mathrm{Pd}\left(\mathrm{PPh}_{3}\right)_{4}$ (2.2 mg, $\left.0.002 \mathrm{mmol}, 5 \mathrm{~mol} \%\right)$, sat. aq. solution of $\mathrm{Na}_{2} \mathrm{CO}_{3}$ (76 $\mu \mathrm{L}, 2 \mathrm{M}, 0.152 \mathrm{mmol})$, EtOH $(76 \mu \mathrm{L})$ and toluene $(1.5 \mathrm{~mL})$ were placed under argon. The mixture was stirred for $15 \mathrm{~h}$ at $110{ }^{\circ} \mathrm{C}$ and for $10 \mathrm{~min}$ at $150{ }^{\circ} \mathrm{C}$. Afterwards, the reaction mixture was allowed to cool to r.t., diluted with $\mathrm{CH}_{2} \mathrm{Cl}_{2}(10 \mathrm{~mL})$, passed through a plug of Celite (eluting with $\mathrm{CH}_{2} \mathrm{Cl}_{2}$ ), and the filtrate evaporated in vacuo. Column chromatography (60 g of $\mathrm{SiO}_{2} ; \mathrm{CH}_{2} \mathrm{Cl}_{2} / \mathrm{MeOH}, 25: 1$ ) furnished the title product as an orange solid (12 mg, 61\%). ${ }^{1} \mathrm{H}$ NMR (400 MHz, $\mathrm{CDCl}_{3}$ ): $\delta=1.32$ (t, $J=7.1 \mathrm{~Hz}, 3 \mathrm{H}, \mathrm{Et}$ ), 1.43 (s, $6 \mathrm{H}, 2 \times \mathrm{Me}$ ), 1.90-1.99 (m, $2 \mathrm{H}, \mathrm{CH}_{2}$ ), 2.43 (t, $J=6.9 \mathrm{~Hz}, 2 \mathrm{H}, \mathrm{CH}_{2}$ ), 3.31-3.38 (m, 2 H, $\mathrm{NCH}_{2}$ ), 4.20 (q, $J=7.1 \mathrm{~Hz}, 2 \mathrm{H}, \mathrm{Et}$ ), 4.49 (d, $J=0.9 \mathrm{~Hz}, 2 \mathrm{H}, \mathrm{CH}_{2}$ ), 5.56 (s, $\left.1 \mathrm{H}\right), 6.30$ (m, 1 H, Ar), 6.42 (s, 1 H, Ar), 6.79 (m, 1 H, Ar), 6.90 (m, 1 H, Ar), 7.28 (s, 1 H, Ar), 7.43 (dd, $J=5.3$ and $1.7 \mathrm{~Hz}, 1 \mathrm{H}, \mathrm{Ar}$ ), 7.82 (s, $1 \mathrm{H}, \mathrm{Ar}$ ), 7.88 (dd, $J=1.7$ and $0.8 \mathrm{~Hz}, 1 \mathrm{H}$, Ar), 8.44 (dd, $J=5.3$ and $0.8 \mathrm{~Hz}, 1 \mathrm{H}, \mathrm{Ar}$ ), 9.77 (br. s, $1 \mathrm{H}, \mathrm{Ar}$ ) ppm. ${ }^{13} \mathrm{C} \mathrm{NMR}$ $\left(100 \mathrm{MHz}, \mathrm{CDCl}_{3}\right): \delta=14.3(+), 22.7(-), 29.1(+), 31.3(-), 44.1(-), 58.1(-), 60.8(-)$, 63.0(-), 96.9(+), 107.5(+), 108.8(-), 110.2(+), 116.6(+), 117.7(-), 119.3(+), 119.9(+), 122.8(+), 129.6(-), 130.1(+), 131.5(-), 141.7(+), 143.8(-), 148.3(-), 148.7(+), 150.7(-), 156.6(-), 160.6(-), 172.7(-) ppm. MS (ESI): m/z (positive mode, rel. int., \%) = 514 (100) 
$[\mathrm{M}+\mathrm{H}]^{+}$. UV-Vis spectral data in MeOH: $\lambda_{\text {abs,max }}=432 \mathrm{~nm} ; \varepsilon=33650 \mathrm{M}^{-1} \mathrm{~cm}^{-1}, \lambda_{\text {em,max }}=$ $432 \mathrm{~nm}, \Phi_{\mathrm{fl}}=0.50$ (standard: Coumarin 510, $\Phi_{\mathrm{fl}}=0.85$ in EtOH).<smiles></smiles>

10-(9-(4-Ethoxy-4-oxobutyl)-6-(hydroxymethyl)-8,8-dimethyl-2-oxo-1,2,8,9tetrahydropyrano[3,2-g]quinolin-3-yl)pyrido[1,2-a]pyrrolo[2,1-c]pyrazin-7-ium bromide (328)

In a screw-cap tube a solution of compound 325 (33 $\mathrm{mg}, 0.064 \mathrm{mmol}$ ) in dibromoethane $(1 \mathrm{~mL})$ was stirred at $130{ }^{\circ} \mathrm{C}$ for 3 days. Afterwards, all volatiles were evaporated in vacuo, and the residue was subjected to column chromatography (35 g of $\mathrm{SiO}_{2}$, $\left.\mathrm{CH}_{2} \mathrm{Cl}_{2} / \mathrm{MeOH}, 12: 1 \rightarrow 5: 1\right)$ to furnish $1 \mathrm{mg}(2.5 \%)$ of the title compound. ${ }^{1} \mathrm{H}$ NMR (400 MHz, DMSO-d6): $\delta=1.22$ (t, $J=7.1 \mathrm{~Hz}, 3 \mathrm{H}, \mathrm{Et}$ ), 1.41 (s, $6 \mathrm{H}, 2 \times \mathrm{Me}$ ), 1.74-1.84 (m, 2 $\mathrm{H}, \mathrm{CH}_{2}$ ), 2.51 (t, $J=6.8 \mathrm{~Hz}, 2 \mathrm{H}, \mathrm{CH}_{2}$ ), 3.40-3.48 (m, $2 \mathrm{H}, \mathrm{NCH}_{2}$ ), 4.11 (q, $J=7.1 \mathrm{~Hz}, 2$ H, Et), 4.28 (s, 2 H, $\mathrm{CH}_{2} \mathrm{OH}$ ), 5.68 (s, $1 \mathrm{H}$ ), 6.68 (s, $1 \mathrm{H}, \mathrm{Ar}$ ), 7.12 (dd, $J=4.1$ and 2.6 Hz, 1 H, Ar), 7.43 (s, 1 H, Ar), 7.88 (d, $J=4.1$ Hz, 1 H, Ar), 8.03-8.06 (m, 2 H, Ar), 8.36 (dd, $J=7.2$ and $2.1 \mathrm{~Hz}, 1 \mathrm{H}, \mathrm{Ar}$ ), 8.56 (d, $J=5.9 \mathrm{~Hz}, 1 \mathrm{H}, \mathrm{Ar}$ ), 8.83 (s, $1 \mathrm{H}, \mathrm{Ar}$ ), 8.93 (d, $J=7.3 \mathrm{~Hz}, 1 \mathrm{H}, \mathrm{Ar}), 9.04$ (d, $J=2.0 \mathrm{~Hz}, 1 \mathrm{H}, \mathrm{Ar}) \mathrm{ppm} .{ }^{13} \mathrm{C}$ NMR (100.5 MHz, DMSO$d 6): \delta=14.6(+), 22.7(-), 29.3(+), 30.8(-), 44.0(-), 59.2(-), 60.5(-), 61.0(-), 96.4(+)$, 109.0(-), 110.0(+), 111.4(-), 116.5(+), 116.7(+), 116.8(+), 118.6(-), 119.7(+), 121.4(+), 122.2(-), 122.6(+), 124.1(+), 129.4(-), 129.7(+), 137.4(+), 137.8(-), 146.2(+), 147.7(-), 150.4(-), 157.7(-), 159.8(-), 173.2(-) ppm. HRMS (ESI): calc. for $\mathrm{C}_{32} \mathrm{H}_{32} \mathrm{~N}_{3} \mathrm{O}_{5} \mathrm{M}^{+}$ 538.2336; found 538.2326. UV-Vis spectral data in $\mathrm{MeOH}: \lambda_{\mathrm{abs}, \max }=489 \mathrm{~nm}, \lambda_{\mathrm{em}, \max }=$ $587 \mathrm{~nm}, \Phi_{\mathrm{fl}}=0.56$ (standard: Rhodamine B, $\Phi_{\mathrm{fl}}=0.69$ in EtOH).<smiles>CCOC(=O)CCCN1CN2c3cc4cc1c(-c1cc[n+]5c(c1)-c1cccn1C(O)C5)cc4cc3C(CO)=CC2(C)C</smiles> 


\section{0-(9-(4-Ethoxy-4-oxobutyl)-6-(hydroxymethyl)-8,8-dimethyl-2-oxo-1,2,8,9-}

tetrahydropyrano[3,2-g]quinolin-3-yl)-5-hydroxy-5,6-dihydropyrido[1,2-

a]pyrrolo[2,1-c]pyrazin-7-ium chloride (329)

In a screw-cap tube, a mixture of compound 325 (17 mg, $0.033 \mathrm{mmol})$, chloroacetaldehyde diethyl acetal $(1 \mathrm{~mL})$ and $\mathrm{NaI}(100 \mathrm{mg}, 0.66 \mathrm{mmol})$ in DMF $(1 \mathrm{~mL})$ was stirred for $5 \mathrm{~h}$ at $100{ }^{\circ} \mathrm{C}$. Afterwards, the reaction mixture was diluted with water $(\sim 20$ $\mathrm{mL}$ ), and sat. aq. $\mathrm{Na}_{2} \mathrm{~S}_{2} \mathrm{O}_{3}$ was added $(\sim 5 \mathrm{~mL})$. The resulting slurry was extracted with $\mathrm{CHCl}_{3}(3 \times 25 \mathrm{~mL})$, and combined organic extracts were washed with sat. aq. $\mathrm{NaCl}$, dried with $\mathrm{Na}_{2} \mathrm{SO}_{4}$ and evaporated. The residue was subjected to column chromatography (30 $\mathrm{g}$ of $\left.\mathrm{SiO}_{2}, \mathrm{CH}_{2} \mathrm{Cl}_{2} / \mathrm{MeOH}, 5: 1 \rightarrow 2.5: 1\right)$ to give $12 \mathrm{mg}(67 \%)$ of a purple solid. ${ }^{1} \mathrm{H}$ NMR (400 MHz, DMSO-d6): $\delta=1.21$ (t, J = 7.1 Hz, $3 \mathrm{H}, \mathrm{Et}$ ), 1.40 (s, $6 \mathrm{H}, 2 \times \mathrm{Me}$ ), 1.73-1.83 (m, $2 \mathrm{H}, \mathrm{CH}_{2}$ ), 2.50 (t, $J=6.7 \mathrm{~Hz}, 2 \mathrm{H}, \mathrm{CH}_{2}$ ), 3.39-3.45 (m, $2 \mathrm{H}, \mathrm{NCH}_{2}$ ), 4.11 (q, $J=7.1$ Hz, 2 H, Et), 4.27 (br. s, 2 H, CH${ }_{2} \mathrm{OH}$ ), 4.79-4.84 (m, 2 H, $\mathrm{CH}_{2}$ ), 5.67 (s, $1 \mathrm{H}$ ), 6.08-6.12 (m, $1 \mathrm{H}$ ), 6.47 (dd, $J=3.4$ and $2.6 \mathrm{~Hz}, 1 \mathrm{H}, \mathrm{Ar}$ ), 6.66 (s, $1 \mathrm{H}, \mathrm{Ar}$ ), 7.40-7.45 (m, $3 \mathrm{H}, \mathrm{Ar}$ ), 8.17 (dd, $J=7.0$ and $2.1 \mathrm{~Hz}, 1 \mathrm{H}, \mathrm{Ar}$ ), 8.65 (d, $J=2.1 \mathrm{~Hz}, 1 \mathrm{H}, \mathrm{Ar}$ ), 8.70 (d, J = 7.0 Hz, 1 $\mathrm{H}, \mathrm{Ar}$ ), 8.81 (s, $1 \mathrm{H}, \mathrm{Ar}$ ) ppm. ${ }^{13} \mathrm{C}$ NMR (100.5 MHz, DMSO-d6): $\delta=14.6(+), 22.7(-)$, 29.3(+), 30.8(-), 44.0(-), 58.8(-), 59.1(-), 60.5(-), 60.9(-), 73.8(+), 96.4(+), 108.9(-), 111.2(-), 112.6(+), 115.6(+), 117.8(+), 118.6(-), 119.7(+), 120.6(-), 124.1(+), 127.2(+), 129.4(-), 129.6(+), 142.2(-), 143.3(+), 146.4(+), 150.4(-), 150.8(-), 157.8(-), 159.8(-), 173.2(-) ppm. HRMS (ESI): calc. for $\mathrm{C}_{32} \mathrm{H}_{34} \mathrm{~N}_{3} \mathrm{O}_{6}[\mathrm{M}]^{+}$556.2442; found 556.2439.<smiles>COc1cc2c(cc1Br)C(C)=CC(C)(C)N2</smiles>

\section{6-Bromo-7-methoxy-2,2,4-trimethyl-1,2-dihydroquinoline (335)}

Anhydrous ytterbium(III) triflate $(1.0 \mathrm{~g}, 1.63 \mathrm{mmol}, 6.5 \mathrm{~mol} \%$, freshly dried in vacuo at $130{ }^{\circ} \mathrm{C}$ for $4 \mathrm{~h}$ ) was added in one portion to a solution of compound 334 (5.0 g, $25 \mathrm{mmol}$ ) in dry acetone $(75 \mathrm{~mL})$. The reaction mixture was stirred at r.t. for $16 \mathrm{~h}$. Acetone was evaporated in vacuo, the residue was dissolved in EtOAc, washed with sat. aq. $\mathrm{NaHCO}_{3}$ (twice), water, brine and dried over $\mathrm{MgSO}_{4}$. After evaporation of solvents, the oily residue was dried in vacuo (0.5 Torr) to a constant weight. Purification by column chromatography (100 g of $\mathrm{SiO}_{2}, n$-hexane/ $\mathrm{Et}_{2} \mathrm{O}, 4: 1$ ) afforded the title compound a white powder (5.6 g, 80\% yield). ${ }^{1} \mathrm{H}$ NMR (300 MHz, $\mathrm{CDCl}_{3}$ ): $\delta=1.25$ (s, $6 \mathrm{H}, 2 \times \mathrm{Me}$ ), 1.91 (d, $J=$ $1.31 \mathrm{~Hz}, 3 \mathrm{H}, \mathrm{Me}$ ), 3.80 (s, 3 H, OMe), 5.18 (br. s, 1 H), 6.02 (s, 1 H, Ar), 7.13 (s, 1 H, 
Ar) ppm. ${ }^{13} \mathrm{C}$ NMR $\left(75 \mathrm{MHz}, \mathrm{CDCl}_{3}\right): \delta=18.5(+)$, 31.0(+), 52.1(-), 56.1(+), 97.2(+), 97.8(-), 116.2(-), 126.5(+), 127.4(-), 128.0(+), 143.8(-), 155.7(-) ppm. HRMS (ESI): found 304.0304; calc. for $\mathrm{C}_{13} \mathrm{H}_{16} \mathrm{NOBr}[\mathrm{M}+\mathrm{Na}]^{+} 304.0307$.<smiles>CCOC(=O)CCCN1c2cc(OC)c(Br)cc2C(C)=CC1(C)C</smiles>

Ethyl 4-(6-bromo-7-methoxy-2,2,4-trimethylquinolin-1(2H)-yl)butanoate (336)

DIEA (3.4 g, 26.0 mmol) was added to a mixture of compound 335 (5.6 g, $20.0 \mathrm{mmol}$ ) and ethyl 3-iodobutyrate (6.3 g, $26.0 \mathrm{mmol}$ ) in a screw-cup bottle, and the reaction mixture was stirred with heating $\left(110{ }^{\circ} \mathrm{C}\right)$ for $64 \mathrm{~h}$. After cooling down, the reaction mixture was distributed between EtOAc $(70 \mathrm{~mL})$ and water $(70 \mathrm{~mL})$. The organic layer was washed with brine $(70 \mathrm{~mL})$ and water $(70 \mathrm{~mL})$, dried with $\mathrm{Na}_{2} \mathrm{SO}_{4}$ and evaporated. The residue was subjected to column chromatography (150 g of $\mathrm{SiO}_{2}, n$-hexane/ $\mathrm{CH}_{2} \mathrm{Cl}_{2}, 3: 5$ ) to furnish $6.5 \mathrm{~g}(82 \%)$ of the title compound. ${ }^{1} \mathrm{H}$ NMR (300 MHz, $\left.\mathrm{CDCl}_{3}\right): \delta=1.25$ (t, $J=$ $7.1 \mathrm{~Hz}, 3 \mathrm{H}, \mathrm{Et}), 1.27$ (s, $6 \mathrm{H}, 2 \times \mathrm{Me}$ ), 1.84-1.95 (m, $2 \mathrm{H}, \mathrm{CH}_{2}$ ), 1.89 (d, $J=1.4 \mathrm{~Hz}, 3 \mathrm{H}$, Me), 2.37 (t, $J=6.7 \mathrm{~Hz}, 2 \mathrm{H}, \mathrm{CH}_{2}$ ), 3.19-3.26 (m, $2 \mathrm{H}, \mathrm{NCH}_{2}$ ), 3.91 (s, $\left.3 \mathrm{H}, \mathrm{OMe}\right), 4.13$ (q, $J=7.1 \mathrm{~Hz}, 2 \mathrm{H}, \mathrm{Et}$ ), 5.09 (q, $J=1.3 \mathrm{~Hz}, 1 \mathrm{H}$ ), 6.28 (s, $1 \mathrm{H}, \mathrm{Ar}$ ), 7.10 (s, $1 \mathrm{H}, \mathrm{Ar}$ ) ppm. ${ }^{13} \mathrm{C} \mathrm{NMR}\left(75 \mathrm{MHz}, \mathrm{CDCl}_{3}\right): \delta=14.2(+), 18.6(+), 23.2(-), 28.3(+), 31.3(-)$, 43.5(-), 56.4(+), 57.0(-), 60.5(-), 96.3(+), 96.6(-), 117.7(-), 126.8(-), 127.6(+), 127.7(+), 144.5(), 156.1(-), 173.1(-) ppm. HRMS (ESI): found 396.1165; calc. for $\mathrm{C}_{19} \mathrm{H}_{26} \mathrm{NO}_{3} \mathrm{Br}[\mathrm{M}+\mathrm{H}]^{+}$ 396.1169.<smiles>COc1cc2c(cc1B(F)F)C(C)=CC(C)(C)N2CCCC[In]</smiles>

7-Methoxy-2,2,4-trimethyl-1-(4-(tetrahydro-2H-pyran-2-yloxy)butyl)-6-(4,4,5,5tetramethyl-1,3,2-dioxaborolan-2-yl)-1,2-dihydroquinoline (338)

In a dried Schlenk flask a solution of compound 339-THP (500 mg, $1.14 \mathrm{mmol}$ ) in THF (20 mL) was placed under argon. The flask was cooled down to $-78{ }^{\circ} \mathrm{C}$, and a solution of $t \operatorname{BuLi}(740 \mu \mathrm{L}, 1.7 \mathrm{M}, 1.26 \mathrm{mmol})$ was injected with stirring. After $45 \mathrm{~min}$ stirring at -78 ${ }^{\circ} \mathrm{C}, \mathrm{B}(\mathrm{OiPr})_{3}$ (237 mg, $1.26 \mathrm{mmol}$ ) was added. The resulting mixture was stirred over- 
night at r.t., and then AcOH (76 mg, $1.26 \mathrm{mmol})$ and pinacol (297 mg, $2.52 \mathrm{mmol})$ were added. After $1 \mathrm{~h}$ stirring, brine (20 mL) was added thereto, the organic layer was separated, and the aq. layer was extracted with EtOAc $(2 \times 20 \mathrm{~mL})$. Combined organic solutions were dried with $\mathrm{Na}_{2} \mathrm{SO}_{4}$ and evaporated. The residue was purified by column chromatography (50 g of $\mathrm{SiO}_{2}, n$-hexane/EtOAc, 2:1) to yield $490 \mathrm{mg}$ (89\%) of a yellow oil. ${ }^{1} \mathrm{H}$ NMR (300 MHz, $\mathrm{CDCl}_{3}$ ): $\delta=1.28$ (s, $6 \mathrm{H}, 2 \times \mathrm{Me}$ ), 1.29 (s, $12 \mathrm{H}, 4 \times \mathrm{Me}$ ), 1.44 1.58 (m, 4 H, 2× $\mathrm{CH}_{2}$ ), 1.59-1.82 (m, $6 \mathrm{H}, 3 \times \mathrm{CH}_{2}$ ), 1.97 (s, $3 \mathrm{H}, \mathrm{Me}$ ), 3.21-3.29 (m, $2 \mathrm{H}$, $\mathrm{CH}_{2}$ ), 3.35-3.52 (m, $2 \mathrm{H}, \mathrm{OCH}_{2}$ ), 3.72-3.78 (m, 2 H, NCH 2 ), 3.80 (s, $\left.3 \mathrm{H}, \mathrm{OMe}\right), 4.52-$ 4.58 (m, $1 \mathrm{H}$ ), 5.03 (br. s, $1 \mathrm{H}), 5.95$ (s, $1 \mathrm{H}, \mathrm{Ar}), 7.35$ (s, $1 \mathrm{H}, \mathrm{Ar}) \mathrm{ppm} .{ }^{13} \mathrm{C} \mathrm{NMR}$ (75 MHz, $\left.\mathrm{CDCl}_{3}\right): \delta=18.3(+), 19.6(-)$, 24.8(+), 25.4(-), 25.5(-), 27.5(-), 28.9(+), 30.7(), 44.1(-), 56.1(+), 57.1(-), 62.4(-), 67.2(-), 82.6(-), 98.9(+), 115.5(-), 125.9(+), 127.7(), 132.5(+), 148.0(-), 166.2(-) ppm. HRMS (ESI): found 508.3212; calc. for $\mathrm{C}_{28} \mathrm{H}_{44} \mathrm{NO}_{5} \mathrm{~B}$ $[\mathrm{M}+\mathrm{Na}]^{+} 508.3210$.<smiles>COc1cc2c(cc1Br)C(C)=CC(C)(C)N2CCCCO</smiles>

\section{4-(6-Bromo-7-methoxy-2,2,4-trimethylquinolin-1(2H)-yl)butan-1-ol (339-H)}

To a suspension of $\mathrm{LiAlH}_{4}$ (38 mg, $1 \mathrm{mmol}$ ) in THF (5 mL) a solution of compound 336 (500 mg, $1.26 \mathrm{mmol}$ ) in THF (5 mL) was added dropwise at $0{ }^{\circ} \mathrm{C}$. The resulting mixture was stirred for $1.5 \mathrm{~h}$ at $0{ }^{\circ} \mathrm{C}$ and for $1 \mathrm{~h}$ at r.t. Afterwards, the reaction mixture was quenched with $1 \mathrm{M} \mathrm{KHSO}_{4}(5 \mathrm{~mL})$, brine $(5 \mathrm{~mL})$ was added, and the organic layer was separated. The aq. layer was extracted with EtOAc $(3 \times 10 \mathrm{~mL})$, and the combined organic solutions were dried with $\mathrm{Na}_{2} \mathrm{SO}_{4}$ and evaporated. The residue was subjected to column chromatography (50 g of $\mathrm{SiO}_{2}, n$-hexane/EtOAc, 1:1) to afford $440 \mathrm{mg}$ (98\%) of a yellow oil. ${ }^{1} \mathrm{H}$ NMR (300 MHz, $\left.\mathrm{CDCl}_{3}\right): \delta=1.27$ (s, $6 \mathrm{H}, 2 \times \mathrm{Me}$ ), 1.57-1.73 (m, $4 \mathrm{H}, 2 \times \mathrm{CH}_{2}$ ), 1.89 (d, $J=1.2 \mathrm{~Hz}, 3 \mathrm{H}, \mathrm{Me}), 3.18-3.25$ (m, $\left.2 \mathrm{H}, \mathrm{OCH}_{2}\right), 3.65-3.71$ (m, $2 \mathrm{H}, \mathrm{NCH}_{2}$ ), 3.85 (s, 3 H, OMe), 5.10 (br. s, $1 \mathrm{H}$ ), 6.05 (s, $1 \mathrm{H}, \mathrm{Ar}$ ), 7.10 (s, $1 \mathrm{H}, \mathrm{Ar}$ ) ppm. ${ }^{13} \mathrm{C} \mathrm{NMR}$ (75 MHz, $\left.\mathrm{CDCl}_{3}\right): \delta=18.6(+), 24.7(-), 28.4(+), 30.2(-), 44.0(-)$, 56.1(+), 60.4(-), 62.5(-), 96.0(+), 96.4(-), 117.8(-), 126.7(-), 127.7(+), 127.7(+), 144.4(-), 155.9(-) ppm. HRMS (ESI): found 376.0881; calc. for $\mathrm{C}_{17} \mathrm{H}_{24} \mathrm{NO}_{2} \mathrm{Br}[\mathrm{M}+\mathrm{Na}]^{+} 376.0883$. 
<smiles>COc1cc2c(cc1Br)C(C)=CC(C)(C)N2CCCC[OH+]</smiles>

6-Bromo-7-methoxy-2,2,4-trimethyl-1-(4-(tetrahydro-2H-pyran-2-yloxy)butyl)-1,2dihydroquinoline (339-THP)

To a solution of compound 339-H (100 mg, $0.28 \mathrm{mmol})$ in $\mathrm{CH}_{2} \mathrm{Cl}_{2}$ (2 mL) 3,4-dihydro$2 \mathrm{H}$-pyran (105 mg, $1.25 \mathrm{mmol})$ and $\mathrm{TsOH} \cdot \mathrm{H}_{2} \mathrm{O}(0.5 \mathrm{mg}, 0.025 \mathrm{mmol})$ were added at 0 ${ }^{\circ} \mathrm{C}$. The resulting mixture was stirred overnight at r.t. Afterwards, additional amounts of 3,4-dihydro-2H-pyran (105 mg, $1.25 \mathrm{mmol}$ ) and $\mathrm{TsOH} \cdot \mathrm{H}_{2} \mathrm{O}$ (5 mg, $0.25 \mathrm{mmol}$ ) were added, and the reaction mixture was heated to $40{ }^{\circ} \mathrm{C}$ and stirred for $20 \mathrm{~min}$ at this temperature. After cooling down, the reaction mixture was diluted to $5 \mathrm{~mL}$ with $\mathrm{CH}_{2} \mathrm{Cl}_{2}$, and sat. aq. $\mathrm{NaHCO}_{3}(5 \mathrm{~mL})$ was added. The organic layer was separated, and the aq. layer was extracted with $\mathrm{CH}_{2} \mathrm{Cl}_{2}(2 \times 5 \mathrm{~mL})$. Combined organic solutions were dried with $\mathrm{Na}_{2} \mathrm{SO}_{4}$ and evaporated. The residue was subjected to column chromatography (20 g of $\mathrm{SiO}_{2}, n$-hexane/EtOAc, 5:1) to give $102 \mathrm{mg}$ (82\%) of a colorless oil. ${ }^{1} \mathrm{H}$ NMR (300 MHz, $\left.\mathrm{CDCl}_{3}\right): \delta=1.27(\mathrm{~s}, 6 \mathrm{H}, 2 \times \mathrm{Me}), 1.44-1.58\left(\mathrm{~m}, 4 \mathrm{H}, 2 \times \mathrm{CH}_{2}\right), 1.61-1.74(\mathrm{~m}, 6 \mathrm{H}$, $3 \times \mathrm{CH}_{2}$ ), 1.89 (br. s, $3 \mathrm{H}, \mathrm{Me}$ ), 3.16-3.25 (m, $2 \mathrm{H}, \mathrm{CH}_{2}$ ), 3.36-3.52 (m, $2 \mathrm{H}, \mathrm{CH}_{2}$ ), 3.743.82 (m, 2 H, $\mathrm{CH}_{2}$ ), 3.85 (s, $3 \mathrm{H}, \mathrm{OMe}$ ), 4.55 (t, $J=3.4 \mathrm{~Hz}, 1 \mathrm{H}$ ), 5.09 (br. s, $1 \mathrm{H}$ ), 6.02 (s, $1 \mathrm{H}, \mathrm{Ar}), 7.09$ (s, $1 \mathrm{H}, \mathrm{Ar}) \mathrm{ppm} .{ }^{13} \mathrm{C} \mathrm{NMR}\left(125.7 \mathrm{MHz}, \mathrm{CDCl}_{3}\right): \delta=18.5(+), 19.6(-)$, 25.2(-), 25.3(-), 27.4(-), 28.4(+), 30.7(-), 44.2(-), 56.1(+), 57.0(-), 62.5(-), 67.2(-), 96.0(+), 96.3(-), 99.0(+), 117.9(-), 126.8(-), 127.8(+), 144.6(-), 156.0(-) ppm. HRMS (ESI): found 460.1451; calc. for $\mathrm{C}_{22} \mathrm{H}_{32} \mathrm{NO}_{3} \mathrm{Br}[\mathrm{M}+\mathrm{Na}]^{+} 460.1458$.<smiles>COc1cc2c(cc1-c1ccnc(Cl)c1C(=O)O)C(C)=CC(C)(C)N2CCCCO</smiles>

\section{2-Chloro-4-(7-methoxy-2,2,4-trimethyl-1-(4-(tetrahydro-2H-pyran-2-yloxy)butyl)-}

\section{1,2-dihydroquinolin-6-yl)nicotinic acid (342-THP)}

In a screw-cap tube, compound 339-THP (490 mg, $1.01 \mathrm{mmol}$ ), 2-chloro-4-iodonicotinic acid (314 mg, $1.11 \mathrm{mmol}$ ), $\mathrm{Pd}\left(\mathrm{PPh}_{3}\right)_{4}$ (58 mg, $0.05 \mathrm{mmol}, 5 \mathrm{~mol} \%$ ), sat. aq. solution of $\mathrm{Na}_{2} \mathrm{CO}_{3}(2 \mathrm{~mL}, 2 \mathrm{M}, 4.04 \mathrm{mmol})$, EtOH $(2 \mathrm{~mL})$ and toluene $(10 \mathrm{~mL})$ were placed under argon. The mixture was heated to $110{ }^{\circ} \mathrm{C}$ and left stirred overnight at this temperature. 
After cooling down, sat. aq. $\mathrm{KHSO}_{4}(5 \mathrm{~mL})$ and water $(5 \mathrm{~mL})$ were added. The organic layer was separated, and the aq. layer was extracted with $\mathrm{CHCl}_{3}(2 \times 10 \mathrm{~mL})$. Combined organic solutions were dried with $\mathrm{Na}_{2} \mathrm{SO}_{4}$ and evaporated. Column chromatography (100 g of $\mathrm{SiO}_{2}, \mathrm{CHCl}_{3} / \mathrm{MeOH}, 7: 1$ ) furnished the title product as a yellow solid (440 $\mathrm{mg}$, 85\%). ${ }^{1} \mathrm{H}$ NMR (300 MHz, $\mathrm{CDCl}_{3}$ ): $\delta=1.27$ (s, $6 \mathrm{H}, 2 \times \mathrm{Me}$ ), 1.43-1.57 (m, $4 \mathrm{H}, 2 \times \mathrm{CH}_{2}$ ), 1.60-1.80 (m, $6 \mathrm{H}, 3 \times \mathrm{CH}_{2}$ ), 1.83 (s, $3 \mathrm{H}, \mathrm{Me}$ ), 3.17-3.27 (m, $2 \mathrm{H}, \mathrm{CH}_{2}$ ), 3.35-3.53 (m, 2 H, $\mathrm{OCH}_{2}$ ), 3.66 (s, $3 \mathrm{H}, \mathrm{OMe}$ ), 3.72-3.87 (m, 2 H, NCH ), 4.54 (br. s, $\left.1 \mathrm{H}\right), 5.02$ (s, $1 \mathrm{H}$ ), 5.98 (s, 1 H, Ar), 7.12 (s, $1 \mathrm{H}, \mathrm{Ar}$ ), 7.17 (d, $J=5.1 \mathrm{~Hz}, 1 \mathrm{H}, \mathrm{Ar}$ ), 8.15 (d, $J=5.1 \mathrm{~Hz}, 1 \mathrm{H}$, Ar) ppm. ${ }^{13} \mathrm{C}$ NMR (125.7 MHz, $\left.\mathrm{CDCl}_{3}\right): \delta=18.7(+)$, 19.7(-), 25.3(-), 25.4(-), 27.4(-), 29.1(+), 30.8(-), 31.9(-), 44.1(-), 55.9(+), 57.2(-), 62.5(-), 67.2(-), 99.0(+), 113.2(-), 115.6(-), 125.20(+), 126.3(+), 126.6(+), 127.2(-), 145.6(-), 146.1(-), 146.5(+), 146.8(-), 157.0(-), 172.9(-) ppm. HRMS (ESI): found 515.2300; calc. for $\mathrm{C}_{28} \mathrm{H}_{35} \mathrm{~N}_{2} \mathrm{O}_{5} \mathrm{Cl}[\mathrm{M}+\mathrm{H}]^{+}$ 515.2307.<smiles>COCCCCN1c2cc(OC)c(-c3ccnc(Cl)c3C(=O)O)cc2C(C)=CC1(C)C</smiles>

4-(1-(4-Acetoxybutyl)-7-methoxy-2,2,4-trimethyl-1,2-dihydroquinolin-6-yl)-2chloronicotinic acid (342-Ac)

Compound 324-Ac was prepared according to the known procedure. ${ }^{[149]}$ In a screw-cap tube a solution of compound 342-THP (207 mg, $0.4 \mathrm{mmol}$ ) in a mixture of AcOH (4 mL) and $\mathrm{AcCl}(4 \mathrm{~mL})$ was placed. This solution was stirred with heating at $80{ }^{\circ} \mathrm{C}$ for $1 \mathrm{~h}$. Afterwards, the reaction mixture was diluted with $\mathrm{H}_{2} \mathrm{O}$, neutralized with sat. aq. $\mathrm{NaHCO}_{3}$ and extracted with $\mathrm{CHCl}_{3}(3 \times 40 \mathrm{~mL})$. Combined organic solutions were dried with $\mathrm{Na}_{2} \mathrm{SO}_{4}$ and evaporated in vacuo. The residue was purified by column chromatography (40 $\mathrm{g}$ of $\mathrm{SiO}_{2}, \mathrm{CHCl}_{3} / \mathrm{MeOH}, 7: 1$ ) to yield $125 \mathrm{mg}$ (66\%) of the title compound as an orange powder. ${ }^{1} \mathrm{H}$ NMR (300 MHz, $\left.\mathrm{CDCl}_{3}\right): \delta=1.26$ (s, $\left.6 \mathrm{H}, 2 \times \mathrm{Me}\right), 1.58-1.72(\mathrm{~m}, 4 \mathrm{H}$, 2× $\mathrm{CH}_{2}$ ), 1.81 (s, $3 \mathrm{H}, \mathrm{Me}$ ), 1.99 (s, $\left.3 \mathrm{H}, \mathrm{Me}\right), 3.15-3.29$ (m, $\left.2 \mathrm{H}, \mathrm{CH}_{2}\right), 3.64$ (s, $3 \mathrm{H}$, OMe), 4.00-4.14 (m, 2 H, CH ), 5.03 (s, 1 H), 5.92 (s, 1 H, Ar), 6.97 (s, 1 H, Ar), 7.21 (d, $J=4.5 \mathrm{~Hz}, 1 \mathrm{H}, \mathrm{Ar}$ ), 8.22 (br. s, $1 \mathrm{H}, \mathrm{Ar}$ ) ppm. HRMS (ESI): found 473.1837; calc. for $\mathrm{C}_{25} \mathrm{H}_{29} \mathrm{~N}_{2} \mathrm{O}_{5} \mathrm{Cl}[\mathrm{M}+\mathrm{H}]^{+} 473.1838$. 
<smiles>CC(=O)OCCCCN1c2cc3oc(=O)c4c(Cl)nccc4c3cc2C(C)=CC1(C)C</smiles>

\section{4-(4-Chloro-9,9,11-trimethyl-5-oxo-6,7,8,9-tetrahydropyrido[4',3’:4,5]pyrano[3,2-}

g]quinolin-8-yl)butyl acetate (343-OAc)

To a solution of compound 342-Ac (10 mg, $0.02 \mathrm{mmol})$ in $\mathrm{CH}_{2} \mathrm{Cl}_{2}(1 \mathrm{~mL}) 2 \mathrm{M}$ solution of $\mathrm{BBr}_{3}(130 \mu \mathrm{L}, 0.13 \mathrm{mmol})$ in $\mathrm{CH}_{2} \mathrm{Cl}_{2}$ was added at $0{ }^{\circ} \mathrm{C}$ under Ar. The resulting mixture was stirred overnight at r.t. Afterwards, the reaction mixture was quenched with sat. aq. $\mathrm{NaHCO}_{3}(5 \mathrm{~mL})$ and extracted with $\mathrm{CH}_{2} \mathrm{Cl}_{2}(3 \times 5 \mathrm{~mL})$. Combined organic extracts were dried with $\mathrm{Na}_{2} \mathrm{SO}_{4}$ and evaporated. Column chromatography (15 g of $\mathrm{SiO}_{2}$, $n$-hexane/EtOAc, 2:1) provided $4 \mathrm{mg}$ (36\%) of the title compound. ${ }^{1} \mathrm{H}$ NMR (600 MHz, $\mathrm{CDCl}_{3}$ ): $\delta=1.37$ (s, $\left.6 \mathrm{H}, 2 \times \mathrm{Me}\right), 1.65-1.75\left(\mathrm{~m}, 4 \mathrm{H}, 2 \times \mathrm{CH}_{2}\right), 2.04$ (d, $J=1.02 \mathrm{~Hz}, 3 \mathrm{H}$, Me), 2.07 (s, 3 H, Me), 3.28 (m, 2 H, $\mathrm{CH}_{2}$ ), 4.12 (t, $J=6.0 \mathrm{~Hz}, 2$ H, $\mathrm{CH}_{2}$ ), 5.32 (m, $1 \mathrm{H}$ ), 6.25 (s, 1 H, Ar), 7.43 (s, 1 H, Ar), 7.61 (d, $J=5.5$ Hz, 1 H, Ar), 8.43 (d, $J=5.5 \mathrm{~Hz}, 1 \mathrm{H}$, Ar) ppm. HRMS (ESI): found 463.1386; calc. for $\mathrm{C}_{24} \mathrm{H}_{25} \mathrm{~N}_{2} \mathrm{O}_{4} \mathrm{Cl}[\mathrm{M}+\mathrm{Na}]^{+} 463.1395$.<smiles>CC(=O)OCCCN1c2cc3oc(=O)c4c(=O)n(C)ccc4c3cc2C(C)=CC1(C)C</smiles>

4-(3,9,9,11-tetramethyl-4,5-dioxo-3,4,6,7,8,9-hexahydropyrido[4',3’'4,5]pyrano[3,2g]quinolin-8-yl)butyl acetate (344)

A solution of compound 343-OAc (15 mg, $0.034 \mathrm{mmol})$ and MeI $(100 \mu \mathrm{L})$ in MeCN (1 $\mathrm{mL}$ ) stirred at $75{ }^{\circ} \mathrm{C}$ for 2 days under Ar. After cooling down, the reaction mixture was directly subjected to column chromatography (15 g of $\left.\mathrm{SiO}_{2}, \mathrm{CH}_{2} \mathrm{Cl}_{2} / \mathrm{MeOH}, 12: 1\right)$ to afford $4 \mathrm{mg}$ (27\%) of the title compound as a yellow powder. ${ }^{1} \mathrm{H}$ NMR (300 $\left.\mathrm{MHz}, \mathrm{CDCl}_{3}\right)$ : $\delta=1.31$ (s, $6 \mathrm{H}, 2 \times \mathrm{Me}$ ), 1.61-1.75 (m, $4 \mathrm{H}, 2 \times \mathrm{CH}_{2}$ ), 2.02 (s, $3 \mathrm{H}, \mathrm{Me}$ ), 2.05 (s, $3 \mathrm{H}, \mathrm{Me}$ ), 3.22-3.32 (m, 2 H, CH 2 ), 3.55 (s, 3 H, NMe), 4.10 (m, 2 H, $\mathrm{OCH}_{2}$ ), 5.30 (s, $1 \mathrm{H}$ ), 6.19 (s, $1 \mathrm{H}, \mathrm{Ar}$ ), 6.69 (d, $J=7.5 \mathrm{~Hz}, 1 \mathrm{H}, \mathrm{Ar}), 7.30$ (s, $1 \mathrm{H}, \mathrm{Ar}$ ), 7.73 (d, $J=7.5 \mathrm{~Hz}, 1 \mathrm{H}, \mathrm{Ar}$ ) ppm. ${ }^{13} \mathrm{C}$ NMR $\left(125.7 \mathrm{MHz}, \mathrm{CDCl}_{3}\right): \delta=18.8(+), 20.9(+), 26.0(-), 29.3(+), 29.6(-)$, 38.0(+), 44.4(-), 58.1(-), 63.7(-), 96.7(+), 99.10(+), 103.8(-), 119.0(+), 120.6(-), 126.2(), 130.1(+), 143.9(+), 149.2(-), 150.1(-), 156.1(-), 161.1(-), 171.3(-) ppm. HRMS (ESI): found 437.2067; calc. for $\mathrm{C}_{25} \mathrm{H}_{28} \mathrm{~N}_{2} \mathrm{O}_{5}[\mathrm{M}+\mathrm{H}]^{+}$437.2071. HPLC: $\mathrm{B} / \mathrm{A}=50 / 50$ to 100/0 in 
25 min, detection at $254 \mathrm{~nm}, t_{\mathrm{R}}=12.2 \mathrm{~min}$ (99 \%). UV-Vis spectral data in $\mathrm{CH}_{2} \mathrm{Cl}_{2}$ : $\lambda_{\text {abs,max }}=422 \mathrm{~nm} ; \varepsilon=11500 \mathrm{M}^{-1} \mathrm{~cm}^{-1}, \lambda_{\text {em,max }}=497 \mathrm{~nm}, \Phi_{\mathrm{fl}}=0.56$ (standard: Lucifer Yellow, $\Phi_{\mathrm{fl}}=0.21$ in $\mathrm{H}_{2} \mathrm{O}$ ). UV-Vis spectral data in $\mathrm{MeOH}: \lambda_{\mathrm{abs}, \max }=427 \mathrm{~nm} ; \varepsilon=$ $18800 \mathrm{M}^{-1} \mathrm{~cm}^{-1}, \lambda_{\mathrm{em}, \max }=524 \mathrm{~nm}, \Phi_{\mathrm{fl}}=0.46$ (standard: Lucifer Yellow, $\Phi_{\mathrm{fl}}=0.21$ in $\left.\mathrm{H}_{2} \mathrm{O}\right)$.<smiles>Cc1c(C=O)c(=O)oc2cc3c(cc12)CCCN3C</smiles>

\section{4,9-Dimethyl-2-oxo-1,2,6,7,8,9-hexahydropyrano[3,2-g]quinoline-3-carbaldehyde} (348)

In a Schlenk flask $\mathrm{POCl}_{3}(675 \mathrm{mg}, 4.4 \mathrm{mmol}$ ) and DMF (642 mg, $8.8 \mathrm{mmol}$ ) were placed under Ar. The resulting solution was warmed up to $50{ }^{\circ} \mathrm{C}$ and stirred for $30 \mathrm{~min}$ at this temperature. Afterwards, a solution of coumarin 347 (100 mg, $0.44 \mathrm{mmol})$ in DMF (2 $\mathrm{mL}$ ) was added dropwise. The reaction mixture was stirred for $10 \mathrm{~min}$, and then poured onto ice. Acidity of the resulting slurry was adjusted with $1 \mathrm{M} \mathrm{NaOH}$ to $\mathrm{pH} 12$, and the mixture was extracted with $\mathrm{CH}_{2} \mathrm{Cl}_{2}(3 \times 20 \mathrm{~mL})$. Combined organic solutions were dried with $\mathrm{Na}_{2} \mathrm{SO}_{4}$ and evaporated. The crude product was subjected to column chromatography (30 g of $\mathrm{SiO}_{2}$, $n$-hexane/EtOAc, 1:1) to furnish $28 \mathrm{mg}$ (25\%) of the title compound as an orange powder. ${ }^{1} \mathrm{H}$ NMR (300 MHz, $\left.\mathrm{CDCl}_{3}\right): \delta=1.98\left(\mathrm{~m}, 2 \mathrm{H}, \mathrm{CH}_{2}\right), 2.74$ 2.80 (m, 2 H, CH ), 2.75 (s, 3 H, Me), 3.01 (s, 3 H, NMe), 3.42 (m, 2 H, NCH ), 6.30 (s, $1 \mathrm{H}, \mathrm{Ar}), 7.29$ (m, $1 \mathrm{H}, \mathrm{Ar}), 10.34$ (s, $1 \mathrm{H}, \mathrm{CHO}) \mathrm{ppm}$.<smiles>CN1CCCc2cc3c(cc21)oc(=O)c1cnccc13</smiles>

\section{8-Methyl-8,9,10,11-tetrahydropyrido[4',3’:4,5]-5H-pyrano[3,2-g]quinolin-5-one} (349)

To a solution of compound 348 (28 mg, $0.11 \mathrm{mmol}$ ) in formamide $(1 \mathrm{~mL})$ conc. $\mathrm{H}_{2} \mathrm{SO}_{4}$ $(1 \mathrm{~mL})$ was added dropwise at $0{ }^{\circ} \mathrm{C}$. The resulting mixture was heated to $90{ }^{\circ} \mathrm{C}$ and left stirred overnight at this temperature. Afterwards, the solid reaction mixture was cooled down to $0{ }^{\circ} \mathrm{C}$, and water $(20 \mathrm{~mL})$ was added. Acidity of the resulting slurry was adjusted with $1 \mathrm{M} \mathrm{NaOH}$ to $\mathrm{pH} 10$, and the mixture was extracted with $\mathrm{CH}_{2} \mathrm{Cl}_{2}(3 \times 30 \mathrm{~mL})$. Combined organic extracts were dried with $\mathrm{Na}_{2} \mathrm{SO}_{4}$ and evaporated. The residue was subjected 
to column chromatography (30 g of $\left.\mathrm{SiO}_{2}, \mathrm{CH}_{2} \mathrm{Cl}_{2} / \mathrm{MeOH}, 20: 1\right)$ to afford $8 \mathrm{mg}$ (27\%) of the title compound. ${ }^{1} \mathrm{H}$ NMR (300 MHz, $\left.\mathrm{CDCl}_{3}\right): \delta=2.00\left(\mathrm{~m}, 2 \mathrm{H}, \mathrm{CH}_{2}\right), 2.82(\mathrm{~m}, 2 \mathrm{H}$, $\mathrm{CH}_{2}$ ), 2.99 (s, $3 \mathrm{H}, \mathrm{NMe}$ ), 3.39 (m, 2 H, NCH ), 6.38 (s, $1 \mathrm{H}, \mathrm{Ar}$ ), 7.47 (m, $1 \mathrm{H}, \mathrm{Ar}$ ), 7.61 (m, $J=5.6 \mathrm{~Hz}, 1 \mathrm{H}, \mathrm{Ar}$ ), 8.71 (d, $J=5.6 \mathrm{~Hz}, 1 \mathrm{H}, \mathrm{Ar}$ ), 9.37 (d, $J=0.8 \mathrm{~Hz}, 1 \mathrm{H}, \mathrm{Ar}$ ) ppm. MS (ESI): m/z (positive mode, rel. int., \%) = $267(100)[\mathrm{M}+\mathrm{H}]^{+}$. UV-Vis spectral data in MeOH: $\lambda_{\text {abs,max }}=377 \mathrm{~nm} ; \varepsilon=14200 \mathrm{M}^{-1} \mathrm{~cm}^{-1}, \lambda_{\text {em,max }}=500 \mathrm{~nm}, \Phi_{\mathrm{fl}}=0.02$ (standard: Coumarin 153, $\Phi_{\mathrm{fl}}=0.69$ in $\left.\mathrm{EtOH}\right)$.<smiles>O=[N+]([O-])c1c(Br)ccc2cnccc12</smiles>

\section{6-Bromo-5-nitroisoquinoline (350)}

A solution of 6-bromoisoquinoline (2080 mg; $10 \mathrm{mmol}$ ) in a $\mathrm{H}_{2} \mathrm{SO}_{4} / \mathrm{HNO}_{3}$ mixture (1:1, $10 \mathrm{~mL}$ ) was stirred for $2 \mathrm{~h}$ at r.t. Afterwards, the reaction mixture was poured onto ice, $\mathrm{pH}$ was adjusted to 9 using 22\% aq. ammonia, and the white precipitate was filtered out, washed and dried. This crude product (2070 mg; 93\%) was used without further purification. ${ }^{1} \mathrm{H}$ NMR (400 MHz, $\left.\mathrm{CDCl}_{3}\right): \delta=7.54$ (d, $\left.J=6.0 \mathrm{~Hz}, 1 \mathrm{H}_{\mathrm{ar}}\right), 7.86$ (d, $J=8.8 \mathrm{~Hz}$, $1 \mathrm{H}_{\mathrm{ar}}$ ), 8.03 (d, $J=8.8 \mathrm{~Hz}, 1 \mathrm{H}_{\mathrm{ar}}$ ), 8.73 (d, $J=6.0 \mathrm{~Hz}, 1 \mathrm{H}_{\mathrm{ar}}$ ), $9.36\left(\mathrm{~s}, 1 \mathrm{H}_{\mathrm{ar}}\right) \mathrm{ppm}$.<smiles>Nc1c(Br)ccc2cnccc12</smiles>

\section{5-Amino-6-bromoisoquinoline (351)}

$\mathrm{NH}_{4} \mathrm{Cl}$ (4066 mg, $76 \mathrm{mmol}$ ) was dissolved in 40\% aq. EtOH $(50 \mathrm{~mL})$. To this solution iron powder (2128 mg, $38 \mathrm{mmol}$ ) was added, and the resulting suspension was stirred for $10 \mathrm{~min}$ at r.t. Afterwards, compound 350 (1690 mg, $7.6 \mathrm{mmol}$ ) was added thereto. The reaction mixture was stirred for $1 \mathrm{~h}$ at r.t., and then filtered through a plug of Celite washing with $\mathrm{EtOH}$. The filtrate was partially evaporated; the precipitated crude product was filtered out using a glass filter and dried. ${ }^{1} \mathrm{H}$ NMR (400 MHz, $\mathrm{CDCl}_{3}$ ): $\delta=4.73$ (br. s, $2 \mathrm{H}, \mathrm{NH}_{2}$ ), 7.28 (d, $J=8.8 \mathrm{~Hz}, 1 \mathrm{H}, \mathrm{Ar}$ ), 7.57 (d, $J=6.0 \mathrm{~Hz}, 1 \mathrm{H}, \mathrm{Ar}$ ), 7.63 (d, $J=8.8 \mathrm{~Hz}$, $1 \mathrm{H}, \mathrm{Ar}$ ), 8.54 (d, $J=5.9 \mathrm{~Hz}, 1 \mathrm{H}, \mathrm{Ar}$ ), 9.16 (s, $1 \mathrm{H}, \mathrm{Ar}$ ) ppm. MS (ESI): m/z (positive mode, rel. int., \%) = $223(100)\left[\mathrm{M}+\mathrm{H},{ }^{79} \mathrm{Br}\right]^{+}, 225(95)\left[\mathrm{M}+\mathrm{H},{ }^{81} \mathrm{Br}\right]^{+}$.<smiles>N#Cc1c(Br)ccc2cnccc12</smiles> 


\section{5-Cyano-6-bromoisoquinoline (352-CN)}

A Schlenk flask was charged with a solution of compound 351 (100 mg, $0.52 \mathrm{mmol})$ in DMSO (3 mL) and CuCN (61 mg, $0.68 \mathrm{mmol})$. The resulting mixture was heated to 50 ${ }^{\circ} \mathrm{C}$ with stirring, and $t \mathrm{BuONO}(206 \mu \mathrm{L}, 1.56 \mathrm{mmol})$ was injected. The reaction mixture was stirred for $2 \mathrm{~h}$ at $50{ }^{\circ} \mathrm{C}$, then cooled down to r.t. and poured in $0.1 \mathrm{M} \mathrm{HCl}(\sim 20 \mathrm{~mL})$. The resulted slurry was extracted with $\mathrm{CH}_{2} \mathrm{Cl}_{2}(4 \times 20 \mathrm{~mL})$. Combined organic solutions were dried with $\mathrm{Na}_{2} \mathrm{SO}_{4}$ and evaporated. The residue was subjected to column chromatography (40 g of $\mathrm{SiO}_{2}, n$-hexane/EtOAc, 1:1) to afford $13 \mathrm{mg}$ (11\%) of a yellowish crystalline solid. ${ }^{1} \mathrm{H}$ NMR (400 MHz, $\mathrm{CDCl}_{3}$ ): $\delta=7.86$ (d, $J=8.8 \mathrm{~Hz}, 1 \mathrm{H}, \mathrm{Ar}$ ), 7.99 (dt, $J=5.9$ and $0.9 \mathrm{~Hz}, 1 \mathrm{H}, \mathrm{Ar}$ ), 8.08 (dd, $J=8.8$ and $0.8 \mathrm{~Hz}, 1 \mathrm{H}, \mathrm{Ar}$ ), 8.77 (d, $J=5.9 \mathrm{~Hz}, 1$ H, Ar), 9.34 (d, $J=0.9 \mathrm{~Hz}, 1 \mathrm{H}, \mathrm{Ar}$ ) ppm. ${ }^{13} \mathrm{C}$ NMR (100 MHz, $\left.\mathrm{CDCl}_{3}\right): \delta=112.4,115.1$, 117.4, 126.2, 131.2, 131.5, 133.3, 136.8, 146.4, 152.8 ppm. MS (ESI): m/z (positive mode, rel. int., \%) = $233(100)\left[\mathrm{M}+\mathrm{H},{ }^{79} \mathrm{Br}\right]^{+}, 235(96)\left[\mathrm{M}+\mathrm{H},{ }^{81} \mathrm{Br}\right]^{+}$.<smiles>COc1cc2c(cc1-c1ccc3cnccc3c1C#N)C(C)=CC(C)(C)N2CCCC[OH2+]</smiles>

6-(7-Methoxy-2,2,4-trimethyl-1-(4-(tetrahydro-2H-pyran-2-yloxy)butyl)-1,2dihydroquinolin-6-yl)isoquinoline-5-carbonitrile (353)

In a screw-cap tube, compound 338 (105 mg, $0.215 \mathrm{mmol}$ ), compound 352-CN (50 mg, $0.215 \mathrm{mmol}), \mathrm{Pd}\left(\mathrm{PPh}_{3}\right)_{4}(12.5 \mathrm{mg}, 0.011 \mathrm{mmol}, 5 \mathrm{~mol} \%)$, sat. aq. solution of $\mathrm{Na}_{2} \mathrm{CO}_{3}$ (430 $\mu \mathrm{L}, 2 \mathrm{M}, 0.86 \mathrm{mmol}$ ) and toluene ( $3 \mathrm{~mL}$ ) were placed under argon. The mixture was heated to $110{ }^{\circ} \mathrm{C}$ and left stirred overnight at this temperature. After cooling, the reaction mixture was filtered through a plug of Celite eluting with $\mathrm{CH}_{2} \mathrm{Cl}_{2}$, and the filtrate was evaporated. Column chromatography (30 g of $\mathrm{SiO}_{2}$, cyclohexane/EtOAc, 1:2) furnished the title product as an orange oil (86 mg, 78\%). ${ }^{1} \mathrm{H}$ NMR (400 MHz, $\left.\mathrm{CDCl}_{3}\right): \delta=1.24$ (s, $6 \mathrm{H}, 2 \times \mathrm{Me}$ ), $1.47-1.60$ (m, $\left.4 \mathrm{H}, 2 \times \mathrm{CH}_{2}\right), 1.67-1.85$ (m, $\left.6 \mathrm{H}, 3 \times \mathrm{CH}_{2}\right), 1.97$ (s, $3 \mathrm{H}, \mathrm{Me}$ ), 3.30-3.37 (m, 2 H, CH 2 ), 3.43-3.53 (m, 2 H, $\mathrm{CH}_{2}$ ), 3.80-3.90 (m, $2 \mathrm{H}, \mathrm{CH}_{2}$ ), 3.85 (s, 3 H, OMe), 4.59 (m, 1 H), 5.15 (s, 1 H), 6.12 (s, 1 H, Ar), 7.10 (s, 1 H, Ar), 7.77 (d, J = 8.6 Hz, $1 \mathrm{H}, \mathrm{Ar}$ ), 8.06 (d, $J=5.9 \mathrm{~Hz}, 1 \mathrm{H}, \mathrm{Ar}$ ), 8.11 (d, $J=8.8 \mathrm{~Hz}, 1 \mathrm{H}, \mathrm{Ar}$ ), 8.67 (d, $J=5.9$ Hz, 1 H, Ar), 9.27 (s, 1 H, Ar) ppm. MS (ESI): m/z (positive mode, rel. int., \%) = 512 (100) $[\mathrm{M}+\mathrm{H}]^{+}$. 
<smiles>CC1=CC(C)(C)N(CCCCBr)c2cc3oc(=O)c4c5ccncc5ccc4c3cc21</smiles>

\section{8-(4-Bromobutyl)-9,9,11-trimethyl-5-oxo-5,6,8,9-tetrahydroiso-}

\section{quinolino[6',5':4,5]pyrano[3,2-g]quinoline (354)}

In a screw-cap tube a solution of compound 353 (10 mg, $0.019 \mathrm{mmol})$ in conc. aq. $\mathrm{HBr}$ $(1 \mathrm{~mL})$ was stirred for $3 \mathrm{~h}$ at $130{ }^{\circ} \mathrm{C}$. After cooling, the reaction mixture was diluted with water $(\sim 5 \mathrm{~mL})$, quenched with $\mathrm{NaHCO}_{3}$ and extracted with $\mathrm{CH}_{2} \mathrm{Cl}_{2}(3 \times 10 \mathrm{~mL})$. Combined organic solutions were dried with $\mathrm{Na}_{2} \mathrm{SO}_{4}$ and evaporated. The crude product was purified by column chromatography (15 g of $\mathrm{SiO}_{2}$, cyclohexane/EtOAc, 1:2) to furnish 6 $\operatorname{mg}(66 \%)$ of the title compound as a red solid. ${ }^{1} \mathrm{H}$ NMR (400 MHz, $\left.\mathrm{CDCl}_{3}\right): \delta=1.43$ (s, 6 H, 2×Me), 1.80-1.90 (m, 2 H, $\mathrm{CH}_{2}$ ), 1.95-2.04 (m, 2 H, $\mathrm{CH}_{2}$ ), 2.12 (s, $3 \mathrm{H}, \mathrm{Me}$ ), 3.313.38 (m, 2 H, $\mathrm{CH}_{2}$ ), 3.49 (t, J = 6.6 Hz, 2 H, CH 2 ), 5.38 (s, $\left.1 \mathrm{H}\right), 6.38$ (s, $1 \mathrm{H}, \mathrm{Ar}$ ), 7.66 (s, $1 \mathrm{H}, \mathrm{Ar}$ ), 8.19 (d, $J=8.9 \mathrm{~Hz}, 1 \mathrm{H}, \mathrm{Ar}), 8.25$ (d, $J=8.9 \mathrm{~Hz}, 1 \mathrm{H}, \mathrm{Ar}$ ), 8.67 (d, $J=6.3 \mathrm{~Hz}, 1$ H, Ar), 9.25 (s, $1 \mathrm{H}, \mathrm{Ar}$ ), 9.58 (d, $J=6.2 \mathrm{~Hz}, 1 \mathrm{H}, \mathrm{Ar}$ ) ppm. MS (ESI): m/z (positive mode, rel. int., \%) = $477(91)\left[\mathrm{M}+\mathrm{H},{ }^{79} \mathrm{Br}\right]^{+}, 479(100)\left[\mathrm{M}+\mathrm{H},{ }^{81} \mathrm{Br}\right]^{+}$.<smiles>CN(C)c1ccc2c(c1)oc(=O)c1c3cccnc3ccc21</smiles>

\section{8- $N, N$-dimethylamino-5H-chromeno[3,4-f]quinolin-5-one (356)}

The synthesis was carried out analogously to known procedure. ${ }^{[152]} \mathrm{LiCl}$ (12 mg, 0.275 mmol) was placed in a Schlenk flask. This flask was evacuated and dried with a heatgun. After cooling, the flask was flushed with Ar, and compound 358 (20 mg, $0.055 \mathrm{mmol}$ ), 3-bromopyridine-2-carbaldehyde (10 mg, $0.055 \mathrm{mmol}$ ), $\mathrm{CuCl}$ (23 mg, $0.231 \mathrm{mmol}$ ), $\mathrm{Pd}\left(\mathrm{PPh}_{3}\right)_{4}$ (6.3 mg, $\left.0.005 \mathrm{mmol}, 10 \mathrm{~mol} \%\right)$ and DMSO $(1 \mathrm{~mL})$ were added. The resulting mixture was stirred for $15 \mathrm{~h}$ at $90{ }^{\circ} \mathrm{C}$. Afterwards, the reaction mixture was left to cool down, diluted with $\mathrm{CH}_{2} \mathrm{Cl}_{2}$ and filtered through a plug of $\mathrm{SiO}_{2}$ eluting with $\mathrm{CH}_{2} \mathrm{Cl}_{2}$. The filtrate was washed with brine, dried with $\mathrm{Na}_{2} \mathrm{SO}_{4}$ and evaporated. Purification of the crude product by column chromatography (35 g of $\mathrm{SiO}_{2}, n$-hexane/EtOAc, 1:1) gave 7 mg (44\%) of the title product. ${ }^{1} \mathrm{H}$ NMR (400 MHz, $\left.\mathrm{CDCl}_{3}\right): \delta=3.06$ (s, $6 \mathrm{H}, \mathrm{NMe}_{2}$ ), 6.57 (d, $J=2.6 \mathrm{~Hz}, 1 \mathrm{H}, \mathrm{Ar}$ ), 6.71 (dd, $J=9.0$ and $2.6 \mathrm{~Hz}, 1 \mathrm{H}, \mathrm{Ar}$ ), 7.60 (dd, $J=8.7$ and 3.9 Hz, 1 H, Ar), 7.94 (d, $J=9.2$ Hz, 1 H, Ar), 8.26 (d, $J=9.2$ Hz, 1 H, Ar), 8.37 (d, $J=9.1$ 
Hz, $1 \mathrm{H}$, Ar), 8.91 (br. s, $1 \mathrm{H}, \mathrm{Ar}$ ), 10.04 (dd, $J=8.8$ and $1.1 \mathrm{~Hz}, 1 \mathrm{H}, \mathrm{Ar}$ ) ppm. UV-Vis spectral data in $\mathrm{MeOH}: \lambda_{\mathrm{abs}, \max }=411 \mathrm{~nm} ; \varepsilon=16700 \mathrm{M}^{-1} \mathrm{~cm}^{-1}, \lambda_{\mathrm{em}, \max }=577 \mathrm{~nm}, \Phi_{\mathrm{fl}}=$ 0.11 (standard: Coumarin 153, $\Phi_{\mathrm{fl}}=0.54$ in EtOH).<smiles>CNc1ccc2c(C)c(B3OC(C)(C)C(C)(C)O3)c(=O)oc2c1</smiles>

7-N,N-Dimethylamino-4-methyl-3-(4,4,5,5-tetramethyl-1,3,2-dioxaborolan-2yl)coumarin (357)

A Schlenk flask was charged with dried AcOK (103 mg, $1.05 \mathrm{mmol}$ ), compound 355-Br (100 mg, $0.35 \mathrm{mmol}$ ), bis(pinacolato)diboron (108 mg, $0.42 \mathrm{mmol}$ ), $\mathrm{PdCl}_{2}(\mathrm{dppf})$ (15.4 mg, $0.02 \mathrm{mmol}, 6 \mathrm{~mol} \%)$ and dioxane (3 mL). The resulting mixture was stirred overnight at $80{ }^{\circ} \mathrm{C}$. After cooling, water $(\sim 10 \mathrm{~mL})$ and $\mathrm{CH}_{2} \mathrm{Cl}_{2}(\sim 10 \mathrm{~mL})$ were added, the organic layer was separated, and the aq. phase was extracted with $\mathrm{CH}_{2} \mathrm{Cl}_{2}(3 \times 20 \mathrm{~mL})$. Combined organic solutions were dried with $\mathrm{Na}_{2} \mathrm{SO}_{4}$ and evaporated. The crude product was purified by column chromatography (40 g of $\mathrm{SiO}_{2}, n$-hexane/EtOAc, 2:1) to yield $40 \mathrm{mg}$ (35\%) of the title compound. ${ }^{1} \mathrm{H}$ NMR (400 MHz, $\mathrm{CDCl}_{3}$ ): $\delta=1.39$ (s, $12 \mathrm{H}, 4 \times \mathrm{Me}$ ), 2.46 (s, $3 \mathrm{H}, \mathrm{Me}$ ), 3.04 (s, $6 \mathrm{H}, 2 \mathrm{Me}$ ), 6.50 (d, $J=2.6 \mathrm{~Hz}, 1 \mathrm{H}, \mathrm{Ar}$ ), 6.60 (dd, $J=9.0$ and 2.6 $\mathrm{Hz}, 1 \mathrm{H}, \mathrm{Ar}), 7.44$ (d, $J=9.0 \mathrm{~Hz}, 1 \mathrm{H}, \mathrm{Ar}) \mathrm{ppm} .{ }^{13} \mathrm{C}$ NMR $\left(100 \mathrm{MHz}, \mathrm{CDCl}_{3}\right): \delta=$ 18.1(+), 24.8(+), 40.2(+), 84.0(-), 98.2(+), 108.6(+), 125.7(+), 152.8(-), 155.9(-), 158.9(-), 163.2(-) ppm. MS (ESI): m/z (positive mode, rel. int., \%) = $330(42)[\mathrm{M}+\mathrm{H}]^{+}$, $352(38)[\mathrm{M}+\mathrm{Na}]^{+}, 681(100)[2 \mathrm{M}+\mathrm{Na}]^{+}$.<smiles>CCCSc1c(C)c2ccc(C)cc2oc1=O</smiles>

\section{7- $N, N$-Dimethylamino-4-methyl-3-trimethylstannylcoumarin (358)}

A screw-cap tube was charged with coumarin 355- $\mathrm{Br}$ (100 mg, $0.35 \mathrm{mmol}$ ), hexamethylditin (151 mg, $0.46 \mathrm{mmol}$ ), $\mathrm{Pd}\left(\mathrm{PPh}_{3}\right)_{4}(20 \mathrm{mg}, 0.017 \mathrm{mmol}, 5 \mathrm{~mol} \%$ ) and toluene (2 mL). The resulting mixture was stirred overnight at $120^{\circ} \mathrm{C}$ under Ar. After cooling, the reaction mixture was filtered through a pad of Celite eluting with $\mathrm{CH}_{2} \mathrm{Cl}_{2}$ ). The filtrate was evaporated, and the residue was subjected to column chromatography (30 g of $\mathrm{SiO}_{2}, n$ hexane/EtOAc, 2:1) to afford $63 \mathrm{mg}$ (49\%) of the title compound. ${ }^{1} \mathrm{H}$ NMR (300 MHz, $\mathrm{CDCl}_{3}$ ): $\delta=0.35$ (s, $9 \mathrm{H}, \mathrm{SnMe}_{3}$ ), 2.38 (s, $3 \mathrm{H}, \mathrm{Me}$ ), 3.02 (s, $6 \mathrm{H}, \mathrm{NMe}_{2}$ ), 6.46 (d, $J=2.6$ 
Hz, $1 \mathrm{H}, \mathrm{Ar}$ ), 6.55 (dd, $J=8.9$ and $2.6 \mathrm{~Hz}, 1 \mathrm{H}, \mathrm{Ar}$ ), 7.39 (d, $J=8.9 \mathrm{~Hz}, 1 \mathrm{H}, \mathrm{Ar}$ ) ppm. ${ }^{13} \mathrm{C} \mathrm{NMR}\left(125.7 \mathrm{MHz}, \mathrm{CDCl}_{3}\right): \delta=-6.6(+), 20.3(+), 40.0(+), 98.1(+), 108.3(+)$, 110.7(+), 122.8(-), 125.1(+), 152.6(-), 156.0(-), 160.2(-), 165.3(-) ppm. HRMS (ESI): found 368.0663; calc. for $\mathrm{C}_{15} \mathrm{H}_{21} \mathrm{NO}_{2} \mathrm{Sn}[\mathrm{M}+\mathrm{H}]^{+} 368.0669$.<smiles></smiles>

\section{1-Chloro-8- $N, N$-dimethylamino-5H-chromeno[3,4-f]isoquinolin-5-one (359)}

Compound 359 was prepared from compound 358 and 2-chloro-4-iodopyridine-3-carbaldehyde using the procedure described for compound 356. Yield: $10 \mathrm{mg}$ (56\%) as an orange powder. ${ }^{1} \mathrm{H}$ NMR (300 MHz, $\mathrm{CDCl}_{3}$ ): $\delta=3.12$ (s, $6 \mathrm{H}, \mathrm{NMe}_{2}$ ), 6.60 (d, $J=2.6$ Hz, $1 \mathrm{H}, \mathrm{Ar}$ ), 6.76 (dd, $J=9.1$ and $2.6 \mathrm{~Hz}, 1 \mathrm{H}, \mathrm{Ar}$ ), 7.97 (d, $J=9.2 \mathrm{~Hz}, 1 \mathrm{H}, \mathrm{Ar}$ ), 8.20 (d, $J=9.3 \mathrm{~Hz}, 1 \mathrm{H}, \mathrm{Ar}$ ), 8.46 (d, $J=6.1 \mathrm{~Hz}, 1 \mathrm{H}, \mathrm{Ar}$ ), 8.65 (dd, $J=9.2$ and $0.9 \mathrm{~Hz}, 1 \mathrm{H}, \mathrm{Ar}$ ), 9.48 (dd, $J=6.1$ and $0.8 \mathrm{~Hz}, 1 \mathrm{H}$, Ar) ppm. MS (ESI): m/z (positive mode, rel. int., \%) = $325(100)\left[\mathrm{M}+\mathrm{H},{ }^{35} \mathrm{Cl}\right]^{+}, 327(30)\left[\mathrm{M}+\mathrm{H},{ }^{37} \mathrm{Cl}\right]^{+}$.<smiles>CNc1ccc2c(c1)oc(=O)c1c2ccc2cccnc21</smiles>

\section{8- $N, N$-Dimethylamino-5H-chromeno[4,3-h]quinolin-5-one (360)}

From boronic ester 357: A screw-cap tube was charged with compound 357 (20 mg, $0.061 \mathrm{mmol})$, 2-bromopyridine-3-carbaldehyde (13.6 mg, $0.073 \mathrm{mmol}), \mathrm{Pd}\left(\mathrm{PPh}_{3}\right)_{4}(3.5$ mg, $0.003 \mathrm{mmol}, 5 \mathrm{~mol} \%), 2 \mathrm{M}$ sat. aq. $\mathrm{NaHCO}_{3}(122 \mu \mathrm{L}, 0.244 \mathrm{mmol})$ and toluene $(1 \mathrm{~mL})$. The resulting mixture was stirred overnight at $120{ }^{\circ} \mathrm{C}$ under Ar. After cooling, the reaction mixture was filtered through a plug of Celite eluting with $\mathrm{CH}_{2} \mathrm{Cl}_{2}$. The filtrate was evaporated, and the residue was subjected to column chromatography (30 g of $\mathrm{SiO}_{2}$, $\mathrm{CH}_{2} \mathrm{Cl}_{2} / \mathrm{MeOH}, 20: 1$ ) to afford $9 \mathrm{mg}$ (51\%) of the title compound. ${ }^{1} \mathrm{H}$ NMR (400 MHz, DMSO-d6): $\delta=3.03$ (s, $6 \mathrm{H}, \mathrm{NMe}_{2}$ ), 6.58 (d, $J=2.5 \mathrm{~Hz}, 1 \mathrm{H}, \mathrm{Ar}$ ), 6.79 (dd, $J=9.0$ and $2.5 \mathrm{~Hz}, 1 \mathrm{H}, \mathrm{Ar}$ ), 7.57 (dd, $J=8.1$ and $4.2 \mathrm{~Hz}, 1 \mathrm{H}, \mathrm{Ar}$ ), 8.19 (d, $J=9.2 \mathrm{~Hz}, 1 \mathrm{H}, \mathrm{Ar}$ ), 8.31 (d, $J=8.9 \mathrm{~Hz}, 1 \mathrm{H}, \mathrm{Ar}$ ), 8.39 (d, $J=8.6 \mathrm{~Hz}, 1 \mathrm{H}, \mathrm{Ar}$ ), 8.40 (d, $J=8.1 \mathrm{~Hz}, 1 \mathrm{H}, \mathrm{Ar}$ ), 9.03 (m, $1 \mathrm{H}, \mathrm{Ar})$ ppm. ${ }^{13} \mathrm{C}$ NMR (100 MHz, DMSO-d6): $\delta=97.9(+), 106.3(-), 109.8(+)$, 111.9(-), 120.8(+), 121.7(+), 125.9(+), 126.9(-), 136.1(+), 136.9(+), 141.0(-), 147.6(-), 
152.3(+), 153.2(-), 154.4(-), 157.2(-) ppm. HRMS (ESI): found 291.1128; calc. for $\mathrm{C}_{18} \mathrm{H}_{14} \mathrm{~N}_{2} \mathrm{O}_{2}[\mathrm{M}+\mathrm{H}]^{+}$291.1128. UV-Vis spectral data in $\mathrm{MeOH}: \lambda_{\text {abs,max }}=420 \mathrm{~nm} ; \varepsilon=$ $13200 \mathrm{M}^{-1} \mathrm{~cm}^{-1}, \lambda_{\mathrm{em}, \max }=560 \mathrm{~nm}, \Phi_{\mathrm{fl}}=0.29$ (standard: Coumarin 153, $\Phi_{\mathrm{fl}}=0.54$ in $\mathrm{EtOH})$.<smiles>Cc1c(-c2ncccc2C=O)c(=O)oc2cc(N(C)C)ccc12</smiles>

2-(7- $N, N$-Dimethylamino-4-methyl-2-oxo-1,2-dihydrochromen-3-yl)nicotinaldehyde (361)

Compound 361 was prepared from compound 358 and 2-bromopyridine-3-carbaldehyde using the procedure described for compound 356. Column chromatography (30 g of $\mathrm{SiO}_{2}$, $\left.\mathrm{CH}_{2} \mathrm{Cl}_{2} / \mathrm{MeOH}, 20: 1\right)$ yielded $14 \mathrm{mg}$ (82\%) of the title compound as a yellow powder. ${ }^{1} \mathrm{H}$ NMR (300 MHz, $\mathrm{CDCl}_{3}$ ): $\delta=2.22$ (s, $3 \mathrm{H}, \mathrm{Me}$ ), 3.07 (s, $6 \mathrm{H}, \mathrm{NMe}_{2}$ ), 6.55 (d, $J=2.5 \mathrm{~Hz}$, $1 \mathrm{H}, \mathrm{Ar}$ ), 6.66 (dd, $J=9.0$ and $2.5 \mathrm{~Hz}, 1 \mathrm{H}, \mathrm{Ar}$ ), 7.42-7.54 (m, $1 \mathrm{H}, \mathrm{Ar}$ ), 7.51 (d, $J=9.0$ Hz, 1 H, Ar), 8.30 (d, J = 7.8 Hz, 1 H, Ar), 8.89 (br. s, 1 H, Ar), 10.01 (s, 1 H, CHO) ppm. HRMS (ESI): calc. for $\mathrm{C}_{18} \mathrm{H}_{16} \mathrm{~N}_{2} \mathrm{O}_{3}[\mathrm{M}+\mathrm{H}]^{+}$309.1234; found 309.1230.<smiles>CN(C)c1ccc2c(c1)oc(=O)c1c3cc[n+](C)cc3ccc21</smiles>

\section{8- $N, N$-dimethylamino-2-methyl-5-oxo-5H-chromeno[3,4-f]isoquinolin-2-ium trifluoroacetate (362)}

In a screw-cap tube, a solution of compound 359 (7 mg, $0.021 \mathrm{mmol})$ and MeI (4.6 $\mathrm{mg}$, $0.032 \mathrm{mmol}$ ) in DMF (2 mL) was stirred overnight at $100^{\circ} \mathrm{C}$. After cooling, the reaction mixture was diluted with water $(\sim 10 \mathrm{~mL})$ and extracted with $\mathrm{CH}_{2} \mathrm{Cl}_{2}(3 \times 20 \mathrm{~mL})$. Combined organic solutions were dried with $\mathrm{Na}_{2} \mathrm{SO}_{4}$ and evaporated in vacuo. The crude product was purified by column chromatography (30 g of $\mathrm{SiO}_{2}, \mathrm{MeCN} / \mathrm{H}_{2} \mathrm{O}, 5: 1+0.1$ $\mathrm{v} / \mathrm{v} \% \mathrm{TFA}$ ) to afford $3 \mathrm{mg}$ (44\%) of the title compound as an orange powder. ${ }^{1} \mathrm{H}$ NMR (300 MHz, DMSO-d6): $\delta=3.11$ (s, 6 H, NMe $), 4.41$ (s, $3 \mathrm{H}, \mathrm{Me}$ ), 6.68 (d, $J=2.5 \mathrm{~Hz}, 1$ H, Ar), 6.90 (dd, $J=9.2$ and $2.6 \mathrm{~Hz}, 1 \mathrm{H}, \mathrm{Ar}$ ), 8.30 (d, $J=9.4 \mathrm{~Hz}, 1 \mathrm{H}, \mathrm{Ar}$ ), 8.57 (d, $J=$ $8.9 \mathrm{~Hz}, 1 \mathrm{H}, \mathrm{Ar}$ ), 8.74 (d, $J=9.3 \mathrm{~Hz}, 1 \mathrm{H}, \mathrm{Ar}$ ), 8.78 (dd, $J=7.2$ and $1.5 \mathrm{~Hz}, 1 \mathrm{H}, \mathrm{Ar}$ ), 9.64 (d, $J=7.2 \mathrm{~Hz}, 1 \mathrm{H}, \mathrm{Ar}$ ), 9.81 (s, $1 \mathrm{H}, \mathrm{Ar}$ ) ppm. MS (ESI): m/z (positive mode, rel. 
int., \%) $=305(100) \mathrm{M}^{+}$. UV-Vis spectral data in MeOH: $\lambda_{\text {abs,max }}=467 \mathrm{~nm} ; \varepsilon=13400 \mathrm{M}^{-}$ ${ }^{1} \mathrm{~cm}^{-1}, \lambda_{\text {em,max }}=608 \mathrm{~nm}, \Phi_{\mathrm{fl}}=0.26$ (standard: Coumarin 153, $\Phi_{\mathrm{fl}}=0.54 \mathrm{in} \mathrm{EtOH}$ ).<smiles>CCOC(=O)CCCN1c2cc3oc(=O)cc(C)c3cc2C(C)=CC1(C)C</smiles>

9-(Ethoxycarbonylpropyl)-8,9-dihydro-4,6,8,8-tetramethyl-2H-pyrano[3,2g]quinolin-2-one (362)

Ethyl acetoacetate (1.50 g, $11.5 \mathrm{mmol}$ ) was added to a solution of phenol 288-H (1.95 g, $6.44 \mathrm{mmol})$ in ethanol $(4 \mathrm{~mL})$ followed by addition of dry $\mathrm{ZnCl}_{2}(1.3 \mathrm{~g}, 9.6 \mathrm{mmol})$. The reaction mixture was heated in an open flask $\left(90^{\circ} \mathrm{C}\right)$ for $20 \mathrm{~h}$. The obtained green-grey slurry was cooled, dissolved in dichloromethane $(50 \mathrm{~mL})$ and shaken with $2 \%$ aq. ammonia solution $(50 \mathrm{~mL})$. The organic layer was separated and passed through a plug of $\mathrm{SiO}_{2}$ eluting with $\mathrm{CH}_{2} \mathrm{Cl}_{2} / \mathrm{Et}_{2} \mathrm{O}$ (1:1). After evaporation of solvents, the oily residue was purified by column chromatography (cyclohexane/ $\mathrm{CH}_{2} \mathrm{Cl}_{2} / \mathrm{Et}_{2} \mathrm{O}, 4: 4: 1$ ) to give $1.52 \mathrm{~g}$ (64\%) of a pale-yellow solid. ${ }^{1} \mathrm{H}$ NMR (400 MHz, $\mathrm{CDCl}_{3}$ ): $\delta=1.28$ (t, $J=7.2,3 \mathrm{H}, \mathrm{Et}$ ), 1.35 (s, $6 \mathrm{H}, 2 \times \mathrm{Me}$ ), 1.90 (m, $2 \mathrm{H}, \mathrm{CH}_{2}$ ), 1.98 (d, $J=0.8 \mathrm{~Hz}, 3 \mathrm{H}, \mathrm{Me}$ ), 2.31 (d, $J=0.8 \mathrm{~Hz}, 3 \mathrm{H}$, Me), 2.38 (m, 2 H, $\mathrm{CH}_{2}$ ), 3.29 (m, 2 H, $\mathrm{NCH}_{2}$ ), 4.16 (q, $J=7.2 \mathrm{~Hz}, 2 \mathrm{H}, \mathrm{Et}$ ), 5.28 (q, $J=$ $0.8 \mathrm{~Hz}, 1 \mathrm{H}$ ), 5.91 (q, $J=0.8 \mathrm{~Hz}, 1 \mathrm{H}$ ), 6.34 (s, $1 \mathrm{H}, \mathrm{Ar}$ ), 7.09 (s, $1 \mathrm{H}, \mathrm{Ar}$ ) ppm. ${ }^{13} \mathrm{C} \mathrm{NMR}$ (100.7 MHz, $\left.\mathrm{CDCl}_{3}\right): \delta=14.2,18.5,18.7,22.7,29.0,31.4,43.8,57.7,60.6,97.2,108.8$, 109.0, 118.8, 119.8, 126.3, 129.8, 147.3, 152.9, 155.8, 162.0, 172.8 ppm. MS (ESI): m/z (positive mode, rel. int., \%) = $761.4(100)[2 \mathrm{M}+\mathrm{Na}]^{+}, 392.3(70)[\mathrm{M}+\mathrm{Na}]^{+}, 370.3(18)$ $[\mathrm{M}+\mathrm{H}]^{+}$.<smiles>CCOC(=O)CCCN1c2cc3oc(=O)cc(C)c3cc2C(C=O)=CC1(C)C</smiles>

8,9-Dihydro-9-ethoxycarbonylpropyl-6-formyl-4,8,8-trimethyl-2H-pyrano[3,2g]quinolin-2-one (363)

Finely powdered $\mathrm{SeO}_{2}$ (676 mg, $\left.6.09 \mathrm{mmol}\right)$ was added to a hot $\left(90^{\circ} \mathrm{C}\right)$ solution of 362 $(1.50 \mathrm{~g}, 4.06 \mathrm{mmol})$ in dioxane $(30 \mathrm{~mL})$. Then the reaction mixture was stirred at $100{ }^{\circ} \mathrm{C}$ for $1 \mathrm{~h}$. After cooling, dioxane was evaporated in vacuo, the residue was diluted with 
dichloromethane $(50 \mathrm{~mL})$ and the organic layer was washed with sat. aq. $\mathrm{NaHCO}_{3}$ and dried with $\mathrm{MgSO}_{4}$. All volatiles were evaporated, and the residue was purified by column chromatography (cyclohexane/ $\mathrm{CH}_{2} \mathrm{Cl}_{2} / \mathrm{Et}_{2} \mathrm{O}, 2: 2: 0.6$ ). Fractions containing the product were collected and, after evaporation of solvents, the residue was triturated with $\mathrm{Et}_{2} \mathrm{O}$ to afford $794 \mathrm{mg}$ (51\%) of a yellow solid. ${ }^{1} \mathrm{H}$ NMR (300 MHz, $\left.\mathrm{CDCl}_{3}\right): \delta=1.28(\mathrm{t}, J=7.2$ Hz, 3 H, Et), 1.50 (s, 6 H, 2×Me), 1.90 (m, 2 H, CH 2 ), 2.37 (d, $J=0.8$ Hz, 3H, Me), 2.39 (m, $2 \mathrm{H}, \mathrm{CH}_{2}$ ), 3.32 (m, $2 \mathrm{H}, \mathrm{NCH}_{2}$ ), 4.17 (q, $J=7.2 \mathrm{~Hz}, 2 \mathrm{H}, \mathrm{Et}$ ), 5.97 (q, $J=0.8 \mathrm{~Hz}, 1$ H), 6.21 (s, $1 \mathrm{H}, \mathrm{Ar}), 6.43$ (s, $1 \mathrm{H}, \mathrm{Ar}), 8.54$ (s, $1 \mathrm{H}, \mathrm{Ar}), 9.58$ (s, $1 \mathrm{H}, \mathrm{CHO}) \mathrm{ppm} .{ }^{13} \mathrm{C}$ NMR (125.7 MHz, $\left.\mathrm{CDCl}_{3}\right): \delta=14.3(+), 18.6(+), 22.9(-), 27.9(+), 31.3(-), 43.9(-)$, 58.0(-), 60.7(-), 98.3(+), 109.7(+), 109.9(-), 113.4(-), 122.3(+), 130.8(-), 146.9(-), 152.0(+), 153.4(-), 156.0(-), 161.6(-), 172.6(-), 191.8(+) ppm. MS (ESI): m/z (positive mode, rel. int., \%) = $761.4(100)[2 \mathrm{M}+\mathrm{Na}]^{+}, 392.3(70)[\mathrm{M}+\mathrm{Na}]^{+}, 370.3(18)[\mathrm{M}+\mathrm{H}]^{+}$.<smiles>CCOC(=O)CCCN1c2cc3oc(=O)c(Br)c(C)c3cc2C(C=O)=CC1(C)C</smiles>

Ethyl (3-bromo-6-formyl-8,9-dihydro-4,8,8-trimethyl-2H-pyrano[3,2-g]quinolin-2one)-9-butanoate (364)

A solution of bromine (184 mg, $1.15 \mathrm{mmol})$ in $\mathrm{AcOH}(1 \mathrm{~mL})$ was added to a solution of 363 (421 mg, $1.09 \mathrm{mg}$ ) in AcOH (8 mL). The reaction mixture was stirred at room temperature for $10 \mathrm{~min}$ and left in refrigerator $\left(5^{\circ} \mathrm{C}\right)$ for $1 \mathrm{~h}$. The precipitate was filtered, washed with cold ether and dried. Afterwards, this crude product was dissolved in dichloromethane, washed with sat. aq. $\mathrm{NaHCO}_{3}$ and passed through a plug of $\mathrm{SiO}_{2}$ eluting with $\mathrm{CH}_{2} \mathrm{Cl}_{2} / \mathrm{Et}_{2} \mathrm{O}$ (1:1). After evaporation of solvents, the title compound was precipitated from $\mathrm{Et}_{2} \mathrm{O}$ with $n$-hexane; yield $530 \mathrm{mg}(96 \%)$ as a solvate with $\mathrm{CH}_{2} \mathrm{Cl}_{2}$ according to ${ }^{1} \mathrm{H}$ NMR (364 $1 / 2 \mathrm{CH}_{2} \mathrm{Cl}_{2}$ ). ${ }^{1} \mathrm{H}$ NMR (300 MHz, $\left.\mathrm{CDCl}_{3}\right): \delta=1.28$ (t, $J=7.2 \mathrm{~Hz}, 3 \mathrm{H}$, Et), 1.50 (s, $6 \mathrm{H}, 2 \times \mathrm{Me}$ ), 1.90 (m, $2 \mathrm{H}, \mathrm{CH}_{2}$ ), 2.40 (m, 2 H, $\mathrm{CH}_{2}$ ), 2.55 (s, $3 \mathrm{H}, \mathrm{Me}$ ), 3.32 (m, $2 \mathrm{H}, \mathrm{NCH}_{2}$ ), 4.17 (q, J= $\left.7.2 \mathrm{~Hz}, 2 \mathrm{H}, \mathrm{Et}\right), 5.27\left(1 \mathrm{H}, 1 / 2 \cdot \mathrm{CH}_{2} \mathrm{Cl}_{2}\right.$ ), 6.23 (s, $1 \mathrm{H}$ ), 6.44 (s, $1 \mathrm{H}, \mathrm{Ar}), 8.62$ (s, $1 \mathrm{H}, \mathrm{Ar}), 9.49$ (s, $1 \mathrm{H}, \mathrm{CHO}) \mathrm{ppm} .{ }^{13} \mathrm{C} \mathrm{NMR}\left(125.7 \mathrm{MHz}, \mathrm{CDCl}_{3}\right): \delta=$ 14.3(+), 19.4(+), 22.8(-), 28.1(+), 31.2(-), 44.0(-), 58.2(-), 60.8(-), 98.0(+), 106.7(-), 109.8(-), 113.8(-), 122.8(+), 130.5(-), 147.0(-), 151.9(-), 152.3(+), 154.4(-), 157.6(-), 172.6(-), 191.7(+) ppm. HRMS (ESI, $\mathrm{C}_{22} \mathrm{H}_{24} \mathrm{NO}_{5} \mathrm{Br}$ ): 462.0754/460.0752 (found 
$[\mathrm{M}-\mathrm{H}]^{-}$), 462.0746/460.0765 (calc.); HRMS (ESI, $\mathrm{C}_{22} \mathrm{H}_{24} \mathrm{NO}_{5} \mathrm{Br}$ ): 486.0697/484.0725 (found $[\mathrm{M}+\mathrm{Na}]^{-}$), 486.0711/484.0730 (calc.); 464.0888/462.0897 (found $[\mathrm{M}+\mathrm{H}]^{-}$), 464.0891/464.0911 (calc.).<smiles>CCOC(=O)CCCN1c2cc3oc(=O)c(S)c(C)c3cc2C(C=O)=CC1(C)C</smiles>

Ethyl 4-(6-formyl-4,8,8-trimethyl-2-oxo-3-(trimethylstannyl)-1,2,8,9-tetrahydro pyrano[3,2-g]quinolin-9-yl)butanoate (368)

A screw-cap tube was charged with coumarin 364 (631 mg, $1.36 \mathrm{mmol}$ ), hexamethylditin (538 mg, $1.64 \mathrm{mmol}), \mathrm{Pd}\left(\mathrm{PPh}_{3}\right)_{4}(161 \mathrm{mg}, 0.14 \mathrm{mmol}, 10 \mathrm{~mol} \%)$ and toluene (10 mL). The resulting mixture was stirred overnight at $120^{\circ} \mathrm{C}$ under Ar. After cooling, the reaction mixture was filtered through a pad of Celite eluting with $\mathrm{CH}_{2} \mathrm{Cl}_{2}$. The filtrate was evaporated, and the residue was subjected to column chromatography (30 g of $\mathrm{SiO}_{2}, n$ hexane/EtOAc, 2:1) to afford $364 \mathrm{mg}$ (49\%) of the title compound. ${ }^{1} \mathrm{H}$ NMR (400 MHz, $\mathrm{CDCl}_{3}$ ): $\delta=0.37$ (s, $9 \mathrm{H}, \mathrm{SnMe}_{3}$ ), 1.30 (t, $\left.J=7.1 \mathrm{~Hz}, 3 \mathrm{H}, \mathrm{Et}\right), 1.50$ (s, $6 \mathrm{H}, 2 \times \mathrm{Me}$ ), 1.59 (s, 3 H, Me), 1.88-1.97 (m, 2 H, $\mathrm{CH}_{2}$ ), 2.38-2.45 (m, 2 H, $\mathrm{CH}_{2}$ ), 2.44 (s, $3 \mathrm{H}, \mathrm{Me}$ ), 3.303.36 (m, 2 H, NCH $), 4.18$ (q, J = 7.1 Hz, 2 H, Et), 6.21 (s, 1 H), 6.41 (s, 1 H, Ar), 7.26 (s, $1 \mathrm{H}, \mathrm{Ar}$ ), 8.58 (s, $1 \mathrm{H}, \mathrm{Ar}$ ), 9.60 (s, $1 \mathrm{H}, \mathrm{CHO}$ ) ppm. HRMS (ESI): calc. for $\mathrm{C}_{25} \mathrm{H}_{33} \mathrm{NO}_{5} \mathrm{Sn}[\mathrm{M}+\mathrm{H}]^{+}$548.1458; found 548.1446.



\section{2-Bromo-4-(1,3-dioxolan-2-yl)pyridine (369)}

A mixture of 2-bromopyridine-3-carbaldehyde (500 mg, $2.69 \mathrm{mmol}$ ), ethylene glycol $(0.6 \mathrm{~mL}), \mathrm{TsOH} \cdot \mathrm{H}_{2} \mathrm{O}(204 \mathrm{mg}, 1.08 \mathrm{mmol})$ and toluene $(50 \mathrm{~mL})$ was refluxed with a Dean-Stark trap for $5 \mathrm{~h}$. After cooling, the reaction mixture was diluted with EtOAc (50 $\mathrm{mL})$, washed with water $(50 \mathrm{~mL})$, aq. $\mathrm{NaHCO}_{3}(50 \mathrm{~mL})$, and again with water $(50 \mathrm{~mL})$. The organic layer was dried with $\mathrm{MgSO}_{4}$ and evaporated to give $577 \mathrm{mg}$ (93\%) of the crude product which was used without further purification. ${ }^{1} \mathrm{H}$ NMR $\left(400 \mathrm{MHz}, \mathrm{CDCl}_{3}\right)$ : $\delta=4.06-4.19\left(\mathrm{~m}, 4 \mathrm{H}, 2 \times \mathrm{CH}_{2}\right.$ ), 6.04 (s, $1 \mathrm{H}, \mathrm{Ar}$ ), 7.31 (dd, $J=7.6$ and $4.7 \mathrm{~Hz}, 1 \mathrm{H}, \mathrm{Ar}$ ), 7.89 (dd, $J=7.6$ and $2.0 \mathrm{~Hz}, 1 \mathrm{H}, \mathrm{Ar}$ ), 8.37 (dd, $J=4.7$ and $2.0 \mathrm{~Hz}, 1 \mathrm{H}, \mathrm{Ar}$ ) ppm. 
<smiles>CCOC(=O)CCCn1c(=O)c2c(ccc3cccnc32)c2cc3c(cc21)N(CC)C(C)(C)C=C3C=O</smiles>

Ethyl 4-(11-formyl-9,9-dimethyl-5,6,8,9-tetrahydroquinolino[7',8':4,5]pyrano[3,2g]quinolin-8-yl)butanoate (370)

To a solution of compound 371 (30 mg, $0.06 \mathrm{mmol}$ ) in $\mathrm{CH}_{2} \mathrm{Cl}_{2} / \mathrm{MeOH}(1: 2,3 \mathrm{~mL}$ ) $\mathrm{Cs}_{2} \mathrm{CO}_{3}$ (4 mg, $0.012 \mathrm{mmol}$ ) was added, and the resulting mixture left stirred for $2.5 \mathrm{~h}$ at r.t. Afterwards, water $(15 \mathrm{~mL})$ was added, and the resulting slurry was extracted with $\mathrm{CH}_{2} \mathrm{Cl}_{2}(3 \times 15 \mathrm{~mL})$. Combined organic solutions were dried with $\mathrm{Na}_{2} \mathrm{SO}_{4}$ and evaporated. The crude product was purified by column chromatography (30 g of $\mathrm{SiO}_{2}$, $\left.\mathrm{CH}_{2} \mathrm{Cl}_{2} / \mathrm{MeOH}, 20: 1\right)$ to provide $20 \mathrm{mg}$ (71\%) of the title product. ${ }^{1} \mathrm{H}$ NMR (400 MHz, $\mathrm{CDCl}_{3}$ ): $\delta=1.29$ (t, $\left.J=7.1 \mathrm{~Hz}, 3 \mathrm{H}, \mathrm{Et}\right), 1.54$ (s, $6 \mathrm{H}, 2 \times \mathrm{Me}$ ), 1.91-2.01 (m, $2 \mathrm{H}, \mathrm{CH}_{2}$ ), 2.45 (t, $J=6.9 \mathrm{~Hz}, 2 \mathrm{H}, \mathrm{CH}_{2}$ ), 3.33-3.40 (m, $2 \mathrm{H}, \mathrm{NCH}_{2}$ ), 4.18 (q, $J=7.1 \mathrm{~Hz}, 2 \mathrm{H}, \mathrm{Et}$ ), 6.26 (s, 1 H), 6.53 (s, 1 H, Ar), 7.48 (dd, $J=8.1$ and $4.3 \mathrm{~Hz}, 1 \mathrm{H}, \mathrm{Ar}$ ), 8.10 (d, $J=8.9 \mathrm{~Hz}$, $1 \mathrm{H}, \mathrm{Ar}$ ), 8.18 (dd, $J=8.2$ and $1.9 \mathrm{~Hz}, 1 \mathrm{H}, \mathrm{Ar}$ ), 8.20 (d, $J=9.0 \mathrm{~Hz}, 1 \mathrm{H}, \mathrm{Ar}$ ), 9.16 (s, 1 H, Ar), 9.24 (dd, $J=4.3$ and $1.9 \mathrm{~Hz}, 1 \mathrm{H}, \mathrm{Ar}$ ), 9.64 (s, $1 \mathrm{H}, \mathrm{CHO}) \mathrm{ppm} .{ }^{13} \mathrm{C}$ NMR (100 $\left.\mathrm{MHz}, \mathrm{CDCl}_{3}\right): \delta=14.3(+), 22.8(-), 28.0(+), 31.0(-), 43.9(-), 58.1(-), 60.8(-), 98.4(+)$, 107.0(-), 112.8(-), 113.7(-), 120.3(+), 121.1(+), 121.6(+), 127.1(-), 130.8(-), 135.3(+), 136.5(+), 140.8(-), 146.9(-), 147.9(-), 152.5(+), 154.9(-), 158.5(-), 173.3(-), 192.2(+) ppm. HRMS (ESI): calc. for $\mathrm{C}_{28} \mathrm{H}_{26} \mathrm{~N}_{2} \mathrm{O}_{5}[\mathrm{M}+\mathrm{H}]^{+} 471.1914$; found 471.1897 .<smiles>CCOC(=O)CCCN1c2cc3oc(=O)c(-c4ncccc4C=O)c(C)c3cc2C(C=O)=CC1(C)C</smiles>

Ethyl 4-(6-formyl-3-(3-formylpyridin-2-yl)-4,8,8-trimethyl-2-oxo-1,2,8,9-tetrahydropyrano[3,2-g]quinolin-9-yl)butanoate (371)

Compound 371 was prepared from compound 368 and 2-bromopyridine-3-carbaldehyde using the procedure described for compound 356. Column chromatography (40 g of $\mathrm{SiO}_{2}$, $\mathrm{CH}_{2} \mathrm{Cl}_{2} / \mathrm{MeOH}$, 30:1) yielded $44 \mathrm{mg}$ (91\%) of the title compound as a yellow powder. ${ }^{1} \mathrm{H}$ NMR (400 MHz, $\mathrm{CDCl}_{3}$ ): $\delta=1.30$ (t, $J=7.1 \mathrm{~Hz}, 3 \mathrm{H}, \mathrm{Et}$ ), 1.55 (s, $6 \mathrm{H}, 2 \times \mathrm{Me}$ ), 1.90$2.01\left(\mathrm{~m}, 2 \mathrm{H}, \mathrm{CH}_{2}\right), 2.26$ (s, $\left.3 \mathrm{H}, \mathrm{Me}\right), 2.43\left(\mathrm{t}, J=6.8 \mathrm{~Hz}, 2 \mathrm{H}, \mathrm{CH}_{2}\right), 3.35-3.42(\mathrm{~m}, 2 \mathrm{H}$, 
$\mathrm{NCH}_{2}$ ), 4.19 (q, J = 7.1 Hz, $\left.2 \mathrm{H}, \mathrm{Et}\right), 6.26$ (s, $\left.1 \mathrm{H}, \mathrm{Ar}\right), 6.52$ (s, $1 \mathrm{H}, \mathrm{Ar}$ ), 7.42-7.49 (m, 1 H, Ar), 7.51-7.57 (m, 1 H, Ar), 7.63-7.70 (m, 1 H, Ar), 8.73 (s, 1 H, Ar), 9.61 (s, 1 H, CHO), 10.02 (s, $1 \mathrm{H}, \mathrm{CHO}$ ) ppm. ${ }^{13} \mathrm{C}$ NMR (100 MHz, $\left.\mathrm{CDCl}_{3}\right): \delta=14.2(+), 16.3(+)$, 22.8(-), 28.1(+), 31.2(-), 44.0(-), 58.3(-), 60.7(-), 98.1(+), 109.9(-), 113.8(-), 117.9(-), 123.2(+), 123.4(+), 130.6(-), 131.4(-), 136.1(+), 147.5(-), 152.2(+), 152.6(-), 153.9(+), 155.9(-), 156.6(-), 161.2(-), 172.7(-), 190.2(+), 191.9(+) ppm. HRMS (ESI): calc. for $\mathrm{C}_{28} \mathrm{H}_{28} \mathrm{~N}_{2} \mathrm{O}_{6}[\mathrm{M}+\mathrm{H}]^{+}$489.2020; found 489.2019.<smiles>CCOC(=O)CCCN1c2cc3c(cc2C(C=O)=CC1(C)C)C(c1ncccc1C1OCCO1)=C(C)C3</smiles>

Ethyl 4-(3-(3-(1,3-dioxolan-2-yl)pyridin-2-yl)-6-formyl-4,8,8-trimethyl-2-oxo-1,2,8,9tetrahydropyrano[3,2-g]quinolin-9-yl)butanoate (372)

Compound 372 was prepared from compound 368 and compound 369 using the procedure described for compound 356. Column chromatography (35 g of $\mathrm{SiO}_{2}$, $\left.\mathrm{CH}_{2} \mathrm{Cl}_{2} / \mathrm{MeOH}, 40: 1\right)$ yielded $77 \mathrm{mg}$ (80\%) of the title compound as a yellow powder. ${ }^{1} \mathrm{H}$ NMR (400 MHz, $\mathrm{CDCl}_{3}$ ): $\delta=1.30$ (t, $\left.J=7.1 \mathrm{~Hz}, 3 \mathrm{H}, \mathrm{Et}\right), 1.53$ (s, $3 \mathrm{H}, \mathrm{Me}$ ), 1.54 (s, $3 \mathrm{H}$, Me), 1.90-2.00 (m, 2 H, CH 2 ), 2.16 (s, 3 H, Me), 2.42 (t, $J=6.8$ Hz, 2 H, $\mathrm{CH}_{2}$ ), 3.313.41 (m, 2 H, NCH ), 3.83-3.90 (m, 1 H), 3.90-3.99 (m, 2 H), 4.04-4.09 (m, 1 H), 4.19 (q, $J=7.1 \mathrm{~Hz}, 2 \mathrm{H}, \mathrm{Et}), 5.74$ (s, $1 \mathrm{H}), 6.24$ (s, $1 \mathrm{H}$ ), 6.49 (s, $1 \mathrm{H}, \mathrm{Ar}), 7.42-7.49$ (m, $1 \mathrm{H}$, Ar), 7.51-7.57 (m, 1 H, Ar), 7.63-7.70 (m, 1 H, Ar), 8.68 (s, 1 H, Ar), 9.60 (s, 1 H, CHO) ppm. HRMS (ESI): calc. for $\mathrm{C}_{30} \mathrm{H}_{32} \mathrm{~N}_{2} \mathrm{O}_{7}[\mathrm{M}+\mathrm{H}]^{+}$533.2282; found 533.2279.

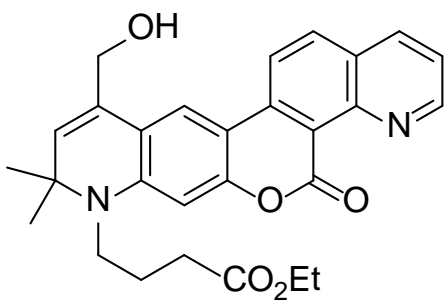

Ethyl

4-(11-hydroxymethyl-9,9-dimethyl-5,6,8,9-tetrahydro-

\section{quinolino[7',8':4,5]pyrano[3,2-g]quinolin-8-yl)butanoate (373)}

From aldehyde 370: To a solution of compound 370 (32 mg, $0.068 \mathrm{mmol}$ ) in THF/EtOH (3:1, $4 \mathrm{~mL}) \mathrm{NaBH}_{4}(3 \mathrm{mg}, 0.068 \mathrm{mmol})$ was added at $0{ }^{\circ} \mathrm{C}$ with stirring. After $5 \mathrm{~min}, 3$ drops of $70 \%$ aq. $\mathrm{HClO}_{4}$ and $\mathrm{Bu}_{4} \mathrm{NIO}_{4}(4.3 \mathrm{mg}, 0.01 \mathrm{mmol})$ were added. After 5 min stirring, the reaction mixture was neutralized with sat. aq. $\mathrm{NaHCO}_{3}$. The resulting mixture 
was diluted with water $(10 \mathrm{~mL})$ and extracted with $\mathrm{CH}_{2} \mathrm{Cl}_{2}(3 \times 10 \mathrm{~mL})$. Combined organic solutions were dried with $\mathrm{Na}_{2} \mathrm{SO}_{4}$ and evaporated in vacuo. The residue was subjected to column chromatography (40 g of $\mathrm{SiO}_{2}, \mathrm{CH}_{2} \mathrm{Cl}_{2} / \mathrm{MeOH}, 8: 1$ ) to furnish $10 \mathrm{mg}$ (31\%) of the title product.

From aldehyde 374: To a solution of compound 374 (30 mg, $0.061 \mathrm{mmol})$ in EtOH (1 $\mathrm{mL}$ ) $\mathrm{Cs}_{2} \mathrm{CO}_{3}$ (2 $\mathrm{mg}, 0.006 \mathrm{mmol}, 10 \mathrm{~mol} \%$ ) was added. The resulting mixture was stirred for $1.5 \mathrm{~h}$ at r.t. Afterwards, sat. aq. $\mathrm{NH}_{4} \mathrm{Cl}(5 \mathrm{~mL})$ and water $(5 \mathrm{~mL})$ were added, and the reaction mixture was extracted with $\mathrm{CH}_{2} \mathrm{Cl}_{2}(3 \times 10 \mathrm{~mL})$. Combined organic solutions were dried with $\mathrm{Na}_{2} \mathrm{SO}_{4}$ and evaporated. The residue was subjected to column chromatography (30 g of $\mathrm{SiO}_{2}, \mathrm{CH}_{2} \mathrm{Cl}_{2} / \mathrm{MeOH}, 20: 1$ ) to give $24 \mathrm{mg}(83 \%)$ of the title product. ${ }^{1} \mathrm{H}$ NMR (400 MHz, $\mathrm{CDCl}_{3}$ ): $\delta=1.30$ (t, $\left.J=6.8 \mathrm{~Hz}, 3 \mathrm{H}, \mathrm{Et}\right), 1.41$ (s, $6 \mathrm{H}, 2 \times \mathrm{Me}$ ), 1.882.00 (m, 2 H, $\mathrm{CH}_{2}$ ), 2.37-2.46 (m, 2 H, $\mathrm{CH}_{2}$ ), 3.28-3.38 (m, $2 \mathrm{H}, \mathrm{NCH}_{2}$ ), 4.18 (q, $J=6.8$ Hz, 2 H, Et), 4.59 (s, 2 H, OCH 2 ), 5.57 (s, 1 H), 6.42 (s, 1 H, Ar), 7.40-7.49 (m, 1 H, Ar), 7.86 (s, $1 \mathrm{H}, \mathrm{Ar}$ ), 8.2 (d, $J=8.5 \mathrm{~Hz}, 1 \mathrm{H}, \mathrm{Ar}$ ), 8.12 (d, $J=8.1 \mathrm{~Hz}, 1 \mathrm{H}, \mathrm{Ar}$ ), 9.19 (s, $1 \mathrm{H}$, Ar) ppm. MS (ESI): m/z (positive mode, rel. int., \%) = $473(100)[\mathrm{M}+\mathrm{H}]^{+}$. UV-Vis spectral data in MeOH: $\lambda_{\text {abs,max }}=438 \mathrm{~nm} ; \varepsilon=19300 \mathrm{M}^{-1} \mathrm{~cm}^{-1}, \lambda_{\mathrm{em}, \max }=574 \mathrm{~nm}, \Phi_{\mathrm{fl}}=$ 0.27 (standard: Coumarin 153, $\Phi_{\mathrm{fl}}=0.54$ in EtOH).<smiles>CCOC(=O)CCCn1c(-c2ncccc2C=O)c(C)c2cc3c(cc21)N(CC)C(C)(C)C=C3CO</smiles>

Ethyl 4-(3-(3-formylpyridin-2-yl)-6-hydroxymethyl-4,8,8-trimethyl-2-oxo-1,2,8,9tetrahydropyrano[3,2-g]quinolin-9-yl)butanoate (374)

To a solution of compound 375 (46 $\mathrm{mg}, 0.086 \mathrm{mmol})$ acetone/water (4:1, $3 \mathrm{~mL})$ $\mathrm{Ts} \mathrm{OH} \cdot \mathrm{H}_{2} \mathrm{O}$ (16 mg, $\left.0.086 \mathrm{mmol}\right)$ was added. The resulting reaction mixture was stirred overnight at reflux. After cooling, sat. aq. $\mathrm{NaHCO}_{3}(4 \mathrm{~mL})$ and brine $(5 \mathrm{~mL})$ were added, and the reaction mixture was extracted with $\mathrm{CH}_{2} \mathrm{Cl}_{2}(3 \times 30 \mathrm{~mL})$. Combined organic solutions were dried with $\mathrm{Na}_{2} \mathrm{SO}_{4}$ and evaporated. The crude product was purified by column chromatography (30 g of $\left.\mathrm{SiO}_{2}, \mathrm{CH}_{2} \mathrm{Cl}_{2} / \mathrm{MeOH}, 20: 1 \rightarrow 10: 1\right)$ to give $30 \mathrm{mg}(71 \%)$ of the title compound. ${ }^{1} \mathrm{H}$ NMR (400 MHz, $\mathrm{CDCl}_{3}$ ): $\delta=1.29$ (t, $J=7.1 \mathrm{~Hz}, 3 \mathrm{H}, \mathrm{Et}$ ), 1.40 (s, 3 H, Me), 1.41 (s, 3 H, Me), 1.89-1.99 (m, 2 H, $\mathrm{CH}_{2}$ ), 2.19 (s, $\left.3 \mathrm{H}, \mathrm{Me}\right), 2.41$ (t, J = 6.9 Hz, $2 \mathrm{H}, \mathrm{CH}_{2}$ ), 3.30-3.37 (m, $2 \mathrm{H}, \mathrm{NCH}_{2}$ ), 4.18 (q, J = 7.1 Hz, $2 \mathrm{H}, \mathrm{Et}$ ), 4.46 (s, $2 \mathrm{H}, \mathrm{OCH}_{2}$ ), 5.55 (s, $1 \mathrm{H}$ ), 6.43 (s, $1 \mathrm{H}, \mathrm{Ar}), 7.36$ (s, $1 \mathrm{H}, \mathrm{Ar}$ ), 7.48 (dd, $J=7.9$ and $4.8 \mathrm{~Hz}, 1 \mathrm{H}, \mathrm{Ar}$ ), 
8.30 (dd, $J=7.9$ and $1.8 \mathrm{~Hz}, 1 \mathrm{H}, \mathrm{Ar}$ ), 8.88 (dd, $J=4.8$ and $1.8 \mathrm{~Hz}, 1 \mathrm{H}, \mathrm{Ar}$ ), 9.98 (s, $1 \mathrm{H}$, CHO) ppm. ${ }^{13} \mathrm{C}$ NMR (100 MHz, $\left.\mathrm{CDCl}_{3}\right): \delta=14.3(+), 16.2(+)$, 22.7(-), 29.0(+), 31.3(-), 44.0(-), 57.8(-), 60.7(-), 62.8(-), 97.3(+), 109.1(-), 116.9(-), 117.6(-), 119.6(+), 123.4(+), 129.8(-), 129.9(+), 131.5(-), 136.1(+), 148.0(-), 152.2(-), 153.7(+), 155.6(-), 156.8(-), 161.4(-), 172.8(-), 190.3(+) ppm. HRMS (ESI): calc. for $\mathrm{C}_{28} \mathrm{H}_{30} \mathrm{~N}_{2} \mathrm{O}_{6}[\mathrm{M}+\mathrm{H}]^{+}$ 491.2177; found 491.2181.<smiles>CCOC(=O)CCCn1c(-c2ncccc2C2OCCO2)c(C)c2cc3c(cc21)N(CC)C(C)(C)C=C3CO</smiles>

Ethyl 4-(3-(3-(1,3-dioxolan-2-yl)pyridin-2-yl)-6-hydroxymethyl-4,8,8-trimethyl-2oxo-1,2,8,9-tetrahydropyrano[3,2-g]quinolin-9-yl)butanoate (375)

To a solution of compound 372 (77 mg, $0.145 \mathrm{mmol})$ in THF/EtOH (3:1, $4 \mathrm{~mL}) \mathrm{NaBH}_{4}$ (5.5 mg, $0.145 \mathrm{mmol}$ ) was added at $0{ }^{\circ} \mathrm{C}$ with stirring. After $5 \mathrm{~min}$, the reaction mixture was quenched with sat. aq. $\mathrm{NH}_{4} \mathrm{Cl}$, diluted with water $(2 \mathrm{~mL})$, and extracted with $\mathrm{CH}_{2} \mathrm{Cl}_{2}$ $(3 \times 10 \mathrm{~mL})$. Combined organic extracts were dried with $\mathrm{Na}_{2} \mathrm{SO}_{4}$ and evaporated. The residue was subjected to column chromatography (30 g of $\mathrm{SiO}_{2}, \mathrm{CH}_{2} \mathrm{Cl}_{2} / \mathrm{MeOH}, 20: 1$ ) to yield $62 \mathrm{mg}(80 \%)$ of the title compound as a yellow solid. ${ }^{1} \mathrm{H}$ NMR (400 $\left.\mathrm{MHz}, \mathrm{CDCl}_{3}\right)$ : $\delta=1.29(\mathrm{t}, J=1.29 \mathrm{~Hz}, 3 \mathrm{H}, \mathrm{Et}), 1.39(\mathrm{~s}, 6 \mathrm{H}, 2 \times \mathrm{Me}), 1.88-1.98\left(\mathrm{~m}, 2 \mathrm{H}, \mathrm{CH}_{2}\right), 2.04$ (s, 3 H, Me), 2.41 (t, $J=6.9 \mathrm{~Hz}, 2 \mathrm{H}, \mathrm{CH}_{2}$ ), 3.28-3.36 (m, $2 \mathrm{H}, \mathrm{NCH}_{2}$ ), 3.81-3.89 (m, $1 \mathrm{H}$, $\mathrm{OCH}_{2}$ ), 3.89-3.97 (m, $2 \mathrm{H}, \mathrm{OCH}_{2}$ ), 4.02-4.10 (m, $1 \mathrm{H}, \mathrm{OCH}_{2}$ ), 4.18 (q, J= 7.1 Hz, $2 \mathrm{H}$, Et), 4.36-4.40 (m, $\left.1 \mathrm{H}, \mathrm{OCH}_{2}\right), 4.41-4.46$ (m, $\left.1 \mathrm{H}, \mathrm{OCH}_{2}\right), 5.56$ (s, $\left.1 \mathrm{H}\right), 5.71$ (s, $\left.1 \mathrm{H}\right)$, 6.39 (s, $1 \mathrm{H}, \mathrm{Ar}$ ), 7.23 (s, $1 \mathrm{H}, \mathrm{Ar}$ ), 7.38 (dd, $J=7.9$ and $4.9 \mathrm{~Hz}, 1 \mathrm{H}, \mathrm{Ar}$ ), 8.04 (dd, $J=$ 7.9 and $1.6 \mathrm{~Hz}, 1 \mathrm{H}, \mathrm{Ar}), 8.69$ (dd, $J=4.9$ and $1.7 \mathrm{~Hz}, 1 \mathrm{H}, \mathrm{Ar}) \mathrm{ppm} .{ }^{13} \mathrm{C}$ NMR (100 $\left.\mathrm{MHz}, \mathrm{CDCl}_{3}\right): \delta=14.3(+), 16.1(+), 22.7(-), 28.9(+), 29.0\left(^{+}\right), 31.4(-), 43.9(-), 57.7(-)$, 60.7(-), 62.4(-), 65.3(-), 65.4(-), 97.3(+), 100.7(+), 109.1(-), 110.0(+), 117.4(-), 119.2(+), 123.1(+), 129.2(+), 129.8(-), 134.1(-), 135.2(+), 147.4(-), 149.7(+), 151.2(-), 153.4(-), 155.5(-), 160.8(-), 172.8(-) ppm. HRMS (ESI): calc. for $\mathrm{C}_{30} \mathrm{H}_{34} \mathrm{~N}_{2} \mathrm{O}_{7}[\mathrm{M}+\mathrm{H}]^{+}$ 535.2439; found 535.2433. 
<smiles>CCOC(=O)CCCN1c2cc3c(cc2C(COC(=O)OCC)=CC1(C)C)-c1ccc2cccnc2c1C3=O</smiles>

Ethyl 4-(11-(di-tert-butoxyphosphoryloxy)methyl-9,9-dimethyl-5,6,8,9-tetrahydroquinolino[7',8':4,5]pyrano[3,2-g]quinolin-8-yl)butanoate (376)

To a stirred and preheated $\left(40{ }^{\circ} \mathrm{C}\right)$ solution of compound $373(10 \mathrm{mg}, 0.021 \mathrm{mmol})$ in $\mathrm{CH}_{2} \mathrm{Cl}_{2}(1 \mathrm{~mL})$ di-t-butyl $N, N$-diisopropylphosphoramidite $(18 \mathrm{mg}, 0.063 \mathrm{mmol}$ ) and $1 H$-tetrazole ( $4.7 \mathrm{mg}, 0.063 \mathrm{mmol}$ ) were added in two equal portions at interval of $20 \mathrm{~min}$ under argon. After further $20 \mathrm{~min}$, the reaction mixture was cooled with dry ice bath $\left(-78{ }^{\circ} \mathrm{C}\right.$ ), and solution of $\mathrm{mCPBA}(15.5 \mathrm{mg}, 70 \%$ purity, $0.063 \mathrm{mmol})$ in $\mathrm{CH}_{2} \mathrm{Cl}_{2}(1 \mathrm{~mL})$ was injected. After $1 \mathrm{~h}$ stirring at $-78{ }^{\circ} \mathrm{C}$, the reaction mixture was allowed to warm to 0 ${ }^{\circ} \mathrm{C}$, and aqueous solutions of $\mathrm{Na}_{2} \mathrm{SO}_{3}(2 \mathrm{~mL}, 10 \%)$ and $\mathrm{NaHCO}_{3}(1 \mathrm{~mL}$, saturated) were added, and the reaction mixture was allowed to warm up to r.t. The organic layer was separated and the aqueous phase was extracted with $\mathrm{CH}_{2} \mathrm{Cl}_{2}(3 \times 10 \mathrm{~mL})$. The combined organic extracts were dried, the solvents were evaporated, and the titled compound was isolated by column chromatography (30 g of $\mathrm{SiO}_{2}, \mathrm{CH}_{2} \mathrm{Cl}_{2} / \mathrm{MeOH}, 15: 1$ ) as a red solid (10 mg; 71\%). ${ }^{1} \mathrm{H}$ NMR (300 MHz, $\mathrm{CDCl}_{3}$ ): $\delta=1.29$ (t, $J=7.1 \mathrm{~Hz}, 3 \mathrm{H}, \mathrm{Et}$ ), 1.43 (s, $6 \mathrm{H}$, $2 \times \mathrm{Me}), 1.48$ (d, $\left.J_{\mathrm{CP}}=1.5 \mathrm{~Hz}, 18 \mathrm{H}, 2 \times t \mathrm{Bu}\right), 1.95\left(\mathrm{~m}, 2 \mathrm{H}, \mathrm{CH}_{2}\right), 2.42(\mathrm{t}, J=6.9 \mathrm{~Hz}, 2 \mathrm{H}$, $\mathrm{CH}_{2}$ ), 3.29-3.38 (m, $2 \mathrm{H}, \mathrm{NCH}_{2}$ ), 4.18 (q, J=7.1 Hz, $2 \mathrm{H}, \mathrm{Et}$ ), 4.85 (d, $J_{\mathrm{CP}}=6.2 \mathrm{~Hz}, 2 \mathrm{H}$, $\mathrm{OCH}_{2}$ ), 5.61 (s, $1 \mathrm{H}$ ), 6.47 (s, $1 \mathrm{H}, \mathrm{Ar}$ ), 7.49 (dd, $J=8.1$ and $4.4 \mathrm{~Hz}, 1 \mathrm{H}, \mathrm{Ar}$ ), 7.96 (s, 1 H, Ar), 8.09 (d, $J=9.0 \mathrm{~Hz}, 1 \mathrm{H}, \mathrm{Ar}$ ), 8.18 (dd, $J=8.2$ and $1.9 \mathrm{~Hz}, 1 \mathrm{H}, \mathrm{Ar}$ ), 8.31 (d, $J=$ $9.1 \mathrm{~Hz}, 1 \mathrm{H}, \mathrm{Ar}$ ), 9.27 (dd, $J=4.4$ and $1.9 \mathrm{~Hz}, 1 \mathrm{H}, \mathrm{Ar}) \mathrm{ppm} .{ }^{13} \mathrm{C}$ NMR (125.7 MHz, $\left.\mathrm{CDCl}_{3}\right): \delta=14.2(+), 29.8\left(+, \mathrm{d}, J_{\mathrm{CP}}=4.3 \mathrm{~Hz}\right), 31.3(-), 43.9(-), 57.7(-), 60.7(-), 66.5(-$, $\left.J_{\mathrm{CP}}=5.6 \mathrm{~Hz}\right), 82.7(-), 82.8(-), 97.7(+), 106.4(-), 117.2(-), 119.3(+), 120.7(+), 121.1\left(^{+}\right)$, 127.0(-), 132.7(+), 135.3(+), 136.8(+), 141.2(-), 147.4(-), 152.3(+), 154.8(-), 158.9(-), 173.0(-) ppm. HRMS (ESI): found 665.2984; calc. for $\mathrm{C}_{36} \mathrm{H}_{45} \mathrm{~N}_{2} \mathrm{O}_{8} \mathrm{P}[\mathrm{M}+\mathrm{H}]^{+}$665.2986.<smiles>CC(C)(C)OOCC1=CC(C)(C)N(CCCC(=O)O)c2cc3oc(=O)c4c(ccc5cccnc54)c3cc21</smiles> 


\section{(11-(Di-tert-butoxyphosphoryloxy)methyl-9,9-dimethyl-5,6,8,9-tetrahydro-}

\section{quinolino[7',8':4,5]pyrano[3,2-g]quinolin-8-yl)butanoic acid (377)}

To a solution of compound 376 (10 mg, $0.015 \mathrm{mmol})$ in $\mathrm{MeOH} / \mathrm{H}_{2} \mathrm{O}(1: 1,2 \mathrm{~mL}) 1 \mathrm{M}$ $\mathrm{NaOH}$ (150 $\mu L, 0.15 \mathrm{mmol})$ was added. The resulting mixture was stirred for $3 \mathrm{~h}$ at r.t.,

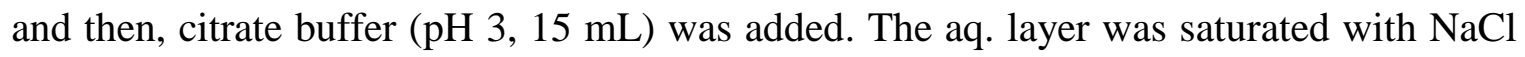
and extracted with $\mathrm{CH}_{2} \mathrm{Cl}_{2}(3 \times 15 \mathrm{~mL})$. Combined organic extracts were dried with $\mathrm{Na}_{2} \mathrm{SO}_{4}$ and evaporated. The residue was subjected with column chromatography (15 g of $\left.\mathrm{SiO}_{2}, \mathrm{CH}_{2} \mathrm{Cl}_{2} / \mathrm{MeOH}, 10: 1 \rightarrow 5: 1\right)$ to afford $7 \mathrm{mg}$ (73\%) of the title compound as a red solid. ${ }^{1} \mathrm{H}$ NMR (400 MHz, $\mathrm{CDCl}_{3}$ ): $\delta=1.41$ (s, $6 \mathrm{H}, 2 \times \mathrm{Me}$ ), 1.50 (br. s, $18 \mathrm{H}, 2 \times t \mathrm{Bu}$ ), 1.91-2.02 (m, 2 H, $\mathrm{CH}_{2}$ ), 2.43-2.52 (m, 2 H, $\mathrm{CH}_{2}$ ), 3.27-3.37 (m, $2 \mathrm{H}, \mathrm{NCH}_{2}$ ), 4.88 (br. s, 2 H, $\mathrm{OCH}_{2}$ ), 5.62 (s, $1 \mathrm{H}$ ), 6.55 (s, $\left.1 \mathrm{H}, \mathrm{Ar}\right), 7.50-7.60$ (m, $\left.1 \mathrm{H}, \mathrm{Ar}\right), 7.90$ (s, $1 \mathrm{H}, \mathrm{Ar}$ ), 8.08-8.16 (m, 1 H, Ar), 8.21-8.35 (m, 2 H, Ar), 9.24-9.34 (m, 1 H, Ar) ppm. MS (ESI): $\mathrm{m} / \mathrm{z}$ (positive mode, rel. int., \%) $=637(100)[\mathrm{M}+\mathrm{H}]^{+}$.<smiles>CC1(C)C=C(COP(O)O[OH2+])c2cc3c(cc2N1CCCC(=O)O)oc(=O)c1c3ccc2cccnc21</smiles>

\section{(9,9-Dimethyl-11-phosphonooxymethyl-5,6,8,9-tetrahydro-}

\section{quinolino[7’,8':4,5]pyrano[3,2-g]quinolin-8-yl)butanoic acid (378)}

To a cooled $\left(0^{\circ} \mathrm{C}\right)$ solution of compound 377 (7 mg, $\left.0.011 \mathrm{mmol}\right)$ in $\mathrm{CH}_{2} \mathrm{Cl}_{2}(1 \mathrm{~mL}) \mathrm{TFA}$ $(100 \mu \mathrm{L})$ was added. The resulting solution was stirred for $40 \mathrm{~min}$ at $0{ }^{\circ} \mathrm{C}$, and then, all volatiles were evaporated in vacuo. The crude product was dissolved in water and freezedried. Yield: $6 \mathrm{mg}$ (quantitative). ${ }^{1} \mathrm{H}$ NMR (300 MHz, $\mathrm{CD}_{3} \mathrm{OD}$ ): $\delta=1.51$ (s, $6 \mathrm{H}, 2 \times \mathrm{Me}$ ), 1.89-2.02 (m, 2 H, CH 2 ), 2.51 (t, $J=6.5$ Hz, 2 H, CH 2 ), 3.46-3.50 (m, 2 H, NCH 2 ), 4.854.89 (m, 2 H, $\mathrm{OCH}_{2}$ ), 5.84 (s, $\left.1 \mathrm{H}\right), 6.77$ (s, $1 \mathrm{H}, \mathrm{Ar}$ ), 8.04-8.11 (m, $2 \mathrm{H}, \mathrm{Ar}$ ), 8.47 (d, $J=$ $9.1 \mathrm{~Hz}, 1 \mathrm{H}, \mathrm{Ar}), 8.63$ (d, $J=9.1 \mathrm{~Hz}, 1 \mathrm{H}, \mathrm{Ar}), 9.08-9.18$ (m, $2 \mathrm{H}, \mathrm{Ar}) \mathrm{ppm} .{ }^{13} \mathrm{C} \mathrm{NMR}$ (125.7 MHz, $\left.\mathrm{CD}_{3} \mathrm{OD}\right): \delta=23.8(-), 29.2(+), 31.4(-)$, 45.3(-), 60.1(-), 67.3(-,d, $J=4.6$ Hz), 98.9(-), 104.3(-), 106.6(-), 115.9(-), 118.2(-), 119.9(-), 121.4(+), 123.1(+), 125.6(+), 129.5(-), 135.4(+), 136.8(+), 140.5(-), 145.6(+), 146.1(-), 148.3(+), 151.5(-), 157.0(-), 176.9(-) ppm. HRMS (ESI): found 525.1417; calc. for $\mathrm{C}_{26} \mathrm{H}_{25} \mathrm{~N}_{2} \mathrm{O}_{8} \mathrm{P}[\mathrm{M}+\mathrm{H}]^{+}$ 513.2748; found 523.1274; calc. for $\mathrm{C}_{26} \mathrm{H}_{25} \mathrm{~N}_{2} \mathrm{O}_{8} \mathrm{P}[\mathrm{M}-\mathrm{H}]^{-}$523.1276. UV-Vis spectral 
data in PBS 7.4: $\lambda_{\mathrm{abs}, \max }=453 \mathrm{~nm} ; \varepsilon=7740 \mathrm{M}^{-1} \mathrm{~cm}^{-1}, \lambda_{\mathrm{em}, \max }=617 \mathrm{~nm}, \Phi_{\mathrm{fl}}=0.44$ (standard: DCM, $\Phi_{\mathrm{fl}}=0.44$ in $\mathrm{EtOH}$ ).<smiles>COc1ccc(C(=O)c2ccc([N+](=O)[O-])cc2)c(Br)c1</smiles>

\section{(2-Bromo-4-methoxyphenyl)-(4-nitrophenyl)methanone (400)}

A dry $50 \mathrm{~mL}$ Schlenk flask was charged with $\mathrm{AlCl}_{3}$ (1.73 g, $\left.13 \mathrm{mmol}\right)$ and $\mathrm{CH}_{2} \mathrm{Cl}_{2}(13$ $\mathrm{mL}$ ). To this suspension, 3-bromoanisole 399 (2.5 g, $13 \mathrm{mmol}$ ) was added under vigorous stirring at $0{ }^{\circ} \mathrm{C}$. After warming up to r.t., a solution of $p$-nitrobenzoyl chloride (2.73 g, 15 mmol) in $\mathrm{CH}_{2} \mathrm{Cl}_{2}(20 \mathrm{~mL})$ was introduced, and the reaction mixture refluxed for $3 \mathrm{~h}$. After cooling down to r.t., the reaction mixture was poured into a mixture of ice and $1 \mathrm{M}$ $\mathrm{HCl}$. The organic layer was separated, the aqueous phase was extracted with $\mathrm{CH}_{2} \mathrm{Cl}_{2}(2 \times$ $100 \mathrm{~mL})$, and the combined organic solutions were washed with $1 \mathrm{M} \mathrm{NaOH}(3 \times 50 \mathrm{~mL})$ and dried with $\mathrm{Na}_{2} \mathrm{SO}_{4}$. Evaporation of volatile solvents in vacuo gave a residue which was recrystallized from ethanol to yield $3.05 \mathrm{~g}$ (71\%) of the title compound as a yellowish powder. ${ }^{1} \mathrm{H}$ NMR (300 MHz, $\mathrm{CDCl}_{3}$ ): $\delta=3.90$ (s, $3 \mathrm{H}, \mathrm{OMe}$ ), 6.97 (dd, $J=8.6$ and 2.4 $\mathrm{Hz}, 1 \mathrm{H}, \mathrm{Ar}$ ), 7.21 (d, $J=2.4 \mathrm{~Hz}, 1 \mathrm{H}, \mathrm{Ar}), 7.39$ (d, $J=8.6,1 \mathrm{H}, \mathrm{Ar}$ ), 7.93 (m, $2 \mathrm{H}, \mathrm{Ar}$ ), 8.30 (m, $2 \mathrm{H}, \mathrm{Ar})$ ppm. ${ }^{13} \mathrm{C}$ NMR (100.7 MHz, $\left.\mathrm{CDCl}_{3}\right): \delta=55.8(+), 113.4(+), 119.1(+)$, 121.5(-), 123.7(+), 130.8(+), 131.2(-), 131.7(+), 142.0(-), 150.3(-), 162.1(-), 193.8(-) ppm. HRMS (ESI): found 335.9863; calc for $\mathrm{C}_{14} \mathrm{H}_{10} \mathrm{NO}_{4} \mathrm{Br}[\mathrm{M}+\mathrm{H}]^{+} 335.9866$.<smiles>COc1ccc(C(=O)c2ccc(N)cc2)c(Br)c1</smiles>

\section{(4-Aminophenyl)(2-bromo-4-methoxyphenyl)methanone (401)}

To $1.0 \mathrm{~g}$ (3.0 mmol) of compound 400 in a mixture of 1,2-dimethoxyethane and ethanol (9 and $11 \mathrm{~mL}$, respectively), a solution of $\mathrm{SnCl}_{2} \cdot 2 \mathrm{H}_{2} \mathrm{O}(2.64 \mathrm{~g}, 11.7 \mathrm{mmol}$ ) in conc. aq $\mathrm{HCl}(20 \mathrm{~mL})$ was added at such a rate that the temperature did not exceed $30^{\circ} \mathrm{C}$. After stirring overnight at r.t., the reaction mixture was poured into an excess of ice-water and $\mathrm{CH}_{2} \mathrm{Cl}_{2}$. Then the aqueous layer was neutralized to $\mathrm{pH} 12$ with solid $\mathrm{NaOH}$, the organic layer was separated, and the aqueous solution was extracted with $\mathrm{CH}_{2} \mathrm{Cl}_{2}(50 \mathrm{~mL})$. The combined organic solutions were dried with $\mathrm{Na}_{2} \mathrm{SO}_{4}$ and evaporated in vacuo to give a residue which was purified by column chromatography (100 g SiO $2, \mathrm{CH}_{2} \mathrm{Cl}_{2} / \mathrm{MeOH}$, 20:1) to furnish $758 \mathrm{mg}$ (82\%) of the title compound. ${ }^{1} \mathrm{H}$ NMR (300 $\left.\mathrm{MHz}, \mathrm{CDCl}_{3}\right): \delta=$ 
3.85 (s, 3 H, OMe), 6.62 (m, 2 H, Ar), 6.89 (dd, $J=8.5$ and $2.4 \mathrm{~Hz}, 1 \mathrm{H}, \mathrm{Ar}$ ), 7.15 (d, $J=$ $2.4 \mathrm{~Hz}, 1 \mathrm{H}, \mathrm{Ar}$ ), 7.39 (d, $J=8.5 \mathrm{~Hz}, 1 \mathrm{H}, \mathrm{Ar}), 7.64$ (m, $2 \mathrm{H}, \mathrm{Ar}) \mathrm{ppm} .{ }^{13} \mathrm{C}$ NMR (100.7 $\left.\mathrm{MHz}, \mathrm{CDCl}_{3}\right): \delta=55.6(+), 113.0(+), 113.9(+), 118.4(+), 120.7(-), 127.2(-), 130.3(+)$, 132.9(+), 133.6(-), 151.2(-), 160.7(-), 193.9(-) ppm. HRMS (ESI): found 306.0126; calc for $\mathrm{C}_{14} \mathrm{H}_{12} \mathrm{NO}_{2} \mathrm{Br}[\mathrm{M}+\mathrm{H}]^{+} 306.0124$.<smiles>COc1ccc(Cc2ccc(N(C)C)cc2)c(C(C)(C)O)c1</smiles>

\section{2-(2-(4-(Dimethylamino)benzyl)-5-methoxyphenyl)-propan-2-ol (402)}

Into a Schlenk flask charged with a solution of compound 403 (516 mg, $1.6 \mathrm{mmol}$ ) in THF (10 mL), 2.2 M BuLi in hexanes $(2.5 \mathrm{~mL}, 5.4 \mathrm{mmol})$ was added dropwise at $-78{ }^{\circ} \mathrm{C}$. After stirring for $40 \mathrm{~min}$ at $-78{ }^{\circ} \mathrm{C}$, acetone $(1.30 \mathrm{~g}, 22.4 \mathrm{mmol})$ was slowly added, and the reaction mixture was allowed to warm up to r.t. An excess of sat. aq. $\mathrm{NH}_{4} \mathrm{Cl}$ was added, and the mixture was extracted with EtOAc $(3 \times 50 \mathrm{~mL})$. The combined organic solutions were dried with $\mathrm{Na}_{2} \mathrm{SO}_{4}$ and evaporated in vacuo. The title compound was isolated as a colorless oil (453 mg, 95\%) by column chromatography (100 g $\mathrm{SiO}_{2}$, hexane/EtOAc, 2:1). ${ }^{1} \mathrm{H} \mathrm{NMR}\left(300 \mathrm{MHz}, \mathrm{CDCl}_{3}\right.$ ): $\delta=1.63$ (s, $6 \mathrm{H}, \mathrm{CMe}_{2}$ ), 2.91 (s, $6 \mathrm{H}$, $\mathrm{NMe}_{2}$ ), 3.81 (s, $3 \mathrm{H}, \mathrm{OMe}$ ), 4.24 (s, $2 \mathrm{H}, \mathrm{CH}_{2}$ ), 6.69 (m, $2 \mathrm{H}, \mathrm{Ar}$ ), 6.73 (dd, $J=8.5$ and $2.7 \mathrm{~Hz}, 1 \mathrm{H}, \mathrm{Ar}), 6.97$ (m, $2 \mathrm{H}, \mathrm{Ar}), 7.05$ (m, $2 \mathrm{H}, \mathrm{Ar}) \mathrm{ppm} .{ }^{13} \mathrm{C}$ NMR (125.7 MHz, $\left.\mathrm{CDCl}_{3}\right): \delta=31.6(+), 37.9(-), 40.8(+), 55.2(+), 73.8(-), 111.3(+), 112.3(+), 113.0(+)$, 129.3(+), 130.9(-), 134.1(+), 147.5(-), 149.0(-), 157.6(-) ppm. HRMS (ESI): found 322.1778; calc for $\mathrm{C}_{19} \mathrm{H}_{25} \mathrm{NO}_{2}[\mathrm{M}+\mathrm{Na}]^{+} 322.1778$.<smiles>COc1ccc(Cc2ccc(N(C)C)cc2)c(Br)c1</smiles>

\section{4-(2-Bromo-4-methoxybenzyl)- $N, N$-dimethylaniline (403)}

Compound $\mathbf{4 0 3}$ was prepared according to the published method. ${ }^{[164]}$ To a cooled $\left(0{ }^{\circ} \mathrm{C}\right)$ solution of compound 4 (1.6 g, $4.8 \mathrm{mmol})$ in THF (45 mL), $\mathrm{AlCl}_{3}(1.7 \mathrm{~g}, 13 \mathrm{mmol})$ was added in one portion, followed by $\mathrm{NaBH}_{4}$ (890 mg, $23.5 \mathrm{mmol}$ ). The mixture was heated with reflux for $2 \mathrm{~h}$, and then water $(25 \mathrm{~mL})$ was carefully added dropwise at $0{ }^{\circ} \mathrm{C}$. The reaction mixture was extracted with $\mathrm{CH}_{2} \mathrm{Cl}_{2}(4 \times 50 \mathrm{~mL})$ and combined organic solutions were dried with $\mathrm{Na}_{2} \mathrm{SO}_{4}$ and evaporated in vacuo. Purification on $\mathrm{SiO}_{2}$ (150 g; 
hexane/EtOAc, 6:1) furnished $970 \mathrm{mg}$ (65\%) of the titled compound. ${ }^{1} \mathrm{H}$ NMR (300 MHz, $\mathrm{CDCl}_{3}$ ): $\delta=2.93$ (s, $6 \mathrm{H}, \mathrm{NMe}_{2}$ ), 3.78 (s, $3 \mathrm{H}, \mathrm{OMe}$ ), 3.96 (s, $2 \mathrm{H}, \mathrm{CH}_{2}$ ), 6.71 (m, $2 \mathrm{H}$, Ar), 6.78 (dd, $J=8.5$ and 2.6 Hz, $1 \mathrm{H}, \mathrm{Ar}$ ), 7.05 (m, $3 \mathrm{H}, \mathrm{Ar}$ ), 7.12 (d, $J=2.6 \mathrm{~Hz}, 1 \mathrm{H}$, Ar) ppm. ${ }^{13} \mathrm{C}$ NMR $\left(100.7 \mathrm{MHz}, \mathrm{CDCl}_{3}\right): \delta=39.8(-), 40.8(+), 55.5(+), 110.0(-)$, 113.0(+), 113.6(+), 117.8(+), 124.8(-), 129.5(+), 131.2(+), 133.3(-), 149.1(-), 158.3(-) ppm. HRMS (ESI): found 320.0648; calc for $\mathrm{C}_{16} \mathrm{H}_{18} \mathrm{NOBr}[\mathrm{M}+\mathrm{H}]^{+} 320.0645$.<smiles>COc1ccc(C(=O)c2ccc(N(C)C)cc2)c(Br)c1</smiles>

\section{(2-Bromo-4-methoxyphenyl)(4-(dimethylamino)phenyl)-methanone (404)}

Compound 404 was prepared according to the known procedure. ${ }^{[159]}$ To a mixture of compound 401 (2.0 g, $6.5 \mathrm{mmol}$ ) and paraformaldehyde (1.96 g, $65 \mathrm{mmol}$ ) in AcOH (50 $\mathrm{mL}), \mathrm{NaBH}_{3} \mathrm{CN}\left(1.97 \mathrm{~g}, 31.3 \mathrm{mmol}\right.$ ) was added in one portion at $0{ }^{\circ} \mathrm{C}$. The reaction mixture was stirred overnight at r.t., and then an additional amount of $\mathrm{NaBH}_{3} \mathrm{CN}$ (1.00 g, $15.9 \mathrm{mmol}$ ) was added. After stirring for $2 \mathrm{~h}$ at r.t., the reaction mixture was transferred into an excess of $15 \% \mathrm{NaOH}$ with ice, $\mathrm{pH}$ was adjusted to 12 with solid $\mathrm{NaOH}$, and the solution was extracted with $\mathrm{CH}_{2} \mathrm{Cl}_{2}(3 \times 100 \mathrm{~mL})$. The combined organic solutions were dried with $\mathrm{Na}_{2} \mathrm{SO}_{4}$ and evaporated in vacuo to give $2.06 \mathrm{~g}$ (83\%) of the title compound as a yellow oil. ${ }^{1} \mathrm{H}$ NMR (300 MHz, $\mathrm{CDCl}_{3}$ ): $\delta=3.08$ (s, $6 \mathrm{H}, \mathrm{NMe}_{2}$ ), 3.15 (s, $3 \mathrm{H}, \mathrm{OMe}$ ), 6.64 (m, 2 H, Ar), 6.90 (dd, $J=8.5$ and $2.4 \mathrm{~Hz}, 1 \mathrm{H}, \mathrm{Ar}$ ), 7.16 (d, $J=2.4 \mathrm{~Hz}, 1 \mathrm{H}, \mathrm{Ar}$ ), 7.26 (d, $J=8.5 \mathrm{~Hz}, 1 \mathrm{H}, \mathrm{Ar}), 7.71$ (m, $2 \mathrm{H}, \mathrm{Ar}) .{ }^{13} \mathrm{C}$ NMR (100.7 MHz, $\left.\mathrm{CDCl}_{3}\right): \delta=$ 40.1(+), 55.6(+), 110.7(+), 113.0(+), 118.2(+), 120.6(-), 124.4(-), 130.1(+), 132.6(+), 134.0(-), 153.6(-), 160.6(-), 193.7(-). HRMS (ESI): found 320.0432; calc for $\mathrm{C}_{16} \mathrm{H}_{16} \mathrm{NO}_{2} \mathrm{Br}[\mathrm{M}+\mathrm{H}]^{+} 334.0437$.<smiles>COc1ccc2c(c1)C(C)(C)c1cc(N(C)C)ccc1C2=O</smiles>

\section{3-(Dimethylamino)-6-methoxy-10,10-dimethylanthracen-9(10H)-one (405)}

Cyclization of compound $\mathbf{4 0 2}$ to 1,2-dihydroanthracene derivative was performed according to the known method, ${ }^{[165]}$ and further oxidation to anthracenone 405 was carried out as described. ${ }^{[156 \mathrm{~d}]}$ To a solution of compound $\mathbf{4 0 2}$ (100 mg, $\left.0.33 \mathrm{mmol}\right)$ in dry $\mathrm{CH}_{2} \mathrm{Cl}_{2}(4 \mathrm{~mL}), \mathrm{AlCl}_{3}(110 \mathrm{mg}, 0.825 \mathrm{mmol})$ was added at $0{ }^{\circ} \mathrm{C}$. The mixture was stirred for $6 \mathrm{~h}$ at $0{ }^{\circ} \mathrm{C}$ and for $10 \mathrm{~h}$ at r.t. After quenching with $1 \mathrm{M}$ aq. $\mathrm{NaOH}(4 \mathrm{~mL})$, the 
organic layer was separated and the aqueous solution was extracted with $\mathrm{CH}_{2} \mathrm{Cl}_{2}(3 \times 10$ $\mathrm{mL}$ ). Combined organic solutions were dried with $\mathrm{Na}_{2} \mathrm{SO}_{4}$ and evaporated in vacuo. The residue was dissolved in acetone $(4 \mathrm{~mL})$, the solution was cooled down to $-18^{\circ} \mathrm{C}$, and a powder of $\mathrm{KMnO}_{4}(110 \mathrm{mg}, 0.70 \mathrm{mmol})$ was added in small portions over $2 \mathrm{~h}$. The reaction mixture was diluted with 2 volumes of $\mathrm{CH}_{2} \mathrm{Cl}_{2} ; \mathrm{MnO}_{2}$ was filtered off and washed with $\mathrm{CH}_{2} \mathrm{Cl}_{2}$, and the filtrate was evaporated to dryness. The title compound was isolated by column chromatography (50 $\mathrm{g} \mathrm{SiO}_{2}$; hexane/EtOAc, 4:1) as a yellow solid (30 $\mathrm{mg}$, 31\%). ${ }^{1} \mathrm{H}$ NMR (300 MHz, $\mathrm{CDCl}_{3}$ ): $\delta=1.71$ (s, $6 \mathrm{H}, \mathrm{CMe}_{2}$ ), 3.12 (s, $6 \mathrm{H}, \mathrm{NMe}_{2}$ ), 3.91 (s, $3 \mathrm{H}, \mathrm{OMe}$ ), 6.77 (m, $2 \mathrm{H}, \mathrm{Ar}$ ), 6.95 (dd, $J=2.5$ and $8.7 \mathrm{~Hz}, 1 \mathrm{H}, \mathrm{Ar}$ ), 7.09 (d, $J=2.5 \mathrm{~Hz}$, $1 \mathrm{H}, \mathrm{Ar}$ ), 8.26 (d, $J=8.40 \mathrm{~Hz}, 1 \mathrm{H}, \mathrm{Ar}), 8.34$ (d, $J=8.7 \mathrm{~Hz}, 1 \mathrm{H}$, Ar) ppm. ${ }^{13} \mathrm{C} \mathrm{NMR}$ $\left(125.7 \mathrm{MHz}, \mathrm{CDCl}_{3}\right): \delta=33.5(+), 38.2(-), 40.2(+), 55.4(+), 107.5(+), 110.9(+), 111.5(+)$, 112.2(+), 119.4(-), 124.3(-), 129.4(+), 129.5(+), 152.2(-), 152.5(-), 153.2(-), 162.9(-), 181.2(-) ppm. HRMS (ESI): found 296.1649; calc for $\mathrm{C}_{19} \mathrm{H}_{21} \mathrm{NO}_{2}[\mathrm{M}+\mathrm{H}]^{+}$296.1645.<smiles>CN=C1C=CC(=C2C3=C(C(=O)c4ccccc43)C(C)(C)c3cc(OC)ccc32)C=C1</smiles>

N-(10-(2-((2-Amino-2-methylpropoxy)carbonyl)phenyl)-7-methoxy-9,9-dimethylanthracen-2(9H)-ylidene)- $N$-methyl methanaminium chloride (407)

Into a Schlenk flask charged with a solution of 2-(2-bromophenyl)-4,4-dimethyl-4,5-dihydrooxazole (926 mg, $3.60 \mathrm{mmol})$ in THF (15 mL), 1.5 M tBuLi in pentane (2.6 mL, 3.9 mmol) was added dropwise at $-78{ }^{\circ} \mathrm{C}$ to form the organolithium compound 406. The mixture was stirred for $40 \mathrm{~min}$ at $-78{ }^{\circ} \mathrm{C}$, and a solution of ketone 405 (215 $\mathrm{mg}, 0.73$ mmol) in THF $(8 \mathrm{~mL})$ was added dropwise. The reaction mixture was stirred at $-78{ }^{\circ} \mathrm{C}$ for $1 \mathrm{~h}$ at r.t. and finally transferred into a cooled $\left(0^{\circ} \mathrm{C}\right)$ mixture of $\mathrm{MeOH}$ and $\mathrm{AcOH}(15$ and $2 \mathrm{~mL}$, respectively). The residue after complete evaporation of the reaction mixture was subjected to column chromatography (100 g SiO, $\mathrm{MeCN} / \mathrm{H}_{2} \mathrm{O}$ (both with $0.1 \% \mathrm{v} / \mathrm{v}$ TFA), 10:1 $\rightarrow$ 10:1). Fractions containing the title compound were combined, evaporated in vacuo, and residue was dissolved in $\mathrm{CH}_{2} \mathrm{Cl}_{2}$ and washed with brine and sat. aq. $\mathrm{NH}_{4} \mathrm{Cl}$. Evaporation of $\mathrm{CH}_{2} \mathrm{Cl}_{2}$ gave $270 \mathrm{mg}$ (73\%) of the title compound. ${ }^{1} \mathrm{H}$ NMR (300 MHz, $\mathrm{CDCl}_{3}$ ): $\delta=1.36$ (s, $3 \mathrm{H}, \mathrm{Me}$ ), 1.43 (s, $3 \mathrm{H}, \mathrm{Me}$ ), 1.75 (s, $3 \mathrm{H}, \mathrm{Me}$ ), 1.84 (s, $3 \mathrm{H}, \mathrm{Me}$ ), 3.43 (s, 3 H, NMe), 3.53 (s, 3 H, NMe), 3.94 (s, 3 H, OMe), 4.05 (d, J = 11.7 Hz, 1 H), 
4.22 (d, $J=11.7$ Hz, 1 H), 5.82 (br. s, $\mathrm{NH}_{2}$ ), 6.83 (m, 2 H, Ar), 7.17 (m, 2 H, Ar), 7.29 (d, $J=2.4 \mathrm{~Hz}, 1 \mathrm{H}, \mathrm{Ar}), 7.72$ (m, $2 \mathrm{H}, \mathrm{Ar}), 8.69$ (m, $1 \mathrm{H}, \mathrm{Ar}), 8.86$ (m, $2 \mathrm{H}, \mathrm{Ar}) \mathrm{ppm} .{ }^{13} \mathrm{C}$ NMR (75.5 MHz, $\left.\mathrm{CDCl}_{3}\right): \delta=22.7(-), 22.8(+), 31.6(+), 31.9(-), 35.0\left(^{+}\right), 41.9\left(^{+}\right)$, 42.0(-), 53.3(-), 56.1(+), 69.7(-), 112.2(+), 113.6(+), 115.7(+), 122.7(-), 124.3(-), 129.5(-), 129.6(+), 130.4(+), 132.5(+), 132.6(+), 135.9(+), 136.8(-), 139.9(-), 154.3(-), 158.8(-), 160.1(-), 164.4(-), 166.0(-), 168.1(-) ppm. HRMS (ESI): found 471.2633; calc. for $\mathrm{C}_{30} \mathrm{H}_{35} \mathrm{~N}_{2} \mathrm{O}_{3}\left[\mathrm{M}^{+}\right] 471.2642$.<smiles>[R]Oc1ccc2c(c1)C(C)(C)c1cc(N(C)C)ccc1C21OC(=O)c2ccccc21</smiles>

3- $N, N$-dimethylamino-6-methoxy-10,10-dimethyl-3' $H, 10 H$-spiro[anthracene-9,1'isobenzofuran]-3'-one (408-Me) and $3-N, N$-dimethylamino-6-hydroxy-10,10-dimethyl-3' $H, 10 H$-spiro[anthracene-9,1'-isobenzofuran]-3'-one (408-H)

A solution of compound 407 (200 mg, $0.40 \mathrm{mmol}$ ) in 20\% aq $\mathrm{HCl}(30 \mathrm{~mL}$ ) was stirred at $80{ }^{\circ} \mathrm{C}$ for $6.5 \mathrm{~h}$. After cooling to r.t., the reaction mixture was neutralized with solid $\mathrm{NaHCO}_{3}$ and extracted with $\mathrm{CH}_{2} \mathrm{Cl}_{2}(4 \times 20 \mathrm{~mL})$. Combined organic extracts were dried with $\mathrm{Na}_{2} \mathrm{SO}_{4}$ and evaporated. The residue was subjected to column chromatography (100 $\mathrm{g} \mathrm{SiO}_{2}$, hexane/EtOAc, 2:1) to furnish $68 \mathrm{mg}$ (43\%) of 408-Me and $40 \mathrm{mg}$ (26\%) of 408$\mathrm{H}$ as colorless powders.

408-Me: ${ }^{1} \mathrm{H}$ NMR (300 MHz, $\mathrm{CDCl}_{3}$ ): $\delta=1.76$ (s, $3 \mathrm{H}, \mathrm{Me}$ ), 1.87 (s, $3 \mathrm{H}, \mathrm{Me}$ ), 2.99 (s, 6 H, $\mathrm{NMe}_{2}$ ), 3.82 (s, 3 H, OMe), 6.50-6.65 (m, 2 H, Ar), 6.68 (m, 2 H, Ar), 6.92 (m, 1 H, Ar), 7.05 (m, 1 H, Ar), 7.15 (m, 1 H, Ar), 7.58 (m, 2 H, Ar), 8.00 (m, 1 H, Ar) ppm. ${ }^{13} \mathrm{C}$ NMR (75.5 MHz, $\left.\mathrm{CDCl}_{3}\right): \delta=32.4(+), 35.5(+), 38.6(-), 40.5(+), 55.3(+), 111.8(+)$, 112.3(+), 123.8(+), 124.4(-), 124.9(+), 126.9(-), 128.9(+), 129.3(+), 134.5(+), 146.4(-), 147.5(-), 155.3(-), 159.9(-), 170.6(-) ppm. HRMS (ESI): found 400.1898; calc. for $\mathrm{C}_{26} \mathrm{H}_{25} \mathrm{NO}_{3}[\mathrm{M}+\mathrm{H}]^{+}$400.1907. Spectal data in $\mathrm{MeOH}$ with $0.1 \% \mathrm{v} / \mathrm{v}$ TFA: $\lambda_{\text {abs, }} \mathrm{nm}(\varepsilon$, $\left.\mathrm{M}^{-1} \mathrm{~cm}^{-1}\right)=482 \mathrm{sh}$ (10290), 513 (18250), 549 (18430), $\lambda_{\mathrm{em}}=585 \mathrm{~nm}, \Phi_{\mathrm{fl}}=0.01$ (standard: $\mathrm{RDC}, \Phi_{\mathrm{fl}}=0.33$ in THF).

408-H: ${ }^{1} \mathrm{H}$ NMR (600 MHz, $\mathrm{CDCl}_{3}$ ): $\delta=1.56$ (s, $\left.3 \mathrm{H}, \mathrm{Me}\right), 1.73$ (s, $3 \mathrm{H}, \mathrm{Me}$ ), 3.17 (s, 6 H, $\mathrm{NMe}_{2}$ ), 6.64 (m, 2 H, Ar), 6.88 (m, 1 H, Ar), 7.03 (m, 2 H, Ar), 7.12 (m, 1 H, Ar), 7.61 (m, 2 H, Ar), 7.68 (m, 1 H, Ar), 8.05 (m, 1 H, Ar). HRMS (ESI): found 386.1747, calc. for $\mathrm{C}_{25} \mathrm{H}_{23} \mathrm{NO}_{3}[\mathrm{M}+\mathrm{H}]^{+}$386.1751. Spectral data in $\mathrm{MeOH}: \lambda_{\text {abs }}\left(\varepsilon, \mathrm{M}^{-1} \mathrm{~cm}^{-1}\right)=561(620)$, 
$\lambda_{\text {em }}=598 \mathrm{~nm}, \Phi_{\mathrm{fl}}=0.85$ (standard: rhodamine $630, \Phi_{\mathrm{fl}}=0.97$ in ethanol). Low absorption in the visible range is explained by the fact that under neutral conditions, the equilibrium between the colorless "closed" form and the colored "open" form is shifted toward the former one (Scheme 28a). Spectral data in $\mathrm{MeOH}$ with $0.1 \% \mathrm{v} / \mathrm{v}$ TFA: $\lambda_{\text {abs }}, \mathrm{nm}\left(\varepsilon, \mathrm{M}^{-}\right.$ $\left.{ }^{1} \mathrm{~cm}^{-1}\right)=483 \mathrm{sh}(13500), 515(30000), 553$ (37400), $\lambda_{\text {em }}=585 \mathrm{~nm}\left(\right.$ broad), $\Phi_{\mathrm{fl}}=0.11$ (standard: perylene diimide $\mathrm{KP} 174, \Phi_{\mathrm{fl}}=0.58$ in water). Spectral data in $\mathrm{MeOH}$ with $0.1 \% \mathrm{v} / \mathrm{v} \mathrm{Et}_{3} \mathrm{~N}: \lambda_{\mathrm{abs}}, \mathrm{nm}\left(\varepsilon, \mathrm{M}^{-1} \mathrm{~cm}^{-1}\right)=560$ (34300), $\lambda_{\mathrm{em}}=598 \mathrm{~nm}, \Phi_{\mathrm{fl}}=0.94$ (standard: perylene diimide $\mathrm{KP} 174, \Phi_{\mathrm{fl}}=0.58$ in water).<smiles>[R]OC(=O)CCCN(C)C(=O)c1ccccc1C1=C2C=CC(=O)C=C2C(C)(C)c2cc(N(C)C)ccc21</smiles>

Methyl 4-(2-(6- $N, N$-Dimethylamino-10,10-dimethyl-3-oxo-3,10-dihydroanthracen-9yl)- $N$ '-methylbenzamido)butanoate (409-Me) and 4-(2-(6- $N, N$-dimethylamino)10,10-dimethyl-3-oxo-3,10-dihydroanthracen-9-yl)- $N$ '-methylbenzamido)butanoic acid (409-H)

Into a Schlenk flask charged with a solution of compound 410 (36 mg, $0.065 \mathrm{mmol}$ ) in $\mathrm{CH}_{2} \mathrm{Cl}_{2}$ (5 mL), $1 \mathrm{M}$ solution of $\mathrm{BBr}_{3}$ in $\mathrm{CH}_{2} \mathrm{Cl}_{2}(400 \mu \mathrm{L}, 0.4 \mathrm{mmol})$ was added dropwise at r.t. The reaction mixture was stirred for $1 \mathrm{~h}$ and quenched with sat. aq. $\mathrm{NaHCO}_{3}$ (15 mL). The organic layer was separated, and the aqueous phase was saturated with $\mathrm{NH}_{4} \mathrm{Cl}$ and extracted with $\mathrm{CH}_{2} \mathrm{Cl}_{2}(5 \times 30 \mathrm{~mL})$. Combined organic solutions were dried with $\mathrm{Na}_{2} \mathrm{SO}_{4}$ and evaporated. Column chromatography (40 g SiO $2, \mathrm{CH}_{2} \mathrm{Cl}_{2} / \mathrm{MeOH}, 20: 1$, then $\mathrm{MeCN} / \mathrm{H}_{2} \mathrm{O}$, 5:1) afforded $16 \mathrm{mg}(49 \%)$ of $409-\mathrm{Me}$ and $11 \mathrm{mg}(23 \%)$ of $409-\mathrm{H}$ as dark-violet solids.

409-Me was an 1:2.5 mixture of 2 diastereomers. ${ }^{1} \mathrm{H}$ NMR (300 MHz, $\mathrm{CD}_{3} \mathrm{CN}$, only the signals of the major isomer are given): $\delta=1.46$ (m, $2 \mathrm{H}, \mathrm{CH}_{2}$ ), 1.55 (s, $3 \mathrm{H}, \mathrm{Me}$ ), 1.68 (s, $3 \mathrm{H}, \mathrm{Me}$ ), 1.86-1.94 (m, $2 \mathrm{H}, \mathrm{CH}_{2}$ ), 2.78 (s, $3 \mathrm{H}, \mathrm{NMe}$ ), 3.09 (s, $6 \mathrm{H}, \mathrm{NMe}_{2}$ ), 3.10-3.32 (m, 2 H, NCH ), 3.53 (s, 3 H, COOMe), 6.17 (dd, $J=9.6$ and $2.0 \mathrm{~Hz}, 1 \mathrm{H}, \mathrm{Ar}$ ), 6.55 (dd, $J=9.1$ and $2.6 \mathrm{~Hz}, 1 \mathrm{H}, \mathrm{Ar}), 6.66(\mathrm{~m}, J=2.0 \mathrm{~Hz}, 1 \mathrm{H}, \mathrm{Ar}), 6.81$ (m, $J=9.1 \mathrm{~Hz}, 1 \mathrm{H}, \mathrm{Ar}$ ), 6.92 (m, $J=9.6 \mathrm{~Hz}, 1 \mathrm{H}, \mathrm{Ar}), 6.97$ (m, $J=2.6 \mathrm{~Hz}, 1 \mathrm{H}, \mathrm{Ar}$ ), 7.31 (m, $1 \mathrm{H}, \mathrm{Ar}$ ), 7.45 (m, 1 $\mathrm{H}, \mathrm{Ar}$ ), 7.56 (m, $2 \mathrm{H}, \mathrm{Ar}$ ) ppm. ${ }^{13} \mathrm{C} \mathrm{NMR}$ (75.5 MHz, $\left.\mathrm{CD}_{3} \mathrm{CN}\right): \delta=22.6(-), 31.4(-)$, 31.8(+), 35.5(+), 37.6(+), 40.5(+), 40.9(-), 46.5(-), 51.8(+), 110.4(+), 111.3(+), 120.6(-), 
120.9(-), 121.9(-), 123.5(+), 124.9(+), 127.6(+), 129.1(+), 129.5(+), 131.4(+), 134.8(+), 136.1(-), 137.7(-), 139.0(+), 152.1(-), 154.2(-), 157.2(-), 169.4(-), 174.1(-), 184.2(-) ppm. HRMS (ESI): found 499.2592; calc. for $\mathrm{C}_{31} \mathrm{H}_{35} \mathrm{~N}_{2} \mathrm{O}_{4}\left[\mathrm{M}^{+}\right]$499.2591. Lifetime of the excited state $(\tau): 2.2$ ns (aq. PBS buffer).

409-H was an 1:2.5 mixture of two diastereomers, ${ }^{1} \mathrm{H}$ NMR (300 MHz, $\mathrm{CD}_{3} \mathrm{CN}$, only signals of the major isomer are given): $\delta=1.38$ (m, $\left.1 \mathrm{H}, \mathrm{CH}_{2}\right), 1.43-1.57\left(\mathrm{~m}, 1 \mathrm{H}, \mathrm{CH}_{2}\right.$ ), 1.53 (s, 3 H, Me), 1.66 (s, 3 H, Me), 1.83 (m, 1 H, CH${ }_{2} \mathrm{COOMe}$ ), 1.93-2.00 (m, 1 H, $\mathrm{CH}_{2} \mathrm{COOMe}$ ), 3.09 (s, $3 \mathrm{H}, \mathrm{NMe}$ ), 3.09-3.23 (m, $2 \mathrm{H}, \mathrm{NCH}_{2}$ ), 3.27 (s, $6 \mathrm{H}, \mathrm{NMe}_{2}$ ), 6.23 (dd, $J=9.6$ and $2.1 \mathrm{~Hz}, 1 \mathrm{H}, \mathrm{Ar}$ ), 6.55 (dd, $J=9.1$ and $2.6 \mathrm{~Hz}, 1 \mathrm{H}, \mathrm{Ar}$ ), 6.72 (m, $1 \mathrm{H}$, Ar), 6.81 (m, $J=9.1 \mathrm{~Hz}, 1 \mathrm{H}, \mathrm{Ar}$ ), 6.95-6.99 (m, 2 H, Ar), 7.30 (m, 1 H, Ar), 7.45 (m, 1 $\mathrm{H}, \mathrm{Ar}), 7.56$ (m, $2 \mathrm{H}, \mathrm{Ar}) .{ }^{13} \mathrm{C} \mathrm{NMR}\left(75.5 \mathrm{MHz}, \mathrm{CD}_{3} \mathrm{CN}\right): \delta=23.0(-), 32.0(+), 32.7(-)$, 35.8(+), 37.9(+), 40.5(+), 47.1(-), 110.4(+), 111.5(+), 120.4(-), 121.5(-), 123.1(+), 124.5(+), 127.8(+), 129.5(+), 129.7(+), 131.4(+), 135.5(+), 135.6(-), 137.3(-), 140.0(+), 153.3(-), 154.6(-), 158.6(-), 170.1(-), 185.7(-) ppm. HRMS (ESI): found 485.2433; calc for $\mathrm{C}_{30} \mathrm{H}_{32} \mathrm{~N}_{2} \mathrm{O}_{4}[\mathrm{M}+\mathrm{H}]^{+} 485.2435$.

\section{Preparation of 409-H from 409-Me}

Compound 409-Me (4 mg; $8 \mu \mathrm{mol})$ was stirred for $1 \mathrm{~h}$ at r.t. in a 10:15:0.7 mixture of $\mathrm{H}_{2} \mathrm{O}, \mathrm{THF}$, and $1 \mathrm{M}$ aq. $\mathrm{NaOH}(2.5 \mathrm{~mL})$. Then $\mathrm{AcOH}(1 \mathrm{~mL})$ was added, and the reaction mixture was evaporated to dryness. Purification by flash chromatography (15 g $\mathrm{SiO}_{2}$, $\mathrm{MeCN} / \mathrm{H}_{2} \mathrm{O}$, 5:1) followed by evaporation, dissolution in $\mathrm{CH}_{2} \mathrm{Cl}_{2}$, and filtration through a fine glass filter afforded $4 \mathrm{mg}$ (quantitative yield) of the solid title compound. HPLC: B/A $=30 / 70$ to 100/0 in $25 \mathrm{~min}$, detection at $550 \mathrm{~nm}, t_{\mathrm{R}}=7.6 \mathrm{~min}$ (100\%). Data in methanol: $\lambda_{\text {abs }}\left(\varepsilon, \mathrm{M}^{-1} \mathrm{~cm}^{-1}\right)=573 \mathrm{~nm}(41000), \lambda_{\mathrm{em}}=613 \mathrm{~nm}, \Phi_{\mathrm{fl}}=0.64, \tau=3.95 \mathrm{~ns}$. Excitation spectrum in methanol: emission at $625 \mathrm{~nm}$ was monitored and found to be maximal at $\lambda_{\text {excit }}=588 \mathrm{~nm}$. Data in PBS buffer at pH 7.4: $\lambda_{\text {abs }}=586 \mathrm{~nm} ; \varepsilon=58600 \mathrm{M}^{-1} \mathrm{~cm}^{-1}, \lambda_{\text {em }}=$ $613 \mathrm{~nm}, \Phi_{\mathrm{fl}}=0.32$ (standard: perylene diimide, $\Phi_{\mathrm{fl}}=0.58$ in $\mathrm{H}_{2} \mathrm{O}$ ), $\tau=2.54$ ns.<smiles>CN(CCCC(=O)ON1C(=O)CCC1=O)C(=O)c1ccccc1C1=C2C=CC(=O)C=C2C(C)(C)c2cc(N(C)C)ccc21</smiles>

2,5-Dioxopyrrolidin-1-yl 4-(2-(6- $N, N$-dimethylamino)-10,10-dimethyl-3-oxo-3,10-dihydro-anthracen-9-yl)- $N$ '-methyl benzamido)butanoate (409-NHS) 
To a stirred solution of compound 409-H (3 mg, $6.2 \mu \mathrm{mol}$ ) and $N$-hydroxysuccinimide (11 mg, $93 \mu \mathrm{mol})$ in MeCN/DMF mixture (2:1, $2 \mathrm{~mL})$, HATU (10 mg, $25 \mu \mathrm{mol})$ was added followed by $\mathrm{NEt}_{3}(16 \mu \mathrm{L}, 112 \mu \mathrm{mol})$. The reaction mixture was stirred overnight and evaporated in vacuo. The residue was subjected to flash chromatography on $\mathrm{SiO}_{2}(20$ g, $\left.\mathrm{MeCN} / \mathrm{H}_{2} \mathrm{O}, 10: 1\right)$. The main fraction was filtered through a fine glass filter and freezedried to give $3.1 \mathrm{mg}$ (86\%) of the title compound. HPLC: B/A = 30/70 to 100/0 in 25 min, detection at $550 \mathrm{~nm}, t_{\mathrm{R}}=9.7 \mathrm{~min}$ (100\%). HRMS (ESI): found 582.2605; calc for $\mathrm{C}_{34} \mathrm{H}_{35} \mathrm{~N}_{3} \mathrm{O}_{6}[\mathrm{M}+\mathrm{H}]^{+}$582.2599. The conjugate with goat antirabbit antibodies (AB256): $\lambda_{\mathrm{abs}}=585 \mathrm{~nm}, \lambda_{\mathrm{em}}=613 \mathrm{~nm}, \mathrm{DOL}=10.9, \Phi_{\mathrm{fl}}=0.04$ in PBS buffer at pH 7.4 (standard: rhodamine 101, $\left.\Phi_{\mathrm{fl}}=1 \mathrm{in} \mathrm{EtOH}\right)$. The conjugate with goat antirabbit antibodies (AB257): $\lambda_{\mathrm{abs}}=586 \mathrm{~nm}, \lambda_{\mathrm{em}}=614 \mathrm{~nm}, \mathrm{DOL}=12.4, \Phi_{\mathrm{fl}}=0.04$ in PBS buffer at pH 7.4 (standard: perylene diimide $\mathrm{KP} 174, \Phi_{\mathrm{fl}}=0.58$ in $\mathrm{H}_{2} \mathrm{O}$, excitation at $550 \mathrm{~nm}$ ).<smiles>COC(=O)CCCN(C)C(=O)c1ccccc1C1=C2C=CC(=[N+]=[N-])C=C2C(C)(C)c2cc(OC)ccc21</smiles>

$N$-(7-Methoxy-10-(2-(N'-(4-methoxy-4-oxobutyl)-N'-methylcarbamoyl)phenyl)-9,9dimethylanthracen-2(9H)-ylidene)- $N$-methylmethanaminium (410)

Into a Schlenk flask charged with a solution of compound 408-Me (34 mg, $0.085 \mathrm{mmol}$ ) in dichloroethane $(3 \mathrm{~mL}), \mathrm{POCl}_{3}(390 \mu \mathrm{L}, 4.3 \mathrm{mmol})$ was injected. After stirring at $80{ }^{\circ} \mathrm{C}$ for $2 \mathrm{~h}$, all volatile materials were evaporated in vacuo and the solid residue was dissolved in $\mathrm{CH}_{3} \mathrm{CN}$ (5 mL). A solution of methyl 4- $\mathrm{N}$-methylaminobutyrate hydrochloride (413) (71 mg, $0.425 \mathrm{mmol}$ ) in MeCN (3 mL) was added into the flask, followed by $\mathrm{NEt}_{3}$ $(360 \mu \mathrm{L}, 2.5 \mathrm{mmol})$. After stirring at r.t. for $15 \mathrm{~min}$, all volatile materials were removed in vacuo, and the residue was dissolved in $\mathrm{CH}_{2} \mathrm{Cl}_{2}(20 \mathrm{~mL})$ and washed with a satd aq solution of $\mathrm{NH}_{4} \mathrm{Cl}$. The organic layer was separated, dried with $\mathrm{Na}_{2} \mathrm{SO}_{4}$, and evaporated. The titled compound (36 mg, 76\%) was isolated by column chromatography (40 g $\mathrm{SiO}_{2}$, $\left.\mathrm{MeCN} / \mathrm{H}_{2} \mathrm{O}, 15: 1\right)$ as a red solid. ${ }^{1} \mathrm{H}$ NMR (300 MHz, $\left.\mathrm{CD}_{3} \mathrm{CN}\right): \delta=1.34$ (m, $2 \mathrm{H}, \mathrm{CH}_{2}$ ), 1.65 (s, 3 H, Me), 1.72-1.82 (m, 2 H, CH ), 1.80 (s, 3 H, Me), 2.86 (s, 3 H, NMe), 3.113.23 (m, 2 H, NCH ), 3.44 (s, 6 H, NMe $)$ ), 3.53 (s, 3 H, COOMe), 3.94 (s, 3 H, OMe), 6.93 (m, 2 H, Ar), 7.17-7.24 (m, 2 H, Ar), 7.27 (m, 1 H, Ar), 7.39 (m, 1 H, Ar), 7.41 (m, $1 \mathrm{H}, \mathrm{Ar}), 7.56$ (m, $1 \mathrm{H}, \mathrm{Ar}), 7.66$ (m, $2 \mathrm{H}, \mathrm{Ar}) \mathrm{ppm} .{ }^{13} \mathrm{C} \mathrm{NMR}\left(75.5 \mathrm{MHz}, \mathrm{CD}_{3} \mathrm{CN}\right): \delta=$ 
22.5(-), $31.4(-), 32.0(+), 35.0(+), 37.6(+), 42.6(+), 42.8(-), 46.7(-), 51.9(+), 57.0(+)$, 114.4(+), 114.5(+), 115.8(-), 116.5(+), 123.5(-), 124.2(-), 125.1(-), 128.0(+), 130.0(+), 130.2(+), 130.5(+), 131.1(+), 135.0(-), 137.0(+), 137.4(-), 141.3(+), 155.2(-), 160.0(-), 160.8(-), 166.8(-), 174.1(-) ppm. HRMS (ESI): found 513.2740; calc. for $\mathrm{C}_{32} \mathrm{H}_{37} \mathrm{~N}_{2} \mathrm{O}_{4}$ $\left[\mathrm{M}^{+}\right]$513.2748.<smiles>COC(=O)CCCN(C)C(=O)c1ccccc1</smiles>

\section{Methyl 4-( $N$-benzyloxycarbonyl- $N$-methylamino)butanoate (412)}

Methylation of $N$-protected butyric acid $\mathbf{4 1 1}$ was performed according to the known procedure. ${ }^{[176]}$ To a solution of compound 411 (4.1 g, $17.4 \mathrm{mmol}$ ) in dry DMF (50 mL) MeI (13 mL, $209 \mathrm{mmol})$ and $\mathrm{Ag}_{2} \mathrm{O}$ (24.2 g, $\left.104 \mathrm{mmol}\right)$ were added under cooling. The resulting mixture was stirred overnight at r.t. Afterwards, the reaction mixture was filtered through a pad of Celite eluting with $\mathrm{CH}_{2} \mathrm{Cl}_{2}(\sim 300 \mathrm{~mL})$. The filtrate was washed with $20 \% \mathrm{Na}_{2} \mathrm{~S}_{2} \mathrm{O}_{3}(2 \times 100 \mathrm{~mL})$ and water $(10 \times 100 \mathrm{~mL})$ dried with $\left.\mathrm{Na} 100 \mathrm{~mL}\right)$ dried with $\mathrm{Na}_{2} \mathrm{SO}_{4}$ and evaporated in vacuo. The crude product (4.5 g, 98\%) was used without further purification. ${ }^{1} \mathrm{H}$ NMR (300 MHz, $\left.\mathrm{CDCl}_{3}\right): \delta=1.77-1.93\left(\mathrm{~m}, 2 \mathrm{H}, \mathrm{CH}_{2}\right), 2.23-2.38$ (m, $2 \mathrm{H}, \mathrm{CH}_{2}$ ), 2.92 (s, $\left.3 \mathrm{H}, \mathrm{NMe}\right), 3.33$ (t, J = 7.0 Hz, $\left.2 \mathrm{H}, \mathrm{NCH}_{2}\right), 3.64$ (m, $3 \mathrm{H}$, COOMe), 5.11 (s, 2 H, CH${ }_{2}$ ), 7.22-7.42 (m, 5 H, Ar) ppm. MS (ESI): $m / z=288$ (100) $[\mathrm{M}+\mathrm{Na}]^{+}$.<smiles>CNCCCC(=O)OC</smiles>

\section{Methyl 4- $N$-methylaminobutanoate hydrochloride (413)}

A dry $100 \mathrm{~mL}$ Schlenk flask was charged with Pd/C (200 mg, $0.19 \mathrm{mmol}, 5 \mathrm{~mol} \%)$ and EtOH (5 mL). The flask was purged with $\mathrm{H}_{2}$, and a solution of compound 412 ( $1 \mathrm{~g}, 3.77$ $\mathrm{mmol})$ in $\mathrm{EtOH}(20 \mathrm{~mL})$ mixed with $5 \mathrm{M} \mathrm{HCl}$ in $\mathrm{PrOH}(1 \mathrm{~mL})$ was added. The resulting suspension was stirred for $3 \mathrm{~h}$ at r.t. under slow flow of hydrogen. Afterwards, the reaction mixture was filtered through a plug of Celite eluting with $\mathrm{CH}_{2} \mathrm{Cl}_{2}(\sim 100 \mathrm{~mL})$. Evaporation of the filtrate gave an oil which was recrystallized from $n$-hexane/ $\mathrm{Et}_{2} \mathrm{O}$ to afford $450 \mathrm{mg}$ (77\%) of a white hygroscopic solid. ${ }^{1} \mathrm{H}$ NMR (300 MHz, $\mathrm{CD}_{3} \mathrm{OD}$ ): $\delta=1.90-2.02$ (m, $2 \mathrm{H}, \mathrm{CH}_{2}$ ), 2.49 (t, $J=7.1 \mathrm{~Hz}, 2 \mathrm{H}, \mathrm{CH}_{2}$ ), 2.70 (s, $3 \mathrm{H}, \mathrm{NMe}$ ), 3.04 (m, $2 \mathrm{H}, \mathrm{CH}_{2}$ ), 3.68 (s, 3 H, COOMe) ppm. 
Fmoc<smiles>CN(C)CCOCCOCC(=O)O</smiles>

2-(2-(2- $N-((9 H-F l u o r e n-9-y l m e t h o x y) c a r b o n y l)-N$-methylaminoethoxy)ethoxy)acetic acid (415-Fmoc)

Methylation of $N$-protected amino acid 414 was carried out according to the known procedure. ${ }^{[166]}$ A mixture of compound 414 (100 mg, 0.26 mmol), TFA (1.6 mL), $\mathrm{CHCl}_{3}$ $(1.6 \mathrm{~mL})$ and formaline $(37 \%, 0.25 \mathrm{~mL})$ was stirred for $30 \mathrm{~min}$ at r.t. Afterwards, triethylsilane $(0.6 \mathrm{~mL}, 5.2 \mathrm{mmol})$ was added carefully with cooling. The resulting mixture was stirred for $30 \mathrm{~min}$ at r.t., and then all volatiles were evaporated in vacuo. The residue was subjected to column chromatography (50 g of $\mathrm{SiO}_{2}, \mathrm{CH}_{2} \mathrm{Cl}_{2} / \mathrm{MeOH}, 5: 1 \rightarrow 3: 1$ ) to furnish $100 \mathrm{mg}$ (96\%) of the title compound. ${ }^{1} \mathrm{H}$ NMR (300 MHz, $\mathrm{CD}_{3} \mathrm{OD}$ ): $\delta=2.81$ (s, 3 H, NMe), 3.39-3.49 (m, 2 H, $\mathrm{CH}_{2}$ ), 3.50-3.55 (m, 2 H, $\left.\mathrm{CH}_{2}\right), 3.55-3.62$ (m, $4 \mathrm{H}, 2 \times$ $\mathrm{CH}_{2}$ ), 3.92 (s, $2 \mathrm{H}, \mathrm{CH}_{2}$ ), 4.12 (s, $2 \mathrm{H}, \mathrm{CH}_{2}$ ), 7.25-7.31 (m, 2H, Ar), 7.34-7.40 (m, 2H, Ar), 7.49 (d, $J=7.4$ Hz, 2 H, Ar), 7.79 (d, $J=7.5$ Hz, 2 H, Ar) ppm. MS (ESI): $m / z=422$ (100) $[\mathrm{M}+\mathrm{Na}]^{+}$.

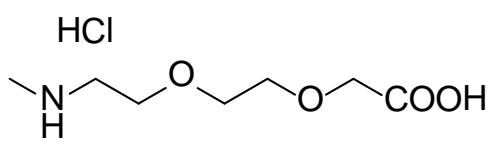

\section{2-(2-(2- $N$-Methylaminoethoxy)ethoxy)acetic acid hydrochloride $(415-\mathrm{H} \cdot \mathrm{HCl})$}

Compound 415-Fmoc (50 mg; 0.125 mmol) was dissolved in a 30\% solution of piperidine in DMF (2 ml). The resulting mixture was stirred for $30 \mathrm{~min}$ at r.t., and then, all volatiles were evaporated in vacuo. The residue was dissolved in diluted aq. $\mathrm{HCl}(20 \mathrm{ml})$, and this solution was extracted with $\mathrm{Et}_{2} \mathrm{O}(3 \times 20 \mathrm{~mL})$. The aq. phase was evaporated, and the residue was subjected to column chromatography (25 g of $\mathrm{SiO}_{2}$, $\left.\mathrm{CHCl}_{3} / \mathrm{MeOH} / \mathrm{H}_{2} \mathrm{O} / \mathrm{HCOOH}, 75: 25: 5: 1\right)$ to furnish $20 \mathrm{mg}$ (74\%) of the title compound. ${ }^{1} \mathrm{H}$ NMR (300 MHz, CD $\mathrm{CD}$ ): $\delta=2.38$ (s, $3 \mathrm{H}, \mathrm{NMe}$ ), 2.71-2.76 (m, $2 \mathrm{H}, \mathrm{CH}_{2}$ ), 3.553.60 (m, 2 H, $\mathrm{CH}_{2}$ ), 3.63 (s, $4 \mathrm{H}, 2 \times \mathrm{CH}_{2}$ ), 3.87 (s, $2 \mathrm{H}, \mathrm{CH}_{2}$ ), 8.52 (s, $\left.1 \mathrm{H}, \mathrm{NH}\right) \mathrm{ppm} .{ }^{13} \mathrm{C}$ NMR (75.5 MHz, $\left.\mathrm{CD}_{3} \mathrm{OD}\right): \delta=34.4(+)$, 50.1(-), 68.9(-), 69.3(-), 69.5(-), 70.2(-), 176.3(-) ppm. MS (ESI): m/z (positive mode, rel. int., \%) = $178(100)[\mathrm{M}+\mathrm{H}]^{+}$.<smiles>CN(CCOCCOCC(=O)ON1C(=O)CCC1=O)C(=O)c1ccccc1C1=C2C=CC(=O)C=C2C(C)(C)c2cc(N(C)C)ccc21</smiles> 
2,5-Dioxopyrrolidin-1-yl 2-(2-(2-(2-(6- $N, N$-Dimethylamino)-10,10-dimethyl-3-oxo3,10-dihydroanthracen-9-yl)- $N$ '-methylbenzamido)ethoxy)ethoxy)acetate

(416NHS)

To a stirred solution of compound 416-H ( $3 \mathrm{mg}, 5.5 \mu \mathrm{mol})$ and $N$-hydroxysuccinimide $(0.8 \mathrm{mg}, 6.6 \mu \mathrm{mol})$ in MeCN (1 mL), HATU (3.1 mg, $8.2 \mu \mathrm{mol})$ was added followed by $\mathrm{NEt}_{3}(5 \mu \mathrm{L}, 33 \mu \mathrm{mol})$. The reaction mixture was stirred overnight and then, evaporated in vacuo. The residue was subjected to flash chromatography on $\mathrm{SiO}_{2}$ (5 g, $\mathrm{MeCN} / \mathrm{H}_{2} \mathrm{O}$, 10:1). The main fraction was filtered through a fine glass filter and freeze-dried to give $1.6 \mathrm{mg}$ (45\%) of the title compound. HPLC: $\mathrm{B} / \mathrm{A}=30 / 70$ to $100 / 0$ in $25 \mathrm{~min}$, detection at $550 \mathrm{~nm}, t_{\mathrm{R}}=9.6 \mathrm{~min}(45 \%)$. MS (ESI): $\mathrm{m} / \mathrm{z}$ (positive mode, rel. int., \%) $=642(100)$ $[\mathrm{M}+\mathrm{Na}]^{+}$.<smiles>CN(C)C(=O)c1ccc(N(C)C)cc1</smiles>

\section{4- $N, N$-Dimethylamino- $N$ '-methoxy- $N$ '-methylbenzamide (418)}

Compound 418 was prepared according to the known procedure: ${ }^{[177]}$ To a suspension of 4- $N, N$-dimethylaminobenzoic acid $(1000 \mathrm{mg}, 6 \mathrm{mmol})$ in $\mathrm{CH}_{2} \mathrm{Cl}_{2}(30 \mathrm{~mL})$ triphosgene (891 mg, $3 \mathrm{mmol}$ ) and $\mathrm{NEt}_{3}\left(4.3 \mathrm{~mL}, 30 \mathrm{mmol}\right.$ ) were added at $0{ }^{\circ} \mathrm{C}$ under Ar. To the resulting blue solution, $\mathrm{N}, \mathrm{O}$-dimethylhydroxylamine hydrochloride (588 mg, $6 \mathrm{mmol}$ ) was added. The reaction mixture changed its color from blue to yellow, and a precipitate of $\mathrm{NEt}_{3} \cdot \mathrm{HCl}$ formed. The reaction mixture was allowed to warm up to r.t., and then was stirred for $3 \mathrm{~h}$. The precipitate was filtered off, and the filtrate was shaken with water (20 $\mathrm{mL})$. The organic layer was separated, and the aq. layer was extracted with $\mathrm{CH}_{2} \mathrm{Cl}_{2}(3 \times 20$ $\mathrm{mL}$ ). Combined organic solutions were dried with $\mathrm{Na}_{2} \mathrm{SO}_{4}$ and evaporated. The residue was subjected to column chromatography (30 g of $\mathrm{SiO}_{2}, n$-hexane/EtOAc, 2:3) to yield $1228 \mathrm{mg}$ (98\%) of the title compound. ${ }^{1} \mathrm{H}$ NMR (300 MHz, $\left.\mathrm{CDCl}_{3}\right): \delta=3.02$ (s, $6 \mathrm{H}$, $\mathrm{NMe}_{2}$ ), 3.35 (s, 3 H, NMe), 3.59 (s, 3 H, OMe), 6.60-6.71 (m, 2 H, Ar), 7.66-7.77 (m, 2 H, Ar) ppm. MS (ESI): m/z (positive mode, rel. int., \%) = $209(41)[\mathrm{M}+\mathrm{H}]^{+}, 231(100)$ $[\mathrm{M}+\mathrm{Na}]^{+}$.<smiles>COc1c(F)c(F)c(Br)c(Br)c1F</smiles>

1,2-Dibromo-3,4,6-trifluoro-5-methoxybenzene (420) 
$\mathrm{Na}$ (336 mg, $14.6 \mathrm{mmol}$ ) was dissolved in $\mathrm{MeOH}(30 \mathrm{~mL}$ ) carefully, and to the resulting solution, 1,2-dibromo-3,4,5,6-tetrafluorobenzene (4000 mg, $13.3 \mathrm{mmol}$ ) was added. The reaction mixture was refluxed for 6 h, cooled down to r.t., and then, poured in water $(100 \mathrm{~mL})$. The resulting slurry was extracted with $\mathrm{Et}_{2} \mathrm{O}(3 \times 70 \mathrm{~mL})$. Combined organic solutions were dried with $\mathrm{Na}_{2} \mathrm{SO}_{4}$ and evaporated in vacuo to give $4240 \mathrm{mg}$ (99\%) of the crude product which was used without further purification. ${ }^{1} \mathrm{H}$ NMR $\left(300 \mathrm{MHz}, \mathrm{CDCl}_{3}\right)$ : $\delta=4.04$ (m, $3 \mathrm{H}, \mathrm{OMe}$ ) ppm. ${ }^{19} \mathrm{~F}$ NMR (282.2 MHz, $\left.\mathrm{CDCl}_{3}\right): \delta=-149.26$ (dd, $J=21.9$ and $5.4 \mathrm{~Hz}, 1 \mathrm{~F}$ ), -126.52 (dd, $J=21.6$ and $8.2 \mathrm{~Hz}, 1 \mathrm{~F}$ ), -119.28 (m, 1 F) ppm.<smiles>COc1c(F)c(F)cc(Br)c1F</smiles>

\section{1-Bromo-2,4,5-trifluoro-3-methoxybenzene (421)}

Compound 421 was prepared according to the known procedure: ${ }^{[168 b]}$ In a $50 \mathrm{~mL}$ Schlenk flask to a solution of compound 420 (2000 mg, $6.25 \mathrm{mmol})$ in $\mathrm{Et}_{2} \mathrm{O}(20 \mathrm{~mL}), 1.6 \mathrm{M}$ solution of BuLi in hexanes $(4.5 \mathrm{~mL}, 7.18 \mathrm{mmol})$ was injected carefully at $-78{ }^{\circ} \mathrm{C}$. After $4 \mathrm{~h}$ stirring at this temperature, the reaction mixture was quenched with $50 \%$ aq. EtOH (20 $\mathrm{mL})$. The organic layer was separated, and the aq. layer was extracted with $\mathrm{Et}_{2} \mathrm{O}(2 \times 50$ $\mathrm{mL}$ ). Combined organic solutions were dried with $\mathrm{Na}_{2} \mathrm{SO}_{4}$ and evaporated. The residue was purified by column chromatography (70 g of $\mathrm{SiO}_{2}, n$-hexane) to yield $855 \mathrm{mg}$ (57\%) of a colorless oil. ${ }^{1} \mathrm{H}$ NMR (300 MHz, $\mathrm{CDCl}_{3}$ ): $\delta=4.03$ (m, $3 \mathrm{H}, \mathrm{OMe}$ ), 7.04-7.13 (m, 1 $\mathrm{H}$, Ar) ppm. ${ }^{19} \mathrm{~F}$ NMR (282.2 MHz, $\left.\mathrm{CDCl}_{3}\right): \delta=-151.31$ (m, $\left.1 \mathrm{~F}\right),-139.63$ (m, $1 \mathrm{~F}$ ), $-125.67(\mathrm{~m}, 1 \mathrm{~F}) \mathrm{ppm}$.<smiles>COc1c(F)cc(C(=O)c2ccc(N(C)C)cc2)c(F)c1F</smiles>

\section{(4- $N, N$-dimethylaminophenyl)(2,3,5-trifluoro-4-methoxyphenyl)methanone (422)}

To a solution of compound 420 (1000 mg, $3.12 \mathrm{mmol})$ in THF (10 mL), 1.6 M solution of BuLi in hexanes $(2.25 \mathrm{~mL}, 3.6 \mathrm{mmol})$ was injected at $-78{ }^{\circ} \mathrm{C}$. After $30 \mathrm{~min}$ stirring, a solution of compound 418 (844 mg, $4.07 \mathrm{mmol}$ ) in THF (5 mL) was added, the resulting solution was stirred for $30 \mathrm{~min}$ at $-78{ }^{\circ} \mathrm{C}$, for $10 \mathrm{~min}$ at $0{ }^{\circ} \mathrm{C}$, and then, quenched with sat. aq. $\mathrm{NH}_{4} \mathrm{Cl}(5 \mathrm{~mL})$. The organic layer was separated, and the aq. layer was extracted with 
EtOAc (3×20). Combined organic solutions were dried with $\mathrm{Na}_{2} \mathrm{SO}_{4}$ and evaporated. The crude product was purified by column chromatography (100 g of $\mathrm{SiO}_{2}, n$-hexane/EtOAc, 4:1) to furnish $270 \mathrm{mg}$ (28\%) as a yellow oil. ${ }^{1} \mathrm{H} \mathrm{NMR}$ (300 $\mathrm{MHz}, \mathrm{CDCl}_{3}$ ): $\delta=3.07$ (s, 6 H, $\mathrm{NMe}_{2}$ ), 4.09 (m, 3 H, OMe), 6.61-6.67 (m, 2 H, Ar), 7.03 (ddd, $J=10.7,5.6$ and 2.4 $\mathrm{Hz}, 1 \mathrm{H}$, Ar) 7.69-7.73 (m, $2 \mathrm{H}, \mathrm{Ar}$ ) ppm. ${ }^{19} \mathrm{~F}$ NMR (282.2 MHz, $\mathrm{CDCl}_{3}$ ): $\delta=-150.62$ (m, $1 \mathrm{~F}),-139.33$ (m, $1 \mathrm{~F}),-132.96$ (m, $1 \mathrm{~F}) \mathrm{ppm} .{ }^{13} \mathrm{C}$ NMR (125.7 MHz, $\left.\mathrm{CDCl}_{3}\right): \delta=$ 40.0(+), 61.9(+, t, $J=4 \mathrm{~Hz}), 110.6(+), 111.2(+, \mathrm{dt}, \mathrm{J}=22$ and $4 \mathrm{~Hz}), 122.4(-, \mathrm{d}, J=15$ Hz), 124.0(-), 132.3(+), 139.1(-, m), 143.9(-, ddd, $J=$ 251, 16 and 6 Hz), 145.1(-, ddd, $J$ = 249, 12 and $3 \mathrm{~Hz}), 150.6(-, \mathrm{dt}, J=246$ and $3 \mathrm{~Hz}), 153.8(-), 187.8(-)$ ppm. HRMS (ESI): found 310.1044; calc. for $\mathrm{C}_{16} \mathrm{H}_{14} \mathrm{NO}_{2} \mathrm{~F}_{3}[\mathrm{M}+\mathrm{H}]^{+} 310.1049$.<smiles>COc1c(F)c(F)c(C(=O)O)c(Br)c1F</smiles>

\section{2-Bromo-3,5,6-trifluoro-4-methoxybenzoic acid (423)}

To a solution of compound 420 (100 mg, 0.31 mmol) in $\mathrm{Et}_{2} \mathrm{O}(1 \mathrm{~mL}), 1.6 \mathrm{M}$ solution of BuLi in hexanes $(193 \mu \mathrm{L}, 0.31 \mathrm{mmol})$ was injected over $7 \mathrm{~min}$ at $-78{ }^{\circ} \mathrm{C}$. After $30 \mathrm{~min}$ stirring at this temperature, an excess of solid $\mathrm{CO}_{2}$ was added. The reaction mixture was allowed to reach r.t., and $1 \mathrm{M} \mathrm{HCl}$ was added $(0.5 \mathrm{~mL})$. The resulting slurry was extracted with $\mathrm{CH}_{2} \mathrm{Cl}_{2}(3 \times 5 \mathrm{~mL})$. Combined organic solutions were dried with $\mathrm{Na}_{2} \mathrm{SO}_{4}$ and evaporated in vacuo. The crude product was subjected to column chromatography (15 g of $\left.\mathrm{SiO}_{2}, \mathrm{CH}_{2} \mathrm{Cl}_{2} / \mathrm{MeOH}, 4: 1\right)$ to afford $71 \mathrm{mg}(81 \%)$ of the title product. ${ }^{1} \mathrm{H}$ NMR (300 $\mathrm{MHz}, \mathrm{CDCl}_{3}$ ): $\delta=4.12$ (m, $3 \mathrm{H}, \mathrm{OMe}$ ) ppm. ${ }^{19} \mathrm{~F}$ NMR (282.2 MHz, $\left.\mathrm{CDCl}_{3}\right): \delta=-150.3$ (m, 1 F), -137.7 (m, 1 F), -122.1 (m, 1 F) ppm. MS (ESI): m/z (negative mode, rel. int., $\%)=239(100)\left[\mathrm{M}-\mathrm{COOH},{ }^{79} \mathrm{Br}\right]^{-}, 241(96)\left[\mathrm{M}-\mathrm{COOH},{ }^{81} \mathrm{Br}\right]^{-}$.<smiles>COc1c(F)c(F)c(C(O)c2ccc(N(C)C)cc2)c(Br)c1F</smiles>

\section{(2-Bromo-3,5,6-trifluoro-4-methoxyphenyl)(4- $N, N$-dimethylaminophenyl)methanol}

To a solution of compound 420 (320 mg, $1 \mathrm{mmol}$ ) in THF (3 mL), 1.6 M BuLi in hexanes was added dropwise at $-78{ }^{\circ} \mathrm{C}$. After 30 min stirring at this temperature, a solution of 4- 
$\mathrm{N}, \mathrm{N}$-dimethylaminobenzaldehyde in $\mathrm{Et}_{2} \mathrm{O}(5 \mathrm{~mL})$ was injected. The resulted mixture was stirred for $30 \mathrm{~min}$ at $-78{ }^{\circ} \mathrm{C}$ and then, allowed to warm to r.t. Afterwards, sat. aq. $\mathrm{NH}_{4} \mathrm{Cl}$ (2 mL), water (5 mL) and $\mathrm{CH}_{2} \mathrm{Cl}_{2}(5 \mathrm{~mL})$ were added. The organic layer was separated, and the aq. layer was extracted with $\mathrm{CH}_{2} \mathrm{Cl}_{2}(3 \times 10 \mathrm{~mL})$. Combined organic solutions were dried with $\mathrm{Na}_{2} \mathrm{SO}_{4}$ and evaporated. The crude product was purified by column chromatography (30 g of $\mathrm{SiO}_{2}, n$-hexane/EtOAc, 2:1) to furnish $336 \mathrm{mg}$ (86\%) of a yellow oil. ${ }^{1} \mathrm{H}$ NMR (300 MHz, $\mathrm{CDCl}_{3}$ ): $\delta=2.73$ (dd, $J=8.9$ and $2.9 \mathrm{~Hz}, 1 \mathrm{H}$ ), $2.93(\mathrm{~s}, 6 \mathrm{H}$, $\mathrm{NMe}_{2}$ ), 4.03 (t, $J=1.2 \mathrm{~Hz}, 3 \mathrm{H}, \mathrm{OMe}$ ), 6.24 (br. d, $J=8.6$ Hz, $1 \mathrm{H}, \mathrm{OH}$ ), 6.65-6.70 (m, 2 H, Ar), 7.20 (m, 2 H, Ar) ppm. ${ }^{19} \mathrm{~F} \mathrm{NMR} \mathrm{(282.2} \mathrm{MHz,} \mathrm{CDCl}_{3}$ ): $\delta=-151.15$ (dd, $J=20.2$ and $5.5 \mathrm{~Hz}, 1 \mathrm{~F}$ ), -142.06 (dd, $J=20.1$ and $9.3 \mathrm{~Hz}, 1 \mathrm{~F}$ ), -122.97 (m, $1 \mathrm{~F}$ ) ppm. MS (ESI): $m / z$ (positive mode, rel. int., \%) = $390(100)\left[\mathrm{M}+\mathrm{H},{ }^{79} \mathrm{Br}\right]^{+}, 392(91)\left[\mathrm{M}+\mathrm{H},{ }^{81} \mathrm{Br}\right]^{+}$.<smiles>COc1c(F)c(F)c(C(=O)N(C)OC)c(Br)c1F</smiles>

\section{2-Bromo-3,5,6-trifluoro- $N$,4-dimethoxy- $N$-methylbenzamide (425)}

To a suspension of compound 423 (1000 mg, $3.5 \mathrm{mmol})$ in $\mathrm{CH}_{2} \mathrm{Cl}_{2}$ (30 mL) triphosgene (727 mg, $2.45 \mathrm{mmol})$ and $\mathrm{NEt}_{3}(2.5 \mathrm{~mL}, 17.5 \mathrm{mmol})$ were added at $0{ }^{\circ} \mathrm{C}$ under Ar. To the resulting solution, $\mathrm{N}, \mathrm{O}$-dimethylhydroxylamine hydrochloride (378 $\mathrm{mg}, 3.8 \mathrm{mmol}$ ) was added. The reaction mixture was allowed to warm up to r.t., and then was stirred for 1.5 h. The precipitate of $\mathrm{NEt}_{3} \cdot \mathrm{HCl}$ was filtered off, and the filtrate was shaken with water (20 $\mathrm{mL})$. The organic layer was separated, and the aq. layer was extracted with $\mathrm{CH}_{2} \mathrm{Cl}_{2}(3 \times 20$ $\mathrm{mL}$ ). Combined organic solutions were dried with $\mathrm{Na}_{2} \mathrm{SO}_{4}$ and evaporated. The residue was subjected to column chromatography (30 g of $\mathrm{SiO}_{2}, n$-hexane/EtOAc, 3:1) to yield $741 \mathrm{mg}(65 \%)$ of the title compound. ${ }^{1} \mathrm{H}$ NMR (300 MHz, $\left.\mathrm{CDCl}_{3}\right): \delta=3.38$ (s, $3 \mathrm{H}$, NMe), 3.55 (s, 3 H, OMe), 4.06 (t, J = $1.3 \mathrm{~Hz}, 3 \mathrm{H}$, OMe) ppm. ${ }^{19} \mathrm{~F}$ NMR (282.2 MHz, $\mathrm{CDCl}_{3}$ ): $\delta=-150.61$ (ddd, $J=21.6,6.5$ and $1.3 \mathrm{~Hz}, 1 \mathrm{~F}$ ), -140.60 (dd, $J=21.6$ and 10.2 $\mathrm{Hz}, 1 \mathrm{~F}$ ), -124.10 (ddd, $J=10.0,6.5$ and $1.3 \mathrm{~Hz}, 1 \mathrm{~F}$ ) ppm. HRMS (ESI): found 327.9792; calc. for $\mathrm{C}_{10} \mathrm{H}_{9} \mathrm{NO}_{3} \mathrm{~F}_{3} \mathrm{Br}[\mathrm{M}+\mathrm{H}]^{+} 327.9791$.<smiles>COc1c(F)c(F)c(Cc2ccc(N(C)C)cc2)c(Br)c1F</smiles> 


\section{4-(2-Bromo-3,5,6-trifluoro-4-methoxybenzyl)- $N, N$-dimethylaniline (426)}

Compound 424 (200 mg, $0.51 \mathrm{mmol}$ ) was dissolved in a $\mathrm{CH}_{2} \mathrm{Cl}_{2} / \mathrm{TFA}$ mixture (2:5, $7 \mathrm{ml}$ ), and to the resulting solution, $\mathrm{Et}_{3} \mathrm{SiH}(1.5 \mathrm{~mL}, \sim 9.8 \mathrm{mmol})$ was added. The reaction mixture was stirred overnight at r.t. and $7 \mathrm{~h}$ at $40{ }^{\circ} \mathrm{C}$. Afterwards, all volatiles were evaporated, the residue was dissolved in $\mathrm{CH}_{2} \mathrm{Cl}_{2}(\sim 10 \mathrm{~mL})$, the resulting solution was washed with sat. aq. $\mathrm{NaHCO}_{3}$, dried with $\mathrm{Na}_{2} \mathrm{SO}_{4}$, and evaporated to dryness. The crude product was purified by column chromatography (30 g of $\mathrm{SiO}_{2}, n$-hexane/Et $2 \mathrm{O}, 10: 1$ ) to furnish $187 \mathrm{mg}(97 \%)$ of the title compound. ${ }^{1} \mathrm{H}$ NMR (300 MHz, $\left.\mathrm{CDCl}_{3}\right): \delta=2.91(\mathrm{~s}, 6 \mathrm{H}$, $\mathrm{NMe}_{2}$ ), 4.01 (t, $J=1.1 \mathrm{~Hz}, 3 \mathrm{H}, \mathrm{OMe}$ ), 4.07 (d, $J=2.8 \mathrm{~Hz}, 2 \mathrm{H}, \mathrm{CH}_{2}$ ), 6.64-6.68 (m, $2 \mathrm{H}$, Ar), 7.12-7.17 (m, $2 \mathrm{H}$, Ar) ppm. ${ }^{19} \mathrm{~F}$ NMR (282.2 MHz, $\left.\mathrm{CDCl}_{3}\right): \delta=-151.98$ (dd, $J=$ 20.9 and $4.8 \mathrm{~Hz}, 1 \mathrm{~F}$ ), -140.63 (ddt, $J=20.6,10.2$ and $2.7 \mathrm{~Hz}, 1 \mathrm{~F}$ ), -123.29 (dd, $J=10.2$ and $4.7 \mathrm{~Hz}, 1 \mathrm{~F}$ ) ppm. MS (ESI): m/z (positive mode, rel. int., \%) = 374 (100) $[\mathrm{M}+\mathrm{H}$, $\left.{ }^{79} \mathrm{Br}\right]^{+}, 376(94)\left[\mathrm{M}+\mathrm{H},{ }^{81} \mathrm{Br}\right]^{+}$.<smiles>COc1c(F)c(F)c2c(=O)n(OC)cc(C)c2c1F</smiles>

\section{2,3,5-Trifluoro- $N$,4-dimethoxy- $N$-methyl-6-(prop-1-en-2-yl)benzamide (427)}

In a screw-cap tube compound 297 (200 mg, $0.6 \mathrm{mmol}$ ), isopropenylboronic acid (62 mg, $0.72 \mathrm{mmol}$ ), $\mathrm{Pd}\left(\mathrm{PPh}_{3}\right)_{4}$ (35 mg, $\left.0.03 \mathrm{mmol}, 5 \mathrm{~mol} \%\right), 2 \mathrm{M} \mathrm{Na}_{2} \mathrm{CO}_{3}(1.2 \mathrm{~mL}, 2.4 \mathrm{mmol}$ ), EtOH $(1.2 \mathrm{~mL})$ and toluene $(2 \mathrm{~mL})$ were placed under argon. The mixture was heated to $110^{\circ} \mathrm{C}$ and left overnight at this temperature with stirring. Afterwards, the reaction mixture was allowed to cool to r.t., diluted with $\mathrm{CH}_{2} \mathrm{Cl}_{2}(10 \mathrm{~mL})$, passed through a plug of Celite (eluting with $\mathrm{CH}_{2} \mathrm{Cl}_{2}$ ), and the filtrate was evaporated in vacuo. Column chromatography (30 g of $\mathrm{SiO}_{2}, n$-hexane/EtOAc, 4:1) furnished the title product as a yellow oil (110 mg, 64\%). ${ }^{1} \mathrm{H}$ NMR (300 MHz, $\mathrm{CDCl}_{3}$ ): $\delta=2.03$ (s, $3 \mathrm{H}, \mathrm{Me}$ ), 3.27 (s, $3 \mathrm{H}, \mathrm{NMe}$ ), 3.55 (s, $3 \mathrm{H}, \mathrm{OMe}$ ), 4.04 (t, J = $1.3 \mathrm{~Hz}, 3 \mathrm{H}, \mathrm{OMe}$ ), 4.98 (s, $1 \mathrm{H}), 5.22$ (m, 1H) ppm. ${ }^{19} \mathrm{~F}$ NMR (282.2 MHz, $\mathrm{CDCl}_{3}$ ): $\delta=-152.83$ (m, $1 \mathrm{~F}$ ), -143.26 (dd, $J=21.7$ and $12.3 \mathrm{~Hz}$, $1 \mathrm{~F}$ ), -134.81 (dd, $J=12.2$ and $5.6 \mathrm{~Hz}, 1 \mathrm{~F}$ ) ppm. HRMS (ESI): found 290.1002; calc. for $\mathrm{C}_{13} \mathrm{H}_{14} \mathrm{~F}_{3} \mathrm{NO}_{3}[\mathrm{M}+\mathrm{H}]^{+} 290.0999$. 


\section{Acknowledgments}

Success of my doctoral project would have been impossible without inspiration, support and guidance from many people.

I would like to express my deepest gratitude to Prof. Stefan Hell who provided me an excellent opportunity to pursue my doctoral studies in a competitive and interdisciplinary environment of Department of NanoBiophotonics at MPI BPC. I am very thankful to Prof. Armin de Meijere for his careful reading of my yearly progress reports, giving me important feedback and his help in thesis preparation. A very special thanks goes to Dr. Vladimir N. Belov for suggesting the interesting topic of my doctoral studies, his great supervision and encouragement throughout the whole time of my stay at MPI BPC.

I would like to extend my thanks to all members of Organic Synthesis group in the NanoBiophotonics department for the wonderful working atmosphere, support and critical discussions. In particular, I am very thankful to Dr. Kirill Kolmakov and Dr. Shamil Nizamov for sharing their knowledge and rich experience in the synthesis of organic fluorophores. I am especially grateful to Dr. Heiko Schill for his readiness in teaching me how to work with absorption and fluorescence spectrometers and also for writing an Origin script that saved an enormous amount of time on data processing. A separate thanks goes to Dr. Matthias Bischoff who provided important synthetic intermediates.

I greatly appreciate assistance and commitment from the colleagues in Department of NanoBiophotonics without whose input the completion of this project would not have been possible. I would like to thank Dr. Christian Wurm, Dr. Katrin I. Willig and Elke Hebisch for recording numerous super-resolution images. I am also very thankful to Prof. Mariano L. Bossi, Nina Ohm, Marianne Pulst and Kurt Müller for their help in measuring important photophysical properties.

Finally, I would like to express the appreciation to Prof. Ulf Diederichsen, Prof. Claudia Höbartner, Prof. Konrad Koszinowski and Prof. Heinz Neumann for their readiness to serve as members of the examination board on my $\mathrm{PhD}$ defence. Thank you. 


\section{References}

[1] S. W. Hell, Science 2007, 316, 1153-1158.

[2] B. Valeur, in Molecular Fluorescence, Wiley-VCH, 2001, pp. 34-71.

[3] S. A. Adam, Genome Biol. 2001, 2, Reviews0007.

[4] S. W. Hell, J. Wichmann, Opt. Lett. 1994, 19, 780-782.

[5] S. W. Hell, Nat. Biotechnol. 2003, 21, 1347-1355.

[6] S. W. Hell, M. Kroug, Appl. Phys. B: Lasers Opt. 1995, 60, 495-497.

[7] S. Bretschneider, C. Eggeling, S. W. Hell, Phys. Rev. Lett. 2007, 98, 218103.

[8] M. Hofmann, C. Eggeling, S. Jakobs, S. W. Hell, Proc. Natl. Acad. Sci. U.S.A. 2005, 102, 17565-17569.

[9] F. Lavoie-Cardinal, N. A. Jensen, V. Westphal, A. C. Stiel, A. Chmyrov, J. Bierwagen, I. Testa, S. Jakobs, S. W. Hell, ChemPhysChem 2014, 15, 655-663.

[10] a) L. Song, E. A. Jares-Erijman, T. M. Jovin, J. Photochem. Photobiol. A 2002, 150, 177-185; b) L. Giordano, T. M. Jovin, M. Irie, E. A. Jares-Erijman, J. Am. Chem. Soc. 2002, 124, 7481-7489.

[11] M. Bossi, J. Fölling, M. Dyba, V. Westphal, S. W. Hell, New J. Phys. 2006, 8, 275.

[12] a) E. Betzig, G. H. Patterson, R. Sougrat, O. W. Lindwasser, S. Olenych, J. S. Bonifacino, M. W. Davidson, J. Lippincott-Schwartz, H. F. Hess, Science 2006, 313, 1642-1645; b) S. T. Hess, T. P. K. Girirajan, M. D. Mason, Biophys. J. 2006, 91, 4258-4272.

[13] M. J. Rust, M. Bates, X. Zhuang, Nat. Methods 2006, 3, 793-796.

[14] M. Heilemann, S. van de Linde, M. Schüttpelz, R. Kasper, B. Seefeldt, A. Mukherjee, P. Tinnefeld, M. Sauer, Angew. Chem. Int. Ed. 2008, 47, 6172-6176.

[15] J. Fölling, M. Bossi, H. Bock, R. Medda, C. A. Wurm, B. Hein, S. Jakobs, C. Eggeling, S. W. Hell, Nat. Methods 2008, 5, 943-945.

[16] V. Westphal, S. O. Rizzoli, M. A. Lauterbach, D. Kamin, R. Jahn, S. W. Hell, Science 2008, 320, 246-249.

[17] A. Chmyrov, J. Keller, T. Grotjohann, M. Ratz, E. d'Este, S. Jakobs, C. Eggeling, S. W. Hell, Nat. Methods 2013, 10, 737-740.

[18] G. Donnert, J. Keller, C. A. Wurm, S. O. Rizzoli, V. Westphal, A. Schönle, R. Jahn, S. Jakobs, C. Eggeling, S. W. Hell, Biophys. J. 2007, 92, L67-L69. 
[19] R. Schmidt, C. A. Wurm, S. Jakobs, J. Engelhardt, A. Egner, S. W. Hell, Nat. Methods 2008, 5, 539-544.

[20] X. Liu, Z. Xu, J. M. Cole, J. Phys. Chem. C 2013, 117, 16584-16595.

[21] a) G. A. Reynolds, K. H. Drexhage, Opt. Commun. 1975, 13, 222-225; b) K. H. Drexhage, G. R. Erikson, G. H. Hawks, G. A. Reynolds, Opt. Commun. 1975, 15, 399-403; c) A. N. Fletcher, D. E. Bliss, Appl. Phys. 1978, 16, 289-295; d) G. Jones II, W. R. Jackson, A. M. Halpern, Chem. Phys. Lett. 1980, 72, 391-395; e) G. Jones Ii, W. R. Jackson, S. Kanoktanaporn, A. M. Halpern, Opt. Commun. 1980, 33, 315-320; f) A. N. Fletcher, D. E. Bliss, J. M. Kauffman, Opt. Commun. 1983, 47, 57-61; g) G. Jones, W. R. Jackson, C. Y. Choi, W. R. Bergmark, J. Phys. Chem. 1985, 89, 294-300.

[22] W.-C. Sun, K. R. Gee, R. P. Haugland, Bioorg. Med. Chem. Lett. 1998, 8, 31073110 .

[23] X. Jin, C. Uttamapinant, A. Y. Ting, ChemBioChem 2011, 12, 65-70.

[24] D. P. Specht, P. A. Martic, S. Farid, Tetrahedron 1982, 38, 1203-1211.

[25] a) E. Koller, O. S. Wolfbeis, Monatsh. Chem. 1985, 116, 65-75; b) O. S. Wolfbeis, E. Koller, P. Hoghmuth, Bull. Chem. Soc. Jpn. 1985, 58, 731-734.

[26] G. Signore, R. Nifosi, L. Albertazzi, B. Storti, R. Bizzarri, J. Am. Chem. Soc. 2010, 132, 1276-1288.

[27] T. Deligeorgiev, T. Tsvetkova, S. Stanimirov, Color. Technol. 2011, 127, 434439.

[28] D. H. McDaniel, H. C. Brown, J. Org. Chem. 1958, 23, 420-427.

[29] O. S. Wolfbeis, H. Marhold, Chem. Ber. 1985, 118, 3664-3672.

[30] P. Czerney, M. Wenzel, B. Schweder, F. Lehmann, EP 1318177 B1; US 7563907 B2.

[31] J.-A. Richard, M. Massonneau, P.-Y. Renard, A. Romieu, Org. Lett. 2008, 10, 4175-4178.

[32] a) P. Kele, X. Li, M. Link, K. Nagy, A. Herner, K. Lorincz, S. Beni, O. S. Wolfbeis, Org. Biomol. Chem. 2009, 7, 3486-3490; b) T. Ehrenschwender, B. R. Varga, P. Kele, H.-A. Wagenknecht, Chem. Asian J. 2010, 5, 1761-1764; c) K. Nagy, E. Orban, S. Bosze, P. Kele, Chem. Asian J. 2010, 5, 773-777; d) G. B. Cserep, K. N. Enyedi, A. Demeter, G. Mezo, P. Kele, Chem. Asian J. 2013, 8, 494-502. 
[33] I. I. Grandberg, L. K. Denisov, O. A. Popova, Chem. Heterocycl. Compd. 1987, 23, 117-142.

[34] T. Hinohara, K. Amano, K. Matsui, Nippon Kagaku Kaishi 1976, 1976, 247-247.

[35] K. Rechthaler, G. Köhler, Chem. Phys. 1994, 189, 99-116.

[36] M. Weißenfels, A. Hantschmann, T. Steinführer, E. Birkner, Z. Chem. 1989, 29, $166-166$.

[37] L. A. Karandashova, M. A. Kirpichenok, D. S. Yufit, Y. T. Struchkov, I. I. Grandberg, Chem. Heterocycl. Compd. 1990, 26, 1338-1345.

[38] N. A. Gordeeva, M. A. Kirpichenok, N. S. Patalakha, I. I. Grandberg, Chem. Heterocycl. Compd. 1990, 26, 1329-1337.

[39] N. A. Gordeeva, M. A. Kirpichenok, N. S. Patalakha, V. M. Khutoretskii, I. I. Grandberg, Chem. Heterocycl. Compd. 1991, 27, 491-495.

[40] M. A. Kirpichenok, V. M. Baukulev, L. A. Karandashova, I. I. Grandberg, Chem. Heterocycl. Compd. 1991, 27, 1193-1199.

[41] M. A. Kirpichenok, N. S. Patalakha, L. Y. Fomina, I. I. Grandberg, Chem. Heterocycl. Compd. 1991, 27, 934-939.

[42] N. S. Patalakha, D. S. Yufit, M. A. Kirpichenok, N. A. Gordeeva, Y. T. Struchkov, I. I. Grandberg, Chem. Heterocycl. Compd. 1991, 27, 32-37.

[43] J. Gordo, J. Avó, A. J. Parola, J. C. Lima, A. Pereira, P. S. Branco, Org. Lett. 2011, 13, 5112-5115.

[44] H. Takechi, Y. Oda, N. Nishizono, K. Oda, M. Machida, Chem. Pharm. Bull. 2000, 48, 1702-1710.

[45] a) S. Nizamov, K. I. Willig, M. V. Sednev, V. N. Belov, S. W. Hell, Chem. Eur. J. 2012, 18, 16339-16348; b) H. Schill, S. Nizamov, F. Bottanelli, J. Bierwagen, V. N. Belov, S. W. Hell, Chem. Eur. J. 2013, 19, 16556-16565.

[46] M.-S. Schiedel, C. A. Briehn, P. Bäuerle, Angew. Chem. Int. Ed. 2001, 40, 46774680 .

[47] K. Sivakumar, F. Xie, B. M. Cash, S. Long, H. N. Barnhill, Q. Wang, Org. Lett. 2004, 6, 4603-4606.

[48] N. Kitamura, T. Fukagawa, S. Kohtani, S.-i. Kitoh, K.-K. Kunimoto, R. Nakagaki, J. Photochem. Photobiol. A 2007, 188, 378-386.

[49] a) G. Chen, D. J. Yee, N. G. Gubernator, D. Sames, J. Am. Chem. Soc. 2005, 127, 4544-4545; b) G. Chen, N. Gubernator, D. Sames, D. J. Yee, 2006.

[50] T. Sheshashena Reddy, A. Ram Reddy, Dyes Pigm. 2013, 96, 525-534. 
[51] C. Murata, T. Masuda, Y. Kamochi, K. Todoroki, H. Yoshida, H. Nohta, M. Yamaguchi, A. Takadate, Chem. Pharm. Bull. 2005, 53, 750-758.

[52] K. Komatsu, Y. Urano, H. Kojima, T. Nagano, J. Am. Chem. Soc. 2007, 129, 13447-13454.

[53] B. N. Ahamed, P. Ghosh, Dalton Trans. 2011, 40, 6411-6419.

[54] a) Y. Peng, Y.-M. Dong, M. Dong, Y.-W. Wang, J. Org. Chem. 2012, 77, 90729080; b) C. Wang, S. Yang, M. Yi, C. Liu, Y. Wang, J. Li, Y. Li, R. Yang, ACS Appl. Mater. Interfaces 2014, 6, 9768-9775.

[55] T.-I. Kim, H. Kim, Y. Choi, Y. Kim, Chem. Comm. 2011, 47, 9825-9827.

[56] P. Hou, S. Chen, H. Wang, J. Wang, K. Voitchovsky, X. Song, Chem. Comm. 2014, 50, 320-322.

[57] a) M.-T. Le Bris, J. Mugnier, J. Bourson, B. Valeur, Chem. Phys. Lett. 1984, 106, 124-127; b) C. Trebaul, J. Roncali, F. Garnier, R. Guglielmetti, Bull. Chem. Soc. Jpn. 1987, 60, 2657-2662; c) S. Fery-Forgues, M. T. Le Bris, J. C. Mialocq, J. Pouget, W. Rettig, B. Valeur, J. Phys. Chem. 1992, 96, 701-710.

[58] M. Monsigny, P. Midoux, M.-T. Le Bris, A.-C. Roche, B. Valeur, Biol. Cell 1989, 67, 193-200.

[59] H. M. Kim, P. R. Yang, M. S. Seo, J.-S. Yi, J. H. Hong, S.-J. Jeon, Y.-G. Ko, K. J. Lee, B. R. Cho, J. Org. Chem. 2007, 72, 2088-2096.

[60] I. Kim, D. Kim, S. Sambasivan, K. H. Ahn, Asian J. Org. Chem. 2012, 1, 60-64.

[61] A. R. Sarkar, C. H. Heo, H. W. Lee, K. H. Park, Y. H. Suh, H. M. Kim, Anal. Chem. 2014, 86, 5638-5641.

[62] L. G. Lee, G. M. Berry, C.-H. Chen, Cytometry 1989, 10, 151-164.

[63] R. P. Haugland, J. Whitaker, US4945171 A.

[64] J. E. Whitaker, R. P. Haugland, F. G. Prendergast, Anal. Biochem. 1991, 194, 330-344.

[65] a) C. J. Chang, S. J. Lippard, E. Nolan, WO2005067580 A3; b) E. M. Nolan, S. J. Lippard, J. Am. Chem. Soc. 2007, 129, 5910-5918; c) A. Minta, P. R. Escamilla, WO2011047391 A1.

[66] M. D. Pluth, M. R. Chan, L. E. McQuade, S. J. Lippard, Inorg. Chem. 2011, 50, 9385-9392.

[67] R. M. Strongin, Y. Guo, L. Hakuna, M. A. Lowry, C. J. O. Escobedo, WO2013126816 A1.

[68] D. Srikun, A. E. Albers, C. J. Chang, Chem. Sci. 2011, 2, 1156-1165. 
[69] S. H. Kim, J. R. Gunther, J. A. Katzenellenbogen, Org. Lett. 2008, 10, 49314934.

[70] W. M. F. Fabian, S. Schuppler, O. S. Wolfbeis, J. Chem. Soc., Perkin Trans. 2 1996, 853-856.

[71] a) Y. Yang, M. Lowry, C. M. Schowalter, S. O. Fakayode, J. O. Escobedo, X. Xu, H. Zhang, T. J. Jensen, F. R. Fronczek, I. M. Warner, R. M. Strongin, J. Am. Chem. Soc. 2006, 128, 14081-14092; b) Y. Yang, M. Lowry, X. Xu, J. O. Escobedo, M. Sibrian-Vazquez, L. Wong, C. M. Schowalter, T. J. Jensen, F. R. Fronczek, I. M. Warner, R. M. Strongin, Proc. Natl. Acad. Sci. U.S.A. 2008, 105, 8829-8834.

[72] a) K. Furukawa, H. Abe, J. Wang, M. Uda, H. Koshino, S. Tsuneda, Y. Ito, Org. Biomol. Chem. 2009, 7, 671-677; b) M. Sibrian-Vazquez, J. O. Escobedo, M. Lowry, R. M. Strongin, Pure Appl. Chem. 2012, 84, 2443-2456.

[73] a) J. Chen, W. Liu, B. Zhou, G. Niu, H. Zhang, J. Wu, Y. Wang, W. Ju, P. Wang, J. Org. Chem. 2013, 78, 6121-6130; b) A. Chevalier, P.-Y. Renard, A. Romieu, Chem.-Eur. J. 2014, 20, 8330-8337.

[74] Z. Tian, B. Tian, J. Zhang, Dyes Pigm. 2013, 99, 1132-1136.

[75] L. Wu, K. Burgess, Org. Lett. 2008, 10, 1779-1782.

[76] A. Zilles, K. H. Drexhage, N. U. Kemnitzer, J. Arden-Jacob, M. HamersSchneider, US20130224871 A1.

[77] G. Ulrich, R. Ziessel, A. Harriman, Angew. Chem. Int. Ed. 2008, 47, 1184-1201.

[78] Y. Chen, J. Zhao, H. Guo, L. Xie, J. Org. Chem. 2012, 77, 2192-2206.

[79] a) A. Martin, C. Long, R. J. Forster, T. E. Keyes, Chem. Commun. 2012, 48, 5617-5619; b) A. Martin, R. D. Moriarty, C. Long, R. J. Forster, T. E. Keyes, Asian J. Org. Chem. 2013, 2, 763-778.

[80] M. Verdoes, K. Oresic Bender, E. Segal, W. A. van der Linden, S. Syed, N. P. Withana, L. E. Sanman, M. Bogyo, J. Am. Chem. Soc. 2013, 135, 14726-14730.

[81] A. Y. Bochkov, I. O. Akchurin, O. A. Dyachenko, V. F. Traven, Chem. Commun. 2013, 49, 11653-11655.

[82] Y. Zhou, Y. Xiao, S. Chi, X. Qian, Org. Lett. 2008, 10, 633-636.

[83] J. F. Araneda, W. E. Piers, B. Heyne, M. Parvez, R. McDonald, Angew. Chem. Int. Ed. 2011, 50, 12214-12217.

[84] M. Mao, S. Xiao, T. Yi, K. Zou, J. Fluorine Chem. 2011, 132, 612-616.

[85] Z. Li, X. Lv, Y. Chen, W.-F. Fu, Dyes Pigm. 2014, 105, 157-162. 
[86] a) X. Peng, F. Song, E. Lu, Y. Wang, W. Zhou, J. Fan, Y. Gao, J. Am. Chem. Soc. 2005, 127, 4170-4171; b) W. Pham, L. Cassell, A. Gillman, D. Koktysh, J. C. Gore, Chem. Commun. 2008, 1895-1897.

[87] F. Vollmer, W. Rettig, E. Birckner, J Fluoresc 1994, 4, 65-69.

[88] B. Valeur, in Molecular Fluorescence, Wiley-VCH Verlag GmbH, 2001, pp. 72124.

[89] B. Valeur, in Molecular Fluorescence, Wiley-VCH Verlag GmbH, 2001, pp. 226246.

[90] J. Kasurinen, Biochem. Biophys. Res. Commun. 1992, 187, 1594-1601.

[91] P. L. Paris, J. M. Langenhan, E. T. Kool, Nucleic Acids Res. 1998, 26, 3789-3793.

[92] a) E. V. Bichenkova, A. Sardarian, H. E. Savage, C. Rogert, K. T. Douglas, Assay Drug Dev. Technol. 2005, 3, 39-46; b) E. V. Bichenkova, H. E. Savage, A. R. Sardarian, K. T. Douglas, Biochem. Biophys. Res. Commun. 2005, 332, 956-964; c) E. V. Bichenkova, A. Gbaj, L. Walsh, H. E. Savage, C. Rogert, A. R. Sardarian, L. L. Etchells, K. T. Douglas, Org. Biomol. Chem. 2007, 5, 1039-1051.

[93] a) Y. N. Teo, E. T. Kool, Chem. Rev. 2012, 112, 4221-4245; b) H. Kashida, H. Asanuma, Phys. Chem. Chem. Phys. 2012, 14, 7196-7204.

[94] a) J. Gao, C. Strässler, D. Tahmassebi, E. T. Kool, J. Am. Chem. Soc. 2002, 124, 11590-11591; b) J. Gao, S. Watanabe, E. T. Kool, J. Am. Chem. Soc. 2004, 126, 12748-12749; c) A. Cuppoletti, Y. Cho, J.-S. Park, C. Strässler, E. T. Kool, Bioconjugate Chem. 2005, 16, 528-534; d) J. N. Wilson, E. T. Kool, Org. Biomol. Chem. 2006, 4, 4265-4274; e) Y. N. Teo, J. N. Wilson, E. T. Kool, Chem.-Eur. J. 2009, 15, 11551-11558; f) Y. N. Teo, E. T. Kool, Bioconjugate Chem. 2009, 20, 2371-2380.

[95] a) M. Nakamura, Y. Ohtoshi, K. Yamana, Chem. Commun. 2005, 5163-5165; b) M. Nakamura, Y. Shimomura, Y. Ohtoshi, K. Sasa, H. Hayashi, H. Nakano, K. Yamana, Org. Biomol. Chem. 2007, 5, 1945-1951; c) M. Nakamura, Y. Murakami, K. Sasa, H. Hayashi, K. Yamana, J. Am. Chem. Soc. 2008, 130, 69046905.

[96] C.-C. Hsieh, M.-L. Ho, P.-T. Chou, in Advanced Fluorescence Reporters in Chemistry and Biology I, Vol. 8 (Ed.: A. P. Demchenko), Springer, 2010, pp. 225266.

[97] J. Zhao, S. Ji, Y. Chen, H. Guo, P. Yang, Phys. Chem. Chem. Phys. 2012, 14, 8803-8817. 
[98] M. Van der Auweraer, Z. R. Grabowski, W. Rettig, J. Phys. Chem. 1991, 95, 2083-2092.

[99] V. A. Galievsky, S. I. Druzhinin, A. Demeter, Y.-B. Jiang, S. A. Kovalenko, L. Pérez Lustres, K. Venugopal, N. P. Ernsting, X. Allonas, M. Noltemeyer, R. Machinek, K. A. Zachariasse, ChemPhysChem 2005, 6, 2307-2323.

[100] K. Rotkiewicz, K. H. Grellmann, Z. R. Grabowski, Chem. Phys. Lett. 1973, 19, 315-318.

[101] Z. R. Grabowski, K. Rotkiewicz, A. Siemiarczuk, J. Lumin. 1979, 18-19, 420424.

[102] T. Taniguchi, J. Wang, S. Irle, S. Yamaguchi, Dalton Trans. 2013, 42, 620-624.

[103] J. Fan, M. Hu, P. Zhan, X. Peng, Chem. Soc. Rev. 2013, 42, $29-43$.

[104] B. Wieb van der Meer, in FRET - Förster Resonance Energy Transfer (Eds.: I. Medintz, N. Hildebrandt), Wiley-VCH, 2013, pp. 23-62.

[105] G.-S. Jiao, L. H. Thoresen, K. Burgess, J. Am. Chem. Soc. 2003, 125, 1466814669.

[106] L. Yuan, W. Lin, Z. Cao, J. Wang, B. Chen, Chem.-Eur. J. 2012, 18, 1247-1255.

[107] S. Speiser, Chem. Rev. 1996, 96, 1953-1976.

[108] W. Lin, L. Yuan, Z. Cao, Y. Feng, J. Song, Angew. Chem. Int. Ed. 2010, 49, 375379.

[109] D. Su, J. Oh, S.-C. Lee, J. M. Lim, S. Sahu, X. Yu, D. Kim, Y.-T. Chang, Chem. Sci. 2014, 5, 4812-4818.

[110] B. R. Rankin, R. R. Kellner, S. W. Hell, Opt. Lett. 2008, 33, 2491-2493.

[111] a) K. Kolmakov, V. N. Belov, J. Bierwagen, C. Ringemann, V. Müller, C. Eggeling, S. W. Hell, Chem.-Eur. J. 2010, 16, 158-166; b) C. Wurm, K. Kolmakov, F. Gottfert, H. Ta, M. Bossi, H. Schill, S. Berning, S. Jakobs, G. Donnert, V. Belov, S. Hell, Opt. Nanoscopy 2012, 1, 7; c) K. Kolmakov, C. A. Wurm, D. N. H. Meineke, F. Göttfert, V. P. Boyarskiy, V. N. Belov, S. W. Hell, Chem.-Eur. J. 2014, 20, 146-157.

[112] Y. F. Hua, R. Sinha, C. S. Thiel, R. Schmidt, J. Huve, H. Martens, S. W. Hell, A. Egner, J. Klingauf, Nat. Neurosci. 2011, 14, 833-839.

[113] K. Friedemann, A. Turshatov, K. Landfester, D. Crespy, Langmuir 2011, 27, $7132-7139$.

[114] P. A. Pellett, X. Sun, T. J. Gould, J. E. Rothman, M.-Q. Xu, I. R. Corrêa, J. Bewersdorf, Biomed. Opt. Express 2011, 2, 2364-2371. 
[115] C. Dean, H. Liu, T. Staudt, M. A. Stahlberg, S. Vingill, J. Bückers, D. Kamin, J. Engelhardt, M. B. Jackson, S. W. Hell, E. R. Chapman, J. Neurosci. 2012, 32, 5398-5413.

[116] T. Karlsson, M. V. Turkina, O. Yakymenko, K.-E. Magnusson, E. Vikström, PLoS Pathog. 2012, 8, e1002953.

[117] E. M. Mace, J. S. Orange, Commun. Integr. Biol. 2012, 5, 184-186.

[118] C. Kempf, T. Staudt, P. Bingen, H. Horstmann, J. Engelhardt, S. W. Hell, T. Kuner, PLoS ONE 2013, 8, e62893.

[119] G. Lavieu, M. H. Dunlop, A. Lerich, H. Zheng, F. Bottanelli, J. E. Rothman, Mol. Biol. Cell 2014, 25, 3028-3036.

[120] B. Henkel, D. R. Drose, T. Ackels, S. Oberland, M. Spehr, E. M. Neuhaus, Chem. Senses 2014, 40, 73-87.

[121] L. Westin, M. Reuss, M. Lindskog, A. Aperia, H. Brismar, BMC Neurosci. 2014, $15,45$.

[122] F. Wilfling, A. R. Thiam, M.-J. Olarte, J. Wang, R. Beck, T. J. Gould, E. S. Allgeyer, F. Pincet, J. Bewersdorf, R. V. Farese, T. C. Walther, eLife 2014, 3, e01607.

[123] A. Johnson, N. Bhattacharya, M. Hanna, J. G. Pennington, A. L. Schuh, L. Wang, M. S. Otegui, S. M. Stagg, A. Audhya, The EMBO journal 2015, embj.201489032.

[124] W. H. Perkin, J. Chem. Soc. 1868, 21, 53-63.

[125] P. A. Vadola, D. Sames, J. Org. Chem. 2012, 77, 7804-7814.

[126] W. D. Kumler, J. J. Eiler, J. Am. Chem. Soc. 1943, 65, 2355-2361.

[127] L. D. Freedman, G. O. Doak, Chem. Rev. 1957, 57, 479-523.

[128] C. Hansch, A. Leo, R. W. Taft, Chem. Rev. 1991, 91, 165-195.

[129] M.-E. Theoclitou, L. A. Robinson, Tetrahedron Lett. 2002, 43, 3907-3910.

[130] J. L. Luche, J. Am. Chem. Soc. 1978, 100, 2226-2227.

[131] A. R. Katritzky, C. A. Ramsden, J. A. Joule, V. V. Zhdankin, in Handbook of Heterocyclic Chemistry (Third Edition) (Eds.: A. R. Katritzky, C. A. Ramsden, J. A. Joule, V. V. Zhdankin), Elsevier, 2010, pp. 37-86.

[132] A. R. Katritzky, C. A. Ramsden, J. A. Joule, V. V. Zhdankin, in Handbook of Heterocyclic Chemistry (Third Edition) (Eds.: A. R. Katritzky, C. A. Ramsden, J. A. Joule, V. V. Zhdankin), Elsevier, 2010, pp. 242-382.

[133] J. W. Perich, R. B. Johns, Synthesis 1988, 1988, 142-144. 
[134] O. Valdes-Aguilera, D. C. Neckers, Acc. Chem. Res. 1989, 22, 171-177.

[135] The Molecular Probes Handbook: A Guide to Fluorescent Probes and Labeling Technologies, $11^{\text {th }}$ ed., Life Technologies, 2010.

[136] M. E. Kuipers, P. J. Swart, M. M. W. B. Hendriks, D. K. F. Meijer, J. Med. Chem. 1995, 38, 883-889.

[137] G. Moneron, R. Medda, B. Hein, A. Giske, V. Westphal, S. W. Hell, Opt. Express 2010, 18, 1302-1309.

[138] J. H. Blanch, J. Chem. Soc. B 1966, 937-939.

[139] R.-Z. Ma, Q.-C. Yao, X. Yang, M. Xia, J. Fluorine Chem. 2012, 137, 93-98.

[140] R. Bentley, T. S. Stevens, M. Thompson, J. Chem. Soc. C 1970, 791-795.

[141] J. R. Lakowicz, in Principles of Fluorescence Spectroscopy (Ed.: J. R. Lakowicz), Springer, 2006, pp. 331-351.

[142] a) L. Kaczmarek, R. Balicki, J. Lipkowski, P. Borowicz, A. Grabowska, J. Chem. Soc., Perkin Trans. 2 1994, 1603-1610; b) D. LeGourriérec, V. Kharlanov, R. G. Brown, W. Rettig, J. Photochem. Photobiol., A 1998, 117, 209-216; c) N. Basaric, P. Wan, Photochem. Photobiol. Sci. 2006, 5, 656-664.

[143] C. Eggeling, J. Widengren, R. Rigler, C. A. M. Seidel, in Applied Fluorescence in Chemistry, Biology and Medicine, Springer, 1999, pp. 193-240.

[144] K. Muthuramu, V. R. Murthy, J. Org. Chem. 1982, 47, 3976-3979.

[145] a) B. H. Winters, H. I. Mandelberg, W. B. Mohr, Appl. Phys. Lett. 1974, 25, 723725; b) R. J. von Trebra, T. H. Koch, J. Photochem. 1986, 35, 33-46.

[146] O. V. Zinkovskaya, N. A. Kuznetsova, O. L. Kaliya, J. Appl. Spectrosc. 1984, 41, 1171-1174.

[147] M. Krzeszewski, O. Vakuliuk, D. T. Gryko, Eur. J. Org. Chem. 2013, 2013, 5631-5644.

[148] a) T. R. Kelly, M. H. Kim, J. Org. Chem. 1992, 57, 1593-1597; b) G.-H. Kuo, A. Wang, S. Emanuel, A. DeAngelis, R. Zhang, P. J. Connolly, W. V. Murray, R. H. Gruninger, J. Sechler, A. Fuentes-Pesquera, D. Johnson, S. A. Middleton, L. Jolliffe, X. Chen, J. Med. Chem. 2004, 48, 1886-1900.

[149] T. Bakos, I. Vincze, Synth. Commun. 1989, 19, 523-528.

[150] A. N. Shaw, K. J. Duffy, R. Tedesco, K. Wiggall, WO2007136990 A2.

[151] J.-H. Lee, W.-S. Kim, Y. Y. Lee, C.-G. Cho, Tetrahedron Lett. 2002, 43, 57795782 .

[152] X. Han, B. M. Stoltz, E. J. Corey, J. Am. Chem. Soc. 1999, 121, 7600-7605. 
[153] R. L. Atkins, D. E. Bliss, J. Org. Chem. 1978, 43, 1975-1980.

[154] H. Takechi, S. Kamada, M. Machida, Chem. Pharm. Bull. 1996, 44, 793-799.

[155] J. E. Whitaker, R. P. Haugland, D. Ryan, P. C. Hewitt, R. P. Haugland, F. G. Prendergast, Anal. Biochem. 1992, 207, 267-279.

[156] a) J. Arden-Jacob, J. Frantzeskos, N. U. Kemnitzer, A. Zilles, K. H. Drexhage, Spectrochim. Acta, Part A 2001, 57, 2271-2283; b) P. V. Fisher, R. O'neill, WO2004003510 A3; c) J. Arden-Jacob, K. H. Drexhage, J. Frantzeskos, A. Zilles, EP1173519 B1; d) K. Kolmakov, V. N. Belov, C. A. Wurm, B. Harke, M. Leutenegger, C. Eggeling, S. W. Hell, Eur. J. Org. Chem. 2010, 2010, 35933610.

[157] K. Kolmakov, C. Wurm, M. V. Sednev, M. L. Bossi, V. N. Belov, S. W. Hell, Photochem. Photobiol. Sci. 2012, 11, 522-532.

[158] J. B. Grimm, A. J. Sung, W. R. Legant, P. Hulamm, S. M. Matlosz, E. Betzig, L. D. Lavis, ACS Chem. Biol. 2013, 8, 1303-1310.

[159] G. W. Gribble, C. F. Nutaitis, Synthesis 1987, 1987, 709-711.

[160] H. Mansilla, D. Regás, Synth. Commun. 2006, 36, 2195-2201.

[161] A. Thurkauf, A. E. Jacobson, K. C. Riee, Synthesis 1988, 1988, 233-234.

[162] L. Alig, J. Alsenz, M. Andjelkovic, S. Bendels, A. Bénardeau, K. Bleicher, A. Bourson, P. David-Pierson, W. Guba, S. Hildbrand, D. Kube, T. Lübbers, A. V. Mayweg, R. Narquizian, W. Neidhart, M. Nettekoven, J.-M. Plancher, C. Rocha, M. Rogers-Evans, S. Röver, G. Schneider, S. Taylor, P. Waldmeier, J. Med. Chem. 2008, 51, 2115-2127.

[163] W. P. Neumann, W. Uzick, A. K. Zarkadis, J. Am. Chem. Soc. 1986, 108, 37623770 .

[164] A. Ono, N. Suzuki, J. Kamimura, Synthesis 1987, 1987, 736-738.

[165] L. Gu, B. Yang, F. Liu, Y. Bai, Chin. J. Chem. 2009, 27, 1199-1201.

[166] R. W. A. Luke, P. G. T. Boyce, E. Kate Dorling, Tetrahedron Lett. 1996, 37, 263266.

[167] W.-C. Sun, K. R. Gee, D. H. Klaubert, R. P. Haugland, J. Org. Chem. 1997, 62, 6469-6475.

[168] a) J. Burdon, B. L. Kane, J. C. Tatlow, J. Fluorine Chem. 1971, 1, 185-192; b) A. M. Kenwright, G. Sandford, A. J. Tadeusiak, D. S. Yufit, J. A. K. Howard, P. Kilickiran, G. Nelles, Tetrahedron 2010, 66, 9819-9827.

[169] S. Nahm, S. M. Weinreb, Tetrahedron Lett. 1981, 22, 3815-3818. 
[170] D. N. Kursanov, Z. N. Parnes, N. M. Loim, Synthesis 1974, 1974, 633-651.

[171] K. Kolmakov, C. A. Wurm, R. Hennig, E. Rapp, S. Jakobs, V. N. Belov, S. W. Hell, Chem.-Eur. J. 2012, 18, 12986-12998.

[172] a) M. M. Martin, L. Lindqvist, J. Lumin. 1975, 10, 381-390; b) E. Martin, A. Pardo, M. S. Guijarro, J. I. Fernandez-Alonso, J. Mol. Struct. 1986, 142, 197-200;

c) R. Sjöback, J. Nygren, M. Kubista, Spectrochim. Acta, Part A 1995, 51, L7L21; d) N. Klonis, W. Sawyer, J. Fluoresc. 1996, 6, 147-157.

[173] T. Peng, D. Yang, Org. Lett. 2010, 12, 496-499.

[174] J. Bückers, D. Wildanger, G. Vicidomini, L. Kastrup, S. W. Hell, Opt. Express 2011, 19, 3130-3143.

[175] N. Senda, A. Momotake, Y. Nishimura, T. Arai, Bull. Chem. Soc. Jpn. 2006, 79, 1753-1757.

[176] R. K. Olsen, J. Org. Chem. 1970, 35, 1912-1915.

[177] K.-J. Han, M. Kim, Lett. Org. Chem. 2004, 4, 20-22. 


\section{Curriculum vitae}

Name: $\quad$ Maksim Sednev

Date of birth: $\quad 21.10 .1988$

Place of birth: Nizhny Tagil

Nationality: $\quad$ Russian

EDUCATION

2011 - pre- University of Göttingen and Max Planck Institute for Biophysical sent Chemistry, Germany

$P h D$ in Organic Chemistry

Thesis: Fluorescent dyes with large Stokes shifts of $80-200 \mathrm{~nm}$ and their use in super-resolution microscopy

Supervisors: Prof. Dr. Stefan W. Hell, Prof. Dr. Armin de Meijere

2011

Max Planck Institute for Biophysical Chemistry, Germany

Visiting student

Project: Masked red-emitting carbopyronines with photosensitive 2diazo-1-indanone caging group

Supervisor: Prof. Dr. Stefan W. Hell

2005 - 2010 Saint Petersburg State University, Russia

MSc with Distinction in Chemistry

Thesis: 1,3-Dipolar cycloaddition of (Z)-1-arylmethylidene-3-oxopyrazolidine-1-ium-2-ide to $N$-arylmaleimides

Supervisor: Assoc. Prof. Yuri B. Koptelov

\section{AWARDS AND FELLOWSHIPS}

Microsoft Fellowship for the participation in the $63^{\text {rd }}$ Lindau Nobel Laureate Meeting, Lindau, Germany 
2011 - 2014 Max Planck Society Graduate Fellowship

\section{PUBLICATIONS}

6. submitted: Sednev, M.V.; Belov, V.N.; Hell, S.W: Fluorescent dyes with large Stokes shifts for super-resolution optical microscopy of biological objects. Methods Appl. Fluoresc., 2015

5. Sednev, M.V.; Wurm, C.A.; Belov, V.N.; Hell, S.W: Carborhodol: A New Hybrid Fluorophore Obtained by Combination of Fluorescein and Carbopyronine Dye Cores. Bioconjugate Chem. 24 (4), 690-700, 2013

4. Nizamov, S; Willig, K.I; Sednev, M.V.; Belov, V.N.; Hell, S.W.: Phosphorylated 3Heteroarylcoumarins and Their Use in Fluorescence Microscopy and Nanoscopy. Chem. Eur. J. 18 (51), 16339-16348, 2012

3. Kolmakov, K.; Wurm, C.; Sednev, M. V.; Bossi, M. L.; Belov, V.N.; Hell, S. W.: Masked Red-emitting Carbopyronine Dyes with Photosensitive 2-Diazo-1-indanone Caging Group. Photochem. Photobiol. Sci. 11 (3), 522-532, 2012

2. Koptelov, Yu. B.; Sednev, M.V.; Kostikov, R.R.: (Z)-1-Alkylidene- and 1Arylmethylidene-5,5-dimethyl-3-oxopyrazolidin-1-ium-2-ides and Their Cycloaddition to N-Arylmaleimides. Russ. J. Org. Chem. 48 (6), 804-814, 2012

1. Koptelov, Yu. B.; Sednev, M.V.: Stable Azomethine Imines Derived from Pyrazolidin-3-one and Their Cycloaddition to N-Arylmaleimides. Russ. J. Org. Chem. 47 (3), 547-555, 2011

\section{CONFERENCES AND MEETINGS}

2014 Labeling and Nanoscopy, Heidelberg, Germany “Design of Red-Emitting Dyes for Microscopy and Nanoscopy” (poster)

$2013 \quad 1^{\text {th }}$ JCF-Frühjahrsymposium (A meeting of young chemists organized by German Chemical Society), Berlin, Germany "(Iso)quinolinocoumarins: a novel class of bright fluorescent dyes with large Stokes shift” (talk and poster)

$13^{\text {th }}$ Belgian Organic Synthesis Symposium, Leuven, Belgium "Novel coumarin dyes for microscopy applications" (poster)

V International Symposium “Aliphatic Diazo Compounds, St. Petersburg, Russia

"Cycloaddition of $\mathrm{N}$-arylmaleimides to $\mathrm{C}$-alkyl- and $\mathrm{C}$-arylsubstituted azomethine imines generated from 5,5-dimethylpyrazolidin-3-one" (poster) 
TEACHING EXPERIENCE

2013 Teaching assistant, Introductory Organic Synthesis Lab, University of Göttingen

2012 Teaching assistant, Introductory Organic Synthesis Lab, University of Göttingen

2011 - 2012 Teaching assistant, Advanced Organic Synthesis Lab, University of Göttingen

Date:

Signature: 